\title{
A premouse inheriting strong cardinals from $V$
}

\author{
Farmer Schlutzenberg ${ }^{1}$
}

\begin{abstract}
We identify a premouse inner model $L[\mathbb{E}]$, such that for any coarsely iterable background universe $R$ modelling ZFC, $L[\mathbb{E}]^{R}$ is a proper class premouse of $R$ inheriting all strong and Woodin cardinals from $R$. Moreover, for each $\alpha \in \mathrm{OR}$, $L[\mathbb{E}]^{R} \mid \alpha$ is $(\omega, \alpha)$-iterable, via iteration trees which lift to coarse iteration trees on $R$.

We prove that $(k+1)$-condensation follows from $(k+1)$-solidity together with $\left(k, \omega_{1}+1\right)$-iterability (that is, roughly, iterability with respect to normal trees). We also prove that a slight weakening of $(k+1)$-condensation follows from $\left(k, \omega_{1}+1\right)$-iterability (without the $(k+1)$-solidity hypothesis).

The results depend on the theory of generalizations of bicephali, which we also develop.

Keywords: bicephalus, condensation, normal iterability, inner model, strong cardinal

2010 MSC: 03E45, 03E55
\end{abstract}

\section{Introduction}

Consider fully iterable, sound premice $M, N$ with $M|\rho=N| \rho$ and $\rho_{\omega}^{M}=\rho=$ $\rho_{\omega}^{N}$. Under what circumstances can we deduce that either $M \unlhd N$ or $N \unlhd M$ ? This conclusion follows if $\rho$ is a cutpoint of both models. By [2, Lemma 3.1], ${ }^{1}$ the conclusion also follows if $\rho$ is a regular uncountable cardinal in $V$ and there is no premouse with a superstrong extender. We will show that if $M\left\|\rho^{+M}=N\right\| \rho^{+N}$ and $M, N$ have a certain joint iterability property, then $M=N$.

The joint iterability property and the proof that $M=N$, is motivated by the bicephalus argument of [3, §9]. Bicephali in [3] are structures $B=(P, E, F)$, where both $(P, E)$ and $(P, F)$ are active premice. If $B$ is an iterable bicephalus and there is no iterable superstrong premouse then $E=F$ (see $[3, \S 9]$ and [12]); the proof is by comparison of $B$ with itself. In $\S 3$ we consider a more general form of bicephali, including, for example, the structure $C=(\rho, M, N)$, where $\rho, M, N$ are as at the end of the previous paragraph. If $C$ is iterable, a comparison of $C$ with itself will be used to show that $M=N$ in this situation.

\footnotetext{
Email address: farmer.schlutzenberg@gmail.com (Farmer Schlutzenberg)

${ }^{1}$ The paper [2] literally deals with premice with Jensen indexing, whereas we deal with Mitchell-Steel indexing. However, the same result still holds.
} 
Hugh Woodin also noticed that generalizations of bicephali can be used in certain fine structural arguments, probably before the author did; see [17]. The bicephali used in [17] have more closure than those considered here, but of course, the premice of [17] are long extender premice. So while there is some overlap, it seems that things are quite different.

We will also consider bicephali $\left(\rho^{\prime}, M^{\prime}, N^{\prime}\right)$ in which $M^{\prime}$ or $N^{\prime}$ might fail to be fully sound. However, we will assume that both $M^{\prime}, N^{\prime}$ project $\leq \rho^{\prime}$, are $\rho^{\prime}$-sound, and $M^{\prime}, N^{\prime}$ agree below their common value for $\left(\rho^{\prime}\right)^{+}$. If such a bicehpalus is iterable, it might be that $M^{\prime} \neq N^{\prime}$, but we will see that in this situation, $M^{\prime}$ is an ultrapower of some premouse by an extender in the extender sequence $\mathbb{E}_{+}^{N^{\prime}}$ of $N^{\prime}$ (here $\mathbb{E}_{+}^{N^{\prime}}$ includes the active extender of $N^{\prime}$ ) or vice versa.

We will also prove similar results regarding cephalanxes, a blend of bicephali and phalanxes. The presence of superstrong premice makes cephalanxes somewhat more subtle than bicephali.

We give two applications of these results. First, in $\S 5$, we consider proving condensation from normal iterability. Let $k<\omega$, let $H, M$ be $k$-sound premice, $\pi: H \rightarrow M$ be a near $k$-embedding ${ }^{2}, \rho_{k+1}^{H} \leq \rho<\rho_{k}^{H}$, and suppose $H$ is $\rho$-sound and $\rho \leq \operatorname{cr}(\pi)$. We wish to prove the conclusion of $(k+1)$-condensation for this embedding. ${ }^{3}$ The classical (phalanx-based) proof of condensation uses the $\left(k, \omega_{1}, \omega_{1}+1\right)^{*}$-iterability of $M$ (roughly, iterability for stacks of normal trees), through its appeal to weak Dodd-Jensen. We would like to reduce this assumption to $\left(k, \omega_{1}+1\right)$-iterability (roughly, iterability for normal trees). Given the latter, and also assuming $M$ is $(k+1)$-solid, we will deduce the usual conclusion of condensation. We will also prove a slight weakening of $(k+1)$-condensation from $\left(k, \omega_{1}+1\right)$-iterability, without the extra solidity hypothesis. (As we are not assuming $\left(k, \omega_{1}, \omega_{1}+1\right)^{*}$-iterability, it is natural to consider the circumstance that $M$ fails to be $(k+1)$-solid; see $\S 7 .^{4}$ But note that the assumption that $H$ is $\rho$-sound entails that $\left(H, p_{k+1}^{H} \backslash \rho\right)$ is $(k+1)$-solid.) Our proof makes substitutes bicephali and cephalanxes for phalanxes, and avoids (weak) Dodd-Jensen. ${ }^{56}$

Let $W \models$ ZFC be coarsely iterable. Let $N$ be the output of a (standard) fully backgrounded $L[\mathbb{E}]$-construction of $W$. Then $N$ inherits the Woodin cardinals

\footnotetext{
${ }^{2}$ Actually we will work with the more general class of $k$-lifting embeddings; see 2.1.

${ }^{3}$ Approximately, that is, the "version ... with $\rho_{k+1}^{H}$ replacing $\rho_{\omega}^{H}$ " in [3, pp. 87-88], or [2, Lemma 1.3], though this uses Jensen indexing, or [18, Theorem 9.3.2], though this uses Jensen indexing and $\Sigma^{*}$-fine structure.

4 Actually, the author has since shown that $(k+1)$-solidity follows from $\left(k, \omega_{1}+1\right)$ iterability. This result will appear in [7]. So the present paper together with [7] gives a complete proof of $(k+1)$-condensation from $\left(k, \omega_{1}+1\right)$-iterability.

${ }^{5}$ The way we have presented our proof, we do make use of the standard proof of condensation, in proving 2.13, but in circumstances in which Dodd-Jensen is not required. This appeal to the standard proof can, however, be removed, by arranging things more inductively and using the main structure of the proof of 5.2 to prove 2.13 .

${ }^{6}$ Some of the key arguments involved here, and extensions thereof regarding solidity and universality, were presented by the author at the 3rd Münster conference on inner model theory, the core model induction, and hod mice, in July 2015. Some notes of those talks, taken by Schindler, can be seen in [6].
} 
of $W$, meaning that every Woodin cardinal of $W$ is Woodin in $N$. However, $\kappa$ can be strong in $W$, but not strong in $N$. For example, if $\kappa$ is strong in $W$ but $W$ has no measurable cardinal $\mu>\kappa$, then $N$ has no measurable cardinal $\geq \kappa$ (see 6.1); in particular, $\kappa$ is not even measurable in $N$, let alone strong.

In [15], assuming that $W$ is a (finely) iterable premouse with no largest cardinal, Steel defined the local $K^{c}$-construction $K_{\mathrm{loc}}^{W}$ of $W$, such that $K_{\mathrm{loc}}^{W}$ inherits both Woodin and strong cardinals from $W$. Along with requiring that $W$ be a premouse, an important feature used in ensuring that strong cardinals are inherited is that the background extenders used to construct $K_{\text {loc }}^{W}$ do not have to be $W$-total. As a consequence, when one lifts iteration trees on $M$ to iteration trees $\mathcal{U}$ on $V$, the tree $\mathcal{U}$ might have drops.

In $\S 6$, working with background theory ZF, given any transitive class $W \models$ ZFC which is (sufficiently) coarsely iterable, we identify a new form of $L[\mathbb{E}]$ construction $\mathbb{C}$ of $W$. Letting $L[\mathbb{E}]$ be the final model of $\mathbb{C}$ (as computed in $W$ ), we show that (a) $L[\mathbb{E}]$ is a proper class premouse of $W$, outright definable over $W$, (b) if $\kappa$ is strong (Woodin) in $W$, then $\kappa$ is strong (Woodin) in $L[\mathbb{E}]$, as witnessed by $\mathbb{E}$, and (c) noting that $W$ might be proper class, if there is a (class) function $f: \mathrm{OR}^{W} \rightarrow W$ such that $f(\alpha)$ wellorders $V_{\alpha}^{W}$ for each $\alpha \in \mathrm{OR}^{W}$, then $L[\mathbb{E}]$ is iterable, with iteration trees on $L[\mathbb{E}]$ lifting to (coarse, hence nondropping) trees on $W$. Thus, we achieve many of the properties of the the local $K^{c}$-construction, but with the advantages that $W$ need not be a premouse, and (even if $W$ is a premouse) trees $\mathcal{U}$ on $W$ resulting from lifting trees on $L[\mathbb{E}]$ are coarse (and hence non-dropping). ${ }^{7}$

We finally remark that Steel's local $K^{c}$-construction seems to be more local than $\mathbb{C}$, and hence as one extra feature that it seems $\mathbb{C}$ might not: $K_{\text {loc }}^{W}$ also inherits all $\lambda$-strong cardinals of $W$, whenever $\lambda$ is a limit cardinal of $W$.

\subsection{Notation and terminology}

\subsubsection{General}

The universe $N$ of a first-order structure $M=(N, \ldots)$ is denoted $\lfloor M\rfloor$.

We use the lexicographic order on [OR] $]^{<\omega}: a<b$ iff $a \neq b$ and $\max (a \Delta b) \in b$. We sometimes identify elements of $[\mathrm{OR}]^{<\omega}$ with strictly descending sequences of ordinals. Let $a \in[\mathrm{OR}]^{<\omega}$ with $a=\left\{a_{0}, \ldots, a_{k-1}\right\}$ where $a_{i}>a_{i+1}$ for all $i+1<k$. We write $a\left\lceil j\right.$ for $\left\{a_{0}, \ldots, a_{j-1}\right\}$.

\subsubsection{Premice}

We deal with premice and related structures with Mitchell-Steel indexing, but with extenders of superstrong type permitted on their extender sequence. That is, a super-fine extender sequence $\vec{E}$ is a sequence such that for each $\alpha \in \operatorname{dom}(\vec{E}), \vec{E}$ is acceptable at $\alpha$, and if $E_{\alpha} \neq \emptyset$ then either:

- $E_{\alpha}$ is a $(\kappa, \alpha)$ pre-extender over $\mathcal{J}_{\alpha}^{\vec{E}}$ and $E_{\alpha}$ is the trivial completion of $E_{\alpha}\left\lceil\nu\left(E_{\alpha}\right)\right.$ and $E_{\alpha}$ is not type Z, or

\footnotetext{
${ }^{7}$ The key ideas of the construction were presented by the author at the MAMLS 2014 meeting at Miami University.
} 
- $\mathcal{J}_{\alpha}^{\vec{E}}$ has largest cardinal $\nu$ and $E_{\alpha}$ is a $(\kappa, \nu)$ pre-extender over $\mathcal{J}_{\alpha}^{\vec{E}}$ and $i_{E_{\alpha}}(\kappa)=\nu=\nu\left(E_{\alpha}\right)$,

and further, properties 2 and 3 of [16, Definition 2.4] hold. We then define (potential) premice in terms of super-fine extender sequences, in the usual manner, with the caveat that we consider a (potential) premouse to be an amenable structure $P=\left(\mathcal{J}_{\alpha}^{\mathbb{E}}, \mathbb{E}, \widetilde{F}\right)$, where $\widetilde{F}$ is the amenable coding of the active extender $F$ of $P$, as described in [16, 2.9-2.10]. We may blur the distinction between $F$ and $\widetilde{F}$. Likewise for related terms, such as segmented-premouse (see $[12, \S 5]$ ). See $[8]$ for discussion of the modifications of the general theory needed to deal with these changes. ${ }^{8}$ We sometimes abbreviate premouse with pm and segmented-premouse with seg-pm. A premouse extender is the active extender of some premouse. ISC abbreviates "initial segment condition".

Let $P$ be a seg-pm. We write $F^{P}=F(P)$ for the active extender of $P$ (possibly $F^{P}=\emptyset$ ), $\mathbb{E}^{P}=\mathbb{E}(P)$ for the extender sequence of $P$, excluding $F^{P}$, and $\mathbb{E}_{+}^{P}=\mathbb{E}_{+}(P)=\mathbb{E}^{P \wedge} F^{P}$. If $F^{P} \neq \emptyset$ we write $\operatorname{lh}\left(F^{P}\right)=\mathrm{OR}^{P}$. We write $Q \unlhd P$ iff $Q$ is an initial segment of $P$ (that is, $\mathrm{OR}^{Q} \leq \mathrm{OR}^{P}$ and $\mathbb{E}_{+}^{Q}=\left(\mathbb{E}_{+}^{P}\right) \uparrow$ $\left.\left(\mathrm{OR}^{Q}+1\right)\right)$, and $Q \triangleleft P$ iff $Q \unlhd P$ but $Q \neq P$. Given a limit $\alpha \leq \mathrm{OR}^{P}$, we write $P \mid \alpha$ for the $Q \unlhd P$ such that $\mathrm{OR}^{Q}=\alpha$, and $P \| \alpha$ for its passivization $\left(\lfloor Q\rfloor, \mathbb{E}^{Q}, \emptyset\right)$. (So $P \| \alpha$ is passive, and $P \mid \alpha$ is active iff $\left(\mathbb{E}_{+}^{P}\right)_{\alpha} \neq \emptyset$.) If $P$ has a largest cardinal $\delta, \operatorname{lgcd}(P)$ denotes $\delta$. If $P$ is active, then $\nu(P)=\nu\left(F^{P}\right)$ denote the natural length of $F^{P}$, and $\iota(P)=\iota\left(F^{P}\right)$ denote $\max \left(\operatorname{lgcd}(P), \nu\left(F^{P}\right)\right)$. So if $P$ is an active premouse then $\iota(P)=\nu\left(F^{P}\right)$. Given also another seg-pm $R$ and an ordinal $\alpha \leq \min \left(\mathrm{OR}^{P}, \mathrm{OR}^{R}\right)$, we write $(P \sim R) \mid \alpha$ iff $P|\alpha=R| \alpha$. We also use such notation with more than two structures, and also with "||" replacing "|". We use similar notation for cephals; see 3.5.

Let $P$ be an active seg-pm, $F=F^{P}$ and $i_{F}^{P}: P \rightarrow \operatorname{Ult}(P, F)$ the ultrapower map. We say that $F$, or $P$, has superstrong type (or just is superstrong) iff $i_{F}^{P}(\operatorname{cr}(F))<\operatorname{lh}(F)$. (So if $F$ has superstrong type then $i_{F}^{P}(\operatorname{cr}(F)$ ) is the largest cardinal of $P$, and then $P$ is a premouse iff the initial segment condition holds for $P$.) In [12], all premice are assumed to be below superstrong type, but certain results there (in particular, $[12,2.17,2.20]$ ) hold in our context, by the same proofs, and when we cite these results, we literally refer to these generalizations. This generalization will be covered more explicitly in [7]. (However, the proof of [12, Theorem 5.3] does not go through at the superstrong level; Theorem 4.3 here generalizes that result at the superstrong level. $)^{9}$

${ }^{8}$ The only significant difference in the basic definitions (other than super-fine extender sequence) is that for $k$-maximal iteration tree $\mathcal{T}$, one must replace the usual requirement that $\operatorname{lh}\left(E_{\alpha}^{\mathcal{T}}\right)<\operatorname{lh}\left(E_{\beta}^{\mathcal{T}}\right)$ for all $\alpha<\beta$, with the requirement that $\operatorname{lh}\left(E_{\alpha}^{\mathcal{T}}\right) \leq \operatorname{lh}\left(E_{\beta}^{\mathcal{T}}\right)$ for all $\alpha<\beta$. However, we then get that $\operatorname{lh}\left(E_{\alpha}^{\mathcal{T}}\right)=\operatorname{lh}\left(E_{\beta}^{\mathcal{T}}\right)$ iff $\alpha+1=\beta$ and $E_{\alpha}^{\mathcal{T}}$ is superstrong and $M_{\alpha+1}^{* \mathcal{T}}$ is active type 2 with largest cardinal $\operatorname{cr}\left(E_{\alpha}^{\mathcal{T}}\right)$; in this case $M_{\alpha+1}^{\mathcal{T}}$ is active type 2 with ordinal height $\operatorname{lh}\left(E_{\alpha}^{\mathcal{T}}\right)$, and so $F\left(M_{\alpha+1}^{\mathcal{T}}\right)$ is the only possibility for $E_{\alpha+1}^{\mathcal{T}}$.

${ }^{9}$ The proof of Dodd-solidity for 1-sound, $\left(0, \omega_{1}, \omega_{1}+1\right){ }^{*}$-iterable premice (for Mitchell-Steel indexing) does not immediately generalize, although it can be adapted to the superstrong level with some further work; recall that Zeman [19] proves the analogous result for Jensen 


\subsubsection{Fine structure}

Let $M$ be a premouse. As in [16], $\mathfrak{C}_{0}(M)$ denotes the squash $M^{\text {sq }}$ of $M$ if $M$ is type 3 , and otherwise denotes $M$ (which is by definition amenable). If $M$ is non-type 3 , we also define $M^{\text {sq }}=M$, so in all cases, $\mathfrak{C}_{0}(M)=M^{\text {sq }}$. Also in general, $\mathfrak{C}_{0}(M)^{\text {unsq }}$ denotes $M$. We will often blur the distinction between $M$ and $\mathfrak{C}_{0}(M)$.

The (fine structural) pm language $\mathcal{L}$ is $\left\{\dot{\epsilon}, \doteq, \dot{\mathbb{E}}, \dot{\widetilde{F}}, \dot{F}_{\downarrow}\right\}$. We interpret $\mathcal{L}$ over $M$ (for seg-pms $M$ ) and over $\mathfrak{C}_{0}(M)$ (for premice $M$ ) in the usual manner. Over $M: \dot{\mathbb{E}}^{M}=\mathbb{E}^{M}, \dot{\widetilde{F}}^{M}=\widetilde{F}^{M}$, if $M$ is type 2 then $\dot{F}_{\downarrow}^{M}$ is the trivial completion of the largest non-type $\mathrm{Z}$ initial segment of $F^{M}$, and otherwise $\dot{F}_{\downarrow}^{M}=\emptyset$. Over $\mathfrak{C}_{0}(M)$ : as above if $M=\mathfrak{C}_{0}(M)$ is non-type 3 , so suppose $M$ is type 3 . Then $\dot{\mathbb{E}}^{\mathfrak{C}_{0}(M)}=\mathbb{E}^{\mathfrak{C}_{0}(M)}=\mathbb{E}^{M}\left\lceil\nu\left(F^{M}\right)\right.$, and $\dot{\widetilde{F}}^{\mathfrak{C}_{0}(M)}$ is the set of all restrictions $F^{M}\lceil\alpha$ for $\alpha<\nu\left(F^{M}\right)$, and $\dot{F}_{\downarrow}^{\mathfrak{C}_{0}(M)}=\emptyset$.

The language for the definability classes $\mathrm{r} \Sigma_{0}^{M}$ and $\mathrm{r} \Sigma_{1}^{M}$ is $\mathcal{L}$, with these classes interpreted over $\mathfrak{C}_{0}(M)$. Of course, most of the time, for type 3 premice $M$, we deal with $\mathfrak{C}_{0}(M)$, but in special circumstances we need to deal directly with $M$ instead, interpreting $\mathcal{L}$ over $M$ as above (in these circumstances we use simple embeddings and ultrapowers, as discussed below).

We also define the natural language $\mathcal{L}_{\text {nat }}^{M}$ of $M$ : if $M$ is passive, $\mathcal{L}_{\text {nat }}^{M}=$ $\mathcal{L} \backslash\left\{\dot{\widetilde{F}}, \dot{F}_{\downarrow}\right\}$; if $M$ is type $1 / 2, \mathcal{L}_{\text {nat }}=\mathcal{L}$, and if $M$ is type $3, \mathcal{L}_{\text {nat }}^{M}=\mathcal{L} \backslash\left\{\dot{F}_{\downarrow}\right\}$.

For the basic fine structural notions (soundness, solidity, $\mathrm{r} \Sigma_{n+1}$, etc), we follow Mitchell-Steel, as modified in $[13, \S 5]$. This modification involves three things. The first (and main one) is that we drop the parameter $u_{n}$ of $[3, \S 2]$, defining $p_{n+1}$ without reference to $u_{n}$. (Recall $u_{0}^{M}=\emptyset$ an if $n>0$ then $u_{n}^{M}=$ $\left(p_{n}^{M}, w_{n}^{M}, \widetilde{\rho}_{n-1}^{M}, u_{n-1}^{M}\right)$ where $w_{n}$ is the set of $n$-solidity witnesses (in the sense of [3]), $\widetilde{\rho}_{n-1}^{M}=\rho_{n-1}^{M}$ if $\rho_{n-1}^{M}<\rho_{0}^{M}$, and $\widetilde{\rho}_{n-1}^{M}=0$ otherwise.) The reader who prefers the original fine structure simply need put $u_{n}$ into all $\mathrm{r} \Sigma_{n+1}$ hulls and $\mathrm{r} \Sigma_{n+1}$ theories. By [13], this change does not have any significant impact; it just simplifies notation. The second is that, in the terminology of [3], we use only pure theories, not generalized theories. Thus (comparing with $[3$, Definition 2.3.6]), if $M$ is $n$-sound and $\omega<\rho_{n}^{M}$, we define the predicate $T_{n}^{M}$, where $n \geq 1$, as the set of tuples $(\alpha, q, t) \in \mathfrak{C}_{0}(M)$ with $\alpha<\rho_{n}^{M}$ and $q \in \mathfrak{C}_{0}(M)$ and $t=$ $\mathrm{Th}_{\mathrm{r} \Sigma_{n}}^{M}(\alpha \cup\{q\})$, where this denotes the pure $\mathrm{r} \Sigma_{n}$ theory; see below. This also has no significant impact, as explained in [3, Lemma 2.10]. The third is just terminological: for the definition of $(n+1)$-solidity for a structure $N$, we follow [18], not [3]; this is discussed below.

As described in $\S 2$, we also use $n$-lifting embeddings where weak $n$-embeddings are used classically (but this does not impact any basic definitions).

So, from now on we use the fine structural notions defined as in $[13, \S 5]$. Let $n<\omega$ and let $M$ be an $n$-sound premouse. For $i \leq n+1$ we write $\vec{p}_{i}^{M}=$

indexing, which is at the superstrong level. However, in this paper we do not need to consider Dodd-solidity. 
$\left(p_{1}^{M}, \ldots, p_{i}^{M}\right)$. Now suppose $\omega<\rho_{n}^{M}$, and let $X \subseteq \mathfrak{C}_{0}(P)$. Almost as in [12], Hull $_{n+1}^{M}(X)$ denotes the restriction of (the predicates of ) $\mathfrak{C}_{0}(M)$ to the points $y \in \mathfrak{C}_{0}(M)$ such that for some $\mathrm{r} \Sigma_{n+1}$ formula $\varphi$ and $\vec{x} \in X^{<\omega}, y$ is the unique $z \in$ $\mathfrak{C}_{0}(M)$ such that $\mathfrak{C}_{0}(M) \models \varphi(\vec{x}, z)$. (This is not exactly as in [12], because we do not automatically put $u_{n}^{M}$ into the hull.) And $\mathrm{cHull}_{n+1}^{M}(X)$ is the transitive collapse of this structure. Also, $\operatorname{Th}_{\mathrm{r} \Sigma_{n+1}}^{M}(X)$ denotes the $\mathrm{r} \Sigma_{n+1}$ theory of $\mathfrak{C}_{0}(M)$ in parameters in $X$ (that is, all pairs $(\varphi, \vec{x})$ such that $\varphi$ is an $\mathrm{r} \Sigma_{n+1}$ formula and $\vec{x} \in X^{<\omega}$ and $\mathfrak{C}_{0}(M) \models \varphi(\vec{x})$ ). This notation differs from [12], in that it denotes a pure theory, not a generalized theory, in the terminology of [3]; we have no need for generalized theories. If $\omega<\rho_{n+1}^{M}$, we then define $T_{n+1}^{M}$ as stated above, and define $\mathrm{r} \Sigma_{n+2}$ from this as in [3].

Given $q \in\left[\rho_{0}^{M}\right]^{<\omega}$ and $\alpha \in q$, the $(n+1)$-solidity witness for $(M, q, \alpha)$ (or just for $(q, \alpha))$ is $w_{q, \alpha}^{M}=\operatorname{cHull}_{n+1}^{M}\left(\alpha \cup\left\{q^{\prime}, \vec{p}_{n}^{M}\right\}\right.$ ) where $q^{\prime}=q \backslash(\alpha+1)$. A generalized $(n+1)$-solidity witness for $(M, q, \alpha)$ is the obvious adapatation from $[18, \S 1.12]$. A (generalized) $(n+1)$-solidity witness for $(M, q)$ is one for $(M, q, \alpha)$, for some $\alpha \in q$; a witness for $M$ is one for $\left(M, p_{n+1}^{M}\right)$. We say that $(M, q)$ is $(n+1)$-solid iff $w_{q, \alpha}^{M} \in M$ for each $\alpha \in q$. We say that $M$ is $(n+1)$-solid iff $\left(M, p_{n+1}^{M}\right)$ is $(n+1)$-solid. ${ }^{10}$ Given $\rho \in\left[\rho_{n+1}^{M}, \rho_{n}^{M}\right]$, we say that $M$ is $\rho$-solid iff $\left(M, p_{n+1}^{M} \backslash \rho\right)$ is $(n+1)$-solid, and that $M$ is $\rho$-sound iff $M$ is $\rho$-solid and $M=\operatorname{Hull}_{n+1}^{M}\left(\rho \cup p_{n+1}^{M}\right)$. We say $M$ is $(n+1)$-sound iff $M$ is $\rho_{n+1}^{M}$-sound. ${ }^{11}$ For $\delta \in\left[\rho_{n+1}^{M}, \rho_{0}^{M}\right]$, the $\delta$-core of $M$ is $\operatorname{cHull}_{n+1}^{M}\left(\delta \cup\left\{\vec{p}_{n+1}^{M}\right\}\right)$.

\subsubsection{Extenders and ultrapowers}

Our use of the term extender is standard for inner model theory. Extenders need not be total over $V$, and need not yield wellfounded ultrapowers. We use the term basically as in [12] (see its introduction), except that in $\S 6$ we must also consider extenders over coarse structures.

Given an extender $E$ over $M, U=\operatorname{Ult}(M, E)$ denotes the ultrapower of $M$ by $E$, computed using functions in $M$; this is formed directly, without any squashing, whatever $M$ is, and if $M$ is an amenable structure, its predicates are shifted piece-wise as usual. Such an ultrapower is called simple. We write $i_{E}^{M}$ for the ultrapower embedding $i_{E}^{M}: M \rightarrow U$. We may abbreviate $i_{E}^{M}$ by $i_{E}$. We write $\operatorname{ms}(E)$ for the measure space of $E$; that is, the supremum of all $\kappa+1$ such that $\kappa \in \operatorname{dom}\left(i_{E}\right)$ and $\left(\sup i_{E} " \kappa\right)<\operatorname{lh}(E)$ ( $E$ might be long). If $E$ is short and $\kappa=\operatorname{cr}(E)$, we say that $E$ is weakly amenable (to $M)$ iff $\mathcal{P}(\kappa) \cap M=\mathcal{P}(\kappa) \cap U$. Note that if $M$ is an iterable premouse, then by condensation, this is equivalent to saying that $M \| \kappa^{+M}=U \mid \kappa^{+U}$. Now suppose $M$ is an $n$-sound premouse, $E$

\footnotetext{
${ }^{10}$ Regarding the $(n+1)$-solidity of $M$, this follows the (analogous) terminology of Zeman [18, p.44, Definition prior to Lemma 1.12.5], but not that of Mitchell-Steel [3, Definition 2.8.2] and [16, Definition 2.15]; Mitchell-Steel demands universality as one of the conditions for solidity, whereas Zeman does not (and neither do we). In [18], see also its Lemma 5.1.7(c), the paragraph following Corollary 5.1.8, Theorem 5.2.1, Lemma 9.2.14, and Theorem 9.3.1, which treat the $(n+1)$-solidity of a structure $M$ and $(n+1)$-universality for $\left(M, p_{n+1}^{M}\right)$ separately.

11 The terminology $(n+1)$-sound follows Mitchell-Steel, not Zeman, as Zeman does not incorporate $(n+1)$-solidity into $(n+1)$-soundness.
} 
is short and $\operatorname{cr}(E)<\rho_{n}^{M}$. Then $\operatorname{Ult}_{n}(M, E)$ denotes the degree $n$ fine structural ultrapower of $M$ by $E$, and $i_{E}^{M, n}$ the ultrapower map. We may abbreviate $i_{E}^{M, n}$ by $i_{E}^{M}$ or $i_{E}$. Given $a \in[\operatorname{lh}(E)]^{<\omega}$ and an $\mathrm{r} \sum_{n}^{M}$ function $f,[a, f]_{E}^{M, n}$ denotes the object represented by the pair $(a, f)$ in $\operatorname{Ult}_{n}(M, E)$. Recall that if $M$ is type 3 , then $\operatorname{Ult}_{n}(M, E)=\operatorname{Ult}_{n}\left(M^{\text {sq }}, E\right)^{\text {unsq }}$. If $M$ is type 3 then we let $\mathfrak{C}_{-1}(M)=$ $\mathfrak{C}_{0}(M)$, and for an extender $E$ over $\mathfrak{C}_{0}(M)$, let $\operatorname{Ult}_{-1}(M, E)=\operatorname{Ult}_{0}(M, E)$.

\subsubsection{Embeddings}

Given structures $X, Y$, if context determines an obvious natural embedding $i: X \rightarrow Y$, we sometimes write $i_{X Y}$ for $i$.

Let $M, N$ be segmented-premice. A simple embedding $\pi: M \rightarrow N$ is a function $\pi$ with $\operatorname{dom}(\pi)=\lfloor M\rfloor$ and $\operatorname{cod}(\pi)=\lfloor N\rfloor$, such that $\pi$ is $\Sigma_{0}$-elementary with respect to $\mathcal{L}$. (Note that if $M$ is active then $\pi(\operatorname{lgcd}(M))=\operatorname{lgcd}(N)$, because the amenable predicates for $F^{M}$ and $F^{N}$ determine the largest cardinal.) If $M, N$ are type 3 premice, a squashed embedding $\pi: M \rightarrow N$ is, literally, an $\mathrm{r} \Sigma_{0}$-elementary function $\pi: \mathfrak{C}_{0}(M) \rightarrow \mathfrak{C}_{0}(N)$.

Let $\pi: M \rightarrow N$ be simple. If $M$ is passive then then $\psi_{\pi}$ denotes $\pi$. If $M$ is active then $\psi_{\pi}: \operatorname{Ult}\left(M, F^{M}\right) \rightarrow \operatorname{Ult}\left(N, F^{N}\right)$ denotes the embedding induced by $\pi$ (via the Shift Lemma). Now let $\pi: M \rightarrow N$ be squashed (so $M, N$ are type $3)$. Then $\psi_{\pi}: \operatorname{Ult}_{0}\left(M, F^{M}\right) \rightarrow \operatorname{Ult}_{0}\left(N, F^{N}\right)$ denotes the embedding induced by $\pi$. So $\pi \subseteq \psi_{\pi}$ in both cases.

Let $\pi: M \rightarrow N$ be simple or squashed. We say $\pi$ is (i) $\nu$-preserving iff $\pi$ is simple or $\psi_{\pi}\left(\nu\left(F^{M}\right)\right)=\nu\left(F^{N}\right)$, (ii) $\nu$-high iff $\pi$ is squashed and $\psi_{\pi}\left(\nu\left(F^{M}\right)\right)>$ $\nu\left(F^{N}\right)$, (iii) $\nu$-low iff $\pi$ is squashed and $\psi_{\pi}\left(\nu\left(F^{M}\right)\right)<\nu\left(F^{N}\right)$, (iv) $\iota$-preserving iff either $M, N$ are passive or $\psi_{\pi}\left(\iota\left(F^{M}\right)\right)=\iota\left(F^{N}\right)$, (v) c-preserving iff for all $\alpha, \alpha$ is a cardinal of $M$ iff $\pi(\alpha)$ is a cardinal of $N$.

1.1 Remark. Let $\pi: M \rightarrow N$ be a squashed embedding (so $M, N$ are type 3). Easy elementarity considerations show that if $\pi$ is $\mathrm{r} \Sigma_{1}$-elementary then $\pi$ is non- $\nu$-low, and if $\mathrm{r} \Sigma_{2}$-elementary then $\nu$-preserving. Suppose $\pi$ is $\nu$-low and let $\nu^{\prime}=\psi_{\pi}\left(\nu\left(F^{M}\right)\right)$. Then $\nu^{\prime}<\nu\left(F^{N}\right)$ is an $N$-cardinal, so by ISC, there is $N^{\prime} \triangleleft N$ with $F^{N^{\prime}}\left\lceil\nu^{\prime}=F^{N} \uparrow \nu^{\prime}\right.$. Note that we get an $\mathrm{r} \Sigma_{0}$-elementary $\pi^{\prime}: \mathfrak{C}_{0}(M) \rightarrow \mathfrak{C}_{0}\left(N^{\prime}\right)$ with the same graph as $\pi$, and $\pi^{\prime}$ is $\nu$-preserving.

Let $\pi: \mathfrak{C}_{0}(M) \rightarrow \mathfrak{C}_{0}(N)$ be $\mathrm{r} \Sigma_{0}$-elementary where $M, N$ are $n$-sound. We say $\pi$ is (i) $p_{n+1}$-preserving iff $\pi\left(p_{n+1}^{M}\right)=p_{n+1}^{N}$, (ii) $\vec{p}_{n+1}$-preserving iff $\pi\left(\vec{p}_{n+1}^{M}\right)=$ $\vec{p}_{n+1}^{N}$, (iii) $\rho_{j}$-preserving iff either $\pi\left(\rho_{j}^{M}\right)=\rho_{j}^{N}$ or $\left[\rho_{j}^{M}=\rho_{0}^{M}\right.$ and $\left.\rho_{j}^{N}=\rho_{0}^{N}\right]$.

\subsubsection{Iteration trees and iterability}

Let $\mathcal{T}$ be an iteration tree of length $\operatorname{lh}(\mathcal{T})=\lambda$. The objects associated to $\mathcal{T}$ we write as: tree order $<^{\mathcal{T}}$, drop-set $\mathscr{D}^{\mathcal{T}}$ (the nodes where drops in model occur), models $M_{\alpha}^{\mathcal{T}}$, degrees $\operatorname{deg}_{\alpha}^{\mathcal{T}}$, embeddings $i_{\alpha \beta}^{\mathcal{T}}$ and $i_{\alpha \beta}^{* \mathcal{T}}$ (defined where appropriate), exit extenders $E_{\alpha}^{\mathcal{T}}$, exit models $\operatorname{ex}_{\alpha}^{\mathcal{T}}=M_{\alpha}^{\mathcal{T}} \mid \operatorname{lh}\left(E_{\alpha}^{\mathcal{T}}\right)$, ultrapower domains $M_{\alpha+1}^{* \mathcal{T}}$ (so if $\mathcal{T}$ is fine structural and $d=\operatorname{deg}_{\alpha+1}^{\mathcal{T}}$ then $M_{\alpha+1}^{\mathcal{T}}=\operatorname{Ult}_{d}\left(M_{\alpha+1}^{* \mathcal{T}}, E_{\alpha}^{\mathcal{T}}\right)$ ), and associated ultrapower maps $i_{\alpha+1}^{* \mathcal{T}}: M_{\alpha+1}^{* \mathcal{T}} \rightarrow M_{\alpha+1}^{\mathcal{T}}\left(\right.$ so $\left.i_{\alpha+1, \beta}^{* \mathcal{T}}=i_{\alpha+1, \beta}^{\mathcal{T}} \circ i_{\alpha+1}^{* \mathcal{T}}\right)$, $\kappa_{\alpha}^{\mathcal{T}}=\operatorname{cr}\left(E_{\alpha}^{\mathcal{T}}\right)$ and $\nu_{\alpha}^{\mathcal{T}}=\nu\left(E_{\alpha}^{\mathcal{T}}\right)$. We write $\operatorname{pred}^{\mathcal{T}}(\alpha+1)$ for the $<^{\mathcal{T}}$-predecessor 
of $\alpha+1$, and given $\alpha<^{\mathcal{T}} \beta$, $\operatorname{succ}^{\mathcal{T}}(\alpha, \beta)$ denotes the least $\gamma \in(\alpha, \beta]_{\mathcal{T}}$. If $\operatorname{lh}(\mathcal{T})=\theta+1$, then $M_{\infty}^{\mathcal{T}}=M_{\theta}^{\mathcal{T}}, b^{\mathcal{T}}=[0, \infty]_{\mathcal{T}}=[0, \theta]_{\mathcal{T}}$, and if there is no drop along $b^{\mathcal{T}}$ then $i^{\mathcal{T}}=i_{0 \infty}^{\mathcal{T}}=i_{0 \theta}^{\mathcal{T}}$, etc.

Let $n \leq \omega$ and $M$ be an $n$-sound premouse. The notion $n$-maximal iteration tree $\mathcal{T}$ on $M$ is defined basically as in [16, Definition 3.4], or [3, Definition 6.1.2], but we must adapt these definitions to superstrongs. That is, $\operatorname{deg}_{0}^{\mathcal{T}}=n$ and for $\alpha+1<\operatorname{lh}(\mathcal{T})$, letting $\kappa=\kappa_{\alpha}^{\mathcal{T}}$, we have: (i) $\operatorname{lh}\left(E_{\beta}^{\mathcal{T}}\right) \leq \operatorname{lh}\left(E_{\alpha}^{\mathcal{T}}\right)$ for all $\beta<\alpha$; (ii) $\operatorname{pred}^{\mathcal{T}}(\alpha+1)$ is the least $\beta$ such that $\kappa<\nu_{\beta}^{\mathcal{T}}$; (iii) $M_{\alpha+1}^{* \mathcal{T}}$ is the largest $N \unlhd M_{\beta}^{\mathcal{T}}$ such that $E_{\alpha}^{\mathcal{T}}$ measures $\mathcal{P}(\kappa) \cap N$; and (iv) $\operatorname{deg}^{\mathcal{T}}(\alpha+1)$ is the largest $d \leq \omega$ such that $\kappa<\rho_{d}\left(M_{\alpha+1}^{* \mathcal{T}}\right)$ and either $[0, \alpha+1]_{\mathcal{T}}$ drops or $d \leq n$. We will also extend the notion $n$-maximal iteration tree to trees on premouse-related structures. An iteration tree is degree-maximal if $n$-maximal for some $n \leq \omega$.

For $\theta \leq \mathrm{OR}$, the notions $(n, \theta)$-iteration strategy for and $(n, \theta)$-iterability of $M$ are as in [16, Definition 3.9] (but using $n$-maximality defined as above). For $(n, \alpha, \theta)^{*}$-iteration strategy and $(n, \alpha, \theta)^{*}$-iterable see [14, p. 1202]. ${ }^{12}$

If $\mathcal{T}$ is padded, unless otherwise specified, if $\beta=\operatorname{pred}^{\mathcal{T}}(\alpha+1)$ then $E_{\beta}^{\mathcal{T}} \neq \emptyset$.

\section{Fine structural preliminaries}

2.1 Definition. Let $H, M$ be $k$-sound premice with $\rho_{k}^{H}, \rho_{k}^{M}>\omega$. We say an embedding $\pi: \mathfrak{C}_{0}(H) \rightarrow \mathfrak{C}_{0}(M)$ is $k$-lifting iff $\pi$ is $\mathrm{r} \Sigma_{0}$-elementary with respect to the natural language $\mathcal{L}_{\text {nat }}^{H}$ of $H$ (see $\S 1$ ) and if $k>0$ then $\pi$ " $T_{k}^{H} \subseteq T_{k}^{M}$.

A $k$-lifting embedding is similar to a $\Sigma_{0}^{(k)}$-preserving embedding of [18]. Note that $H, M$ may have different natural languages; maybe $\mathcal{L}_{\text {nat }}^{H} \subsetneq \mathcal{L}_{\text {nat }}^{M}$.

2.2 Lemma. Let $H, M, k$ be as in 2.1 and let $\pi: \mathfrak{C}_{0}(H) \rightarrow \mathfrak{C}_{0}(M)$. Then:

1. $\pi$ is k-lifting iff for every $\mathrm{r} \Sigma_{k+1}$ formula $\varphi \in \mathcal{L}_{\text {nat }}^{H}$ and $x \in \mathfrak{C}_{0}(H)$, if $\mathfrak{C}_{0}(H) \models \varphi(x)$ then $\mathfrak{C}_{0}(M) \models \varphi(\pi(x))$.

2. If $\pi$ is $k$-lifting and $H, M$ have different types then $k=0, H$ is passive and $M$ is active.

3. If $k>0$ and $\pi$ is $k$-lifting then $\pi$ is $\mathrm{r} \Sigma_{k}$-elementary, $(k-1)$-lifting, $c$ preserving.

4. Suppose $k>1$ and $\pi$ is $\mathrm{r} \Sigma_{k}$-elementary. Then $\pi$ is $p_{k-2}$-preserving and $\rho_{k-2}$-preserving, and if $\rho_{k-1}^{H}<\rho_{0}^{H}$ then $\pi\left(p_{k-1}^{H}\right)=p_{k-1}^{M} \backslash \pi\left(\rho_{k-1}^{H}\right)$ and $\left(\sup \pi " \rho_{k-1}^{H}\right) \leq \rho_{k-1}^{M} \leq \pi\left(\rho_{k-1}^{H}\right)$.

5. If $k>0$ and $\pi$ is $\mathrm{r} \Sigma_{k}$ elementary and $p_{k-1}$-preserving then $\pi\left(p_{k}^{H}\right) \leq p_{k}^{M}$.

\footnotetext{
${ }^{12}$ The relevant trees are stacks of degree-maximal trees, each of length $\leq \theta$, starting with $n$-maximal. The superscript- $*$ has the effect that if in some round $<\alpha$, a degree-maximal tree is produced which has length $\theta$, then the game stops there immediately, and (if it has wellfounded models) player II has won.
} 
6. The Shift Lemma holds with weak $k$ - replaced by $k$-lifting, or by $k$-lifting c-preserving.

Proof. Parts 1-3 are straightforward. For part 4 , use $(k-1)$-solidity witnesses for $p_{k-1}$. For part 5 use the fact that if $t$ is a $k$-solidity witness for $\left(H, p_{k}^{H}\right)$, then $\pi(t)$ is a generalized $k$-solidity witness for $\left(M, \pi\left(p_{k}^{H}\right)\right)$.

Part 6: We adopt the notation of [3, Lemma 5.2] (with $n=k$ ). Let $\bar{F}=F^{\bar{N}}$ and $\bar{U}=\operatorname{Ult}_{k}(\bar{M}, \bar{F})$ and $U=\operatorname{Ult}_{k}\left(M, F^{N}\right)$. Define the map $\sigma: \mathfrak{C}_{0}(\bar{U}) \rightarrow \mathfrak{C}_{0}(U)$ as there. It is straightforward to see that $\sigma$ is $\mathrm{r} \Sigma_{k}$-elementary. Suppose $k>0$. Let us observe that $\sigma^{\prime \prime} T_{k}^{\bar{U}} \subseteq T_{k}^{U}$. Let $t \in T_{k}^{\bar{U}}, x \in \bar{U}$ and $\alpha<\rho_{k}^{\bar{U}}$ with $t=\operatorname{Th}_{\mathrm{r} \Sigma_{k}}^{\bar{U}}(\alpha \cup\{x\})$. Let $y \in \bar{M}$ and $a \in \nu(\bar{F})^{<\omega}$ with $x \in \operatorname{Hull}_{k}^{\bar{U}}(i \bar{M}(y) \cup a)$. Let $\beta<\rho_{k}^{\bar{M}}$ be such that $\beta \geq \operatorname{cr}(\bar{F})$ and $i_{\bar{F}}^{\bar{M}}(\beta) \geq \alpha$. Let $u=\operatorname{Th}_{\mathrm{r} \Sigma_{k}}^{\bar{M}}(\beta \cup\{y\})$. Then $t$ is easily computed from $u^{\prime}=i_{\bar{F}}^{\bar{M}}(u)$, and by commutativity, $\sigma\left(u^{\prime}\right) \in T_{k}^{U}$. It follows that $\sigma(t) \in T_{k}^{U}$, as required.

2.3 Remark. Clearly for $k<\omega$, any $\mathrm{r} \Sigma_{k+1}$-elementary embedding is $k$-lifting. The author does not know whether "weak $k$-" implies " $k$-lifting", or vice versa. We will not deal with weak $k$-embeddings in this paper.

Standard arguments show that the copying construction propagates $k$-lifting c-preserving embeddings. (But this may be false for weak $k$-embeddings; see [10].) Almost standard arguments show that $k$-lifting embeddings are propagated. That is, suppose $\pi: H \rightarrow M$ is $k$-lifting, and let $\mathcal{T}$ be a $k$-maximal iteration tree on $H$. We can define $\mathcal{U}=\pi \mathcal{T}$ as usual, assuming it has wellfounded models. Let $H_{\alpha}=M_{\alpha}^{\mathcal{T}}$ and $M_{\alpha}=M_{\alpha}^{\mathcal{U}}$. Using the Shift Lemma as usual, we get $\pi_{\alpha}: H_{\alpha} \rightarrow M_{\alpha}$ for each $\alpha<\operatorname{lh}(\mathcal{T})$, and $\pi_{\alpha}$ is $\operatorname{deg}^{\mathcal{T}}(\alpha)$-lifting, and if $\pi$ is c-preserving, then so is $\pi_{\alpha}$. Let us just mention the extra details when $\pi$ fails to be c-preserving. In this case, $k=0$ and $H$ is passive. Suppose that $E_{0}^{\mathcal{T}}$ is total over $H$, and let $\kappa=\operatorname{cr}\left(E_{0}^{\mathcal{T}}\right)$. Suppose that $\left(\kappa^{+}\right)^{H}<\mathrm{OR}^{H}$ but $\pi\left(\left(\kappa^{+}\right)^{H}\right)$ is not a cardinal of $M$. Then $\mathcal{U}$ drops in model at 1 , but $\mathcal{T}$ does not. Note though that $\operatorname{rg}(\pi) \subseteq M_{1}^{* \mathcal{U}}$ and $\pi: H \rightarrow M_{1}^{* \mathcal{U}}$ is 0-lifting (even if $M_{1}^{* \mathcal{U}}$ is active). So we can still produce $\pi_{1}: H_{1} \rightarrow M_{1}$ via the Shift Lemma. This situation generalizes to an arbitrary $\alpha$ in place of 0 , when $\mathcal{T}$ does not drop in model along $[0, \alpha+1]_{\mathcal{T}}$. The other details are as usual. Moreover, if (i) $[0, \alpha]_{\mathcal{T}}$ drops in model or (ii) $\operatorname{deg}^{\mathcal{T}}(\alpha) \leq k-2$ or (iii) $\operatorname{deg}^{\mathcal{T}}(\alpha)=k-1$ and $\pi$ is $p_{k-1}$-preserving, then $\pi_{\alpha}$ is a near $\operatorname{deg}^{\mathcal{T}}(\alpha)$-embedding; this uses the argument in [4].

2.4 Lemma. Let $k \geq 0$, let $\pi: H \rightarrow M$ be $k$-lifting where $H, M$ have the same type, and let $\rho_{k+1}^{H} \leq \rho \leq \rho_{k}^{H}$. Then:

1. If $p_{k-1}^{M}, p_{k}^{M} \in \operatorname{rg}(\pi)$ and $\rho_{k}^{M}=\sup \pi^{\prime} \rho_{k}^{H}$ then $\pi$ is a $k$-embedding.

2. If $H$ is $\rho$-sound, $\pi \uparrow \rho \in M$ and $\pi$ is not a k-embedding then $H, \pi \uparrow \rho_{k}^{H} \in M$.

Proof. Part 1: This is fairly routine. By 2.2, we have $\pi\left(p_{k-1}^{H}\right)=p_{k-1}^{M}$. The $\mathrm{r} \Sigma_{k+1}$-elementarity of $\pi$ follows from this, together with the facts that $\pi$ is $k$ lifting, $p_{k}^{M} \in \operatorname{rg}(\pi)$ and $\pi " \rho_{k}^{H}$ is unbounded in $\rho_{k}^{M}$. Now let $\pi(q)=p_{k}^{M}$. Then 
$p_{k}^{H} \leq q$ by 2.2 , and $q \leq p_{k}^{H}$ by $\mathrm{r} \Sigma_{k}$ elementarity. And $\pi$ is $\rho_{k-1}$-preserving by 2.2 and $\mathrm{r} \Sigma_{k+1}$-elementarity. So $\pi$ is a $k$-embedding.

Part 2: Suppose $\sup \left(\pi^{\prime \prime} \rho_{k}^{H}\right)<\rho_{k}^{M}$. We use a stratifaction of $\mathrm{r} \Sigma_{k+1}$ truth like in [3, §2]. Assuming familiarity with this, here is a sketch. Let $\alpha=\sup \pi^{\prime \prime} \rho_{k}^{H}$. Then the theory $t=\operatorname{Th}_{\mathrm{r} \Sigma_{k}}^{M}\left(\alpha \cup \pi\left(\vec{p}_{k}^{H}\right)\right)$ is in $M$. Moreover, for any $\mathrm{r} \Sigma_{k+1}$ formula $\varphi$ and $\vec{\gamma} \in \rho^{<\omega}$, we have $H \models \varphi\left(\vec{\gamma}, \vec{p}_{k+1}^{H}\right)$ iff there is $\beta<\alpha$ such that $t \uparrow\left(\beta \cup \pi\left(\vec{p}_{k}^{H}\right)\right)$ is "above" a witness to $\varphi\left(\pi(\vec{\gamma}), \pi\left(\vec{p}_{k+1}^{H}\right)\right)$ (see [3, §2]). So the theory $\operatorname{Th}_{\mathrm{r} \Sigma_{k+1}}^{H}\left(\rho \cup \vec{p}_{k+1}^{H}\right)$ is computable from $t$ and $\pi \uparrow \rho$, so $H \in M$. A little more work gives $\pi\left\lceil\rho_{k}^{H} \in M\right.$, as desired.

Suppose now $\pi\left(p_{k-1}^{H}\right)=p_{k-1}^{M}$ but $\pi\left(p_{k}^{H}\right) \neq p_{k}^{M}$. Then $\pi\left(p_{k}^{H}\right)<p_{k}^{M}$, by 2.2 . Again $t \in M$ ( $t$ as above), as $t$ is computed from a $k$-solidity witness. The rest is the same.

Now suppose that $k>1$ and $\pi\left(p_{k-1}^{H}\right) \neq p_{k-1}^{M}$. By 2.2, then $\pi\left(p_{k-1}^{H}\right)<p_{k-1}^{M}$. We may assume $\alpha=\rho_{k}^{M}=\sup \pi " \rho_{k}^{H}$.

Claim. Let $\varphi$ be an $\mathrm{r} \Sigma_{k}$ formula, let $x \in H$ and $\vec{\gamma} \in \alpha^{<\omega}$. If $M \models \varphi(\pi(x), \vec{\gamma})$ then there is $\varepsilon<\rho_{k-1}^{M}$, with $\max (\vec{\gamma})<\varepsilon$, such that the theory

$$
\operatorname{Th}_{\mathrm{r} \Sigma_{k-1}}^{M}\left(\varepsilon \cup\left\{\pi\left(x, \vec{p}_{k-1}^{H}\right)\right\}\right)
$$

is "above" a witness to $\varphi(\pi(x), \vec{\gamma})$.

Proof. Let $\delta<\rho_{k}^{H}$ be such that $\pi(\delta)>\max (\vec{\gamma})$. Let $v=\operatorname{Th}_{\mathrm{r} \Sigma_{k}}^{H}\left(\delta \cup\{x\} \cup \vec{p}_{k-1}^{H}\right)$. Note then that for all $\vec{\xi} \in \delta^{<\omega}$,

$$
\left(\varphi,\left(\vec{\xi}, x, \vec{p}_{k-1}^{H}\right)\right) \in v \quad \Longrightarrow \quad\left(\psi_{\varphi},\left(\vec{\xi}, x, \vec{p}_{k-1}^{H}\right)\right) \in v
$$

where $\psi_{\varphi}\left(\vec{\xi}, x, \vec{p}_{k-1}^{H}\right)$ asserts 'There is $\varepsilon<\rho_{k-1}$, with $\max (\vec{\xi})<\varepsilon$, such that the $\mathrm{r} \Sigma_{k-1}$ theory in parameters $\varepsilon \cup\{x\} \cup \vec{p}_{k-1}^{H}$ is "above" a witness to $\varphi\left(\vec{\xi}, x, \vec{p}_{k-1}^{H}\right)$ '. (Here the assertion that $\varepsilon<\rho_{k-1}$ does not require the parameter $\rho_{k-1}^{H}$. For note that the assertion " $\dot{u}<\rho_{k-1}$ ", in the free variable $\dot{u}$, is $\mathrm{r} \Sigma_{k}$ without parameters, because it is just the assertion " $\dot{u} \in \mathrm{OR}$ and there is $t \in T_{k-1}$ such that $t$ is a theory in parameters from $\dot{u}$ ".) But then the same fact holds regarding $\pi(v)$, and since $\pi$ is $k$-liftng, this proves the claim.

By $(k-1)$-solidity, $u=\operatorname{Th}_{\mathrm{r} \Sigma_{k-1}}^{M}\left(\rho_{k-1}^{M} \cup \pi\left(\vec{p}_{k-1}^{H}\right)\right)$ is in $M$. Define $t$ as before. By the claim, from $u$ we compute $t$, so $t \in M$. The rest is now as before.

2.5 Definition. Let $Q$ be a $k$-sound premouse. Let $\widetilde{\mathfrak{C}}_{0}(Q)=\mathfrak{C}_{0}(Q)$, and for $k>0$, let $\widetilde{\mathfrak{C}}_{k}(Q)=\left(Q \| \rho_{k}(Q), T^{\prime}\right)$, where $T=\operatorname{Th}_{\mathrm{r} \Sigma_{k}}^{Q}\left(\rho_{k} \cup \vec{p}_{k}^{Q}\right)$, and $T^{\prime}$ is given from $T$ by substituting $\vec{p}_{k}^{Q}$ for a constant symbol $c$.

2.6 Definition. Let $k \geq 0$. Let $Q$ be a $k$-sound premouse with $\rho_{k}^{Q}>\omega$. We say that $\left(U, \sigma^{*}\right)$ is $k$-suitable for $Q$ iff (i) $U, \sigma^{*} \in Q \| \rho_{k}^{Q}$, (ii) $U$ is a $k$-sound premouse with $\rho_{k}^{U}>\omega$ and (iii) $\sigma^{*}: \widetilde{\mathfrak{C}}_{k}(U) \rightarrow \widetilde{\mathfrak{C}}_{k}(Q)$ is $\Sigma_{0}$-elementary. 
2.7 Remark. Clearly, if $\left(U, \sigma^{*}\right)$ is $k$-suitable for $Q$ then $\sigma^{*}$ extends uniquely to a $\vec{p}_{k}$-preserving $k$-lifting $\sigma: U \rightarrow Q$, and moreover, $\sup \sigma " \rho_{k}^{U}<\rho_{k}^{Q}$. Conversely, if $\sigma: U \rightarrow Q$ is $\vec{p}_{k}$-preserving $k$-lifting and $\sup \sigma^{\prime \prime} \rho_{k}^{U}<\rho_{k}^{Q}$ and $\sigma^{*}=\sigma \uparrow\left(U \| \rho_{k}^{U}\right)$ with $\sigma^{*} \in Q$, then $\left(U, \sigma^{*}\right)$ is $k$-suitable for $Q$.

2.8 Lemma. Let $k \geq 0$. Then there is an $\mathrm{r} \Sigma_{k+1}$ formula $\varphi_{k}$ such that for all $k$-sound premice $Q$ with $\omega<\rho_{k}^{Q}$, and all $U, \sigma^{*} \in Q$, we have

$$
Q \models \varphi_{k}\left(U, \sigma^{*}, \vec{p}_{k}^{Q}\right) \Longleftrightarrow\left(U, \sigma^{*}\right) \text { is k-suitable for } Q .
$$

Proof. We assume $k>0$ and leave the other case to the reader. The most complex clause of $\varphi_{k}$ says "There is $\alpha<\rho_{k}^{Q}$ such that letting $t=\operatorname{Th}_{\mathrm{r} \Sigma_{k}}^{Q}\left(\alpha \cup \vec{p}_{k}^{Q}\right)$, then for each $\beta<\rho_{k}^{U}$, letting $u=\operatorname{Th}_{\mathrm{r} \Sigma_{k}}^{U}\left(\beta \cup \vec{p}_{k}^{U}\right)$, and letting $t^{\prime}, u^{\prime}$ be given from $t, u$ by substituting $\vec{p}_{k}^{Q}, \vec{p}_{k}^{U}$ for the constant $c$, we have $\sigma^{*}\left(u^{\prime}\right) \subseteq t^{\prime \prime}$. This statement is $\mathrm{r} \Sigma_{k+1}$. The rest is clear.

2.9 Definition. Let $m \geq 0$ and let $M$ be a segmented-premouse. Then $M$ is $m$-sound iff either $m=0$ or $M$ is an $m$-sound premouse.

2.10 Definition. Let $r \geq 0$ and let $R$ be an $r$-sound premouse. Then we say that suitable condensation holds at $(R, r)$ iff for every $\left(H, \pi^{*}\right)$, if $\left(H, \pi^{*}\right)$ is $r$-suitable for $R, H$ is $(r+1)$-sound and $\operatorname{cr}(\pi) \geq \rho=\rho_{r+1}^{H}$, then either $H \triangleleft R$, or $R \mid \rho$ is active with extender $F$ and $H \triangleleft \operatorname{Ult}(R \mid \rho, F)$.

Let $m \geq 0$ and let $M$ be an $m$-sound segmented-premouse. We say that suitable condensation holds below $(M, m)$ iff for every $R \unlhd M$ and $r<\omega$ such that either $R \triangleleft M$ or $r<m$, suitable condensation holds at $(R, r)$. We say that suitable condensation holds through $(M, m)$ iff $M$ is a premouse ${ }^{13}$ and suitable condensation holds below and at $(M, m)$.

The following lemma follows easily from 2.8 :

2.11 Lemma. Let $m \geq 0$. Then there is an $\mathrm{r} \Pi_{\max (m, 1)}$ formula $\Psi_{m}$ such that for all $m$-sound segmented-premice $M$, suitable condensation holds below $(M, m)$ iff $M \models \Psi_{m}\left(\vec{p}_{m-1}^{M}\right)$, where $\vec{p}_{-1}^{M}=\emptyset$. Moreover, if $M$ is a premouse, then suitable condensation holds through $(M, m)$ iff $M \models \Psi_{m+1}\left(\vec{p}_{m}^{M}\right){ }^{14}$

2.12 Remark. Our proof of condensation from normal iterability (5.2) will use our analysis of bicephali and cephalanxes (§3). This analysis will depend on the premice involved satisfying enough condensation, at levels lower in model or degree. As we will only have normal iterability, we can't appeal to the standard condensation theorem for this. One could get arrange everything inductively, proving condensation and analysing bicephali and cephalanxes simultaneously. But it is simpler to use the following lemmas, which will be generalized by 5.2 .

\footnotetext{
${ }^{13}$ We could have formulated this more generally for segmented-premice, but doing so would have increased notational load, and we do not need such a generalization.

${ }^{14}$ This clause only adds something because we do not assume that $M$ is $(m+1)$-sound.
} 
2.13 Lemma (Condensation for $\omega$-sound mice). Let $h \leq m<\omega$ and $H, M$ be premice. Suppose $H$ is $(h+1)$-sound, $M$ is $(m+1)$-sound, $M$ is $\left(m, \omega_{1}+1\right)$ iterable, and either $\rho_{m+1}^{M}=\omega$ or $m \geq h+5$. Suppose $\pi: H \rightarrow M$ is h-lifting $\vec{p}_{h}$-preserving and $\operatorname{cr}(\pi) \geq \rho=\rho_{h+1}^{H}$. Then either $H \unlhd M$ or $M \mid \rho$ is active with $H \triangleleft \operatorname{Ult}(M \mid \rho, G)$.

Proof. Let $\pi$, etc, be a counterexample. Let $\pi^{*}=\pi\left\lceil\left(H \| \rho_{h}^{H}\right)\right.$.

We claim $H \in M$. Suppose not. By 2.4, $\pi$ is an $h$-embedding, and $\rho \geq$ $\rho_{h+1}^{M}$. Note $\pi\left(p_{h+1}^{H}\right) \leq p_{h+1}^{M} \backslash \rho$ (use generalized solidity witnesses). If $\pi\left(p_{h+1}^{H}\right)<$ $p_{h+1}^{M} \backslash \rho$ we are done. Otherwise $\rho_{h+1}^{M} \cup p_{h+1}^{M} \subseteq \operatorname{rg}(\pi)$, so $H=M$, contradiction.

We may assume $\rho_{m+1}^{M}=\omega$, by replacing $M$ with $\bar{M}=\mathrm{cHull}_{m+1}^{M}\left(\vec{p}_{m}\right)$ : all relevant facts pass to $\bar{M}$ as $H \in M, \operatorname{cr}(\pi) \geq \rho$ and by 2.2(1). Now use almost the usual condensation proof, but when comparing the phalanx $(M, H, \rho)$ with $M$, use an $(m, h)$-maximal tree on $(M, H, \rho), m$-maximal on $M$. As $H \in M$, and using fine structure in place of weak Dodd-Jensen, this gives contradiction.

2.14 Lemma (Suitable condensation). Let $M$ be an $m$-sound, $\left(m, \omega_{1}+1\right)$ iterable segmented-premouse. Then suitable condensation holds below $(M, m)$, and if $M$ is a premouse, through $(M, m)$.

Proof. If $M$ is not a pm, use 2.13. Suppose $M$ is a pm. By 2.11, we may assume $\rho_{m+1}^{M}=\omega$, replacing $M$ with $\mathrm{cHull}_{m+1}^{M}\left(\vec{p}_{m}^{M}\right)$. Now argue as in 2.13 .

\section{The bicephalus \& the cephalanx}

3.1 Definition. An exact bicephalus is a tuple $B=(\rho, M, N)$ such that:

1. $M$ and $N$ are premice.

2. $\rho<\min \left(\mathrm{OR}^{M}, \mathrm{OR}^{N}\right)$ and $\rho$ is a cardinal of both $M$ and $N$.

3. $M\left\|\rho^{+M}=N\right\| \rho^{+N}$.

4. $M$ is $\rho$-sound and for some $m \in\{-1\} \cup \omega$, we have $\rho_{m+1}^{M} \leq \rho$. Likewise for $N$ and $n \in\{-1\} \cup \omega$.

We say $B$ is non-trivial iff $M \neq N$. Write $\rho^{B}=\rho$ and $M^{B}=M$ and $N^{B}=N$, and $m^{B}, n^{B}$ for the least $m, n$ as above. Let $\left(\rho^{+}\right)^{B}$ be $\rho^{+M}=\rho^{+N}$. We say $B$ has degree $\left(m^{B}, n^{B}\right)$. We say that $B$ is sound iff $M$ is $m^{B}+1$-sound and $N$ is $n^{B}+1$-sound.

From now on we just say bicephalus instead of exact bicephalus. In connection with bicephali of degree $(m, n)$ with $\min (m, n)=-1$, we need the following:

3.2 Definition. The terminology/notation (near) (-1)-embedding, $(-1)$ lifting embedding, Ult $-1, \mathfrak{C}_{-1}$, and degree $(-1)$ iterability are defined by replacing ' -1 ' with ' 0 '. For $n>-1$ and appropriate premice $M$, the core embedding $\mathfrak{C}_{n}(M) \rightarrow \mathfrak{C}_{-1}(M)$ is just the core embedding $\mathfrak{C}_{n}(M) \rightarrow \mathfrak{C}_{0}(M) . \quad \dashv$ 
3.3 Definition. Let $q<\omega$. A passive right half-cephalanx of degree $q$ is a tuple $B=(\gamma, \rho, Q)$ such that:

1. $Q$ is a premouse,

2. $\gamma$ is a cardinal of $Q$ and $\gamma^{+Q}=\rho<\mathrm{OR}^{Q}$,

3. $Q$ is $\gamma$-sound,

4. $\rho_{q+1}^{Q} \leq \gamma<\rho_{q}^{Q}$.

An active right half-cephalanx (of degree $q=0)$ is a $B=(\gamma, \rho, Q)$ with:

1. $Q$ is an active segmented-premouse,

2. $\gamma$ is the largest cardinal of $Q$ and $\gamma<\rho=\mathrm{OR}^{Q}$.

A right half-cephalanx $B$ is either a passive, or active, right half-cephalanx. We write $\gamma^{B}, \rho^{B}, Q^{B}, q^{B}$ for $\gamma, \rho, Q, q$ as above. If $B$ is active, we write $S^{B}=$ $R^{B}=\operatorname{Ult}\left(Q, F^{Q}\right)$. If $B$ is passive, we write $S^{B}=Q$.

Note that if $B=(\gamma, \rho, Q)$ is a right-half cephalanx, then $B$ is active iff $Q \mid \rho$ is active. So it might be that $B$ is passive but $Q$ is active with $\rho<\mathrm{OR}^{Q}$.

3.4 Definition. Let $m \in\{-1\} \cup \omega$ and $q<\omega$. A cephalanx of degree $(m, q)$ is a tuple $B=(\gamma, \rho, M, Q)$ such that, letting $B^{\prime}=(\gamma, \rho, Q)$, we have:

1. $(\gamma, \rho, Q)$ is a right-half cephalanx of degree $q$,

2. $M$ is a premouse,

3. $\rho=\gamma^{+M}<\mathrm{OR}^{M}$,

4. $M\left\|\rho^{+M}=S^{B^{\prime}}\right\| \rho^{+M}$,

5. $M$ is $\rho$-sound,

6. $\rho_{m+1}^{M} \leq \rho<\rho_{m}^{M}$.

We say that $B$ is active (passive) iff $B^{\prime}$ is active (passive). ${ }^{15}$ We write $\gamma^{B}, \rho^{B}$, etc, for $\gamma, \rho$, etc. We write $R^{B}$ for $R^{B^{\prime}}$, if it is defined, and $S^{B}$ for $S^{B^{\prime}}$. We say $B$ is exact iff $\left(\rho^{+}\right)^{S^{B}}=\rho^{+M}$, and $B$ is sound iff $M$ is $(m+1)$-sound.

Suppose $B$ is active. Let $R=R^{B}$. We say $B$ is non-trivial iff $M \Varangle R$. If $B$ is non-exact, let $N^{B}$ denote the $N \triangleleft R$ such that $\rho^{+N}=\rho^{+M}$ and $\rho_{\omega}^{N}=\rho$, and let $n^{B}$ denote the $n \in\{-1\} \cup \omega$ such that $\rho_{n+1}^{N}=\rho<\rho_{n}^{N}$.

Now suppose $B$ is passive. We say $B$ is non-trivial iff $M \nsubseteq Q$. Let $N^{B}$ denote the $N \unlhd Q$ such that $\rho^{+N}=\rho^{+M}$ and $\rho_{\omega}^{N} \leq \rho$. Let $n^{B}$ be the $n \in$ $\{-1\} \cup \omega$ such that $\rho_{n+1}^{N^{B}} \leq \rho<\rho_{n}^{N^{B}}$.

A pm-cephalanx is a cephalanx $(\gamma, \rho, M, Q)$ such that $Q$ is a premouse. $\dashv$

\footnotetext{
${ }^{15}$ Note that a passive cephalanx $(\gamma, \rho, M, Q)$ might be such that $M$ and/or $Q$ is/are active.
} 
3.5 Definition. A cephal is either a bicephalus or a cephalanx. Let $B$ be a cephal, and let $M=M^{B}$.

A short extender $E$ is weakly amenable to $B$ iff $\operatorname{cr}(E)<\rho^{B}$ and $E$ is weakly amenable to $M$.

For $\alpha \leq \rho^{B}$, let $B\|\alpha=M\| \alpha$, and for $\alpha<\rho^{B}$, let $B|\alpha=M| \alpha$ and $\left(\alpha^{+}\right)^{B}=$ $\left(\alpha^{+}\right)^{M}$. We write $P \triangleleft B$ iff $P \triangleleft B \| \rho^{B}$. Let $C, \alpha$ be such that $\alpha \leq \rho^{B}$, and either $C$ is a segmented-premouse and $\alpha \leq \mathrm{OR}^{C}$, or $C$ is a cephal and $\alpha \leq \rho^{C}$. Then we write $(B \sim C) \| \alpha$ iff $B\|\alpha=C\| \alpha$. If also $\alpha<\rho^{B}$ and either $C$ is a segmented-premouse or $\alpha<\rho^{C}$, we use the same notation with "|" replacing "|l". We also use similar notation with more than two structures.

A structure with the first order properties of a cephal or other related structures is wellfounded if each of the constituent models are wellfounded. $\dashv$

We will consider ultrapowers and iterates of cephals, and also other related structures, and hence the wellfoundedness of such iterates. Because of the symmetry of bicephali and partial symmetry of cephalanxes, we often state facts for just one side of the symmetry, even when they hold for both. The proofs of the next two lemmas are routine and omitted. In 3.6-3.12 below, the extender $E$ might be long.

3.6 Lemma. Let $Q$ be an active segmented-premouse. Let $E$ be an extender over $Q$ with $\operatorname{ms}(E) \leq \operatorname{cr}\left(F^{Q}\right)+1$. Let $R=\operatorname{Ult}\left(Q, F^{Q}\right)$ and $Q^{\prime}=\operatorname{Ult}\left(Q^{\prime}, E\right)$ and $R^{\prime}=\operatorname{Ult}\left(Q^{\prime}, F^{Q^{\prime}}\right)$. Then $R^{\prime}=\operatorname{Ult}(R, E)$ and the ultrapower embeddings commute. Moreover, $i_{E}^{R}=\psi_{i_{E}^{Q}}$.

3.7 Lemma. Let $Q$ be an active segmented-premouse. Let $E$ be an extender over $Q$ with $\left(\operatorname{cr}\left(F^{Q}\right)^{+}\right)^{Q}<\operatorname{cr}(E)$. Let $R=\operatorname{Ult}\left(Q, F^{Q}\right)$ and $R^{*}=\operatorname{Ult}(R, E)$ and $Q^{\prime}=\operatorname{Ult}(Q, E)$. Then $\operatorname{Ult}\left(Q, F^{Q^{\prime}}\right)=R^{*}$ and the ultrapower embeddings commute. ${ }^{16}$ Let $\psi: R \rightarrow R^{*}$ be given by the Shift Lemma (applied to id : $Q \rightarrow Q$ and $\left.i_{E}^{Q}\right)$. Then $i_{E}^{R}=\psi$.

3.8 Definition. Let $E$ be a (possibly long) extender. For a seg-pm $M, E$ is reasonable for $M$ iff $E$ is over $M$ and either $M$ is passive or letting $\kappa=\operatorname{cr}\left(F^{M}\right)$, $i_{E}^{M}$ is continuous at $\left(\kappa^{+}\right)^{M}$, and if $M \models " \kappa^{++}$exists" then $i_{E}^{M}$ is continuous at $\left(\kappa^{++}\right)^{M}$. For a bicephalus $B=(\rho, M, N), E$ is reasonable for $B$ iff $E$ is over $B \| \rho$, if $m^{B} \leq 0$ then $E$ is reasonable for $M$, and if $n^{B} \leq 0$ then $E$ is reasonable for $N$. For a cephalanx $B=(\gamma, \rho, M, Q), E$ is reasonable for $B$ iff $E$ is over $B \| \rho,{ }^{17}$ if $q^{B} \leq 0$ then $E$ is reasonable for $Q$, if $m^{B} \leq 0$ then $E$ is reasonable for $M$, and if $N^{B}$ is defined and $n^{B} \leq 0$ then $E$ is reasonable for $N^{B}$.

3.9 Lemma. Let $Q$ be an active seg-pm, $E$ reasonable for $Q$. Let $Q^{\prime}=$ $\operatorname{Ult}(Q, E), R=\operatorname{Ult}\left(Q, F^{Q}\right), R^{\prime}=\operatorname{Ult}\left(Q^{\prime}, F^{Q^{\prime}}\right), R^{*}=\operatorname{Ult}(R, E)$. Let $\kappa=$ $\operatorname{cr}\left(F^{Q}\right)$ and $\eta=\left(\kappa^{++}\right)^{Q}$. Further:

\footnotetext{
${ }^{16}$ Note that in the conclusion, it is $\operatorname{Ult}\left(Q, F^{Q^{\prime}}\right)$, not $\operatorname{Ult}\left(Q^{\prime}, F^{Q^{\prime}}\right)$.

${ }^{17}$ Hence $E$ is equivalent to an extender $E^{\prime}$ with $\operatorname{ms}\left(E^{\prime}\right) \leq \gamma+1$.
} 


$$
\begin{aligned}
& \text { - If } \eta<\mathrm{OR}^{Q} \text {, let } \gamma=i_{Q R}(\eta), \gamma^{*}=i_{E}^{R}(\gamma), \eta^{\prime}=i_{E}^{Q}(\eta) . \text { Then } \gamma^{*}=i_{Q^{\prime} R^{\prime}}\left(\eta^{\prime}\right) . \\
& \text { - If } \eta=\mathrm{OR}^{Q} \text {, let } \gamma=\mathrm{OR}^{R}, \gamma^{*}=\mathrm{OR}^{R^{*}} \text { and } \eta^{\prime}=\mathrm{OR}^{Q^{\prime}} .
\end{aligned}
$$

Then in either case, $\left(R^{*} \sim R^{\prime}\right) \mid \gamma^{*}$ and

$$
i_{E}^{R} \circ i_{Q R} \uparrow(Q \mid \eta)=i_{Q^{\prime} R^{\prime}} \circ i_{E}^{Q} \uparrow(Q \mid \eta) .
$$

Moreover, let $\psi: R\left|\gamma \rightarrow R^{\prime}\right| \gamma^{\prime}$ be induced by the Shift Lemma with $i_{Q Q^{\prime}} \uparrow(Q \mid \eta)$ and $i_{Q Q^{\prime}}$. Then $\psi=i_{E}^{R} \uparrow(R \mid \gamma)$.

Proof. Let $G$ be the length $i_{E}(\kappa)$ extender derived from $E$. Let $j: \operatorname{Ult}(Q, G) \rightarrow$ $\operatorname{Ult}(Q, E)$ be the factor embedding. Then $\operatorname{cr}(j)>\left(i_{G}^{Q}(\kappa)^{++}\right)^{U_{G}}$ since $E$ is reasonable. Apply 3.6 to $G$, and then 3.7 to the extender derived from $j$.

3.10 Definition. Let $M$ be a type 3 premouse. The expansion of $M$ is the active segmented-premouse $M_{*}$ such that $M_{*}\left|\operatorname{cr}\left(F^{M_{*}}\right)=M\right| \operatorname{cr}\left(F^{M}\right)$, and $F^{M_{*}}$ is the Jensen-indexed version of $F^{M}$. That is, let $F=F^{M}$, let $\mu=\operatorname{cr}(F)$, let $\gamma=\left(\mu^{+}\right)^{M}$, let $\gamma^{\prime}=i_{F}(\gamma)$, let $R=\operatorname{Ult}\left(M, F^{M}\right)$; then $M_{*}|| \operatorname{OR}\left(M_{*}\right)=R \mid \gamma^{\prime}$, and $F^{M_{*}}$ is the length $i_{F}(\mu)$ extender derived from $i_{F}$.

Combining $[3, \S 9]$ with a simple variant of 3.9 one gets:

3.11 Fact. Let $Q$ be a type 3 premouse. Let $E$ be an extender over $Q^{\text {sq }}$, reasonable for $Q$. Let $Q_{*}$ be the expansion of $Q$, let $U_{*}=\operatorname{Ult}\left(Q_{*}, E\right)$ and $U=\operatorname{Ult}_{0}(Q, E)$. Suppose $U_{*}$ is wellfounded. Then $U$ is wellfounded and $U_{*}$ is its expansion. Let $i_{*}: Q_{*} \rightarrow U_{*}$ and $i_{0}: Q \rightarrow U$ be the ultrapower maps (literally, $\operatorname{dom}\left(i_{*}\right)=Q_{*}$ and $\left.\operatorname{dom}\left(i_{0}\right)=Q^{\mathrm{sq}}\right)$. Then $i_{0}=i_{*}\left\lceil Q^{\mathrm{sq}}\right.$, and $i_{*}=\psi_{i_{0}}\left\lceil Q_{*}\right.$.

3.12 Remark. We will apply 3.9 and 3.11 when $E$ is the extender of iteration map $i_{\alpha \beta}^{\mathcal{T}}$ or $i_{\alpha \beta}^{* \mathcal{T}}$, where $(\alpha, \beta]_{\mathcal{T}}$ does not drop and $\operatorname{deg}^{\mathcal{T}}(\alpha)=0$.

3.13 Definition (Ultrapowers of bicephali). Let $B=(\rho, M, N)$ be a bicephalus of degree $(m, n)$ and $E$ an extender reasonable for $B$. We have the ultrapower map $i=i_{E}^{M, m}: M \rightarrow \operatorname{Ult}_{m}(M, E)$, and $j=i_{E}^{N, n}$. Let $\rho^{\prime}=\sup i " \rho=\sup j$ " $\rho$ and define

$$
\operatorname{Ult}(B, E)=\left(\rho^{\prime}, \operatorname{Ult}_{m}(M, E), \operatorname{Ult}_{n}(N, E)\right) .
$$

3.14 Definition. Let $B$ be a bicephalus. The associated augmented bicephalus is the tuple $B_{*}=\left(\rho, M, N, M_{*}, N_{*}\right)$ where if $m \geq 0$ then $M_{*}=M$, and otherwise $M_{*}$ is the expansion of $M$; likewise for $N_{*}$. (Note that if $m=-1$ then $M$ is type 3 and $\rho=\nu\left(F^{M}\right)$.) Let $E$ reasonable for $B$. If $m \geq 0$ let $\widetilde{M}=\operatorname{Ult}_{m}(M, E)$; otherwise let $\widetilde{M}=\operatorname{Ult}\left(M_{*}, E\right)$. Likewise for $\widetilde{N}$. We define $\operatorname{Ult}\left(B_{*}, E\right)=\operatorname{Ult}(B, E)^{\wedge}\langle\widetilde{M}, \widetilde{N}\rangle$.

3.15 Lemma. Let $B=(\rho, M, N)$ be a bicephalus of degree $(m, n), B_{*}=$ $\left(\rho, M, N, M_{*}, N_{*}\right), E$ reasonable for $B, U=\operatorname{Ult}(B, E)$ and $\widetilde{U}=\operatorname{Ult}\left(B_{*}, E\right)=$ $\left(\rho^{U}, M^{U}, N^{U}, \widetilde{M}, \widetilde{N}\right)$. Let $i=i_{E}^{M, m}$ and $j=j_{E}^{N, n}$. If $m \geq 0$ let $i_{*}=i$; otherwise let $i_{*}: M_{*} \rightarrow \widetilde{M}$ be the ultrapower map. Likewise $j_{*}$. If $\widetilde{U}$ is wellfounded then: 
(1) $U$ is a bicephalus of degree $(m, n)$ and $\widetilde{U}=U_{*}$.

(2) $U$ is trivial iff $B$ is trivial.

(3) $i\left(p_{m+1}^{M} \backslash \rho\right)=p_{m+1}^{M^{U}} \backslash \rho^{U}$.

(4) $i_{*} \uparrow\left(\rho^{+}\right)^{B}=j_{*} \uparrow\left(\rho^{+}\right)^{B}$ and $i_{*}, j_{*}$ are continuous/cofinal at $\left(\rho^{+}\right)^{B} \cdot .^{18}$

(5) $i_{*}=\psi_{i} \uparrow M_{*}$.

(6) Suppose $E$ is short and weakly amenable to B. Then $M^{U}$ is $(m+1)$-sound iff $M$ is $(m+1)$-sound and $\operatorname{cr}(E)<\rho_{m+1}^{M}$. If $M^{U}$ is $(m+1)$-sound then $\rho_{m+1}^{M^{U}}=\sup i " \rho_{m+1}^{M}$ and $p_{m+1}^{M^{U}}=i\left(p_{m+1}^{M}\right)$.

Likewise regarding $N, n, E$.

Proof. Part (6) is by [12, 2.20], (3) is a standard calculation using generalized solidity witnesses (see [18]), and (5) is by $3.11((5)$ is trivial when $m \geq 0)$.

Part (4): Let $W=\operatorname{Ult}\left(B \|\left(\rho^{+}\right)^{B}, E\right)$ and $k: B \|\left(\rho^{+}\right)^{B} \rightarrow W$ the ultrapower map and $\widetilde{\rho}=k(\rho)$. We claim that $(\dagger): k=i_{*} \uparrow\left(\rho^{+}\right)^{B}$ and $\widetilde{M} \|\left(\widetilde{\rho}^{+}\right)^{\widetilde{M}}=W$.

If $m \leq 0$ this is immediate. If $m>0$, then because $\left(\rho^{+}\right)^{B} \leq \rho_{m}^{M}$, by $[3, \S 6]$, all functions forming the ultrapower $M^{U}$ with codomain $\left(\rho^{+}\right)^{B}$ are in fact in $B \|\left(\rho^{+}\right)^{B}$, which gives $(\dagger)$. Now (4) follows from $(\dagger)$.

Part (1): By 3.11, $\widetilde{M}$ is the expansion of $M^{U}$. We have $\rho^{U} \leq \widetilde{\rho}$ and by $(\dagger)$, $\widetilde{M}\left\|\left(\widetilde{\rho}^{+}\right)^{\widetilde{M}}=\widetilde{N}\right\|\left(\widetilde{\rho}^{+}\right)^{\widetilde{N}}$. If $m \geq 0$ then $\widetilde{\rho}<\rho_{m}\left(M^{U}\right)$. The rest of (1) is routine.

Part (2): Assume $M \neq N$ but $m=n$. By $\rho$-soundness, there is an $\mathrm{r} \Sigma_{m+1}$ formula $\varphi$ and $\alpha<\rho$ with

$$
M \models \varphi\left(p_{m+1}^{M} \backslash \rho, \vec{p}_{m}^{M}, \alpha\right) \quad \Longleftrightarrow \quad N \models \neg \varphi\left(p_{m+1}^{N} \backslash \rho, \vec{p}_{m}^{N}, \alpha\right) .
$$

Now $i, j$ are $\mathrm{r} \Sigma_{m+1}$-elementary, and by $(\dagger), i(\alpha)=j(\alpha)$; let $\alpha^{\prime}=i(\alpha)$. So by (3), we get $M^{U} \neq N^{U}$ and in fact

$$
M^{U} \models \varphi\left(p_{m+1}^{M^{U}} \backslash \rho^{U}, \vec{p}_{m}^{M^{U}}, \alpha^{\prime}\right) \quad \Longleftrightarrow \quad N^{U} \models \neg \varphi\left(p_{m+1}^{N^{U}} \backslash \rho^{U}, \vec{p}_{m}^{N^{U}}, \alpha^{\prime}\right) .
$$

3.16 Definition (Ultrapowers of cephalanxes). Let $B=(\gamma, \rho, M, Q)$ be a cephalanx of degree $(m, q)$ and let $E$ be reasonable for $B$. Let $M^{\prime}=\operatorname{Ult}_{m}(M, E)$, $\gamma^{\prime}=i_{E}^{M, m}(\gamma)$ and $\rho^{\prime}=\sup i_{E}^{M, m}$ " $\rho$. If $B$ is active let $Q^{\prime}=\operatorname{Ult}(Q, E)$ (recall the ultrapower $\operatorname{Ult}(Q, E)$ is simple; it might be that $Q$ is type 3 , and we could have $\operatorname{cr}(E)=\gamma$.) If $B$ is passive let $Q^{\prime}=\operatorname{Ult}_{q}(Q, E)$. Then we define $\operatorname{Ult}(B, E)=\left(\gamma^{\prime}, \rho^{\prime}, M^{\prime}, Q^{\prime}\right)$.

3.17 Lemma. In the context of 3.16, suppose that $B$ is passive, and that $U=$ $\operatorname{Ult}(B, E)$ is wellfounded. Let $i=i_{E}^{M, m}$ and $j=i_{E}^{Q}$. Then:

\footnotetext{
${ }^{18}$ That is, if $\left(\rho^{+}\right)^{B} \in \operatorname{dom}\left(i_{*}\right)$ then $i_{*}$ is continuous there; if $m \geq 0$ and $\left(\rho^{+}\right)^{B}=\rho_{0}^{M}$ then $\rho_{0}^{M^{U}}=\sup i^{\text {“ }}\left(\rho^{+}\right)^{B}$; if $m=-1$ and $\left(\rho^{+}\right)^{B}=\operatorname{OR}\left(M_{*}\right)$ then $\operatorname{OR}\left(\left(M^{U}\right)_{*}\right)=\sup i_{*}$ " $\left(\rho^{+}\right)^{B}$.
} 
(1) $U$ is a passive cephalanx of degree $(m, q)$.

(2) $i \uparrow \rho=j\lceil\rho$.

(3) If $\rho \in \mathfrak{C}_{0}(M)$ then $\rho^{\prime}=i(\rho)$; otherwise $\rho^{\prime}=\rho_{0}\left(M^{U}\right)$. Likewise $Q, j, Q^{U}$.

(4) $\psi_{i}(\rho)=\psi_{j}(\rho)=\rho^{\prime}$.

(5) If $\rho^{+M} \in \operatorname{dom}\left(\psi_{i}\right)$ then $\psi_{i}$ is continuous at $\rho^{+M}$; otherwise $M$ is passive, $\mathrm{OR}^{M}=\rho^{+M}$ and $\mathrm{OR}\left(M^{U}\right)=\sup i^{\prime \prime} \mathrm{OR}^{M}$.

(6) $\psi_{i} \uparrow \rho^{+M}=\psi_{j} \uparrow \rho^{+M}$.

(7) $i\left(p_{m+1}^{M} \backslash \rho\right)=p_{m+1}^{M^{U}} \backslash \rho^{\prime}$.

(8) Suppose $E$ is short and weakly amenable to B. Then $M^{U}$ is $(m+1)$-sound iff $M$ is $(m+1)$-sound and $\operatorname{cr}(E)<\rho_{m+1}^{M}$. If $M^{U}$ is $(m+1)$-sound then $\rho_{m+1}^{M^{U}}=\sup i " \rho_{m+1}^{M}$ and $p_{m+1}^{M^{U}}=i\left(p_{m+1}^{M}\right)$.

(9) If $B$ is non-exact then $U$ is non-exact.

(10) If $B$ is exact ( so $N^{B}=Q$ ) but $U$ is not, then $0 \leq n^{B}<q$.

(11) Suppose that $B$ is non-trivial and that suitable condensation holds below $(Q, q)$. Let $N=N^{B}$ and $n=n^{B}$. Then:

(i) $U$ is non-trivial,

(ii) $N^{U}=\operatorname{Ult}_{n^{B}}(N, E)$ and $n^{U}=n$,

(iii) Parts (2)-(8) hold with ' $M$ ' replaced by ' $N$ ' and ' $m$ ' by ' $n$ '.

We also have $j\left(p_{q+1}^{Q} \backslash \gamma\right)=p_{q+1}^{Q^{U}} \backslash \gamma^{U}$, but we won't need this.

Proof. Parts (2)-(8) are much as in 3.15. (For (6), note that for $A \in \mathcal{P}(\rho) \cap M$, the value of $\psi_{i}(A)$ is determined by the values of $\psi_{i}(A \cap \alpha)$ for $\alpha<\rho$; likewise for $\psi_{j}(A)$.) So (1) follows. Part (9) follows from (5) and (6); part (10) is easy.

Part (11): Consider the case that $B$ is exact. Part (iii) is as for $M$, so consider (i) and (ii). As $B$ is exact, $N=Q$. By the proof of 3.15 , we have $\operatorname{Ult}_{n}(Q, E) \neq \operatorname{Ult}_{m}(M, E)$, so it suffices to see that

$$
U_{n}=\operatorname{Ult}_{n}(Q, E) \unlhd Q^{U}=\operatorname{Ult}_{q}(Q, E)=U_{q},
$$

so assume $n<q$. If $n=-1$ then $U_{n}=U_{q}$, so assume $n \geq 0$, so $\rho \in \mathfrak{C}_{0}(Q)$ and

$$
\rho_{q+1}^{Q} \leq \gamma<\rho=\rho_{q}^{Q}=\rho_{n+1}^{Q}<\rho_{n}^{Q} .
$$

Let $\sigma: U_{n} \rightarrow U_{q}$ be the natural factor map. Let $j_{n}: Q \rightarrow U_{n}$ and $j_{q}: Q \rightarrow U_{q}$ be the ultrapower maps. Then $\sigma \circ j_{n}=j_{q}, \sigma$ is $\vec{p}_{n+1}$-preserving $n$-lifting and $\operatorname{cr}(\sigma)>\rho^{\prime}$. Also, $U_{n}, U_{q}$ are $(n+1)$-sound and $\rho_{n+1}^{U_{n}}=\rho^{\prime}=\rho_{n+1}^{U_{q}}$.

Suppose $\left(\left(\rho^{\prime}\right)^{+}\right)^{U_{n}}=\left(\left(\rho^{\prime}\right)^{+}\right)^{U_{q}}<\operatorname{cr}(\sigma)$. Then $\rho_{n}^{U_{q}}=\sup \sigma^{\prime \prime} \rho_{n}^{U_{n}}$, since otherwise, using the previous paragraph and as in the proof of 2.4 , we get 
$U_{n} \in U_{q}$, collapsing $\left(\left(\rho^{\prime}\right)^{+}\right)^{U_{q}}$ in $U_{q}$. So by 2.4, $\sigma$ is an $n$-embedding. But $\rho_{n+1}^{U_{q}} \cup p_{n+1}^{U_{q}} \subseteq \operatorname{rg}(\sigma)$, so then $U_{n}=U_{q}$, which suffices.

Now suppose $\left(\left(\rho^{\prime}\right)^{+}\right)^{U_{n}}<\left(\left(\rho^{\prime}\right)^{+}\right)^{U_{q}}$. Then much as before, $\rho_{n}^{U_{q}}>\sup \sigma^{\prime \prime} \rho_{n}^{U_{n}}$. Let $\sigma^{*}=\sigma \uparrow\left(U_{n} \| \rho_{n}^{U_{n}}\right)$. By 2.4 we get $U_{n}, \sigma^{*} \in U_{q}$ and $\left(U_{n}, \sigma^{*}\right)$ is $n$-suitable for $U_{q}$. By suitable condensation below $(Q, q)$ and 2.11 and since $U_{q} \mid \rho^{\prime}$ is passive, therefore $U_{n} \triangleleft U_{q}$, which suffices.

If $B$ is non-exact, so $N \triangleleft Q$, let $U_{n}=\operatorname{Ult}_{n}(N, E)$, consider the factor embedding $\sigma: U_{n} \rightarrow j(N)$ and show $U_{n} \unlhd j(N)$. This completes the proof.

3.18 Lemma. In the context of 3.16, suppose that $B$ is active, and that $U=$ $\operatorname{Ult}(B, E)$ and $R^{U}$ are wellfounded. Let $i=i_{E}^{M, m}$ and $j=i_{E}^{Q}$. Then:

(1) $U$ is an active cephalanx of degree $(m, 0)$.

(2) If $\rho \in \mathfrak{C}_{0}(M)$ then $\rho^{\prime}=i(\rho)$; otherwise $\rho^{\prime}=\rho_{0}\left(M^{U}\right)$.

(3) 3.17(2) and 3.17(4)-(8) hold.

(4) $U$ is exact iff $B$ is exact.

(5) Suppose that $B$ is non-exact and non-trivial and that suitable condensation holds below $(Q, 0)$. Let $N=N^{B}$ and $n=n^{B}$. Then:

(i) $U$ is non-trivial,

(ii) $N^{U}=\operatorname{Ult}_{n^{B}}(N, E)$ and $n^{U}=n$,

(iii) Parts (2)-(3) hold with ' $M$ ' replaced by ' $N$ ' and ' $m$ ' by ' $n$ '.

Proof. This follows from 3.9, 3.11 and the proof of $3.17 .{ }^{19}$

3.19 Lemma. Let $C$ be a degree $(m, k)$ cephal. If $C$ is a bicephalus let $B=C_{*}$; otherwise let $B=C$. Let $\left\langle E_{\alpha}\right\rangle_{\alpha<\lambda}$ be a sequence of short extenders. Let $B_{0}=B$, $B_{\alpha+1}=\operatorname{Ult}\left(B_{\alpha}, E_{\alpha}\right)$, and $B_{\gamma}$ be the direct limit at limit $\gamma$. Suppose for each $\alpha \leq \lambda, B_{\alpha}$ is wellfounded and if $\alpha<\lambda$ then $E_{\alpha}$ is weakly amenable to $B_{\alpha}$.

If $C$ is a bicephalus (passive cephalanx, active cephalanx, respectively) then the conclusions of 3.15 (3.17, 3.18, respectively) apply to $B$ and $B_{\lambda}$, together with the associated iteration maps, after deleting the sentence "Suppose $E$ is short and weakly amenable to B." and replacing the phrase $\operatorname{cr}(E)<\rho_{m+1}^{M}$ " with "cr $\left(E_{\alpha}\right)<\rho_{m+1}^{M_{\alpha}}$ for each $\alpha<\gamma$ ".

Proof. If $C$ is a bicephalus, this mostly follows from 3.15, [12, 2.20] and 3.11 by induction. At limit stages, use [12, 2.20] directly to prove 3.15(6). To see $3.15(4)$, replace the iteration used to define $C_{\gamma}$ with a single (possibly long) extender $E$, and apply 3.15 . The cephalanx cases are similar.

\footnotetext{
${ }^{19}$ In 3.9 we set $\eta=\left(\kappa^{++}\right)^{Q}$, and the reader might wonder why we didn't just use $\eta=\left(\kappa^{+}\right)^{Q}$. We need the larger value here if $Q$ has superstrong type.
} 
3.20 Definition (Iteration trees on bicephali). Let $B=(\rho, M, N)$ be a bicephalus of degree $(m, n)$ and let $\eta \in \mathrm{OR} \backslash\{0\}$. An iteration tree on $B$, of length $\eta$, is a pair $\mathcal{T}=\left(<_{\mathcal{T}},\left\langle E_{\alpha}\right\rangle_{\alpha+1<\eta}\right)$ such that there are sequences

$$
\left\langle B_{\alpha}, M_{\alpha}, N_{\alpha}\right\rangle_{\alpha<\eta} \text { and }\left\langle B_{\alpha+1}^{*}, M_{\alpha+1}^{*}, N_{\alpha+1}^{*}\right\rangle_{\alpha+1<\eta},
$$

of models, sequences of embeddings

$$
\left\langle i_{\alpha \beta}, j_{\alpha \beta}\right\rangle_{\alpha, \beta<\eta} \text { and }\left\langle i_{\alpha+1}^{*}, j_{\alpha+1}^{*}\right\rangle_{\alpha+1<\eta},
$$

sequences of ordinals $\left\langle\rho_{\alpha}\right\rangle_{\alpha<\eta}$ and $\left\langle\mathrm{cr}_{\alpha}, \nu_{\alpha}, \mathrm{lh}_{\alpha}, \rho_{\alpha+1}^{*}\right\rangle_{\alpha+1<\eta}$, sets $\mathscr{B}, \mathscr{M}, \mathscr{N} \subseteq \eta$ (specifying types and origins of structures), a function "deg" with domain $\eta$ (specifying degrees), and a set $\mathscr{D} \subseteq \eta$ (specifying drops in model), such that:

1. $<_{\mathcal{T}}$ is an iteration tree order on $\eta$, with the usual properties.

2. $B_{0}=\left(\rho_{0}, M_{0}, N_{0}\right)=B$ and $\operatorname{deg}(0)=(m, n)$ and $i_{00}=$ id and $j_{00}=$ id.

3. $\mathscr{B}, \mathscr{M}, \mathscr{N}$ are disjoint and for each $\alpha<\eta$, either

(a) $\alpha \in \mathscr{B}$ and $B_{\alpha}=\left(\rho_{\alpha}, M_{\alpha}, N_{\alpha}\right)$ is a bicephalus of degree $(m, n)=$ $\operatorname{deg}(\alpha)$, or

(b) $\alpha \in \mathscr{M}$ and $B_{\alpha}=M_{\alpha}$ is a segmented-premouse and $N_{\alpha}=\emptyset$, or

(c) $\alpha \in \mathscr{N}$ and $B_{\alpha}=N_{\alpha}$ is a segmented-premouse and $M_{\alpha}=\emptyset$.

4. For each $\alpha+1<\eta$ :

(i) Either $E_{\alpha} \in \mathbb{E}_{+}\left(M_{\alpha}\right)$ or $E_{\alpha} \in \mathbb{E}_{+}\left(N_{\alpha}\right)$.

(ii) $\mathrm{cr}_{\alpha}=\operatorname{cr}\left(E_{\alpha}\right)$ and $\nu_{\alpha}=\nu\left(E_{\alpha}\right)$ and $\operatorname{lh}_{\alpha}=\operatorname{lh}\left(E_{\alpha}\right)$.

(iii) For all $\beta<\alpha$ we have $\operatorname{lh}_{\beta} \leq \operatorname{lh}_{\alpha}$.

(iv) $\operatorname{pred}^{\mathcal{T}}(\alpha+1)$ is the least $\beta$ such that $\operatorname{cr}_{\alpha}<\nu_{\beta}$.

Fix $\alpha+1<\eta$ and $\beta=\operatorname{pred}^{\mathcal{T}}(\alpha+1)$ and $\kappa=\mathrm{cr}_{\alpha}$.

5. Suppose $\beta \in \mathscr{B}$ and $\kappa<\rho_{\beta}$ and $E_{\alpha}$ is total over $B_{\beta} \| \rho_{\beta}$. Then $\operatorname{deg}(\alpha+1)=$ $(m, n)$ and

$$
\left(\rho_{\alpha+1}^{*}, M_{\alpha+1}^{*}, N_{\alpha+1}^{*}\right)=B_{\alpha+1}^{*}=B_{\beta} \text { and } B_{\alpha+1}=\operatorname{Ult}\left(B_{\alpha+1}^{*}, E\right),
$$

$i_{\alpha+1}^{*}: M_{\alpha+1}^{*} \rightarrow M_{\alpha+1}$ is the ultrapower map, $i_{\alpha+1, \alpha+1}=\mathrm{id}$ and

$$
i_{\gamma, \alpha+1}=i_{\alpha+1}^{*} \circ i_{\gamma \beta}: M_{\gamma} \rightarrow M_{\alpha+1}
$$

for $\gamma \leq_{\mathcal{T}} \beta$; likewise for $j_{\alpha+1}^{*}$ etc. 
6. Suppose that $E_{\beta} \in \mathbb{E}_{+}\left(M_{\beta}\right)$. Suppose that either $\beta \notin \mathscr{B}$ (so $\beta \in \mathscr{M}$ ), or $\kappa<\rho_{\beta}$ and $E_{\alpha}$ is not total over $B_{\beta} \| \rho_{\beta}$. Then we put $\alpha+1 \in \mathscr{M}$, $N_{\alpha+1}=N_{\alpha+1}^{*}=\emptyset$, and $j_{\alpha+1}^{*}$, etc, are undefined. We set $M_{\alpha+1}^{*} \unlhd M_{\beta}$ and $\operatorname{deg}(\alpha+1)$, etc, in the manner for degree-maximal trees. Let $k=$ $\operatorname{deg}(\alpha+1)$. Then

$$
M_{\alpha+1}=\operatorname{Ult}_{k}\left(M_{\alpha+1}^{*}, E_{\alpha}\right)
$$

and $i_{\alpha+1}^{*}$, etc, are defined in the usual manner. We set $B_{\alpha+1}^{*}=M_{\alpha+1}^{*}$ and $B_{\alpha+1}=M_{\alpha+1}$.

7. Suppose that $E_{\beta} \notin \mathbb{E}_{+}\left(M_{\beta}\right)$ (so $\left.E_{\beta} \in \mathbb{E}_{+}\left(N_{\beta}\right)\right)$ and $B_{\alpha+1}$ is not defined through clause 5 . Then we proceed symmetrically to clause 6 (interchanging " $M$ " with " $N$ ").

8. $\alpha+1 \in \mathscr{D}$ iff either $\emptyset \neq M_{\alpha+1}^{*} \triangleleft M_{\beta}$ or $\emptyset \neq N_{\alpha+1}^{*} \triangleleft N_{\beta}$.

9. For every limit $\lambda<\eta, \mathscr{D} \cap[0, \lambda)_{\mathcal{T}}$ is finite, and $\lambda \in \mathscr{B}$ iff $[0, \lambda)_{\mathcal{T}} \subseteq \mathscr{B}$; the models $M_{\lambda}$, etc, and embeddings $i_{\alpha, \lambda}$, etc, are defined via direct limits, and $\operatorname{deg}(\lambda)$ is the common value of $\operatorname{deg}(\alpha)$ for all sufficiently large $\alpha<\mathcal{T} \lambda$.

For $\alpha<\operatorname{lh}(\mathcal{T}), \mathscr{B}(\alpha)$ denotes $\max \left(\mathscr{B} \cap[0, \alpha]_{\mathcal{T}}\right)$.

3.21 Lemma. Let $\mathcal{T}$ be an iteration tree on a bicephalus of degree $(m, n)$ and let $\alpha<\operatorname{lh}(\mathcal{T})$. We write $B_{\alpha}=B_{\alpha}^{\mathcal{T}}$, etc. Then:

1. If $\alpha+1<\operatorname{lh}(\mathcal{T})$ then $E_{\alpha}$ is weakly amenable to $B_{\alpha+1}^{*}$.

2. If $\alpha+1<\operatorname{lh}(\mathcal{T})$ and $\alpha+1 \notin \mathscr{B}$ then $E_{\alpha}$ is close to $B_{\alpha+1}^{*}$.

3. $\mathscr{B}$ is closed downward under $<_{\mathcal{T}}$ and if $\alpha \in \mathscr{M}$ then $\mathscr{N} \cap[0, \alpha]_{\mathcal{T}}=\emptyset$.

4. If $\alpha \in \mathscr{M}$ and $[0, \alpha]_{\mathcal{T}} \cap \mathscr{D}=\emptyset$ then $m \geq 0$.

5. If $\alpha \in \mathscr{M},[0, \alpha]_{\mathcal{T}} \cap \mathscr{D}=\emptyset, \operatorname{deg}(\alpha)=m$ and $\beta=\mathscr{B}(\alpha)$ then:

- $M_{\beta}$ is $\rho_{\beta}$-sound, whereas $M_{\alpha}$ is $\rho_{\beta}$-solid but not $\rho_{\beta}$-sound,

- $M_{\beta}$ is the $\rho_{\beta}$-core of $M_{\alpha}$ and $i_{\beta \alpha}$ is the $\rho_{\beta}$-core embedding,

$-\rho_{m+1}\left(M_{\beta}\right)=\rho_{m+1}\left(M_{\alpha}\right)$,

- $i_{\beta \alpha}\left(p_{m+1}^{M_{\beta}} \backslash \rho_{\beta}\right)=p_{m+1}^{M_{\alpha}} \backslash \rho_{\beta}$.

6. Suppose $\alpha \in \mathscr{M}$ and $[0, \alpha]_{\mathcal{T}}$ drops in model or degree. Let $k=\operatorname{deg}^{\mathcal{T}}(\alpha)$. Then the core embedding $\mathfrak{C}_{k+1}\left(M_{\alpha}\right) \rightarrow M_{\alpha}$ relates to $\mathcal{T}$ in the manner usual for degree-maximal iteration trees.

Proof. Parts 1, 3 and 4 are easy. For part 2, use essentially the proof of [3, 6.1.5], combined with the following simple observation. Let $\xi+1<\operatorname{lh}(\mathcal{T})$ be such that $[0, \xi]_{\mathcal{T}}$ does not drop in model and $E_{\xi}=F\left(M_{\xi}\right)$. Let $\chi=\operatorname{pred}^{\mathcal{T}}(\xi+1)$. Then $[0, \xi+1]_{\mathcal{T}}$ does not drop in model and $\chi$ is the least $\chi^{\prime} \in[0, \xi]_{\mathcal{T}}$ such that $\operatorname{cr}\left(F\left(M_{\chi^{\prime}}\right)\right)=\mathrm{cr}_{\xi}$. We omit further details of the proof of part 2 .

Parts 5 and 6 now follow as usual. 
3.22 Definition (Iteration trees on cephalanxes). Let $B$ be a cephalanx. The notion of an iteration tree $\mathcal{T}$ on $B$ is defined much as in 3.20. The key differences are as follows. The models of the tree are all either cephalanxes or segmented-premice ${ }^{20}$, and if $B$ is passive, then the models are all either cephalanxes or premice. We write $\left(M_{\alpha}, i_{\alpha \beta}\right)$ and $\left(Q_{\alpha}, k_{\alpha \beta}\right)$, etc, for the models and embeddings above $M^{B}$ and $Q^{B}$ respectively. We write $B_{\alpha}=\left(\gamma_{\alpha}, \rho_{\alpha}, M_{\alpha}, Q_{\alpha}\right)$ when $B_{\alpha}$ is a cephalanx, and otherwise $B_{\alpha}=M_{\alpha} \neq \emptyset$ or $B_{\alpha}=Q_{\alpha} \neq \emptyset$, and write $\mathscr{Q}$ for the set of $\alpha$ such that $B_{\alpha}=Q_{\alpha}$. Let $\iota_{\alpha}=\iota\left(E_{\alpha}\right)$. Other notation is as in 3.20 .

Let $\alpha+1<\ln (\mathcal{T})$. Then:

- Either $E_{\alpha} \in \mathbb{E}_{+}\left(M_{\alpha}\right)$ or $E_{\alpha} \in \mathbb{E}_{+}\left(Q_{\alpha}\right)$.

Let $\kappa=\mathrm{cr}_{\alpha}$. Then:

$-\operatorname{pred}^{\mathcal{T}}(\alpha+1)$ is the least $\beta$ such that $\kappa<\iota_{\beta}$.

Suppose $\beta \in \mathscr{B}$. Then:

- If $E_{\beta} \in \mathbb{E}_{+}\left(M_{\beta}\right)$ and either $\rho_{\beta}<\kappa$ or $E_{\alpha}$ is not total over $M_{\beta}$ then $\alpha+1 \in \mathscr{M}$ and $M_{\alpha+1}^{*} \unlhd M_{\beta}$ and $Q_{\alpha+1}=\emptyset$.

- If $E_{\beta} \notin \mathbb{E}_{+}\left(M_{\beta}\right)$ and either $\rho_{\beta}<\kappa$ or $E_{\alpha}$ is not total over $Q_{\beta}$ then $\alpha+1 \in \mathscr{Q}$ and $Q_{\alpha+1}^{*} \unlhd Q_{\beta}$ and $M_{\alpha+1}=\emptyset$.

Now suppose that $\kappa<\rho_{\beta}$ and $E_{\alpha}$ is total over $B_{\beta} \| \rho_{\beta}\left(\right.$ so $\left.\kappa \leq \gamma_{\beta}\right)$. Then:

- Suppose either $\kappa<\gamma_{\beta}$ or $E_{\beta} \in \mathbb{E}_{+}\left(M_{\beta}\right)$. Then $B_{\alpha+1}^{*}=B_{\beta}{ }^{21}$

- If $\kappa=\gamma_{\beta}$ and $E_{\beta} \notin \mathbb{E}_{+}\left(M_{\beta}\right)^{22}$ then $M_{\alpha+1}=\emptyset$ and $Q_{\alpha+1}^{*}=Q_{\beta}{ }^{23}$

The remaining details are like in 3.20 .

3.23 Lemma. Let $\mathcal{T}$ be an iteration tree on a cephalanx $B=(\gamma, \rho, M, Q)$ of degree $(m, q)$ and let $\alpha+1<\operatorname{lh}(\mathcal{T})$. Then parts $1-6$ of 3.21, replacing ' $\mathscr{N}$ ' with 'Q', hold. Parts 3-6, replacing ' $\mathscr{M}$ ' with ' $\mathscr{Q}$ ', ' $M$ ' with ' $Q$ ', ' $m$ ' with ' $q$ ', ' $\rho$ ' with ' $\gamma$ ', and ' $\mathscr{N}$ ' with ' $\mathscr{M}$ ', hold.

\footnotetext{
${ }^{20}$ In fact, for the cephalanxes $B$ we will produce - in the proof of 5.2) - the models of all trees on $B$ will be either cephalanxes or premice.

${ }^{21}$ Here if $\kappa=\gamma_{\beta}$ (so $E_{\beta} \in \mathbb{E}_{+}\left(M_{\beta}\right)$ ), one might wonder why we do not just set $M_{\alpha+1}^{*}=\emptyset$ and $Q_{\alpha+1}^{*}=Q_{\beta}$. This might be made to work, but doing this, it seems that $E_{\alpha}$ might not be close to $Q_{\alpha+1}^{*}$.

${ }^{22}$ When this situation arises with one of the active cephalanxes we will produce, $Q$ and $Q_{\beta}$ must be type 2 premice.

${ }^{23}$ In this situation it would have been possible to set $B_{\alpha+1}^{*}=B_{\beta}$, and the reader might object that we are dropping information unnecessarily here. But for the cephalanxes we will produce, our proof of iterability would break down if we set $B_{\alpha+1}^{*}=B_{\beta}$, and it will turn out that we have in fact carried sufficient information (at least, for our present purposes).
} 
Proof. This is mostly like 3.21. Part 4 , when replacing ' $\mathscr{M}$ ' with ' $\mathscr{Q}$ ', etc, and when $q=-1$, follows easily from the iteration rules. For consider this situation and suppose $\alpha+1 \in \mathscr{Q}$ and $\beta=\operatorname{pred}^{\mathcal{T}}(\alpha+1) \in \mathscr{B}$ but $\alpha+1 \notin \mathscr{D}^{\mathcal{T}}$. Since $q=-1, B$ is active, so $\mathrm{OR}^{Q}=\rho$, so $\mathrm{OR}^{Q_{\beta}}=\rho_{\beta}$, so $\kappa<\rho_{\beta}$, and since $\alpha+1 \notin \mathscr{D}^{\mathcal{T}}, E_{\alpha}$ is total over $B_{\beta} \| \rho_{\beta}$, so $\kappa \leq \gamma_{\beta}$. Therefore (since $\alpha+1 \notin \mathscr{B}$ ) $\kappa=\gamma_{\beta}$ and $E_{\beta} \notin \mathbb{E}_{+}\left(M_{\beta}\right)$. Therefore $E_{\beta}=F^{Q_{\beta}}$ (as $\rho_{\beta}=\mathrm{OR}^{Q_{\beta}}$ and $Q_{\beta} \| \rho_{\beta}=$ $\left.M_{\beta} \| \rho_{\beta}\right)$. But $\nu\left(F^{Q_{\beta}}\right) \leq \gamma_{\beta}$, so $\iota_{\beta}=\gamma_{\beta}$, but by the iteration rules, $\kappa<\iota_{\beta}$, a contradiction.

3.24 Definition. Let $\mathcal{T}$ be an iteration tree on a cephal $B$ and $\alpha+1<\operatorname{lh}(\mathcal{T})$. We write $\operatorname{ex}_{\alpha}^{\mathcal{T}}$ for the active segmented-premouse $P$ such that $E_{\alpha}^{\mathcal{T}}=F^{P}$, if $B$ is a bicephalus then $P \unlhd M_{\alpha}^{\mathcal{T}}$ or $P \unlhd N_{\alpha}^{\mathcal{T}}$, and if $B$ is a cephalanx then $P \unlhd M_{\alpha}^{\mathcal{T}}$ or $P \unlhd Q_{\alpha}^{\mathcal{T}}$.

3.25 Definition. Let $B$ be a cephal. A potential tree on $B$ is a tuple

$$
\mathcal{T}=\left(<_{\mathcal{T}},\left\langle E_{\alpha}\right\rangle_{\alpha+1<\eta}\right),
$$

such that if $\eta$ is a limit then $\mathcal{T}$ is an iteration tree on $B$, and if $\eta=\gamma+1$ then $\mathcal{T} \uparrow \gamma$ is an iteration tree on $B$, and $\mathcal{T}$ satisfies all requirements of 3.20, except that we drop the requirement that $B_{\gamma}$ be a cephal or premouse, and add the requirement that $M_{\gamma}, N_{\gamma}, Q_{\gamma}, \operatorname{Ult}\left(M_{\gamma}, F^{M_{\gamma}}\right), \operatorname{Ult}\left(N_{\gamma}, F^{N_{\gamma}}\right)$, and $\operatorname{Ult}\left(Q_{\gamma}, F^{Q_{\gamma}}\right)$ are all wellfounded (if defined).

The next lemma is easy:

3.26 Lemma. Let $\mathcal{T}$ be a potential tree on a cephal $B$. Then $\mathcal{T}$ is an iteration tree. Moreover, if $\alpha<\beta<\operatorname{lh}(\mathcal{T})$ and $\beta \in \mathscr{B}^{\mathcal{T}}$ then we can apply 3.19 to $B_{\alpha}, B_{\beta}$ and the sequence of extenders used along $(\alpha, \beta]_{\mathcal{T}}$. Further, assume that if $B$ is an active cephalanx and $\operatorname{lgcd}\left(Q^{B}\right)<\nu\left(F^{Q^{B}}\right)$ then $Q^{B}$ is a premouse. Then every model of $\mathcal{T}$ is either a cephal or a premouse.

3.27 Definition (Iterability for cephals). Let $B$ be a bicephalus and $\alpha \in$ OR. The length $\theta$ iteration game for $B$ is defined in the obvious way: given $\mathcal{T}\left\lceil\alpha+1\right.$ with $\alpha+1<\theta$, player I must choose an extender $E_{\alpha}$, and given $\mathcal{T}\lceil\lambda$ for a limit $\lambda<\theta$, player II must choose $[0, \lambda]_{\mathcal{T}}$. The first player to break one of these rules or one of the conditions of 3.20 loses, and otherwise player II wins.

The iteration game for cephalanxes is defined similarly.

We say that a cephal $B$ is $\alpha$-iterable if there is a winning strategy for player II in the length $\alpha$ iteration game for $B$.

3.28 Lemma. Let $B$ be an $\left(\omega_{1}+1\right)$-iterable cephal of degree $(m, k)$. Let $\mathcal{T}$ be an iteration tree on $B$ and $\alpha<\operatorname{lh}(\mathcal{T})$. Then:

- Suppose $M_{\alpha}^{\mathcal{T}} \neq \emptyset$. If $\alpha \in \mathscr{B}^{\mathcal{T}}$ let $d=m$; otherwise let $d=\operatorname{deg}^{\mathcal{T}}(\alpha)$. Then suitable condensation holds through $\left(M_{\alpha}^{\mathcal{T}}, \max (d, 0)\right)$. 
- Suppose $B$ is a cephalanx and $Q_{\alpha}^{\mathcal{T}} \neq \emptyset$. If $\alpha \in \mathscr{B}^{\mathcal{T}}$ let $d=k$; otherwise let $d=\operatorname{deg}^{\mathcal{T}}(\alpha)$. Then suitable condensation holds below $\left(Q_{\alpha}^{\mathcal{T}}, \max (d, 0)\right)$, and if either $[0, \alpha]_{\mathcal{T}}$ drops or $Q, Q_{\alpha}^{\mathcal{T}}$ are premice, then suitable condensation holds through $\left(Q_{\alpha}^{\mathcal{T}}, \max (d, 0)\right)$.

Proof. If $\mathcal{T}$ is trivial, use 2.14 (for example, $M^{B}$ is $\left(m, \omega_{1}+1\right)$-iterable). This extends to longer trees $\mathcal{T}$ by 2.11 and the elementarity of the iteration maps.

\section{Analysis of iterable cephals}

In this section we prove the main facts about iterable bicephali and cephalanxes, which establish strong fine structural restrictions on them.

4.1 Definition. Let $m<\omega$ and $M$ a $\rho$-sound premouse with $\rho_{m+1}^{M} \leq \rho \leq \rho_{m}^{M}$. Let $\kappa<\rho$, let $H=\operatorname{cHull}_{m+1}^{M}\left(\kappa \cup \vec{p}_{m+1}^{M}\right)$ and $\pi: H \rightarrow M$ the uncollapse.

Then $M$ has an $(m, \rho)$-good core at $\kappa$ iff $H\left\|\left(\kappa^{+}\right)^{H}=M\right\|\left(\kappa^{+}\right)^{M}, H$ is $\kappa$-sound, $\operatorname{cr}(\pi)=\kappa, \pi(\kappa) \geq \rho$ and $\pi\left(p_{m+1}^{H} \backslash \kappa\right)=p_{m+1}^{M} \backslash \kappa$. In this context, let $H_{m, \kappa}^{M}=H$ and let $G_{m, \kappa, \rho}^{M}$ be the length $\rho$ extender derived from $\pi$.

4.2 Remark. Note that if $M$ has an $(m, \rho)$-good core at $\kappa$ then, with $\pi, H$ as above, we have $\rho_{m+1}^{M} \leq \kappa, M$ is not $(m+1)$-sound, $G=G_{m, \kappa, \rho}^{M}$ is weakly amenable to $H, M=\operatorname{Ult}_{m}(H, G)$ and $i_{G}^{H, m}=\pi$.

4.3 Theorem. Let $B=(\rho, M, N)$ be an $\left(\omega_{1}+1\right)$-iterable non-trivial bicephalus. Then $B$ is not sound. Let $m=m^{B}$ and $n=n^{B}$. Then exactly one of the following holds:

(a) $N$ is active type 1 or type 3 with largest cardinal $\rho$, and letting $\kappa=\operatorname{cr}\left(F^{N}\right)$, then $m \geq 0$ and $M$ has an $(m, \rho)$-good core at $\kappa$, and $G_{m, \kappa, \rho}^{M}=F^{N} \uparrow \rho$.

(b) Vice versa.

Proof. We may assume ZFC, as we can work in an inner model which contains $B$ and is closed under an iteration strategy $\Sigma$ for $B$, such as $\operatorname{HOD}_{B, \Sigma}$ or $L[B, \Sigma]$. So we may also assume $B$ is countable. We mimic the self-comparison argument used in $[3, \S 9]$. Fix an $\left(\omega_{1}+1\right)$-iteration strategy $\Sigma$ for $B$. We form a pair of padded iteration trees $(\mathcal{T}, \mathcal{U})$ on $B$, each via $\Sigma$, by comparison. We will show that the comparison terminates, using the ISC and some more. Examining the circumstances under which the comparison terminates, we will show that $B$ is unsound, and the comparison produces an iterate $B^{\prime}$ of $B$, also a cephal, such that $B^{\prime}$ has a good core. A new feature of the proof (in contrast to the classical phalanx comparisons) is that we then need to show that the iteration map from $B$ to $B^{\prime}$ cannot introduce this property, $\operatorname{so} B$ also has a good core.

The trees $(\mathcal{T}, \mathcal{U})$ may be padded, but for each $\alpha$ we will have either $E_{\alpha}^{\mathcal{T}} \neq \emptyset$ or $E_{\alpha}^{\mathcal{U}} \neq \emptyset$. See $\S 1.1 .6$ regarding padding and tree ordering. At stage $\alpha$ of the comparison, given $\alpha \in \mathscr{B}^{\mathcal{T}}$, we may set $E_{\alpha}^{\mathcal{T}}=\emptyset$, and simultaneously declare that, if $\mathcal{T}$ is to later use a non-empty extender, then letting $\beta>\alpha$ be least such 
that $E_{\beta}^{\mathcal{T}} \neq \emptyset$, we will have $E_{\beta}^{\mathcal{T}} \in \mathbb{E}_{+}\left(M_{\alpha}^{\mathcal{T}}\right)=\mathbb{E}_{+}\left(M_{\beta}^{\mathcal{T}}\right)$. Or instead, we may declare that $E_{\beta}^{\mathcal{T}} \in \mathbb{E}_{+}\left(N_{\alpha}^{\mathcal{T}}\right)$. Toward this, we define non-empty sets

$$
\mathfrak{M}_{\beta}^{\mathcal{T}} \subseteq\left\{M_{\beta}^{\mathcal{T}}, N_{\beta}^{\mathcal{T}}\right\} \backslash\{\emptyset\} .
$$

We will require that if $E_{\beta}^{\mathcal{T}} \neq \emptyset$, then $E_{\beta}^{\mathcal{T}} \in \mathbb{E}_{+}(P)$ for some $P \in \mathfrak{M}_{\beta}^{\mathcal{T}}$. All models in $\mathfrak{M}_{\beta}^{\mathcal{T}}$ will be non-empty.

We also define sets $S_{\beta}^{\mathcal{T}} \subseteq t_{\beta}^{\mathcal{T}} \subseteq\{0,1\}$ for convenience. Let $0 \in t_{\beta}^{\mathcal{T}}$ iff $M_{\beta}^{\mathcal{T}} \neq \emptyset$, and $1 \in t_{\beta}^{\mathcal{T}}$ iff $N_{\beta}^{\mathcal{T}} \neq \emptyset$. Let $0 \in S_{\beta}^{\mathcal{T}}$ iff $M_{\beta}^{\mathcal{T}} \in \mathfrak{M}_{\beta}^{\mathcal{T}}$, and $1 \in S_{\beta}^{\mathcal{T}}$ iff $N_{\beta}^{\mathcal{T}} \in \mathfrak{M}_{\beta}^{\mathcal{T}}$. (We will explicitly define either $\mathfrak{M}_{\beta}^{\mathcal{T}}$ or $S_{\beta}^{\mathcal{T}}$, implicitly defining the other.)

The preceding definitions also extend to $\mathcal{U}$.

We start with $B_{0}^{\mathcal{T}}=B=B_{0}^{\mathcal{U}}$ and $S_{0}^{\mathcal{T}}=\{0,1\}=S_{0}^{\mathcal{U}}$.

Suppose we have defined $(\mathcal{T}, \mathcal{U})\lceil\lambda$ for some limit $\lambda$. Then $(\mathcal{T}, \mathcal{U})\lceil\lambda+1$ is determined by $\Sigma$, and $S_{\lambda}^{\mathcal{T}}=\lim _{\alpha<\mathcal{}} S_{\alpha}^{\mathcal{T}}$, and $S_{\lambda}^{\mathcal{U}}$ is likewise.

Now suppose we have defined $(\mathcal{T}, \mathcal{U})\left\lceil\alpha+1\right.$ and $S_{\alpha}^{\mathcal{T}}$ and $S_{\alpha}^{\mathcal{U}}$; we determine what to do next (at stage $\alpha$ ).

Let $\widetilde{\nu}(F)=\nu(F)$ for $F$ an extender, and $\widetilde{\nu}(\emptyset)=\infty$ (with $\infty>\alpha$ for $\alpha \in \mathrm{OR}$ ). CASE 1. There is $\xi \in \mathrm{OR}$ such that for some $Y \in \mathfrak{M}_{\alpha}^{\mathcal{T}}$ and $Z \in \mathfrak{M}_{\alpha}^{\mathcal{U}}$, we have $\xi \leq \mathrm{OR}^{Y} \cap \mathrm{OR}^{Z}$ and $Y|\xi \neq Z| \xi$.

Let $\xi$ be least such and $\nu=$ the minimum value of $\min \left(\widetilde{\nu}\left(F^{Y \mid \xi}\right), \widetilde{\nu}\left(F^{Z \mid \xi}\right)\right)$ over all choices of pairs $(Y, Z)$ witnessing the choice of $\xi$ (there are at most 4 ). SubCASE 1.1. For some $(Y, Z)$ witnessing the choice of $\xi, Y \mid \xi$ and $Z \mid \xi$ are both active and $\nu\left(F^{Y \mid \xi}\right)=\nu\left(F^{Z \mid \xi}\right)=\nu$.

Fix such $Y, Z$. We set $E_{\alpha}^{\mathcal{T}}=F^{Y \mid \xi}$ and $E_{\alpha}^{\mathcal{U}}=F^{Z \mid \xi}$. This determines $(\mathcal{T}, \mathcal{U}) \uparrow$ $\alpha+2$. Also set $S_{\alpha+1}^{\mathcal{T}}=t_{\alpha+1}^{\mathcal{T}}$ and $S_{\alpha+1}^{\mathcal{U}}=t_{\alpha+1}^{\mathcal{U}}$.

SubCASE 1.2. Otherwise.

Then take $Y, Z$ witnessing the choice of $\xi$ and such that either (i) $Y \mid \xi$ is active, $\nu\left(F^{Y \mid \xi}\right)=\nu$, and if $Z \mid \xi$ is active then $\nu\left(F^{Z \mid \xi}\right)>\nu$; or (ii) vice versa.

Say $Y \mid \xi$ is active with $\nu\left(F^{Y \mid \xi}\right)=\nu$. Then we set $E_{\alpha}^{\mathcal{T}}=F^{Y \mid \xi}$ and $E_{\alpha}^{\mathcal{U}}=\emptyset$. This determines $(\mathcal{T}, \mathcal{U})\left\lceil\alpha+2\right.$. Set $S_{\alpha+1}^{\mathcal{T}}=t_{\alpha+1}^{\mathcal{T}}$. Now suppose there is $X \in \mathfrak{M}_{\alpha}^{\mathcal{U}}$ with $X \mid \xi$ active and $\nu\left(F^{X \mid \xi}\right)=\nu$. Then $X|\xi=Y| \xi$, so we must avoid setting $E_{\beta}^{\mathcal{U}}=F^{X \mid \xi}$ at some $\beta>\alpha$. So we set $\mathfrak{M}_{\alpha+1}^{\mathcal{U}}=\{Z\}$, and set $S_{\alpha+1}^{\mathcal{U}}$ accordingly. If there is no such $X$ then set $S_{\alpha+1}^{\mathcal{U}}=S_{\alpha}^{\mathcal{U}}$. (In any case, later extenders used in $\mathcal{U}$ will be incompatible with $E_{\alpha}^{\mathcal{T}}$.) The remaining cases are covered by symmetry.

CASE 2. Otherwise.

Then we stop the comparison at stage $\alpha$.

This completes the definition of $(\mathcal{T}, \mathcal{U})$. For $\alpha<\ln (\mathcal{T}, \mathcal{U})$, let $S^{\mathcal{T}}(\alpha)$ be the largest $\beta \leq_{\mathcal{T}} \alpha$ such that $S_{\beta}^{\mathcal{T}}=\{0,1\}$; here if $\alpha \in \mathscr{B}^{\mathcal{T}}$ then $B_{\beta}^{\mathcal{T}}=B_{\alpha}^{\mathcal{T}}$. Let $S^{\mathcal{U}}(\alpha)$ be likewise. For $\alpha+1<\operatorname{lh}(\mathcal{T}, \mathcal{U})$, let $\operatorname{lh}_{\alpha}=\operatorname{lh}\left(E_{\alpha}^{\mathcal{T}}\right)$ and $\nu_{\alpha}=\nu\left(E_{\alpha}^{\mathcal{T}}\right)$ if $E_{\alpha}^{\mathcal{T}} \neq \emptyset$, and $\operatorname{lh}_{\alpha}=\operatorname{lh}\left(E_{\alpha}^{\mathcal{U}}\right)$ and $\nu_{\alpha}=\nu\left(E_{\alpha}^{\mathcal{U}}\right)$ otherwise. (Note that if $E_{\alpha}^{\mathcal{T}} \neq \emptyset \neq$ $E_{\alpha}^{\mathcal{U}}$ then $\operatorname{lh}\left(E_{\alpha}^{\mathcal{T}}\right)=\operatorname{lh}\left(E_{\alpha}^{\mathcal{U}}\right)$ and $\left.\nu\left(E_{\alpha}^{\mathcal{T}}\right)=\nu\left(E_{\alpha}^{\mathcal{U}}\right).\right)$

Claim 1. Let $\alpha+1, \beta+1<\operatorname{lh}(\mathcal{T}, \mathcal{U})$. Then (i) if $\alpha<\beta$ then $\operatorname{lh}_{\alpha} \leq \operatorname{lh}_{\beta}$ and $\nu_{\alpha}<\nu_{\beta}$; and (ii) if $E_{\alpha}^{\mathcal{T}} \neq \emptyset \neq E_{\beta}^{\mathcal{U}}$ then $E_{\alpha}^{\mathcal{T}}\left\lceil\nu_{\alpha} \neq E_{\beta}^{\mathcal{U}}\left\lceil\nu_{\beta}\right.\right.$. 
Proof. Part (i) is by standard considerations together with the definition of $\mathfrak{M}_{\alpha+1}^{\mathcal{U}}$ in Subcase 1.2 above (which prevents having $E_{\alpha+1}^{\mathcal{U}}=E_{\alpha}^{\mathcal{T}}$, for example). Part (ii): If $\alpha+1=\beta+1$, this is directly by construction, and if $\alpha+1<\beta+1$, use part (i) and the ISC as usual.

Claim 2. The comparison terminates at some countable stage.

Proof. By the proof that standard comparison terminates, with Claim 1.

So let $\alpha$ be such that the comparison stops at stage $\alpha$.

Claim 3. $\operatorname{card}\left(S_{\alpha}^{\mathcal{T}}\right)=\operatorname{card}\left(S_{\alpha}^{\mathcal{U}}\right)=1$ and $\mathfrak{M}_{\alpha}^{\mathcal{T}}=\mathfrak{M}_{\alpha}^{\mathcal{U}}$.

Proof. If $\alpha \in \mathscr{B}^{\mathcal{T}}$ then $B_{\alpha}^{\mathcal{T}}$ is non-trivial, by 3.26; likewise for $\mathcal{U}$. So because Case 2 attains at stage $\alpha$, we do not have $S_{\alpha}^{\mathcal{T}}=S_{\alpha}^{\mathcal{U}}=\{0,1\}$.

It is not true that $(\dagger) P \triangleleft Q$ or $Q \triangleleft P$ for some $P \in \mathfrak{M}_{\alpha}^{\mathcal{T}}$ and $Q \in \mathfrak{M}_{\alpha}^{\mathcal{U}}$. For suppose $(\dagger)$ holds; we may assume $Q \triangleleft P$. Then $Q$ is sound, so by $3.21, \alpha \in \mathscr{B}^{\mathcal{U}}$, so by $(\dagger)$ and Case 2 hypothesis, $\operatorname{card}\left(S_{\alpha}^{\mathcal{U}}\right)=1$. Say $S_{\alpha}^{\mathcal{U}}=\{0\}$. Let $\beta=S^{\mathcal{U}}(\alpha)$. Then $B_{\beta}^{\mathcal{U}}=B_{\alpha}^{\mathcal{U}}$ and $E_{\beta}^{\mathcal{T}} \in \mathbb{E}_{+}\left(N_{\beta}^{\mathcal{U}}\right)$ and $E_{\gamma}^{\mathcal{U}}=\emptyset$ for all $\gamma \in[\beta, \alpha)$. Let $\varrho=\rho_{\beta}^{\mathcal{U}}$. Then $\operatorname{lh}_{\beta}^{\mathcal{T}} \geq\left(\varrho^{+}\right)^{B_{\beta}^{\mathcal{U}}}$. So $\mathcal{P}(\varrho) \cap P=\mathcal{P}(\varrho) \cap B_{\beta}^{\mathcal{U}}$, contradicting the fact that $M_{\beta}^{\mathcal{U}}=Q \triangleleft P$.

Now suppose that $S_{\alpha}^{\mathcal{T}}=\{0,1\}$ but $\operatorname{card}\left(S_{\alpha}^{\mathcal{U}}\right)=1$. Let $\delta$ be least such that $M_{\alpha}^{\mathcal{T}}\left|\delta \neq N_{\alpha}^{\mathcal{T}}\right| \delta$. Let $Q \in \mathfrak{M}_{\alpha}^{\mathcal{U}}$. Then $Q \triangleleft M_{\alpha}^{\mathcal{T}}\left\|\delta=N_{\alpha}^{\mathcal{T}}\right\| \delta$, so (†) holds, contradiction. So $\operatorname{card}\left(S_{\alpha}^{\mathcal{T}}\right)=\operatorname{card}\left(S_{\alpha}^{\mathcal{U}}\right)=1$, and because $(\dagger)$ fails, $\mathfrak{M}_{\alpha}^{\mathcal{T}}=$ $\mathfrak{M}_{\alpha}^{\mathcal{U}}$.

Claim 4. $\alpha \in \mathscr{B}^{\mathcal{T}} \Delta \mathscr{B}^{\mathcal{U}}$.

Proof. Either $\mathcal{T}$ or $\mathcal{U}$ is non-padded cofinally in $\alpha$ (that is, if $\alpha=\beta+1$ then either $E_{\beta}^{\mathcal{T}} \neq \emptyset$ or $E_{\beta}^{\mathcal{U}} \neq \emptyset$, and if $\alpha$ is a limit then either $E_{\beta}^{\mathcal{T}} \neq \emptyset$ for cofinally many $\beta<\alpha$, or $E_{\beta}^{\mathcal{U}} \neq \emptyset$ for cofinally many $\beta<\alpha$ ). By this and Claim 3, we get $\alpha \notin \mathscr{B}^{\mathcal{T}} \cap \mathscr{B}^{\mathcal{U}}$, so assume that $\alpha \notin \mathscr{B}^{\mathcal{T}} \cup \mathscr{B}^{\mathcal{U}}$. Then standard calculations using 3.21 give that $\mathcal{T}, \mathcal{U}$ use compatible extenders, a contradiction.

By the previous claims, we can assume $\alpha \in \mathscr{B}^{\mathcal{T}} \backslash \mathscr{B}^{\mathcal{U}}, S_{\alpha}^{\mathcal{T}}=\{0\}$ and $S_{\alpha}^{\mathcal{U}}=$ $\{1\}$, so $\widetilde{B}=B_{\alpha}^{\mathcal{T}}$ is a bicephalus, $\alpha \in \mathscr{N}^{\mathcal{U}}$, and $M_{\alpha}^{\mathcal{T}}=N_{\alpha}^{\mathcal{U}}$; the other cases are almost symmetric. We will show conclusion (a) of the theorem holds; under symmetric assumptions (b) can hold instead. Let $\beta=S^{\mathcal{T}}(\alpha)$. Let $\widetilde{\rho}=\rho(\widetilde{B})$. Then $\widetilde{B}=B_{\beta}^{\mathcal{T}}$ and for all $\gamma \in[\beta, \alpha)$, we have $E_{\gamma}^{\mathcal{T}}=\emptyset \neq E_{\gamma}^{\mathcal{U}}$ and $\left(\widetilde{\rho}^{+}\right)^{\widetilde{B}} \leq \operatorname{lh}_{\gamma}^{\mathcal{U}}$. Claim 5. $\alpha=\beta+1$ and $\operatorname{lh}_{\beta}^{\mathcal{U}}=\left(\widetilde{\rho}^{+}\right)^{\widetilde{B}}$ and $E_{\beta}^{\mathcal{U}}$ is type 1 or type 3 .

Proof. Suppose not. Then by $3.21, N_{\alpha}^{\mathcal{U}}$ is not $\widetilde{\rho}$-sound (recall that if $\alpha>\beta+1$ and $\operatorname{lh}_{\beta+1}^{\mathcal{U}}=\operatorname{lh}_{\beta}^{\mathcal{U}}$ then $E_{\beta+1}^{\mathcal{U}}$ is type 2). But by $3.21, M_{\alpha}^{\mathcal{T}}$ is $\widetilde{\rho}$-sound. So $M_{\alpha}^{\mathcal{T}} \neq N_{\alpha}^{\mathcal{U}}$, contradiction. 
Let $\widetilde{B}=(\widetilde{\rho}, \widetilde{M}, \widetilde{N})=B_{\alpha}^{\mathcal{T}}=B_{\beta}^{\mathcal{T}}$. Since $E_{\beta}^{\mathcal{U}} \in \mathbb{E}_{+}(\widetilde{N})$, and $\operatorname{lh}_{\beta}^{\mathcal{U}}=\left(\widetilde{\rho}^{+}\right)^{\widetilde{B}}$, $\widetilde{N} \mid\left(\widetilde{\rho}^{+}\right)^{\widetilde{B}}$ projects to $\widetilde{\rho}$, so $\mathrm{OR}^{\widetilde{N}}=\left(\widetilde{\rho}^{+}\right)^{\widetilde{B}}$ and $F^{\widetilde{N}}=E_{\beta}^{\mathcal{U}}$. Let $\widetilde{F}=F^{\widetilde{N}}$ and $\widetilde{\kappa}=\operatorname{cr}(\widetilde{F})$. It follows that (a) of the theorem holds regarding $\widetilde{B}$; using the iteration embeddings we will deduce that $B$ is not sound, and (a) holds regarding $B$. Note that either $\operatorname{OR}(\widetilde{M})>\operatorname{OR}(\widetilde{N})$, or $\operatorname{OR}(\widetilde{M})=\operatorname{OR}(\widetilde{N}), \widetilde{N}$ has superstrong type and $\widetilde{M}$ is type 2 ; in either case $m \geq 0$. Also $\mathrm{OR}^{N}=\left(\rho^{+}\right)^{B}$ and $N$ is active with $F=F^{N}$, a preimage of $\widetilde{F}$. Let $\kappa=\operatorname{cr}(F)$; so $\kappa<\rho$.

Claim 6. $M$ is not $m+1$-sound, so $B$ is not sound.

Proof. Suppose $M$ is $m+1$-sound. Let $z=z_{m+1}^{M}$ and $\zeta=\zeta_{m+1}^{M}$. By [12, 2.17], $z=p_{m+1}^{M}$ and $\zeta=\rho_{m+1}^{M} \leq \rho$. So $\kappa \in \operatorname{Hull}_{m+1}^{M}\left(\zeta \cup z \cup \vec{p}_{m}^{M}\right)$. Let $\widetilde{z}=z_{m+1}^{\widetilde{M}}$ and $\widetilde{\zeta}=\zeta_{m+1}^{\widetilde{M}}$. By $[12,2.20], \widetilde{z}=i_{0 \alpha}^{\mathcal{T}}(z)$ and $\widetilde{\zeta}=\sup i_{0 \alpha}^{\mathcal{T}}$ " $\zeta$, so $\widetilde{\zeta} \leq \widetilde{\rho}$ and

$$
i_{0 \alpha}^{\mathcal{T}}(\kappa) \in \operatorname{Hull}_{m+1}^{\widetilde{M}}\left(\widetilde{\zeta} \cup \widetilde{z} \cup \vec{p}_{m}^{\widetilde{M}}\right) .
$$

Let $\widetilde{H}=N_{\alpha}^{* \mathcal{U}}$. Then $\widetilde{M}=N_{\alpha}^{\mathcal{U}}=\operatorname{Ult}_{m}(\widetilde{H}, \widetilde{F})$ and $\widetilde{\zeta}=\sup i \widetilde{H}$ " $\zeta^{\widetilde{H}}$, and since $\widetilde{\zeta} \leq \widetilde{\rho}$, therefore $\widetilde{\zeta} \leq \widetilde{\kappa}$. Also, $\widetilde{z}=i \widetilde{F}\left(z_{m+1}^{\widetilde{H}}\right)$. But $\widetilde{\kappa} \notin \operatorname{rg}\left(i \frac{\widetilde{H}}{\widetilde{F}}\right)$, so

$$
\widetilde{\kappa} \notin \operatorname{Hull}_{m+1}^{\widetilde{M}}\left(\widetilde{\zeta} \cup \widetilde{z} \cup \vec{p}_{m}^{\widetilde{M}}\right) .
$$

But $i_{0 \alpha}^{\mathcal{T}}\left\lceil\rho=j_{0 \alpha}^{\mathcal{T}}\left\lceil\rho\right.\right.$, so $i_{0 \alpha}^{\mathcal{T}}(\kappa)=\widetilde{\kappa}$, contradicting lines (1) and (2).

We can now complete the proof:

Claim 7. Conclusion (a) of the theorem holds.

Proof. Suppose $N$ is type 1. Let $\widetilde{p}=p_{m+1}^{\widetilde{M}} \backslash \widetilde{\rho}$ and $\widetilde{H}=\operatorname{cHull}_{m+1}^{\widetilde{M}}\left(\widetilde{\kappa} \cup \widetilde{p} \cup \vec{p}_{m}^{\widetilde{M}}\right)$ and $\tilde{\pi}: \widetilde{H} \rightarrow \widetilde{M}$ the uncollapse. Then $\widetilde{H}=N_{\alpha}^{* \mathcal{U}}, \tilde{\pi}=j_{\alpha}^{* \mathcal{U}}, \widetilde{H}$ is $\widetilde{\kappa}$-sound and letting $\widetilde{q}=p_{m+1}^{\widetilde{H}} \backslash \widetilde{\kappa}$, we have $\tilde{\pi}(\widetilde{q})=\widetilde{p}$ and $\rho_{m}^{\widetilde{M}}=\sup \tilde{\pi} " \rho_{m}^{\widetilde{H}}$ and

$$
\widetilde{H}\left\|\left(\widetilde{\kappa}^{+}\right)^{\widetilde{H}}=\widetilde{M}\right\|\left(\widetilde{\kappa}^{+}\right)^{\widetilde{M}}=\widetilde{N} \|\left(\widetilde{\kappa}^{+}\right)^{\widetilde{N}} .
$$

We have $\kappa, H, \pi$ as in (a); let $p=p_{m+1}^{M} \backslash \rho$. We show $\left(\sup \pi " \rho_{m}^{H}\right)=\rho_{m}^{M}$. Let $\gamma<\rho_{m}^{M}$. We have $\left(\sup \widetilde{\pi} " \rho_{m}^{\widetilde{H}}\right)=\rho_{m}^{\widetilde{M}}$. So letting $i=i_{0 \alpha}^{\mathcal{T}}$,

$$
\widetilde{M} \models \text { "There is } \beta>i(\gamma) \text { with } \beta \in \operatorname{Hull}_{m+1}\left(\widetilde{\kappa} \cup\left\{\widetilde{p}, \vec{p}_{m}^{\widetilde{M}}\right\}\right) \text { ", }
$$

an $\mathrm{r} \Sigma_{m+1}$ assertion about $i\left(\gamma, \kappa, p, p_{m}^{M}\right)$, which pulls back to $M$, which suffices.

So $\pi: H \rightarrow M$ is an $m$-embedding. Let $\pi\left(p^{H}\right)=p$. Let $\left\langle H_{\gamma}\right\rangle_{\gamma<\rho_{m}^{H}}$ be the natural stratification of $\operatorname{Hull}_{m+1}^{H}\left(\kappa \cup\left\{p^{H}, \vec{p}_{m}^{H}\right\}\right)$ (the uncollapsed hull), and $M_{\pi(\gamma)}=\pi$ " $H_{\gamma}$ and $\pi_{\gamma}: H_{\gamma} \rightarrow M_{\pi(\gamma)}$ be the restriction of $\pi$. (For example if $m=0$ and $M$ is passive,

$$
H_{\gamma}=\operatorname{Hull}_{1}^{H \mid \gamma}\left(\kappa \cup\left\{p^{H}\right\}\right) .
$$


If $M$ is active or $m>0$ use the stratification of $\mathrm{r} \Sigma_{m+1}$ truth described in [3, $\left.\S 2\right]$. Note $H_{\gamma}$ need not be transitive.) So $H=\bigcup_{\gamma<\rho_{\gamma}^{H}} H_{\gamma}$. For $\gamma$ large enough we have $\kappa \in H_{\gamma}$ and $H_{\gamma}$ is transitive below $\left(\kappa^{+}\right)^{H_{\gamma}}$, so $\pi_{\gamma}\lceil\mathcal{P}(\kappa) \subseteq \pi$, and in particular $\kappa^{\prime}=\pi(\kappa)=\pi_{\gamma}(\kappa)$. For such $\gamma$, let $E_{\gamma}$ be the (short) $\left(\kappa, \kappa^{\prime}\right)$-extender derived from $\pi_{\gamma}$. Then $H_{\gamma}, E_{\gamma} \in M\left(\operatorname{as} \sup \left(\pi_{\gamma} " \rho_{m}^{H}\right)<\rho_{m}^{M}\right)$ and $\left(\kappa^{+}\right)^{H_{\gamma}}<\left(\kappa^{+}\right)^{M}$. Let $\tilde{\pi}_{\gamma}: \widetilde{H}_{\gamma} \rightarrow \widetilde{M}_{\gamma}$ and $\widetilde{\kappa}^{\prime}$ and $\widetilde{E}_{\gamma}$ be defined likewise over $\widetilde{M}$ (for large enough $\left.\gamma<\rho_{m}^{\widetilde{H}}\right)$. We have $\left(\widetilde{H}_{\gamma} \sim \widetilde{M}\right) \|\left(\widetilde{\kappa}^{+}\right)^{\widetilde{H}_{\gamma}}$ and $\widetilde{E}_{\gamma} \uparrow \widetilde{\rho} \subseteq F^{\widetilde{N}}$ for each $\gamma$; the former is because by $2.13, \widetilde{N} \models$ "Lemma 2.13 holds for my proper segments".

Now $i " \operatorname{rg}(\pi) \subseteq \operatorname{rg}(\widetilde{\pi})$ since $i=i_{0 \alpha}^{\mathcal{T}}$ is an $m$-embedding and $i(\kappa, p)=(\widetilde{\kappa}, \widetilde{p})$. So for all $\gamma<\rho_{m}^{H}$, we have $i(\pi(\gamma)) \in \operatorname{rg}(\widetilde{\pi})$. And note that $i\left(\kappa^{\prime}\right)=\widetilde{\kappa}^{\prime}$ and if $\gamma<\rho_{m}^{H}$ is sufficiently large and $\widetilde{\pi}(\widetilde{\gamma})=i(\pi(\gamma))$, then $i\left(H_{\gamma}\right)=\widetilde{H}_{\widetilde{\gamma}}$ and $i\left(E_{\gamma}\right)=$ $\widetilde{E}_{\widetilde{\gamma}}$. Also $\rho_{m}^{\widetilde{M}}=\sup i " \rho_{m}^{M}$ and $\mathrm{OR}^{\widetilde{N}}=\sup j " \mathrm{OR}^{N}$ and $i, j$ are continuous at $\left(\kappa^{+}\right)^{N}=\left(\kappa^{+}\right)^{M}$ and $j^{\prime \prime} F^{N} \subseteq F^{\widetilde{N}}$. It follows easily that $\left(H_{\gamma} \sim M\right) \|\left(\kappa^{+}\right)^{H_{\gamma}}$ and $E_{\gamma}\left\lceil\rho \subseteq F^{N}\right.$ for all sufficiently large $\gamma<\rho_{m}^{H}$. Therefore $H\left\|\left(\kappa^{+}\right)^{H}=M\right\|\left(\kappa^{+}\right)^{M}$ and $F^{N}\lceil\rho$ is derived from $\pi$.

So $F^{N}$ is weakly amenable to $H, M=\operatorname{Ult}_{m}\left(H, F^{N}\right)$, and $\pi=i_{F^{N}}^{M, m}$ (we can factor $\pi: H \rightarrow M$ through $\operatorname{Ult}_{m}\left(H, F^{N}\right)$, and $\nu\left(F^{N}\right)=\rho$ ). So by [12], $\pi\left(z_{m+1}^{H}\right)=z_{m+1}^{M}$, but $z_{m+1}^{M} \backslash \rho=p_{m+1}^{M} \backslash \rho$, and therefore $z_{m+1}^{H} \backslash \kappa=p_{m+1}^{H} \backslash \kappa$, so $H$ is $\kappa$-sound. This completes the proof assuming that $N$ is type 1 .

If instead, $N$ is type 3 , then almost the same argument works.

This completes the proof of the theorem.

We now move on to analogues of 4.3 for cephalanxes.

4.4 Definition. Let $B$ be a passive cephalanx of degree $(m, q)$ and let $N=N^{B}$. We say that $B$ has a good core iff $m \geq 0$ and $N$ is active and letting $F=F^{N}$, $\kappa=\operatorname{cr}(F)$ and $\nu=\nu(F)$, we have: (i) $\mathrm{OR}^{N}=\rho^{+M}$, (ii) $N$ is type 1 or 3 , (iii) $M$ has an $(m, \nu)$-good core at $\kappa$, (iv) $G_{m, \kappa, \nu}^{M}=F\lceil\nu$, and (v) if $N$ is type 1 then $H_{m, \kappa}^{M}=Q$ and $m=q$.

4.5 Theorem. Let $B=(\gamma, \rho, M, Q)$ be an $\left(\omega_{1}+1\right)$-iterable, non-trivial, passive cephalanx of degree $(m, q)$. Then $B$ has a good core, so $B$ is not sound (that is, $M$ is not $(m+1)$-sound $)$.

Proof. The proof is based on that of 4.3. The main difference occurs in the rules guiding the comparison. We may assume $B$ is countable. We define padded trees $\mathcal{T}, \mathcal{U}$ on $B$, and sets $S_{\alpha}^{\mathcal{T}}, S_{\alpha}^{\mathcal{U}}, \mathfrak{M}_{\alpha}^{\mathcal{T}}, \mathfrak{M}_{\alpha}^{\mathcal{U}}$, much as before. We start with $S_{0}^{\mathcal{T}}=S_{0}^{\mathcal{U}}=\{0,1\}$. At limit stages, proceed as in 4.3. Suppose we have defined $(\mathcal{T}, \mathcal{U})\left\lceil\alpha+1, S_{\alpha}^{\mathcal{T}}\right.$ and $S_{\alpha}^{\mathcal{U}}$ and if $\operatorname{card}\left(S_{\alpha}^{\mathcal{T}}\right)=\operatorname{card}\left(S_{\alpha}^{\mathcal{U}}\right)=1$ then $B_{\alpha}^{\mathcal{T}} \nsupseteq B_{\alpha}^{\mathcal{U}} \nsupseteq B_{\alpha}^{\mathcal{T}}$ (otherwise the comparison has already terminated).

CASE 1. $\operatorname{card}\left(S_{\alpha}^{\mathcal{T}}\right)=\operatorname{card}\left(S_{\alpha}^{\mathcal{U}}\right)=1$.

Choose extenders as usual (as in 4.3).

CASE 2. $S_{\alpha}^{\mathcal{T}}=\{0,1\}$ and if $S_{\alpha}^{\mathcal{U}}=\{0,1\}$ then $\rho_{\alpha}^{\mathcal{T}} \leq \rho_{\alpha}^{\mathcal{U}}$. 
So $B_{\alpha}^{\mathcal{T}}$ is a cephalanx; let $B^{\mathcal{T}}=\left(\gamma^{\mathcal{T}}, \rho^{\mathcal{T}}, M^{\mathcal{T}}, Q^{\mathcal{T}}\right)=B_{\alpha}^{\mathcal{T}}$. Let $B^{\mathcal{U}}=B_{\alpha}^{\mathcal{U}}$. We will have by induction that for every $\beta<\alpha, \operatorname{lh}_{\beta}^{\mathcal{T}} \leq \rho^{\mathcal{T}}$ and $\operatorname{lh}_{\beta}^{\mathcal{U}} \leq \rho^{\mathcal{T}}$. Since $B$ is passive, $B^{\mathcal{T}} \mid \rho^{\mathcal{T}}$ and $B^{\mathcal{U}} \mid \rho^{\mathcal{T}}$ are well-defined premice.

SubCASE 2.1. $B^{\mathcal{T}}\left|\rho^{\mathcal{T}} \neq B^{\mathcal{U}}\right| \rho^{\mathcal{T}}$.

Choose extenders as usual.

Suppose $B^{\mathcal{T}}\left|\rho^{\mathcal{T}}=B^{\mathcal{U}}\right| \rho^{\mathcal{T}}$. We say in $\mathcal{T}$ we move into $M^{\mathcal{T}}$ if either $\left[E_{\alpha}^{\mathcal{T}} \neq \emptyset\right.$ and $\left.E_{\alpha}^{\mathcal{T}} \in \mathbb{E}_{+}\left(M^{\mathcal{T}}\right)\right]$ or $\left[E_{\alpha}^{\mathcal{T}}=\emptyset\right.$ and $\left.S_{\alpha+1}^{\mathcal{T}}=\{0\}\right]$. Likewise move into $Q^{\mathcal{T}}$, and likewise with regard to $\mathcal{U}$ if $S_{\alpha}^{\mathcal{U}}=\{0,1\}$. In each case below we will move into some model in $\mathcal{T}$; we may do likewise for $\mathcal{U}$. These choices produce premice $R, S$ from which to choose $E_{\alpha}^{\mathcal{T}}, E_{\alpha}^{\mathcal{U}}$, as in the proof of $4.3,{ }^{24}$, given that $R \notin S \nsupseteq R$ (for example, if $S_{\alpha}^{\mathcal{U}}=\{1\}$ and in $\mathcal{T}$ we move into $M^{\mathcal{T}}$, then $R=M^{\mathcal{T}}$ and $S=Q^{\mathcal{U}}$ ). If $R \unlhd S$ or $S \unlhd R$ then we terminate the comparison, saying the comparison terminates early. If $B^{\mathcal{U}}$ is a cephalanx and we do not move into any model in $\mathcal{U}$ and $E_{\alpha}^{\mathcal{U}}=\emptyset$ then we set $S_{\alpha+1}^{\mathcal{U}}=\{0,1\}$. SubCASE 2.2. $\operatorname{card}\left(S_{\alpha}^{\mathcal{U}}\right)=1$ and $B^{\mathcal{T}}\left|\rho^{\mathcal{T}}=B^{\mathcal{U}}\right| \rho^{\mathcal{T}}$.

Let $\{P\}=\mathfrak{M}_{\alpha}^{\mathcal{U}}$. If $Q^{\mathcal{T}} \unlhd P$ move into $M^{\mathcal{T}}$ in $\mathcal{T}$; if $Q^{\mathcal{T}} \unlhd P$ move into $Q^{\mathcal{T}}$. Subcase 2.3. $S_{\alpha}^{\mathcal{T}}=S_{\alpha}^{\mathcal{U}}=\{0,1\}$ and $B^{\mathcal{T}}\left|\rho^{\mathcal{T}}=B^{\mathcal{U}}\right| \rho^{\mathcal{T}}$.

Let $\left(\gamma^{\mathcal{U}}, \rho^{\mathcal{U}}, M^{\mathcal{U}}, Q^{\mathcal{U}}\right)=B^{\mathcal{U}}$. So $\rho^{\mathcal{T}} \leq \rho^{\mathcal{U}}$. Then:

- If $Q^{\mathcal{T}}=Q^{\mathcal{U}}$ and $\rho^{\mathcal{T}}=\rho^{\mathcal{U}}$ : Move into $Q^{\mathcal{T}}$ in $\mathcal{T}$ and $M^{\mathcal{U}}$ in $\mathcal{U}$.

- If $Q^{\mathcal{T}}=Q^{\mathcal{U}}$ and $\rho^{\mathcal{T}}<\rho^{\mathcal{U}}$ : Move into $M^{\mathcal{T}}$ in $\mathcal{T}$, and if also $M^{\mathcal{T}} \mid \rho^{\mathcal{U}}=$ $B^{\mathcal{U}} \mid \rho^{\mathcal{U}}$ then move into $Q^{\mathcal{U}}$ in $\mathcal{U}$.

- If $Q^{\mathcal{T}} \triangleleft Q^{\mathcal{U}}$ : Move into $M^{\mathcal{T}}$ in $\mathcal{T}$ (note here $\rho^{\mathcal{T}}<\rho^{\mathcal{U}}$ and $Q^{\mathcal{T}} \triangleleft B^{\mathcal{U}} \| \rho^{\mathcal{U}}$ ).

- If $Q^{\mathcal{U}} \triangleleft Q^{\mathcal{T}}$ : Move into $Q^{\mathcal{T}}$ in $\mathcal{T}$ and $M^{\mathcal{U}}$ in $\mathcal{U}$ (note here $\rho^{\mathcal{T}} \leq \gamma^{\mathcal{U}}<\rho^{\mathcal{U}}$ ).

- If $Q^{\mathcal{T}} \rrbracket Q^{\mathcal{U}} \rrbracket Q^{\mathcal{T}}$ : Move into $Q^{\mathcal{T}}$ in $\mathcal{T}$; if also $Q^{\mathcal{T}}\left|\rho^{\mathcal{U}}=B^{\mathcal{U}}\right| \rho^{\mathcal{U}}$, move into $Q^{\mathcal{U}}$ in $\mathcal{U}$.

The remaining cases are by symmetry. Define $\mathrm{lh}_{\alpha}$ and $\nu_{\alpha}$ as for 4.3.

Claim 1. Let $\alpha<\beta<\operatorname{lh}(\mathcal{T}, \mathcal{U})$. Then (i) if $\beta+1<\operatorname{lh}(\mathcal{T}, \mathcal{U})$ then $\operatorname{lh}_{\alpha} \leq \operatorname{lh}_{\beta}$ and $\nu_{\alpha}<\nu_{\beta}$; and (ii) if $S_{\beta}^{\mathcal{T}}=\{0,1\}$ then $\operatorname{lh}_{\alpha} \leq \rho_{\beta}^{\mathcal{T}}$.

Proof. By induction. Part (i) is as for 4.3. Part (ii): If there are cofinally many $\alpha^{\prime}<\beta$ with $E_{\alpha^{\prime}}^{\mathcal{T}} \neq \emptyset$, use part (i) and rules of iteration trees. Otherwise, fix $\alpha<\beta$ least with $E_{\alpha^{\prime}}^{\mathcal{T}}=\emptyset$ for all $\alpha^{\prime} \in[\alpha, \beta)$. Note $S_{\alpha}^{\mathcal{T}}=\{0,1\}$, and if there is $\alpha^{\prime} \in[\alpha, \beta)$ with $\rho_{\alpha}^{\mathcal{T}}<\mathrm{lh}_{\alpha^{\prime}}$, and $\alpha^{\prime}$ is least such, we move into a model of $B_{\alpha^{\prime}}^{\mathcal{T}}=B_{\alpha}^{\mathcal{T}}=B_{\beta}^{\mathcal{T}}$ in $\mathcal{T}$ at stage $\alpha^{\prime}$, so $S_{\beta}^{\mathcal{T}} \neq\{0,1\}$, contradiction.

It follows as before that the comparison terminates.

Claim 2. Let $\alpha<\operatorname{lh}(\mathcal{T}, \mathcal{U})$. Then (i) the comparison does not terminate early at stage $\alpha$; (ii) if at stage $\alpha$, in $\mathcal{T}$ we move into $R$, then for every $\beta \in(\alpha, \operatorname{lh}(\mathcal{T}, \mathcal{U})$ ), we have $R \Varangle S$ for all $S \in \mathfrak{M}_{\beta}^{\mathcal{U}}$.

${ }^{24}$ That is, we also minimize on $\nu(E)$, so if $E_{\alpha}^{\mathcal{T}} \neq \emptyset \neq E_{\alpha}^{\mathcal{U}}$ then $\nu\left(E_{\alpha}^{\mathcal{T}}\right)=\nu_{\alpha}=\nu\left(E_{\alpha}^{\mathcal{U}}\right)$. 
Proof. By induction on $\alpha$. Suppose for example that Subcase 2.2 attains at stage $\alpha$. We have $P \in \mathfrak{M}_{\alpha}^{\mathcal{U}}$.

Suppose $Q^{\mathcal{T}} \unlhd P$, so in $\mathcal{T}$ we move into $M^{\mathcal{T}}$. We have $M^{\mathcal{T}}\left|\rho^{\mathcal{T}}=P\right| \rho^{\mathcal{T}}$ and $N^{\mathcal{T}} \unlhd Q^{\mathcal{T}} \unlhd P$ and $M^{\mathcal{T}} \neq N^{\mathcal{T}}$ and both $M^{\mathcal{T}}, N^{\mathcal{T}}$ project $\leq \rho^{\mathcal{T}}$ and

$$
M^{\mathcal{T}}\left\|\left(\left(\rho^{\mathcal{T}}\right)^{+}\right)^{M^{\mathcal{T}}}=N^{\mathcal{T}}\right\|\left(\left(\rho^{\mathcal{T}}\right)^{+}\right)^{N^{\mathcal{T}}}
$$

So $M^{\mathcal{T}} \not P$ and taking $\lambda$ least with $M^{\mathcal{T}}\left|\lambda \neq N^{\mathcal{T}}\right| \lambda$, we have $\rho^{\mathcal{T}}<\lambda \leq$ $\min \left(\operatorname{OR}\left(M^{\mathcal{T}}\right), \operatorname{OR}\left(N^{\mathcal{T}}\right)\right)$. So the comparison does not terminate early at stage $\alpha$, and as $M^{\mathcal{T}}$ projects $\leq \rho^{\mathcal{T}}$, for no $\beta>\alpha$ is $M^{\mathcal{T}} \triangleleft S \in \mathfrak{M}_{\beta}^{\mathcal{U}}$.

Now suppose $Q^{\mathcal{T}} \not P$, so in $\mathcal{T}$ we move into $Q^{\mathcal{T}}$. If $\alpha \notin \mathscr{B}^{\mathcal{U}}$ then $P=B^{\mathcal{U}}$ is unsound. Otherwise there is $\delta<\alpha$ such that at stage $\delta$, in $\mathcal{U}$ we move into $P$. In either case (by induction in the latter), $P \Varangle Q^{\mathcal{T}}$. So the comparison does not terminate early at stage $\alpha$. Let $\lambda$ be least with $Q^{\mathcal{T}}|\lambda \neq P| \lambda$. Then $\rho^{\mathcal{T}}<\lambda$ and since $Q^{\mathcal{T}}$ projects $\leq \gamma^{\mathcal{T}}$, there is no $\beta>\alpha$ such that $Q^{\mathcal{T}} \triangleleft S \in \mathfrak{M}_{\beta}^{\mathcal{U}}$.

The proof is similar in the remaining subcases.

Let $\alpha+1=\operatorname{lh}(\mathcal{T}, \mathcal{U})$. As in the proof of 4.3, and by Claim 2, we have $\operatorname{card}\left(S_{\alpha}^{\mathcal{T}}\right)=\operatorname{card}\left(S_{\alpha}^{\mathcal{U}}\right)=1$ and $\alpha \in \mathscr{B}^{\mathcal{T}} \Delta \mathscr{B}^{\mathcal{U}}$. We may assume $\alpha \in \mathscr{B}^{\mathcal{T}}$, so $B_{\alpha}^{\mathcal{T}}=\left(\gamma^{\prime}, \rho^{\prime}, M^{\prime}, Q^{\prime}\right)$ is a cephalanx and $B_{\alpha}^{\mathcal{U}}$ is a non-sound pm. So $P \unlhd B_{\alpha}^{\mathcal{U}}$ where $\{P\}=\mathfrak{M}_{\alpha}^{\mathcal{T}}$. But by Claim $2, P \Varangle B_{\alpha}^{\mathcal{U}}$, so $P=B_{\alpha}^{\mathcal{U}}$. Let $\beta=S^{\mathcal{T}}(\alpha)$.

Claim 3. $S_{\alpha}^{\mathcal{T}}=\{0\}$.

Proof. Suppose $S_{\alpha}^{\mathcal{T}}=\{1\}$, so $Q^{\prime}=P=B_{\alpha}^{\mathcal{U}}$ is $\gamma^{\prime}$-sound. At stage $\beta$, in $\mathcal{T}$ we move into $Q^{\prime}$. For all $\xi \in[\beta, \alpha), E_{\xi}^{\mathcal{T}}=\emptyset$, so $E_{\xi}^{\mathcal{U}} \neq \emptyset$, and $\rho^{\prime}<\mathrm{lh}_{\xi}$, because $B^{\prime}\left|\rho^{\prime}=B_{\beta}^{\mathcal{U}}\right| \rho^{\prime}$, and therefore $\rho^{\prime} \leq \nu_{\xi}^{\mathcal{U}}$, because $\rho^{\prime}$ is a cardinal of $Q^{\prime}$. But then $B_{\alpha}^{\mathcal{U}}$ is not $\gamma^{\prime}$-sound, contradicting the fact that $Q^{\prime}=B_{\alpha}^{\mathcal{U}}$.

So $M^{\prime}=P=B_{\alpha}^{\mathcal{U}}$. Let $N^{\prime}=N_{\alpha}^{\mathcal{T}}$.

Claim $4 . \operatorname{OR}\left(N^{\prime}\right)=\left(\left(\rho^{\prime}\right)^{+}\right)^{M^{\prime}}, N^{\prime}$ is active type 1 or type $3, \alpha=\beta+1$, $E_{\beta}^{\mathcal{U}}=F^{N^{\prime}}$, and if $N^{\prime}$ is type 1 then $B_{\alpha}^{* \mathcal{U}}=Q^{\prime}$.

Proof. Assume, for example, that Subcase 2.2 attains at stage $\beta$. So $N^{\prime} \unlhd Q^{\prime} \unlhd$ $B_{\beta}^{\mathcal{U}}$. We have $M^{\prime} \neq N^{\prime}$, both $M^{\prime}, N^{\prime}$ project $\leq \rho^{\prime}$, and

$$
M^{\prime}\left\|\left(\left(\rho^{\prime}\right)^{+}\right)^{M^{\prime}}=N^{\prime}\right\|\left(\left(\rho^{\prime}\right)^{+}\right)^{N^{\prime}} .
$$

We have $E_{\beta}^{\mathcal{T}}=\emptyset$, so $E_{\beta}^{\mathcal{U}} \neq \emptyset$ and note that $E_{\beta}^{\mathcal{U}} \in \mathbb{E}_{+}\left(N^{\prime}\right)$ and $\mathrm{lh}_{\beta}^{\mathcal{U}} \geq\left(\left(\rho^{\prime}\right)^{+}\right)^{M^{\prime}}$. Since $M^{\prime}=B_{\alpha}^{\mathcal{U}}$ is $\rho^{\prime}$-sound it follows that $\alpha=\beta+1$ and $\nu_{\beta}^{\mathcal{U}}=\rho^{\prime}$, so $E_{\beta}^{\mathcal{U}}$ is type 1 or type 3 . Therefore $N^{\prime} \mid \operatorname{lh}_{\beta}^{\mathcal{U}}$ projects to $\rho^{\prime}$, so $\operatorname{OR}\left(N^{\prime}\right)=\operatorname{lh}_{\beta}^{\mathcal{U}}$.

Now suppose further that $N^{\prime}$ is type 1 ; we want to see that $B_{\alpha}^{* \mathcal{U}}=Q^{\prime}$. We have $Q^{\prime} \unlhd B_{\beta}^{\mathcal{U}}$ and $\operatorname{cr}\left(F^{N^{\prime}}\right)=\gamma^{\prime}$ and $\rho_{\omega}\left(Q^{\prime}\right) \leq \gamma^{\prime}$ and

$$
\mathcal{P}\left(\gamma^{\prime}\right) \cap Q^{\prime}=\mathcal{P}\left(\gamma^{\prime}\right) \cap N^{\prime}
$$

So it suffices to see that $\operatorname{pred}^{\mathcal{U}}(\alpha)=\beta$. We may assume that $\mathrm{lh}_{\delta}^{\mathcal{U}}=\rho^{\prime}$ for some $\delta<\beta$. Then $\rho^{\prime}$ is a cardinal of $B_{\beta}^{\mathcal{U}}$, so $Q^{\prime} \nless B_{\beta}^{\mathcal{U}}$, so $Q^{\prime}=B_{\beta}^{\mathcal{U}}$. So $B_{\beta}^{\mathcal{U}}$ is 
$\gamma^{\prime}$-sound, so there is a unique $\delta$ such that $\operatorname{lh}_{\delta}^{\mathcal{U}}=\rho^{\prime}$, and moreover, $E_{\delta}^{\mathcal{U}}$ is type 3 and $\beta=\delta+1$. Therefore $\operatorname{pred}^{\mathcal{U}}(\alpha)=\beta$, as required.

To complete the proof, one can now argue like in Claim 7 of 4.3 .

4.6 Remark. We next consider active cephalanxes $B=(\gamma, \rho, M, Q)$. Here things are more subtle, for two reasons. First, if $Q$ is type 3 then $Q_{\alpha}^{\mathcal{T}}$ can fail the ISC; this complicates comparison termination. Second, if $Q$ is superstrong then comparison termination is complicated further, and more importantly, we do not see how to show $B$ has a good core (4.15), nor how to rule out the possibility that $B$ is exact and $M$ is sound with $\rho_{m+1}^{M}=\rho$. It is easy enough to illustrate how the latter might occur. Let $Q$ be a sound superstrong pm and $\kappa=\operatorname{cr}\left(F^{Q}\right)$ and $J$ be a sound pm with $J \|\left(\kappa^{++}\right)^{J}=Q \mid\left(\kappa^{++}\right)^{Q}$ and $\rho_{m+1}^{J}=\left(\kappa^{+}\right)^{Q}=\left(\kappa^{+}\right)^{J}<\rho_{m}^{J}$. Let $M=\operatorname{Ult}_{m}\left(J, F^{Q}\right)$ and $B=(\gamma, \rho, M, Q)$, where $\rho=\mathrm{OR}^{Q}$ and $\gamma=\operatorname{lgcd}(Q)$. Suppose $M$ is wellfounded. Then $B$ is an exact, sound cephalanx. (We have $\rho_{m+1}^{M}=\rho<\rho_{m}^{M}$ and $M$ is $(m+1)$-sound, and $B$ is exact because $i_{F Q}^{J, m}$ and $i_{F Q}^{Q}$ are continuous at $\left(\kappa^{++}\right)^{J}$.) It seems $J, Q$ might arise as iterates of a single mouse, so it seems $B$ might be iterable.

4.7 Definition. Let $\mathcal{T}$ be an iteration tree on an active cephalanx $B$ and $\alpha+1<\operatorname{lh}(\mathcal{T})$. We say $\alpha$ is $\mathcal{T}$-special iff $\alpha \in \mathscr{B}^{\mathcal{T}}$ and $E_{\alpha}^{\mathcal{T}}=F\left(Q_{\alpha}^{\mathcal{T}}\right)$.

4.8 Lemma. Let $\mathcal{T}$ be an iteration tree on an active cephalanx $B$ and $\alpha<$ $\operatorname{lh}(\mathcal{T})$. Then:

(a) If $\alpha \in \mathscr{B}^{\mathcal{T}}$ then $Q_{\alpha}^{\mathcal{T}}$ has superstrong type iff $Q$ does.

(b) If $\iota\left(Q^{B}\right)=\gamma^{B}$ then $\mathscr{Q}=\emptyset$.

Suppose also that $\alpha+1<\operatorname{lh}(\mathcal{T})$. Then:

(c) If $\alpha$ is $\mathcal{T}$-special then $\alpha+1 \in \mathscr{B}^{\mathcal{T}}$ and $\operatorname{pred}^{\mathcal{T}}(\alpha+1)$ is the least $\varepsilon \in[0, \alpha]_{\mathcal{T}}$ such that either $\varepsilon=\alpha$ or $\operatorname{cr}\left(F\left(Q_{\varepsilon}^{\mathcal{T}}\right)\right)<\operatorname{cr}\left(i_{\varepsilon \alpha}^{\mathcal{T}}\right)$.

(d) If $B$ is a pm-cephalanx and $\operatorname{ex}_{\alpha}^{\mathcal{T}}$ is not a premouse then $\alpha$ is $\mathcal{T}$-special (so $\left.\operatorname{ex}_{\alpha}^{\mathcal{T}}=Q_{\alpha}^{\mathcal{T}}\right)$ and $Q$ is type 3 .

Proof. For (a), recall that in $\mathcal{T}$, we only form simple ultrapowers of $Q^{B}$ and its images.

4.9 Lemma. Let $\mathcal{T}$ be an iteration tree on an active pm-cephalanx $B=(\gamma, \rho, M, Q)$. Let $\alpha<\beta<\operatorname{lh}(\mathcal{T})$. Let $\lambda=\operatorname{lh}_{\alpha}^{\mathcal{T}}$. Then either:

1. $\beta \notin \mathscr{B}^{\mathcal{T}}$ and either $(i) \lambda<\operatorname{OR}\left(B_{\beta}^{\mathcal{T}}\right)$ and $\lambda$ is a cardinal of $B_{\beta}^{\mathcal{T}}$, or $($ ii $)$ $\beta=\alpha+1, E_{\alpha}^{\mathcal{T}}$ has superstrong type, $\lambda=\mathrm{OR}\left(B_{\beta}^{\mathcal{T}}\right)$ and $B_{\beta}^{\mathcal{T}}$ is an active type 2 premouse; or

2. $\beta \in \mathscr{B}^{\mathcal{T}}$ and either $(i) \lambda<\rho\left(B_{\beta}^{\mathcal{T}}\right)$ and $\lambda$ is a cardinal of $B_{\beta}^{\mathcal{T}}$, or $($ ii) $\beta=\alpha+1, E_{\alpha}^{\mathcal{T}}$ has superstrong type, $\lambda=\rho\left(B_{\beta}^{\mathcal{T}}\right)$, and letting $\varepsilon=\operatorname{pred}^{\mathcal{T}}(\beta)$, $\operatorname{cr}_{\alpha}^{\mathcal{T}}=\gamma\left(B_{\varepsilon}^{\mathcal{T}}\right)$. 
Therefore if $\operatorname{lh}_{\alpha}^{\mathcal{T}}<\operatorname{lh}_{\beta}^{\mathcal{T}}$ then $\operatorname{lh}_{\alpha}^{\mathcal{T}}$ is a cardinal of $\operatorname{ex}_{\beta}^{\mathcal{T}}$.

Proof. If $\beta=\alpha+1$ it is straightforward to prove the conclusion. Now suppose $\beta>\alpha+1$. If $\lambda<\operatorname{lh}_{\alpha+1}^{\mathcal{T}}$ it is straightforward, so suppose $\lambda=\operatorname{lh}_{\alpha+1}^{\mathcal{T}}$. Then since the lemma held for $\beta=\alpha+1$, either $E_{\alpha+1}^{\mathcal{T}}$ is type 2 , in which case things are straightforward, or $\alpha+1$ is $\mathcal{T}$-special, so letting $\mu=\operatorname{cr}_{\alpha+1}^{\mathcal{T}}$ and $\chi=\operatorname{pred}^{\mathcal{T}}(\alpha+2)$, we have that $B_{\alpha+2}^{* \mathcal{T}}=B_{\chi}^{\mathcal{T}}$ is a cephalanx and $\mu<\gamma\left(B_{\chi}^{\mathcal{T}}\right)$, which implies that $\lambda<\rho\left(B_{\alpha+2}^{\mathcal{T}}\right)$ and $\lambda$ is a cardinal of $B_{\alpha+2}^{\mathcal{T}}$. The rest is clear.

4.10 Definition. Let $B=(\gamma, \rho, M, Q)$ be an active cephalanx of degree $(m, 0)$. We say that $B$ is exceptional iff (i) $B$ is exact, (ii) $Q$ has superstrong type, and (iii) either $\rho_{m+1}^{M}=\rho$ or $M$ is not $\gamma$-sound.

4.11 Lemma. Let $M$ be an $m$-sound premouse and let $\rho_{m+1}^{M} \leq \gamma<\rho_{m}^{M}$. Then $M$ is $\gamma$-sound iff $M=\operatorname{Hull}_{m+1}^{M}\left(\gamma \cup z_{m+1}^{M} \cup \vec{p}_{m}^{M}\right)$.

Proof. This follows from [12, 2.17].

4.12 Lemma. Let $B, B^{\prime}$ be active cephalanxes such that $B^{\prime}$ is an iterate of $B$. Then $B^{\prime}$ is exceptional iff $B$ is exceptional.

Proof. By 3.26, 4.8(a) and 4.11 and [12, 2.20].

4.13 Definition. Let $B=(\gamma, \rho, M, Q)$ be an active cephalanx of degree $(m, 0)$. Then $B$ has an exceptional core iff $Q$ has superstrong type and the following holds. Let $F=F^{Q}, \kappa=\operatorname{cr}(F), X=i_{F}^{Q} "\left(\kappa^{+}\right)^{Q}, m^{\prime}=\max (m, 0)$,

$$
H=\mathrm{cHull}_{m^{\prime}+1}^{M}\left(X \cup z_{m+1}^{M} \cup \vec{p}_{m}^{M}\right),
$$

$\pi: H \rightarrow M$ the uncollapse. Then $\pi^{\text {" }}\left(\kappa^{+}\right)^{H}=X$ and $H \|\left(\kappa^{++}\right)^{H}=M \mid\left(\kappa^{++}\right)^{M}$.

4.14 Lemma. Let $B=(\gamma, \rho, M, Q)$ be an active pm-cephalanx of degree $(m, 0)$. Suppose $B$ has an exceptional core. Let $F, \kappa, m^{\prime}, H, \pi$ be as in 4.13. Then:

1. $M=\mathrm{Ult}_{m^{\prime}}(H, F)$ and $\pi=i_{F}^{H, m^{\prime}}$ is an $m^{\prime}$-embedding.

2. $\pi\left(z_{m+1}^{H}\right)=z_{m+1}^{M}$ and $\pi\left(p_{m+1}^{H} \backslash\left(\kappa^{+}\right)^{H}\right)=p_{m+1}^{M} \backslash \rho$.

3. $\rho_{m+1}^{H} \leq\left(\kappa^{+}\right)^{H}<\rho_{m}^{H}$ and $H$ is $\left(\kappa^{+}\right)^{H}$-sound.

4. If $\rho_{m+1}^{H}=\left(\kappa^{+}\right)^{H}$ then $\rho_{m+1}^{M}=\rho$ and $H, M$ are $(m+1)$-sound.

5. If $\rho_{m+1}^{H} \leq \kappa$ then $\rho_{m+1}^{H}=\rho_{m+1}^{M}$ and $M$ is not $(m+1)$-sound.

6. If $M=\operatorname{Hull}_{m^{\prime}+1}^{M}\left(\alpha \cup z_{m+1}^{M} \cup \vec{p}_{m}^{M}\right)$ where $\alpha<\rho$ and $\alpha$ is least such, then $\alpha \in \operatorname{rg}(\pi)$. 
Proof. Part 1: If $m^{\prime}=-1$ this is easy, so suppose $m=m^{\prime} \geq 0$. The uncollapse map $\pi: H \rightarrow M$ is a near $m$-embedding. Let $\pi(\bar{z})=z_{m+1}^{M}$. We have

$$
H=\operatorname{Hull}_{m+1}^{H}\left(\left(\kappa^{+}\right)^{H} \cup\left\{\bar{z}, \vec{p}_{m}^{H}\right\}\right),
$$

and $H \|\left(\kappa^{++}\right)^{H}=M \mid\left(\kappa^{++}\right)^{M}$, so $H$ collapses $\left(\kappa^{++}\right)^{M}$ and $H \notin M$. It follows that $\pi$ is an $m$-embedding. Now

$$
M=\operatorname{Hull}_{m+1}^{M}\left(\gamma^{+M} \cup\left\{z_{m+1}^{M}, \vec{p}_{m}^{M}\right\}\right),
$$

Let $M^{\prime}=\operatorname{Ult}_{m}(H, F)$ and $\pi^{\prime}=i_{F}^{H, m}$ and $\sigma: M^{\prime} \rightarrow M$ the natural factor map. Then $\pi^{\prime}$ is an $m$-embedding and $\sigma$ is $m$-lifting and $\sigma \circ \pi^{\prime}=\pi$, and since $\pi$ is an $m$-embedding, in fact so is $\sigma$. But $\operatorname{cr}(\sigma) \geq \operatorname{lh}(F)=\gamma^{+M}$ and $z_{m+1}^{M} \in \operatorname{rg}(\sigma)$, so by line (4), $M^{\prime}=M$ and $\sigma=\mathrm{id}$.

Parts 2-4: If $m=-1$ this is trivial, so suppose $m=m^{\prime} \geq 0$. By [12] and part $1, \bar{z}=z_{m+1}^{H}$ (where $\pi(\bar{z})=z_{m+1}^{M}$ ), so by 4.11 and line (3) above, $H$ is $\left(\kappa^{+}\right)^{H}$-sound and $p_{m+1}^{H} \backslash\left(\kappa^{+}\right)^{H}=\bar{z} \backslash\left(\kappa^{+}\right)^{H}$, so

$$
\pi\left(p_{m+1}^{H} \backslash\left(\kappa^{+}\right)^{H}\right)=z_{m+1}^{M} \backslash \rho=p_{m+1}^{M} \backslash \rho,
$$

since $M$ is $\rho$-sound. The rest follows from [12].

Part 5: Because $\rho_{m+1}^{H} \leq \kappa$, we have $m \geq 0$. Since $Q$ is a type 3 premouse (as $B$ is a pm-cephalanx) and $M\left\|\left(\kappa^{++}\right)^{M}=H\right\|\left(\kappa^{++}\right)^{H}, F$ is close to $H$, so $\rho_{m+1}^{M}=\rho_{m+1}^{H} \leq \kappa$. Suppose $M$ is $(m+1)$-sound, so $M=\operatorname{Hull}_{m+1}^{M}\left(\kappa \cup \vec{p}_{m+1}^{M}\right)$. Then $M=\operatorname{Hull}_{m+1}^{M}(\operatorname{rg}(\pi) \cup q)$ some $q \in \gamma^{<\omega}$. But the generators of $F$ are unbounded in $\gamma$, a contradiction.

Part 6: Suppose there is $\alpha<\rho$ such that

$$
M=\operatorname{Hull}_{m^{\prime}+1}^{M}\left(\alpha \cup z_{m+1}^{M} \cup \vec{p}_{m}^{M}\right) .
$$

Let $\alpha$ be least such. Note that $\alpha \geq \gamma$, since $Q$ is a premouse and $F$ has superstrong type. Now if $\alpha>\gamma$ then $\alpha$ is a successor. For if not, then since $\alpha<\rho$, there is a surjection $f: \gamma \rightarrow \alpha$ in $M$, so there is $\xi<\alpha$ with

$$
f \in \operatorname{Hull}_{m^{\prime}+1}^{M}\left(\left\{\xi, z_{m+1}^{M}, \vec{p}_{m}^{M}\right\}\right),
$$

but then $\max (\xi+1, \gamma)<\alpha$ suffices in place of $\alpha$, a contradiction.

Since $\pi$ is cofinal in $\rho=\gamma^{+M}$ and $\pi$ is $\mathrm{r} \Sigma_{m^{\prime}+1^{-}}$-elementary and $\pi\left(z_{m+1}^{H}\right)=$ $z_{m+1}^{M}$, the existence of $\alpha$ reflects to $H$, in that there is $\beta<\left(\kappa^{+}\right)^{H}$ such that

$$
H=\operatorname{Hull}_{m^{\prime}+1}^{H}\left(\beta \cup z_{m+1}^{H} \cup \vec{p}_{m}^{H}\right) .
$$

Let $\beta$ be least such. As above, either $\beta \leq \kappa$ or $\beta=\zeta+1$ for some $\zeta$. If $\beta \leq \kappa$ then note $\alpha=\gamma=\pi(\kappa)$. So suppose $\beta=\zeta+1>\kappa$. We claim $\pi(\beta)=\alpha$. For

$$
\zeta \notin \operatorname{Hull}_{m^{\prime}+1}^{H}\left(\zeta \cup z_{m+1}^{H} \cup \vec{p}_{m}^{H}\right),
$$

and this non-membership is an $\mathrm{r}_{m^{\prime}+1}$ assertion in these parameters, which therefore lifts to $M$ and $\pi(\zeta)$, etc, so $\pi(\beta) \leq \alpha$. Conversely, because

$$
H=\operatorname{Hull}_{m^{\prime}+1}^{H}\left((\zeta+1) \cup z_{m+1}^{H} \cup \vec{p}_{m}^{H}\right),
$$


we get $\pi^{\prime \prime} H=\operatorname{Hull}_{m^{\prime}+1}^{M}\left(\pi^{\prime \prime}(\zeta+1) \cup z_{m+1}^{M} \cup \vec{p}_{m}^{M}\right)$ is unbounded in $\rho=\gamma^{+M}$, but $\gamma \leq \pi(\zeta)$, so

$$
\gamma^{+M} \subseteq \operatorname{Hull}_{m^{\prime}+1}^{M}\left(\pi(\zeta+1) \cup z_{m+1}^{M} \cup \vec{p}_{m}^{M}\right),
$$

so $\pi(\beta) \geq \alpha$.

4.15 Definition. Let $B=(\gamma, \rho, M, Q)$ be an active cephalanx. We say $B$ has a good core iff the following statements $((a)-(c))$ hold:

(a) $B$ has degree $(m, 0)$ with $m \geq 0$.

(b) Either

(i) $B$ is exact, and let $F=F^{Q}$; or

(ii) $B$ is non-exact, and letting $N=N^{B}$, we have $\mathrm{OR}^{N}=\rho^{+M}$ and $N$ is active type 1 or 3 ; let $F=F^{N}$.

(c) Let $\kappa=\operatorname{cr}(F)$ and $\nu=\nu(F)$. Then

(i) $M$ has an $(m, \nu)$-good core at $\kappa$, and $G_{m, \kappa, \nu}^{M}=F\lceil\nu$.

(ii) Suppose case (ii) above holds and $N$ is type 1 ; so $\kappa=\gamma$. Then:

- If $Q$ is type 2 then $H_{m, \kappa}^{M}=Q$.

- Suppose $Q$ is not type 2, nor superstrong. Let $\mu=\operatorname{cr}\left(F^{Q}\right)$. Then $M$ has an $(m, \gamma)$-good core at $\mu$, and $G_{m, \mu, \gamma}^{M}=F^{Q}$.

4.16 Remark. It seems that $B$ might have an exceptional non-good core.

4.17 Theorem. Let $B=(\gamma, \rho, M, Q)$ be an $\left(\omega_{1}+1\right)$-iterable, non-trivial, active pm-cephalanx, of degree $(m, q)$. Then:

- If $B$ is exceptional then $B$ has an exceptional core (see 4.13, 4.14).

- If $B$ is non-exceptional then $m \geq 0$ and $B$ has a good core (see 4.15), so $B$ is not sound (i.e. $M$ is not $(m+1)$-sound).

Proof. Suppose first that $B$ is exact and $Q$ is superstrong, but $B$ is not exceptional. Then $\rho_{m+1}^{M} \leq \gamma \leq\left(\gamma^{++}\right)^{M}=\rho^{+M} \leq \rho_{m}^{M}$ and $M$ is $\gamma$-sound, as is $Q$. Note then that $m \geq 0$, since otherwise $\mathrm{OR}^{M}=\rho_{-1}^{M}=\rho_{m}^{M}=\left(\left(\rho_{m+1}^{M}\right)^{+}\right)^{M}$. So $C=(\gamma, M, Q)$ is a non-trivial bicephalus, and note that $C$ is $\left(\omega_{1}+1\right)$-iterable. So by $4.3, M$ has an $(m, \gamma)$-good core corresponding to $F^{Q}$, and because $B$ is exact, this implies that $B$ has a good core (4.15). So we may now assume that:

If $B$ is exact and $Q$ is superstrong, then $B$ is exceptional.

Under this assumption, the proof is based on that of 4.5. The main differences occur in the rules guiding the comparison, the proof that the comparison terminates, and when $B$ is exceptional. We may assume $B$ is countable.

We define $\mathcal{T}, \mathcal{U}$ on $B$ and sets $S_{\alpha}^{\mathcal{T}}, S_{\alpha}^{\mathcal{U}}, \mathfrak{M}_{\alpha}^{\mathcal{T}}, \mathfrak{M}_{\alpha}^{\mathcal{U}}$, much as before. (But if $Q$ is type 1 or 3 then $S_{\alpha}^{\mathcal{T}} \neq\{1\} \neq S_{\alpha}^{\mathcal{U}}$ for all $\alpha$.) If $S_{\alpha}^{\mathcal{T}}=\{0,1\}$ and $B_{\alpha+1}^{\mathcal{T}}=B_{\alpha}^{\mathcal{T}}$ 
and $S_{\alpha+1}^{\mathcal{T}}=\{0\}$, we say that (in $\mathcal{T}$ ) we move into $M_{\alpha}^{\mathcal{T}}$ at stage $\alpha$. We never move into $Q_{\alpha}^{\mathcal{T}}$; that is, if $\alpha \in \mathscr{B}^{\mathcal{T}}$ then $S_{\alpha}^{\mathcal{T}} \neq\{1\}$. Likewise for $\mathcal{U}$. Also, we allow $\alpha$ such that $E_{\alpha}^{\mathcal{T}}=\emptyset=E_{\alpha}^{\mathcal{U}}$, but only when we move into $M_{\alpha}^{\mathcal{T}}$ or $M_{\alpha}^{\mathcal{U}}$ at stage $\alpha$. If we move into $M_{\alpha}^{\mathcal{T}}$ in at stage $\alpha$, we will have $E_{\alpha}^{\mathcal{T}}=\emptyset$ and either $E_{\alpha}^{\mathcal{U}}=\emptyset$ or $E_{\alpha}^{\mathcal{U}}=F\left(Q_{\alpha}^{\mathcal{T}}\right)$. Likewise for $\mathcal{U}$. We will not move into both $M_{\alpha}^{\mathcal{T}}$ (in $\mathcal{T}$ ) and $M_{\alpha}^{\mathcal{U}}$ (in $\mathcal{U}$ ) at stage $\alpha$. If $\alpha \in \mathscr{B}^{\mathcal{T}}$ and $\ln \left(E_{\alpha}^{\mathcal{T}}\right)>\rho\left(B_{\alpha}^{\mathcal{T}}\right)$ (that is, $\left.E_{\alpha}^{\mathcal{T}} \in \mathbb{E}_{+}\left(M_{\alpha}^{\mathcal{T}}\right) \backslash \mathbb{E}_{+}\left(Q_{\alpha}^{\mathcal{T}}\right)\right)$, then we will have $S_{\alpha}^{\mathcal{T}}=\{0\}$, so there is some $\beta<\alpha$ such that $B_{\beta}^{\mathcal{T}}=B_{\alpha}^{\mathcal{T}}$ and at stage $\beta$ we move into $M_{\beta}^{\mathcal{T}}=M_{\alpha}^{\mathcal{T}}$. Likewise for $\mathcal{U}$.

Suppose we have defined $(\mathcal{T}, \mathcal{U}) \uparrow \alpha+1, S_{\alpha}^{\mathcal{T}}$ and $S_{\alpha}^{\mathcal{U}}$. Suppose there are $A \in \mathfrak{M}_{\alpha}^{\mathcal{T}}$ and $B \in \mathfrak{M}_{\alpha}^{\mathcal{U}}$ such that $A \nsupseteq B \nsupseteq A$; otherwise the comparison terminates at stage $\alpha$. We next determine what to do at stage $\alpha$. In certain cases we implicitly specify two segmented-premice $A, B$, with $A \unlhd B \nsubseteq A$, from which to select $E_{\alpha}^{\mathcal{T}}, E_{\alpha}^{\mathcal{U}}$. We then find the least disagreement between $A, B$, and then minimize on $\iota(E)$, rather than $\nu(E)$, when selecting extenders. (For example, if $E_{\alpha}^{\mathcal{T}} \neq \emptyset \neq E_{\alpha}^{\mathcal{U}}$ then $\iota_{\alpha}^{\mathcal{T}}=\iota_{\alpha}^{\mathcal{U}}$.)

Let $B^{\mathcal{T}}=B_{\alpha}^{\mathcal{T}}, M^{\mathcal{T}}=M_{\alpha}^{\mathcal{T}}$, etc. If $S^{\mathcal{T}}=\{0,1\}$, then we will have by induction that $(\dagger)$ for every $\beta<\alpha$, if $E_{\beta}^{\mathcal{T}} \neq \emptyset$ then $\operatorname{lh}_{\beta}^{\mathcal{T}} \leq \rho^{\mathcal{T}}$ and $\iota_{\beta}^{\mathcal{T}} \leq \gamma^{\mathcal{T}}$, and if $E_{\beta}^{\mathcal{U}} \neq \emptyset$ then $\operatorname{lh}_{\beta}^{\mathcal{U}} \leq \rho^{\mathcal{T}}$. Likewise regarding $\rho^{\mathcal{U}}, \gamma^{\mathcal{U}}$ if $S^{\mathcal{U}}=\{0,1\}$. We leave the maintenance of $(\dagger)$ to the reader.

We say that $\alpha$ is $(\mathcal{T}, \mathcal{U})$-unusual iff $S^{\mathcal{T}}=\{0,1\}$ and either

(i) there is $\xi<\alpha$ such that $F\left(Q^{\mathcal{T}}\right)\left\lceil\nu\left(F\left(Q^{\mathcal{T}}\right)\right)=E_{\xi}^{\mathcal{U}}\left\lceil\nu_{\xi}^{\mathcal{U}}\right.\right.$, or

(ii) there are $\xi_{0}<\xi_{1}<\alpha$ such that

$$
\begin{aligned}
& -\alpha=\xi_{1}+1, \\
& -S_{\xi_{0}}^{\mathcal{T}}=\{0,1\} \text { and } E_{\xi_{0}}^{\mathcal{T}}=\emptyset \text { and } E_{\xi_{0}}^{\mathcal{U}}=F\left(Q_{\xi_{0}}^{\mathcal{T}}\right) \text { and } S_{\xi_{0}+1}^{\mathcal{T}}=\{0\}, \\
& -S_{\xi_{1}}^{\mathcal{U}}=\{0,1\} \text { and } E_{\xi_{1}}^{\mathcal{U}}=\emptyset \text { and } E_{\xi_{1}}^{\mathcal{T}}=F\left(Q_{\xi_{1}}^{\mathcal{U}}\right) \text { and } S_{\xi_{1}+1}^{\mathcal{U}}=\{0\}, \\
& -\operatorname{cr}_{\xi_{1}}^{\mathcal{T}}=\gamma\left(B_{\xi_{0}}^{\mathcal{T}}\right) .
\end{aligned}
$$

In case $(\mathrm{i}) /(\mathrm{ii})$ we say that $\alpha$ is type $(\mathrm{i}) /(\mathrm{ii})$. We define $(\mathcal{U}, \mathcal{T})$-unusual symmetrically.

CAse 1. $\alpha$ is both $(\mathcal{T}, \mathcal{U})$ - and $(\mathcal{U}, \mathcal{T})$-unusual.

We terminate the comparison here with unusual failure 1 . (We will show that this can not occur.)

From now on, we assume that $\alpha$ is not both $(\mathcal{T}, \mathcal{U})$ - and $(\mathcal{U}, \mathcal{T})$-unusual. CAsE 2. Either:

$$
\begin{aligned}
& -\operatorname{card}\left(S^{\mathcal{T}}\right)=\operatorname{card}\left(S^{\mathcal{U}}\right)=1, \text { or } \\
& -S^{\mathcal{T}}=\{0,1\} \text { and } B^{\mathcal{T}}\left\|\rho^{\mathcal{T}} \neq B^{\mathcal{U}}\right\| \rho^{\mathcal{T}} \text { and if } S^{\mathcal{U}}=\{0,1\} \text { then } \rho^{\mathcal{T}} \leq \rho^{\mathcal{U}}, \text { or } \\
& -S^{\mathcal{U}}=\{0,1\} \text { and } B^{\mathcal{U}}\left\|\rho^{\mathcal{U}} \neq B^{\mathcal{T}}\right\| \rho^{\mathcal{U}} \text { and if } S^{\mathcal{T}}=\{0,1\} \text { then } \rho^{\mathcal{U}} \leq \rho^{\mathcal{T}} .
\end{aligned}
$$

Select extenders by least disagreement and minimization on $\iota(E)$ (there is no moving into models). 
Case 3. $S^{\mathcal{T}}=\{0,1\}, B^{\mathcal{T}}\left\|\rho^{\mathcal{T}}=B^{\mathcal{U}}\right\| \rho^{\mathcal{T}}$ and if $S^{\mathcal{U}}=\{0,1\}$ then $\rho^{\mathcal{T}}<\rho^{\mathcal{U}}$.

SubCASE 3.1. $\alpha$ is $(\mathcal{T}, \mathcal{U})$-unusual (hence not $(\mathcal{U}, \mathcal{T})$-unusual).

If $B^{\mathcal{U}} \mid \rho^{\mathcal{T}}$ is active, we terminate with unusual failure $\mathbf{2}$ (we will show this cannot occur). Otherwise, in $\mathcal{T}$ we move into $M^{\mathcal{T}}$, and we set $E_{\alpha}^{\mathcal{T}}=\emptyset=E_{\alpha}^{\mathcal{U}}$ and $S_{\alpha+1}^{\mathcal{U}}=S_{\alpha}^{\mathcal{U}}$.

SubCASE 3.2. $\alpha$ is $(\mathcal{U}, \mathcal{T})$-unusual (hence not $(\mathcal{T}, \mathcal{U})$-unusual).

We terminate with unusual failure 3. (We will show this cannot occur.)

SubCASE 3.3. $\alpha$ is neither $(\mathcal{T}, \mathcal{U})$ - nor $(\mathcal{U}, \mathcal{T})$-unusual.

If $Q^{\mathcal{T}} \unlhd B^{\mathcal{U}}$ then in $\mathcal{T}$ we move into $M^{\mathcal{T}}$, and we set $E_{\alpha}^{\mathcal{U}}=F\left(Q^{\mathcal{T}}\right)$.

If $Q^{\mathcal{T}} \not B^{\mathcal{U}}$ then we select extenders from $Q^{\mathcal{T}}$ and $B^{\mathcal{U}} .{ }^{25}$

CASE 4. $S^{\mathcal{U}}=\{0,1\}, B^{\mathcal{U}}\left\|\rho^{\mathcal{U}}=B^{\mathcal{T}}\right\| \rho^{\mathcal{U}}$ and if $S^{\mathcal{T}}=\{0,1\}$ then $\rho^{\mathcal{U}}<\rho^{\mathcal{T}}$.

We have subcases 4.1-4.3 analogous to 3.1-3.3.

CASE 5. $S^{\mathcal{U}}=\{0,1\}=S^{\mathcal{T}}$, and $B^{\mathcal{T}}\left\|\rho^{\mathcal{T}}=B^{\mathcal{U}}\right\| \rho^{\mathcal{U}}$ (so $\rho^{\mathcal{T}}=\rho^{\mathcal{U}}$.

SubCase 5.1. $\alpha$ is either $(\mathcal{T}, \mathcal{U})$-unusual or $(\mathcal{U}, \mathcal{T})$-unusual.

We terminate with unusual failure 4 . (We will show this cannot occur.)

SUBCASE 5.2. Otherwise.

If $Q^{\mathcal{T}} \neq Q^{\mathcal{U}}$, we select extenders from $Q^{\mathcal{T}}$ and $Q^{\mathcal{U}} \cdot{ }^{26}$

If $Q^{\mathcal{T}}=Q^{\mathcal{U}}$, either

- in $\mathcal{T}$ we move into $M^{\mathcal{T}}$, and set $E_{\alpha}^{\mathcal{U}}=F\left(Q^{\mathcal{T}}\right)=F\left(Q^{\mathcal{U}}\right)$, or

- in $\mathcal{U}$ we move into $M^{\mathcal{U}}$, and set $E_{\alpha}^{\mathcal{T}}=F\left(Q^{\mathcal{T}}\right)=F\left(Q^{\mathcal{U}}\right) .{ }^{27}$

This completes the rules of comparison. Given $\alpha<\operatorname{lh}(\mathcal{T}, \mathcal{U})$ such that $\alpha \in \mathscr{B}^{\mathcal{T}}$ but $S_{\alpha}^{\mathcal{T}}=\{0\}$, we set movin ${ }^{\mathcal{T}}(\alpha)=$ the $\beta \leq \mathcal{T} \alpha$ such that $B_{\alpha}^{\mathcal{T}}=B_{\beta}^{\mathcal{T}}$ and at stage $\beta$, in $\mathcal{T}$ we move into $M_{\beta}^{\mathcal{T}}=M_{\alpha}^{\mathcal{T}}$. Likewise for movin ${ }^{\mathcal{U}}$.

By Claim 1(7) below, the comparison does not terminate unusually. By Claim 1(4), no two extenders used in $\mathcal{T}$ and $\mathcal{U}$ are equivalent to each other. If $B$ is active and $Q$ type 3 then $Q_{\alpha}^{\mathcal{T}}$ might fail the ISC, so this needs an argument.

Claim 1. Let $\eta \leq \operatorname{lh}(\mathcal{T}, \mathcal{U})$. Then:

1. If $Q$ is type $1 / 3$ then $S_{\alpha}^{\mathcal{T}} \neq\{1\} \neq S_{\alpha}^{\mathcal{U}}$ for all $\alpha<\eta$.

2. If $Q$ is type $1 / 2$ then for every $\alpha<\eta, M_{\alpha}^{\mathcal{T}}, Q_{\alpha}^{\mathcal{T}}$ are premice (or empty).

3. For all $\alpha+1<\eta$ :

\footnotetext{
${ }^{25}$ It might be that $B^{\mathcal{U}} \mid \mathrm{OR}\left(Q^{\mathcal{T}}\right)$ is active with extender $E$ and $\iota\left(F\left(Q^{\mathcal{T}}\right)\right)>\iota(E)$, in which case $E_{\alpha}^{\mathcal{T}}=\emptyset$ and $E_{\alpha}^{\mathcal{U}}=E$. In this case we keep $S_{\alpha+1}^{\mathcal{T}}=\{0,1\}$. If $E$ is superstrong, we could end up with $F\left(Q^{\mathcal{T}}\right)$ active on some $S \in \mathfrak{M}_{\alpha+1}^{\mathcal{U}}$.

${ }^{26}$ Here it would have been equivalent to set $E_{\alpha}^{\mathcal{T}}=F\left(Q^{\mathcal{T}}\right)$ and $E_{\alpha}^{\mathcal{U}}=F\left(Q^{\mathcal{U}}\right)$. We don't do this because if $Q$ is type 2 , it seems it might break the rule that we minimize on $\iota(E)$ before selecting extenders - albeit in a trivial manner. (Suppose $Q$ is type 2. Then so are $Q^{\mathcal{T}}$ and $Q^{\mathcal{U}}$. Suppose $\iota\left(Q^{\mathcal{T}}\right)=\nu\left(Q^{\mathcal{T}}\right)<\nu\left(Q^{\mathcal{U}}\right)=\iota\left(Q^{\mathcal{U}}\right)$, so $E_{\alpha}^{\mathcal{T}}=F\left(Q^{\mathcal{T}}\right)$ and $E_{\alpha}^{\mathcal{U}}=\emptyset$ and $S_{\alpha+1}^{\mathcal{U}}=\{0,1\}$. Since $Q^{\mathcal{T}}$ is type 2 , we have $B_{\alpha+1}^{\mathcal{T}} \mid \mathrm{OR}\left(Q^{\mathcal{U}}\right)$ is well-defined and is passive, so we end up with $E_{\alpha}^{\mathcal{T}}=\emptyset$ and $E_{\alpha+1}^{\mathcal{U}}=F\left(Q^{\mathcal{U}}\right)$.)

${ }^{27}$ We choose a side randomly. We could have specified one, but at a loss of symmetry.
} 
(a) $E_{\alpha}^{\mathcal{T}}=\emptyset=E_{\alpha}^{\mathcal{U}} \Longrightarrow[\alpha$ is either $(\mathcal{T}, \mathcal{U})$ - or $(\mathcal{U}, \mathcal{T})$-unusual $]$.

(b) $E_{\alpha}^{\mathcal{T}}=\emptyset=E_{\alpha}^{\mathcal{U}} \Longleftarrow[\alpha$ is either $(\mathcal{T}, \mathcal{U})$ - or $(\mathcal{U}, \mathcal{T})$-unusual $]$.

(c) If we move into $M_{\alpha}^{\mathcal{T}}$ at stage $\alpha$ then $B_{\alpha}^{\mathcal{T}}\left\|\rho_{\alpha}^{\mathcal{T}}=B_{\alpha}^{\mathcal{U}}\right\| \rho_{\alpha}^{\mathcal{T}}$ and $E_{\alpha}^{\mathcal{T}}=\emptyset$ and [either $E_{\alpha}^{\mathcal{U}}=\emptyset$ or $\left.E_{\alpha}^{\mathcal{U}}=F\left(Q_{\alpha}^{\mathcal{T}}\right)\right]$ and if $S_{\alpha}^{\mathcal{U}}=\{0,1\}$ then $\rho_{\alpha}^{\mathcal{T}} \leq \rho_{\alpha}^{\mathcal{U}}$.

(d) If $\alpha \in \mathscr{B}^{\mathcal{T}}$ and $E_{\alpha}^{\mathcal{T}} \in \mathbb{E}_{+}\left(M_{\alpha}^{\mathcal{T}}\right) \backslash \mathbb{E}_{+}\left(Q_{\alpha}^{\mathcal{T}}\right)$ then $S_{\alpha}^{\mathcal{T}}=\{0\}$.

4. For all $\alpha+1, \beta+1<\eta$, if $E_{\alpha}^{\mathcal{T}} \neq \emptyset \neq E_{\beta}^{\mathcal{U}}$ then $E_{\alpha}^{\mathcal{T}}\left\lceil\nu_{\alpha}^{\mathcal{T}} \neq E_{\beta}^{\mathcal{U}}\left\lceil\nu_{\beta}^{\mathcal{U}}\right.\right.$.

5. Let $\alpha<\eta$ be $(\mathcal{T}, \mathcal{U})$-unusual. Then:

(i) $Q$ is type 3 and $Q_{\alpha}^{\mathcal{T}}$ is not a premouse.

(ii) $\alpha$ is not $(\mathcal{U}, \mathcal{T})$-unusual.

(iii) Case 3 of the comparison rules attains at stage $\alpha$ and $B_{\alpha}^{\mathcal{T}} \| \rho_{\alpha}^{\mathcal{T}}=$ $B_{\alpha}^{\mathcal{U}} \mid \rho_{\alpha}^{\mathcal{T}}$ is passive.

(iv) For all $\beta \in[\alpha, \eta)$, if $\beta \in \mathscr{B}^{\mathcal{T}}$ then $\operatorname{cr}\left(F\left(Q_{\beta}^{\mathcal{T}}\right)\right) \neq \gamma_{\alpha}^{\mathcal{T}}$, and if $\beta \in \mathscr{B}^{\mathcal{U}}$ then $\operatorname{cr}\left(F\left(Q_{\beta}^{\mathcal{U}}\right)\right) \neq \gamma_{\alpha}^{\mathcal{T}}$.

(v) Suppose $\alpha$ is type (i), as witnessed by $\xi$. Then:

(a) $Q$ is not superstrong,

(b) $E_{\xi}^{\mathcal{T}}=\emptyset$ and $\alpha=\xi+1$,

(c) $\operatorname{lh}_{\xi}^{\mathcal{U}}<\gamma_{\alpha}^{\mathcal{T}}$,

(d) the trivial completion of $E_{\xi}^{\mathcal{U}}\left\lceil\nu_{\xi}^{\mathcal{U}}\right.$ is a type 3 premouse extender, ${ }^{28}$

(vi) Suppose that $\alpha$ is type (ii), as witnessed by $\xi_{0}, \xi_{1}$. Then:

(a) $Q$ is superstrong,

(b) $B$ is exact,

(c) $M_{\alpha}^{* \mathcal{T}}=B_{\xi_{0}}^{\mathcal{T}}$,

(d) $F\left(Q_{\alpha}^{\mathcal{T}}\right)=E_{\xi_{1}}^{\mathcal{T}} \circ E_{\xi_{0}}^{\mathcal{U}}$.

(e) $M_{\alpha}^{\mathcal{T}}\left\|\left(\left(\rho_{\alpha}^{\mathcal{T}}\right)^{+}\right)^{M_{\alpha}^{\mathcal{T}}}=M_{\alpha}^{\mathcal{U}}\right\|\left(\left(\rho_{\alpha}^{\mathcal{T}}\right)^{+}\right)^{M_{\alpha}^{\mathcal{U}}}$.

6. Likewise for $(\mathcal{U}, \mathcal{T})$-unusual $\alpha<\eta$.

7. The comparison does not terminate unusually at any stage $\alpha<\eta$.

Proof. We prove all parts together by simultaneous induction on $\eta$.

Parts 1, 2, 3a, 3c, 3d: by the rules of comparison and for normal trees.

Part 4: Suppose otherwise. Then by part 2 and the rules of comparison, $Q$ is type 3 , so part 1 applies. Let $(\alpha, \beta)$ be the lexicographically least counterexample (with $\alpha+1, \beta+1<\eta$ ). Let $\lambda=\operatorname{lh}_{\alpha}^{\mathcal{T}}$.

Suppose that $\mathrm{lh}_{\beta}^{\mathcal{U}}=\lambda$. So $E_{\alpha}^{\mathcal{T}}=E_{\beta}^{\mathcal{U}}$, so by the rules of extender selection, $\alpha \neq \beta$. So suppose $\alpha<\beta$ (the other case is almost symmetric). The rules give some $\delta \in[\alpha, \beta)$ with $E_{\delta}^{\mathcal{U}} \neq \emptyset$; let $\delta$ be least such and let $G=E_{\delta}^{\mathcal{U}}$. Then

${ }^{28}$ Recall that a premouse extender is the active extender of some premouse. 
$\operatorname{lh}(G)=\lambda=\operatorname{lh}_{\beta}^{\mathcal{U}}$, so $G$ has superstrong type, $\iota_{\alpha}^{\mathcal{T}}=\iota(G)$ and $\delta=\alpha$ but $E_{\alpha}^{\mathcal{T}} \neq G$. Let $\varepsilon=\operatorname{pred}^{\mathcal{U}}(\alpha+1)$. By $4.9, \alpha+1 \in \mathscr{B}^{\mathcal{U}}$ and $\operatorname{cr}(G)=\gamma\left(B_{\varepsilon}^{\mathcal{U}}\right)$. So $\alpha$ is not $\mathcal{U}$ special, so $G$ is a premouse extender. Note $\beta=\alpha+1$ and $F\left(Q_{\alpha+1}^{\mathcal{U}}\right)=E_{\beta}^{\mathcal{U}}=E_{\alpha}^{\mathcal{T}}$. Standard extender factoring arguments (for example, see $[12, \S 5]$ ) now show that there is $\alpha^{\prime}<\alpha$ such that $E_{\alpha^{\prime}}^{\mathcal{T}}=G$. But $\left(\alpha^{\prime}, \alpha\right)<_{\operatorname{lex}}(\alpha, \beta)$, contradiction.

So we may assume that $\lambda=\operatorname{lh}_{\alpha}^{\mathcal{T}}<\operatorname{lh}_{\beta}^{\mathcal{U}}$; so $\alpha<\beta$. Then $\operatorname{ex}_{\beta}^{\mathcal{U}}$ is not a premouse because, letting $\nu=\nu_{\alpha}^{\mathcal{T}}$, we have $\nu<\operatorname{lh}_{\alpha}^{\mathcal{T}}$ and $\operatorname{lh}_{\alpha}^{\mathcal{T}}$ is a cardinal of $\operatorname{ex}_{\beta}^{\mathcal{U}}$, but $E_{\beta}^{\mathcal{U}}\left\lceil\nu \notin \operatorname{ex}_{\beta}^{\mathcal{U}}\right.$. So $\beta$ is $\mathcal{U}$-special. But then $\beta$ is $(\mathcal{U}, \mathcal{T}$ )-unusual (of type (i)). So by induction (with parts 6 and 7 , as $\beta+1<\eta$ ), $\beta$ is not $(\mathcal{T}, \mathcal{U}$ )-unusual. Note then that, by induction, in the rules of comparison, Subcase 4.1 of Case 4 attains at stage $\beta$, so $E_{\beta}^{\mathcal{U}}=\emptyset$, a contradiction.

Part 5: Let $\alpha<\eta$ be $(\mathcal{T}, \mathcal{U})$-unusual. So $S_{\alpha}^{\mathcal{T}}=\{0,1\}$. Let $B^{\mathcal{T}}=B_{\alpha}^{\mathcal{T}}$, $M^{\mathcal{T}}=M_{\alpha}^{\mathcal{T}}$, etc. Let $F=F\left(Q^{\mathcal{T}}\right)$ and $\mu=\operatorname{cr}(F)$.

CAse $1 . \alpha$ is $(\mathcal{T}, \mathcal{U})$-unusual of type (i).

Parts (i), (v)(c): Let us show $\operatorname{lh}_{\xi}^{\mathcal{U}}<\rho^{\mathcal{T}}=\operatorname{lh}(F)$. Suppose not. Then $\operatorname{lh}_{\xi}^{\mathcal{U}}=\rho^{\mathcal{T}}=\operatorname{lh}(F)$ and $E_{\xi}^{\mathcal{U}}=F$. It follows that $E_{\delta}^{\mathcal{T}} \neq \emptyset$ for some $\delta \in[\xi, \alpha)$. Let $\delta$ be least such and $G=E_{\delta}^{\mathcal{T}}$. As in part $4, G$ is a superstrong premouse extender also used in $\mathcal{U}$, contradicting part 4 .

Since $\operatorname{lh}_{\xi}^{\mathcal{U}}<\rho^{\mathcal{T}}, Q^{\mathcal{T}}$ is not a premouse, so $Q$ is type 3 . It easily follows that $\operatorname{lh}_{\xi}^{\mathcal{U}}<\gamma^{\mathcal{T}}$, since if $\gamma$ is a successor cardinal in $Q$ then $Q^{\mathcal{T}}$ is a premouse.

Part (v)(a) ( $Q$ is not superstrong): Suppose otherwise. Then because $Q^{\mathcal{T}}$ is not a premouse, there is $\delta<_{\mathcal{T}} \alpha$ such that $Q_{\delta}^{\mathcal{T}}$ is a premouse and $\operatorname{cr}\left(j_{\delta \alpha}^{\mathcal{T}}\right)=$ $\gamma\left(B_{\delta}^{\mathcal{T}}\right)$ (otherwise $j_{0 \alpha}^{\mathcal{T}}$ is continuous at $\gamma^{B}$ and $Q^{\mathcal{T}}$ is a premouse). So $Q^{\mathcal{T}}$ fails the ISC. So $E_{\xi}^{\mathcal{U}}$ is not a premouse extender and $\xi$ is $\mathcal{U}$-special. But then $F\left(Q_{\xi}^{\mathcal{U}}\right)$ has superstrong type, so $\operatorname{lh}_{\xi}^{\mathcal{U}}=\rho^{\mathcal{T}}$, a contradiction.

$\operatorname{Part}(\mathrm{v})(\mathrm{b})\left(E_{\xi}^{\mathcal{T}}=\emptyset, \alpha=\xi+1\right)$ : We have $i_{F}(\mu)>\rho^{\mathcal{T}}$, so $B_{\xi+1}^{\mathcal{U}} \mid \rho^{\mathcal{T}}=Q^{\mathcal{T}} \| \rho^{\mathcal{T}}$. Now suppose there is $\delta \in[\xi, \alpha)$ such that $E_{\delta}^{\mathcal{T}} \neq \emptyset$. Fix the least such with $\delta+1 \leq_{\mathcal{T}} \alpha$. Let $\varepsilon=\operatorname{pred}^{\mathcal{T}}(\delta+1)$. So $\varepsilon \in \mathscr{B}^{\mathcal{T}}$ and $\kappa=\operatorname{cr}_{\delta}^{\mathcal{T}} \leq \gamma_{\varepsilon}^{\mathcal{T}}$. If $\kappa<\nu\left(F\left(Q_{\varepsilon}^{\mathcal{T}}\right)\right)$ then $\nu(F)>\nu_{\xi}^{\mathcal{U}}$, contradiction. So $\kappa \geq \nu\left(F\left(Q_{\varepsilon}^{\mathcal{T}}\right)\right)$. But then as before, $E_{\delta}^{\mathcal{T}}$ is a premouse extender used in both $\mathcal{T}, \mathcal{U}$, a contradiction. The desired conclusions follow.

Part (v)(d): By an extender factoring as before; otherwise there is $\delta<\xi$ such that $E_{\delta}^{\mathcal{U}}$ is a premouse extender also used in $\mathcal{T}$, a contradiction.

Part (ii) ( $\alpha$ is not $(\mathcal{U}, \mathcal{T})$-unusual): Suppose otherwise. Then $S_{\alpha}^{\mathcal{T}}=\{0,1\}=$ $S_{\alpha}^{\mathcal{U}}$, so $\alpha$ is type (i) with respect to both kinds of unusualness (directly by definition). But by part (v) and symmetry, therefore $\alpha=\xi+1$ with $E_{\xi}^{\mathcal{T}}=\emptyset$ (by $(\mathcal{T}, \mathcal{U})$-unusualness of type (i)), but $E_{\xi}^{\mathcal{T}} \neq \emptyset$ (by $(\mathcal{U}, \mathcal{T})$-unusualness of type (i)), a contradiction.

Part (iii) (Case 3 attains and $B^{\mathcal{T}} \| \rho^{\mathcal{T}}=B^{\mathcal{U}} \mid \rho^{\mathcal{T}}$ ): This is because $\alpha$ is non- $(\mathcal{U}, \mathcal{T})$-unusual, $S_{\alpha}^{\mathcal{T}}=\{0,1\}$ and since by part (v) and its proof, we have $Q^{\mathcal{T}} \| \rho^{\mathcal{T}}=B^{\mathcal{U}} \mid \rho^{\mathcal{T}}$ and $\rho^{\mathcal{T}}<i_{F}(\mu)=i_{\alpha}^{* \mathcal{U}}(\mu)$.

Part (iv): We have $\mu=\operatorname{cr}\left(Q_{\alpha}^{\mathcal{T}}\right)<\gamma_{\alpha}^{\mathcal{T}}$. If $\alpha \in \mathscr{B}^{\mathcal{U}}$ then $\operatorname{cr}\left(F\left(Q_{\alpha}^{\mathcal{U}}\right)\right) \neq \gamma_{\alpha}^{\mathcal{T}}$ since $\alpha=\xi+1$ and $\operatorname{lh}_{\xi}^{\mathcal{U}}<\rho^{\mathcal{T}}<i_{\alpha}^{* \mathcal{U}}(\mu)$. Moreover, $\rho^{\mathcal{T}}$ is a cardinal in $M_{\alpha}^{\mathcal{U}}$, so 
if $\beta \geq \alpha$ and $E_{\beta}^{\mathcal{U}} \neq \emptyset$ then $\rho^{\mathcal{T}} \leq \iota\left(E_{\beta}^{\mathcal{U}}\right)$, which easily gives that if $\beta+1 \in \mathscr{B}^{\mathcal{U}}$ then $\operatorname{cr}\left(F\left(Q_{\beta}^{\mathcal{U}}\right)\right) \neq \gamma_{\alpha}^{\mathcal{T}}$. And at stage $\alpha$, in $\mathcal{T}$ we move into $M^{\mathcal{T}}$, so the rest is similar.

CASE 2. $\alpha$ is $(\mathcal{T}, \mathcal{U})$-unusual of type (ii).

Let $F_{0}=F\left(Q_{\xi_{0}}^{\mathcal{T}}\right)=E_{\xi_{0}}^{\mathcal{U}}, \mu_{0}=\operatorname{cr}\left(F_{0}\right), F_{1}=F\left(Q_{\xi_{1}}^{\mathcal{U}}\right)=E_{\xi_{1}}^{\mathcal{T}}, \mu_{1}=\operatorname{cr}\left(F_{1}\right)$.

Part (ii) ( $\alpha$ is not $(\mathcal{U}, \mathcal{T})$-unusual): By definition of $(\mathcal{T}, \mathcal{U})$-unusual type (ii), $S_{\alpha}^{\mathcal{U}}=\{0\}$

Part (vi)(a) ( $Q$ is superstrong): Suppose not. Let $\delta \in\left[\xi_{0}, \xi_{1}\right)$ be least such that $\delta+1 \leq_{\mathcal{U}} \xi_{1}$. Let $G=E_{\delta}^{\mathcal{U}}$ and $\theta=\operatorname{cr}(G)$. Then $\theta \leq \mu_{1}<i_{G}(\theta)$, so $\mu_{1} \notin \operatorname{rg}\left(j_{0 \xi_{1}}^{\mathcal{U}}\right)$, but $\mu_{1}=\operatorname{cr}\left(F\left(Q_{\xi_{1}}^{\mathcal{U}}\right)\right)$, so $\mu_{1} \in \operatorname{rg}\left(j_{0 \xi_{1}}^{\mathcal{U}}\right)$, contradiction.

$\operatorname{Part}(\mathrm{vi})(\mathrm{c})\left(M_{\alpha}^{* \mathcal{T}}=B_{\xi_{0}}^{\mathcal{T}}\right)$ : Let $\beta<\alpha$ with $E_{\beta}^{\mathcal{T}} \neq \emptyset$, so $\beta \neq \xi_{0}$. If $\beta<\xi_{0}$ then $\iota_{\beta}^{\mathcal{T}} \leq \iota\left(F_{0}\right)$ by $(\dagger)$, and $\iota\left(F_{0}\right)=\mu_{1}$ as $Q$ is superstrong. As $S_{\xi_{0}+1}^{\mathcal{T}}=\{0\}$, $\rho_{\xi_{0}}^{\mathcal{T}} \leq \iota_{\beta}^{\mathcal{T}}$ if $\beta>\xi_{0}$. This suffices.

Parts (i), (iii), (vi)(d): These are easy consequences of the fact that $Q$ is superstong and $\alpha$ type (ii) (in particular, $Q_{\alpha}^{\mathcal{T}}$ fails the ISC, so is not a premouse).

Part (iv): much like in the type (i) case.

Part (vi)(b) (B is exact): We have $\xi_{1} \in \mathscr{B}^{\mathcal{U}}$ and $\operatorname{cr}\left(F\left(Q_{\xi_{1}}^{\mathcal{U}}\right)\right)=\mu_{1}=\iota\left(E_{\xi_{0}}^{\mathcal{U}}\right)$. Let $\varepsilon=\operatorname{pred}^{\mathcal{U}}\left(\xi_{0}+1\right)$. Since $\mu_{1} \in \operatorname{rg}\left(j_{0 \xi_{1}}^{\mathcal{U}}\right)$, it is easy to see that either:

(1) $\xi_{0}+1 \leq_{\mathcal{U}} \xi_{1}\left(\right.$ so $\left.\varepsilon, \xi_{0}+1 \in \mathscr{B}^{\mathcal{U}}\right)$ and $\operatorname{cr}\left(F\left(Q_{\xi_{0}+1}^{\mathcal{U}}\right)\right)=\mu_{1}$ and $\operatorname{cr}\left(F\left(Q_{\varepsilon}^{\mathcal{U}}\right)\right)=$ $\mu_{0}=\operatorname{cr}\left(E_{\xi_{0}}^{\mathcal{U}}\right)$, or

(2) $\xi_{0}+2 \leq_{\mathcal{U}} \xi_{1}\left(\right.$ so $\left.\xi_{0}+2 \in \mathscr{B}^{\mathcal{U}}\right)$ and $\operatorname{cr}\left(F\left(Q_{\xi_{0}+2}^{\mathcal{U}}\right)\right)=\mu_{1}$ and $\varepsilon, \xi_{0}+1 \in \mathscr{B}^{\mathcal{U}}$ and $\gamma\left(B_{\varepsilon}^{\mathcal{U}}\right)=\mu_{0}=\operatorname{cr}\left(E_{\xi_{0}}^{\mathcal{U}}\right)$ and $\gamma\left(B_{\xi_{0}+1}^{\mathcal{U}}\right)=\mu_{1}$ and $E_{\xi_{0}+1}^{\mathcal{U}}=F\left(Q_{\xi_{0}+1}^{\mathcal{U}}\right)$.

We claim that (1) holds, so suppose (2) holds. Now $E_{\varepsilon}^{\mathcal{U}} \neq \emptyset$ just by definition, as $\varepsilon=\operatorname{pred}^{\mathcal{U}}\left(\xi_{0}+1\right)$. We have $M_{\xi_{0}+1}^{* \mathcal{U}}=B_{\varepsilon}^{\mathcal{U}}$ and by the normality rules, $\operatorname{cr}\left(E_{\xi_{0}}^{\mathcal{U}}\right)=\gamma\left(B_{\varepsilon}^{\mathcal{U}}\right)<\iota\left(E_{\varepsilon}^{\mathcal{U}}\right)$, and since $Q$ is superstrong, therefore $E_{\varepsilon}^{\mathcal{U}} \notin \mathbb{E}_{+}\left(Q_{\varepsilon}^{\mathcal{U}}\right)$. So by part $3 \mathrm{~d}, S_{\varepsilon}^{\mathcal{T}}=\{0\}$, so there is $\varepsilon^{\prime}<\varepsilon$ with $B_{\varepsilon^{\prime}}^{\mathcal{U}}=B_{\varepsilon}^{\mathcal{U}}$, where at stage $\varepsilon^{\prime}$ we move into $M_{\varepsilon^{\prime}}^{\mathcal{U}}=M_{\varepsilon}^{\mathcal{U}}$ in $\mathcal{U}$. Note that $\varepsilon^{\prime}$ is not $(\mathcal{U}, \mathcal{T})$-unusual, by part (iv) and since $\gamma\left(B_{\varepsilon}^{\mathcal{U}}\right)=\mu_{0}=\operatorname{cr}\left(F\left(Q_{\xi_{0}}^{\mathcal{T}}\right)\right.$ ). And $\varepsilon^{\prime}$ is not $(\mathcal{T}, \mathcal{U})$-unusual (type (ii)) as $S_{\varepsilon^{\prime}}^{\mathcal{U}}=\{0,1\}$. So by $3 \mathrm{a}$ and $3 \mathrm{c}, E_{\varepsilon^{\prime}}^{\mathcal{T}}=F\left(Q_{\varepsilon}^{\mathcal{U}}\right)$ and $E_{\varepsilon^{\prime}}^{\mathcal{U}}=\emptyset$. But then $\xi_{0}+1$ is $(\mathcal{U}, \mathcal{T})$-unusual, so $E_{\xi_{0}+1}^{\mathcal{U}}=\emptyset \neq F\left(Q_{\xi_{0}+1}^{\mathcal{U}}\right)$, contradiction.

Since $E_{\xi_{0}}^{\mathcal{U}}$ is total over $B_{\varepsilon}^{\mathcal{U}},\left(\mu_{0}{ }^{++}\right)^{Q_{\xi_{0}}^{\mathcal{T}}} \leq\left(\mu_{0}{ }^{++}\right)^{B_{\varepsilon}^{\mathcal{U}}}$ and $\left(Q_{\xi_{0}}^{\mathcal{T}} \sim B_{\varepsilon}^{\mathcal{U}}\right) \|\left(\mu_{0}{ }^{++}\right)^{Q_{\xi_{0}}^{\mathcal{T}}}$. Let $U_{0}=\operatorname{Ult}\left(Q_{\xi_{0}}^{\mathcal{T}}, F_{0}\right)$. Since $k=i_{F_{0}}^{Q_{\xi_{0}}^{\mathcal{T}}}$ is continuous at $\left(\mu_{0}{ }^{++}\right)^{Q_{\xi_{0}}^{\mathcal{T}}}$, then $\left(\mu_{1}^{++}\right)^{U_{0}} \leq\left(\mu_{1}^{++}\right)^{B_{\xi_{0}+1}^{\tau}}$ and $\left(U_{0} \sim B_{\xi_{0}+1}^{\mathcal{U}}\right) \|\left(\mu_{1}^{++}\right)^{U_{0}}$.

Suppose $B$ is not exact. By 3.26 , neither is $B_{\xi_{0}}^{\mathcal{T}}$. So $\left(\mu_{1}^{++}\right)^{M_{\xi_{0}}^{\mathcal{T}}}<\left(\mu_{1}^{++}\right)^{U_{0}}$ and $\left(M_{\xi_{0}}^{\mathcal{T}} \sim U_{0} \sim B_{\xi_{0}+1}^{\mathcal{U}}\right) \|\left(\mu_{1}^{++}\right)^{M_{\xi_{0}}^{\mathcal{T}}}$, but by non-triviality, $M_{\xi_{0}}^{\mathcal{T}} \Varangle U_{0}$. So $M_{\xi_{0}}^{\mathcal{T}} \nrightarrow B_{\xi_{0}+1}^{\mathcal{U}}$. We have $E_{\xi_{0}}^{\mathcal{T}}=\emptyset$ and $S_{\xi_{0}+1}^{\mathcal{T}}=\{0\}$ and $M_{\xi_{0}+1}^{\mathcal{T}}=M_{\xi_{0}}^{\mathcal{T}}$. So

$$
\left(\mu_{1}{ }^{++}\right)^{M_{\xi_{0}}^{\mathcal{T}}} \geq\left(\mu_{1}^{++}\right)^{\operatorname{ex}_{\xi_{1}}^{\mathcal{T}}}=\left(\mu_{1}{ }^{++}\right)^{B_{\xi_{1}}^{\mathcal{U}}}=\left(\mu_{1}{ }^{++}\right)^{B_{\xi_{0}+1}^{\mathcal{U}}}>\left(\mu_{1}{ }^{++}\right)^{M_{\xi_{0}}^{\mathcal{T}}},
$$

contradiction. 
Part (vi)(e): Arguing as just above, we have $\mu_{0}^{\prime \prime}=\left(\mu_{0}^{++}\right)^{B_{\varepsilon}^{\mathcal{U}}}=\left(\mu_{0}{ }^{++}\right)^{Q_{\xi_{0}}^{\mathcal{T}}}$ and $\left(Q_{\xi_{0}}^{\mathcal{T}} \sim \operatorname{ex}_{\xi_{0}}^{\mathcal{U}} \sim B_{\varepsilon}^{\mathcal{U}}\right) \| \mu_{0}^{\prime \prime}$. By exactness on both sides, this easily gives the claimed agreement between $M_{\alpha}^{\mathcal{T}}$ and $M_{\alpha}^{\mathcal{U}}$.

Part 6 is symmetric.

Part 3b: Suppose $\alpha+1<\eta$ and $\alpha$ is $(\mathcal{T}, \mathcal{U})$-unusual. By part 5, Case 3 attains, $\alpha$ is non- $(\mathcal{U}, \mathcal{T})$-unusual, and $M_{\alpha}^{\mathcal{U}} \mid \rho_{\alpha}^{\mathcal{T}}$ is passive, so Subcase 3.1 attains and $E_{\alpha}^{\mathcal{T}}=\emptyset=E_{\alpha}^{\mathcal{U}}$.

Part 7 follows similarly from part 5 .

Claim 2. Suppose $Q$ is type $3, S_{\xi_{1}}^{\mathcal{U}}=\{0,1\}, E_{\xi_{1}}^{\mathcal{T}}=F\left(Q_{\xi_{1}}^{\mathcal{U}}\right), \xi_{1}+1 \in \mathscr{B}^{\mathcal{T}}$ and $\operatorname{cr}_{\xi_{1}}^{\mathcal{T}}=\gamma_{\varepsilon}^{\mathcal{T}}$ where $\varepsilon=\operatorname{pred}^{\mathcal{T}}\left(\xi_{1}+1\right)$. Then $\xi_{1}+1$ is $(\mathcal{T}, \mathcal{U})$-unusual of type (ii).

Proof. Suppose not. Note that $E_{\varepsilon}^{\mathcal{T}} \in \mathbb{E}_{+}\left(M_{\varepsilon}^{\mathcal{T}}\right) \backslash \mathbb{E}_{+}\left(Q_{\varepsilon}^{\mathcal{T}}\right)$, so $\xi_{0}=\operatorname{movin}^{\mathcal{T}}(\varepsilon)$ exists. If $E_{\xi_{0}}^{\mathcal{U}}=F\left(Q_{\xi_{0}}^{\mathcal{T}}\right)$ then $\xi_{1}+1$ is $(\mathcal{T}, \mathcal{U})$-unusual type (ii). But if $E_{\xi_{0}}^{\mathcal{U}}=\emptyset$ then, as $E_{\xi_{0}}^{\mathcal{T}}=\emptyset, \xi_{0}$ is $(\mathcal{T}, \mathcal{U})$-unusual, so by Claim 1(5) (iv) $\gamma_{\xi_{0}}^{\mathcal{T}} \neq \operatorname{cr}\left(F\left(Q_{\xi_{1}}^{\mathcal{U}}\right)\right)$, contradiction.

Claim 3. The comparison terminates at some countable stage.

Proof. We may assume that $Q$ is type 3 , since otherwise every extender used in $(\mathcal{T}, \mathcal{U})$ is a premouse extender and the usual argument works.

Suppose $(\mathcal{T}, \mathcal{U})$ reaches length $\omega_{1}+1$. Let $\eta \in$ OR be large, $\varrho: X \rightarrow V_{\eta}$ be elementary with $X$ countable transitive and everything relevant in $\operatorname{rg}(\varrho)$. Let $\mu=\operatorname{cr}(\varrho)$. Let $W=B_{\omega_{1}}^{\mathcal{T}}\left\|\omega_{1}=B_{\omega_{1}}^{\mathcal{U}}\right\| \omega_{1}$. As is routine, either $i_{\mu \omega_{1}}^{\mathcal{T}}$ or $j_{\mu \omega_{1}}^{\mathcal{T}}$ is defined, if $i_{\mu \omega_{1}}^{\mathcal{T}}$ is defined then $\left(\mu^{+}\right)^{M_{\mu}^{\mathcal{T}}}=\left(\mu^{+}\right)^{W}$ and $\left(M_{\mu}^{\mathcal{T}} \sim W\right) \|\left(\mu^{+}\right)^{W}$ and $i_{\mu \omega_{1}}^{\mathcal{T}} \subseteq \varrho$ and likewise if $j_{\mu, \omega_{1}}^{\mathcal{T}}$ is defined. Likewise for $\mathcal{U}$.

Let $\xi_{0}$ be least such that $E_{\xi_{0}}^{\mathcal{T}} \neq \emptyset$ and $\kappa<\mathcal{T} \xi_{0}+1<\mathcal{T} \omega_{1}$, and $\alpha$ likewise for $\mathcal{U}$. Let us assume that $\xi_{0} \leq \alpha$; otherwise it is symmetric. Let $\iota=\iota_{\xi_{0}}^{\mathcal{T}} \leq \iota_{\alpha}^{\mathcal{T}}$. As usual, $E_{\xi_{0}}^{\mathcal{T}} \uparrow \iota=E_{\alpha}^{\mathcal{U}} \uparrow \iota$.

SubClaim 3.1. We have:

(a) The trivial completion of $E_{\xi_{0}}^{\mathcal{T}}\left\lceil\nu_{\xi_{0}}^{\mathcal{T}}\right.$ is a premouse extender.

(b) $\xi_{0}<\alpha$ and $\iota_{\xi_{0}}^{\mathcal{T}}<\iota_{\alpha}^{\mathcal{U}}$ and $\nu_{\xi_{0}}^{\mathcal{T}}<\nu_{\alpha}^{\mathcal{U}}$ and $\operatorname{lh}_{\xi_{0}}^{\mathcal{T}}<\operatorname{lh}_{\alpha}^{\mathcal{U}}$.

(c) $E_{\alpha}^{\mathcal{U}}\left\lceil\nu_{\xi_{0}}^{\mathcal{T}} \notin \mathrm{ex}_{\alpha}^{\mathcal{U}}\right.$, so ex $\mathcal{U}_{\alpha}^{\mathcal{U}}$ is not a premouse and $\alpha$ is $\mathcal{U}$-special.

Proof. Part (a): We have $\nu_{\xi_{0}}^{\mathcal{T}} \leq \nu_{\alpha}^{\mathcal{U}}$ because $\iota_{\xi_{0}}^{\mathcal{T}} \leq \iota_{\alpha}^{\mathcal{U}}$ and by compatibility. So part (a) follows from standard extender factoring (otherwise we get some premouse extender which factors into $E_{\xi_{0}}^{\mathcal{T}}$, used in both $\mathcal{T}, \mathcal{U}$; cf. [12, §5]).

Part (b): If $\iota_{\xi_{0}}^{\mathcal{T}}=\iota_{\alpha}^{\mathcal{U}}$ then $E_{\xi_{0}}^{\mathcal{T}}=E_{\alpha}^{\mathcal{U}}$, contradicting Claim 1(4). So $\iota_{\xi_{0}}^{\mathcal{T}}<\iota_{\alpha}^{\mathcal{U}}$, so $\xi_{0}<\alpha$. We have $\nu_{\xi_{0}}^{\mathcal{T}} \leq \nu_{\alpha}^{\mathcal{U}}$. But $\nu_{\xi_{0}}^{\mathcal{T}} \neq \nu_{\alpha}^{\mathcal{U}}$ by Claim 1(4). So $\nu_{\xi_{0}}^{\mathcal{T}}<\nu_{\alpha}^{\mathcal{U}}$. We have $\operatorname{lh}_{\xi_{0}}^{\mathcal{T}} \leq \operatorname{lh}_{\alpha}^{\mathcal{U}}$. Suppose $\operatorname{lh}_{\xi_{0}}^{\mathcal{T}}=\lambda=\operatorname{lh}_{\alpha}^{\mathcal{U}}$. Let $P=\operatorname{ex}_{\alpha}^{\mathcal{U}}$ and $\delta=\operatorname{lgcd}(P)=$ $\operatorname{lgcd}\left(\operatorname{ex}_{\xi_{0}}^{\mathcal{T}}\right)$. Then $E_{\xi_{0}}^{\mathcal{T}} \notin P$. Since $\nu_{\xi_{0}}^{\mathcal{T}}<\nu_{\alpha}^{\mathcal{U}}$ and by part (a), therefore $P$ is not a 
premouse. So $\alpha$ is $\mathcal{U}$-special, so $\iota_{\xi_{0}}^{\mathcal{T}}<\delta=\iota_{\alpha}^{\mathcal{U}}$. But $\iota_{\xi_{0}}^{\mathcal{T}} \geq \delta$ as $\delta=\operatorname{lgcd}\left(\operatorname{ex}_{\xi_{0}}^{\mathcal{T}}\right)$, a contradiction. So $\mathrm{lh}_{\xi_{0}}^{\mathcal{T}}<\mathrm{lh}_{\alpha}^{\mathcal{U}}$.

Part (c): $\operatorname{lh}_{\xi_{0}}^{\mathcal{T}}$ is a cardinal of $P=\mathrm{ex}_{\alpha}^{\mathcal{U}}$ and $E_{\xi_{0}}^{\mathcal{T}} \notin P$, by 4.9 and agreement between models of $\mathcal{T}$ and $\mathcal{U}$. Since $\nu_{\xi_{0}}^{\mathcal{T}}<\nu_{\alpha}^{\mathcal{T}}$ and by part (a), $P$ fails the ISC, and so $\alpha$ is $\mathcal{U}$-special.

Let $\varepsilon<\mathcal{U} \alpha$ be largest such that $F\left(Q_{\varepsilon}^{\mathcal{U}}\right)\left\lceil\nu\left(F\left(Q_{\varepsilon}^{\mathcal{U}}\right)\right)\right.$ satisfies the ISC. Let $\xi_{1}+1=\min \left((\varepsilon, \alpha]_{\mathcal{U}}\right)$. So $E_{\xi_{1}}^{\mathcal{U}} \neq \emptyset$ but $\xi_{1}$ is not $\mathcal{U}$-special. Let $F_{0}=F\left(Q_{\varepsilon}^{\mathcal{U}}\right)$; then $E_{\xi_{0}}^{\mathcal{T}}\left\lceil\nu_{\xi_{0}}^{\mathcal{T}}=F_{0}\left\lceil\nu\left(F_{0}\right)\right.\right.$. Let $\delta$ be least such that $E_{\delta}^{\mathcal{T}} \neq \emptyset$ and $\xi_{0}+1<\mathcal{T} \delta+1<\mathcal{T} \omega_{1}$. Let $\iota_{1}=\min \left(\iota_{\xi_{1}}^{\mathcal{U}}, \iota_{\delta}^{\mathcal{T}}\right)$. Extender factoring gives $E_{\delta}^{\mathcal{T}}\left\lceil\iota_{1}=E_{\xi_{1}}^{\mathcal{U}}\left\lceil\iota_{1}\right.\right.$.

SubClaim 3.2. (a) $\operatorname{ex}_{\xi_{1}}^{\mathcal{U}}$ is a premouse, (b) $\delta>\xi_{1}$ and $\iota_{\delta}^{\mathcal{T}}>\iota_{\xi_{1}}^{\mathcal{U}}$ and $\nu_{\delta}^{\mathcal{T}}>\nu_{\xi_{1}}^{\mathcal{U}}$ and $\operatorname{lh}_{\delta}^{\mathcal{T}}>\operatorname{lh}_{\xi_{1}}^{\mathcal{U}}$, (c) $E_{\delta}^{\mathcal{T}}\left\lceil\nu_{\xi_{1}}^{\mathcal{U}} \notin \mathrm{ex}_{\delta}^{\mathcal{T}}\right.$, so ex $\operatorname{ex}_{\delta}^{\mathcal{T}}$ fails the ISC and $\delta$ is $\mathcal{T}$-special.

Proof. Like Subclaim 3.1 and because $\xi_{1}$ is not $\mathcal{U}$-special.

SubClaim 3.3. $Q_{\alpha}^{\mathcal{U}}=Q_{\xi_{1}+1}^{\mathcal{U}}$, so $E_{\alpha}^{\mathcal{U}}$ is equivalent to $E_{\xi_{1}}^{\mathcal{U}} \circ E_{\xi_{0}}^{\mathcal{T}}$.

Proof. Suppose not. Fix $\gamma^{\prime}$ least with $E_{\gamma^{\prime}}^{\mathcal{U}} \neq \emptyset$ and $\xi_{1}+1<_{\mathcal{U}} \gamma^{\prime}+1 \leq \mathcal{U} \alpha$. Fix $\gamma$ least with $E_{\gamma}^{\mathcal{T}} \neq \emptyset$ and $\gamma+1<_{\mathcal{T}} \delta$ and $F\left(Q_{\gamma+1}^{\mathcal{T}}\right)\left\lceil\nu\left(F\left(Q_{\gamma+1}^{\mathcal{T}}\right)\right)\right.$ fails the ISC. Then both $\operatorname{ex}_{\gamma}^{\mathcal{T}}$ and $\operatorname{ex}_{\gamma^{\prime}}^{\mathcal{U}}$ are premice, and extender factoring gives $\operatorname{ex}_{\gamma}^{\mathcal{T}}=\operatorname{ex}_{\gamma^{\prime}}^{\mathcal{T}}$, contradiction.

SubClaim 3.4. $Q$ is superstrong and $\xi_{1}+1$ is $(\mathcal{U}, \mathcal{T})$-unusual type (ii).

Proof. Let $\mu_{0}=\mu=\operatorname{cr}\left(E_{\xi_{0}}^{\mathcal{T}}\right)=\operatorname{cr}\left(Q_{\varepsilon}^{\mathcal{U}}\right)$. Recall $F_{0}=F\left(Q_{\varepsilon}^{\mathcal{U}}\right)$. Let $\mu_{1}=$ $\operatorname{cr}\left(E_{\delta}^{\mathcal{T}}\right)=\operatorname{cr}\left(E_{\xi_{1}}^{\mathcal{U}}\right)$. As $M_{\xi_{1}+1}^{* \mathcal{U}}=B_{\varepsilon}^{\mathcal{U}}$, we have $\mu_{1} \leq \gamma_{\varepsilon}^{\mathcal{U}} \leq i_{F_{0}}\left(\mu_{0}\right)$. And as $\delta$ is $\mathcal{T}$-special, we have $\xi_{0}+1=\operatorname{pred}^{\mathcal{T}}(\delta+1) \leq \mathcal{T} \delta$ and

$$
\mu_{1}=\operatorname{cr}\left(F\left(Q_{\delta}^{\mathcal{T}}\right)\right)=i_{0 \delta}^{\mathcal{T}}\left(\operatorname{cr}\left(F^{Q}\right)\right)=i_{0, \xi_{0}+1}^{\mathcal{T}}\left(\operatorname{cr}\left(F^{Q}\right)\right),
$$

so $\mu_{1} \in \operatorname{rg}\left(i_{\mu_{0}, \xi_{0}+1}^{\mathcal{T}}\right)$. But $\mu_{1}>\mu_{0}$, so $\mu_{1} \geq i_{\mu_{0}, \xi_{0}+1}^{\mathcal{T}}\left(\mu_{0}\right)$. But $E_{\xi_{0}}^{\mathcal{T}}$ is equivalent to $F_{0}$, so putting things together, $i_{\mu_{0}, \xi_{0}+1}^{\mathcal{T}}\left(\mu_{0}\right)=\mu_{1}=\gamma_{\varepsilon}^{\mathcal{U}}=i_{F_{0}}\left(\mu_{0}\right)$.

It follows that $Q_{\varepsilon}^{\mathcal{U}}$ and $Q$ are superstrong, $Q_{\varepsilon}^{\mathcal{U}}$ is therefore a premouse, $\nu\left(F_{0}\right)=\mu_{1}=\nu\left(E_{\xi_{0}}^{\mathcal{T}}\right)$ and $\operatorname{ex}_{\xi_{0}}^{\mathcal{T}}=Q_{\varepsilon}^{\mathcal{U}}=Q_{\xi_{0}}^{\mathcal{U}}$ (and $B_{\varepsilon}^{\mathcal{U}}=B_{\xi_{0}}^{\mathcal{U}}$, though maybe $\left.\varepsilon>\xi_{0}\right)$. Therefore in $\mathcal{U}$, we move into $M_{\xi_{0}}^{\mathcal{U}}$ at stage $\xi_{0}$, and $E_{\xi_{0}}^{\mathcal{T}}=F\left(Q_{\xi_{0}}^{\mathcal{U}}\right)$, and $\gamma_{\xi_{0}}^{\mathcal{U}}=\mu_{1}$.

Let $\varphi<\mathcal{T} \delta$ be largest such that $F\left(Q_{\varphi}^{\mathcal{T}}\right)\left\lceil\nu\left(Q_{\varphi}^{\mathcal{T}}\right)\right.$ satisfies the ISC. Since $Q$ is superstrong, $Q_{\varphi}^{\mathcal{T}}$ is a superstrong premouse, so $\nu\left(Q_{\varphi}^{\mathcal{T}}\right)=\gamma_{\varphi}^{\mathcal{T}}$. By the claims above, $\gamma_{\varphi}^{\mathcal{T}}=\nu\left(E_{\xi_{1}}^{\mathcal{U}}\right)$ and in fact $Q_{\varphi}^{\mathcal{T}}=\operatorname{ex}_{\xi_{1}}^{\mathcal{U}}$, and hence $Q_{\xi_{1}}^{\mathcal{T}}=\operatorname{ex}_{\xi_{1}}^{\mathcal{U}}$ (and $B_{\varphi}^{\mathcal{T}}=B_{\xi_{1}}^{\mathcal{T}}$, though maybe $\varphi>\xi_{1}$ ), and in $\mathcal{T}$, we move into $M_{\xi_{1}}^{\mathcal{T}}$ at stage $\xi_{1}$.

We have therefore established that $\xi_{1}+1$ is $(\mathcal{U}, \mathcal{T})$-unusual of type (ii), as witnessed by $\xi_{0}, \xi_{1}$. 
By the last subclaim and rules of comparison, $E_{\xi_{1}+1}^{\mathcal{T}}=\emptyset=E_{\xi_{1}+1}^{\mathcal{U}}$, and in $\mathcal{U}$, we move into $M_{\xi_{1}+1}^{\mathcal{U}}$ at stage $\xi_{1}+1$. So $\xi_{1}+2 \in \mathscr{M}^{\mathcal{U}}$ and $M_{\xi_{1}+2}^{\mathcal{U}}=M_{\xi_{1}+1}^{\mathcal{U}}$, so note $\operatorname{OR}\left(Q_{\xi_{1}+1}^{\mathcal{U}}\right)<\zeta=\operatorname{lh}\left(E_{\xi_{1}+2}^{\mathcal{T}}\right)$ or $\operatorname{OR}\left(Q_{\xi_{1}+1}^{\mathcal{U}}\right)<\zeta=\operatorname{lh}\left(E_{\xi_{1}+2}^{\mathcal{U}}\right)$, whichever extender is defined. But $E_{\alpha}^{\mathcal{U}}=F\left(Q_{\alpha}^{\mathcal{U}}\right)$ and $\alpha \geq \xi_{1}+1$, so $\alpha>\xi_{1}+1$, but by Subclaim 3.3, $Q_{\alpha}^{\mathcal{U}}=Q_{\xi_{1}+1}^{\mathcal{U}}$, $\operatorname{so} \ln \left(E_{\alpha}^{\mathcal{U}}\right)<\zeta$, a contradiction, completing the proof that comparison terminates.

We now analyse the manner in which the comparison terminates. Let $\alpha+1=$ $\operatorname{lh}(\mathcal{T}, \mathcal{U})$. Let $B^{\mathcal{T}}=B_{\alpha}^{\mathcal{T}}$, etc. We say that the comparison terminates early if $\alpha=\beta+1$ for some $\beta$ and $E_{\beta}^{\mathcal{T}}=\emptyset=E_{\beta}^{\mathcal{U}}$. We begin with the non-exceptional case:

Claim 4. Suppose that $B$ is non-exceptional. Then:

- $\alpha \in \mathscr{B}^{\mathcal{T}} \Delta \mathscr{B}^{\mathcal{U}}$ and $\operatorname{card}\left(S^{\mathcal{T}}\right)=\operatorname{card}\left(S^{\mathcal{U}}\right)=1$ and $\mathfrak{M}^{\mathcal{T}}=\mathfrak{M}^{\mathcal{U}}$.

- $m \geq 0$ and the cephalanx $C \in\left\{B^{\mathcal{T}}, B^{\mathcal{U}}\right\}$ has a good core.

Proof. Note that $(*)$ either $B$ is non-exact or $Q$ is non-superstrong, because $B$ is non-exceptional and by line $(6)$. So if any $\beta$ is $(\mathcal{T}, \mathcal{U})$ - or $(\mathcal{U}, \mathcal{T})$-unusual, by Claim 1, it is type (i).

Suppose that $\operatorname{card}\left(S^{\mathcal{U}}\right)=2$, so $\mathfrak{M}^{\mathcal{T}}=\{Z\}$ with $Z \triangleleft B^{\mathcal{U}}$. So $Z$ is sound, $\alpha \in \mathscr{B}^{\mathcal{T}}, \beta=\operatorname{movin}^{\mathcal{T}}(\alpha)$ is defined and $M^{\mathcal{T}}=Z=M_{\beta}^{\mathcal{T}}$. So either (a) $E_{\beta}^{\mathcal{U}}=F\left(Q_{\beta}^{\mathcal{T}}\right)$ or $(\mathrm{b})\left[\beta=\xi+1\right.$ is $(\mathcal{T}, \mathcal{U})$-unusual type (i) and $E_{\xi}^{\mathcal{U}}$ is equivalent to $\left.F\left(Q_{\beta}^{\mathcal{T}}\right)\right]$. If $B, B_{\beta}^{\mathcal{T}}$ are non-exact then since they are non-trivial, $M_{\beta}^{\mathcal{T}} \neq$ $N^{B_{\beta}^{\mathcal{T}}} \triangleleft B^{\mathcal{U}}$, contradicting that $M_{\beta}^{\mathcal{T}}=M^{\mathcal{T}} \triangleleft B^{\mathcal{U}}$. If $B, B_{\beta}^{\mathcal{T}}$ are exact, so $Q, Q_{\beta}^{\mathcal{T}}$ non-superstrong, then note that we get enough agreement that $\left(\left(\rho_{\beta}^{\mathcal{T}}\right)^{+}\right)^{M_{\beta}^{\mathcal{T}}}=$ $\left(\left(\rho_{\beta}^{\mathcal{T}}\right)^{+}\right)^{B_{\beta}^{\mathcal{U}+1}}$, which again gives a contradiction. Likewise $\operatorname{card}\left(S^{\mathcal{T}}\right)=1$.

So $\operatorname{card}\left(S^{\mathcal{T}}\right)=\operatorname{card}\left(S^{\mathcal{U}}\right)=1$. Likewise $\mathfrak{M}^{\mathcal{T}}=\mathfrak{M}^{\mathcal{U}}$. We have $\alpha \in \mathscr{B}^{\mathcal{T}} \cup \mathscr{B}^{\mathcal{U}}$ as usual. Suppose $\alpha \in \mathscr{B}^{\mathcal{T}} \cap \mathscr{B}^{\mathcal{U}}$. Let $\beta^{\mathcal{T}}=\operatorname{movin}^{\mathcal{T}}(\alpha)$ and $\beta^{\mathcal{U}}=\operatorname{movin}^{\mathcal{U}}(\alpha)$. Then $\beta^{\mathcal{T}} \neq \beta^{\mathcal{U}}$, so suppose $\beta^{\mathcal{T}}<\beta^{\mathcal{U}}$. Then $E_{\gamma}^{\mathcal{T}}=\emptyset$ for all $\gamma \geq \beta^{\mathcal{T}}$, and hence $\beta^{\mathcal{U}}$ is $(\mathcal{U}, \mathcal{T})$-unusual, hence type (i) (see above), so $\beta^{\mathcal{U}}=\xi+1$ and $E_{\xi}^{\mathcal{T}} \neq \emptyset$, contradiction.

So $\alpha \in \mathscr{B}^{\mathcal{T}} \Delta \mathscr{B}^{\mathcal{U}}$ and we may assume $\alpha \in \mathscr{B}^{\mathcal{T}} \backslash \mathscr{B}^{\mathcal{U}}$. So $S^{\mathcal{T}}=\{0\}$ and letting $\mathfrak{M}^{\mathcal{T}}=\{Z\}=\mathfrak{M}^{\mathcal{U}}, Z=M^{\mathcal{T}}=B^{\mathcal{U}}$ is unsound. We need to show that $m \geq 0$ and $B^{\mathcal{T}}$ has a good core. Let $\beta=\operatorname{movin}^{\mathcal{T}}(\alpha)$. Basically as above, $E_{\gamma}^{\mathcal{T}}=\emptyset \neq E_{\gamma}^{\mathcal{U}}$ for all $\gamma>\beta$.

CASE I. $\beta$ is $(\mathcal{T}, \mathcal{U})$-unsual (equivalently, $E_{\beta}^{\mathcal{T}}=\emptyset=E_{\beta}^{\mathcal{U}}$ ).

So $\beta=\xi+1$ is type (i), $E_{\xi}^{\mathcal{U}}$ is equivalent to $F\left(Q^{\mathcal{T}}\right)$, and $Q$ is type 3 but not superstrong. We have $B_{\beta}^{\mathcal{U}}=B_{\beta+1}^{\mathcal{U}}$ and $S_{\beta}^{\mathcal{U}}=S_{\beta+1}^{\mathcal{U}}$ and $\left(M^{\mathcal{T}} \sim B_{\beta+1}^{\mathcal{U}}\right) \mid \rho^{\mathcal{T}}$.

SuBCASE I.1. $M^{\mathcal{T}} \neq B_{\beta+1}^{\mathcal{U}}$.

Then $E_{\beta+1}^{\mathcal{U}} \neq \emptyset$ and $\rho^{\mathcal{T}}<\operatorname{lh}_{\beta+1}^{\mathcal{U}}$ and $\rho^{\mathcal{T}}$ is a successor cardinal of $B_{\beta+1}^{\mathcal{U}}$. So $\rho^{\mathcal{T}} \leq \iota_{\beta+1}^{\mathcal{U}}$. Since $M^{\mathcal{T}}$ is $\rho^{\mathcal{T}}$-sound, it follows that there is exactly one ordinal $\delta$ such that $\delta \geq \beta+1$ and $\delta+1 \leq_{\mathcal{U}} \alpha$, and in fact $\delta+1=\alpha$. So ex $\mathcal{U}_{\delta}^{\mathcal{U}}$ is a premouse, as $\alpha \notin \mathscr{B}^{\mathcal{U}}$. Since $M^{\mathcal{T}}$ is $\rho^{\mathcal{T}}$-sound, therefore $\delta=\beta+1$ and $E_{\beta+1}^{\mathcal{U}}$ is type 1 or 
type 3, with $\operatorname{lh}_{\beta+1}^{\mathcal{U}}=\left(\left(\rho^{\mathcal{T}}\right)^{+}\right)^{M^{\mathcal{T}}}$. It follows that $m \geq 0$ and $B^{\mathcal{T}}$ is non-exact, and letting $F^{*}=F\left(N^{B^{\mathcal{T}}}\right)$ and $\kappa^{*}=\operatorname{cr}\left(F^{*}\right)$, we have $E_{\beta+1}^{\mathcal{U}}=F^{*}$, and $M^{\mathcal{T}}$ has an $\left(m, \rho^{\mathcal{T}}\right)$-good core at $\kappa^{*}$, and $G_{m, \kappa^{*}, \rho^{\mathcal{T}}}^{M^{\mathcal{T}}}=F^{*} \uparrow \rho^{\mathcal{T}}$ and $H_{m, \kappa^{*}}^{M^{\mathcal{T}}}=B_{\alpha}^{* \mathcal{U}}$. Also, if $F^{*}$ is type 1 then $\operatorname{pred}^{\mathcal{U}}(\alpha)=\beta+1$ and $\beta+1 \notin \mathscr{B}^{\mathcal{U}}$ and $B_{\alpha}^{* \mathcal{U}}=B_{\beta+1}^{\mathcal{U}}$, and $M^{\mathcal{T}}$ has an $\left(m, \gamma^{\mathcal{T}}\right)$-good core at $\operatorname{cr}\left(F\left(Q^{\mathcal{T}}\right)\right)$, etc. So $B^{\mathcal{T}}$ has a good core.

SubCASE I.2. $M^{\mathcal{T}}=Z=B_{\beta+1}^{\mathcal{U}}$.

This is a simplification of the previous case, but here, the comparison terminates early (so $\alpha=\beta+1$ ), and $B^{\mathcal{T}}, B$ are exact.

CASE II. $\beta$ is not $(\mathcal{T}, \mathcal{U})$-unusual (so $E_{\beta}^{\mathcal{U}}=F^{\prime}$ where $F^{\prime}=F\left(Q^{\mathcal{T}}\right)$ ).

SubCASE II.1. $Q$ is not superstrong.

So $F^{\prime}$ does not have superstrong type. Things work much as in the previous case, but there are a couple more possibilities, which we just outline. If $B$ is exact then $\alpha=\beta+1$, and $F^{\prime}$ is the last extender used in $\mathcal{U}$. If $B$ is non-exact then $\alpha=\beta+2$ and like above, $F^{*}=F\left(N^{B^{\mathcal{T}}}\right)$ is type 1 or type 3 and is the last extender used in $\mathcal{U}$. Here if $B$ is non-exact with $N^{B^{\mathcal{T}}}$ is type 1 and $Q^{\mathcal{T}}$ type 2 then $Q^{\mathcal{T}}=B_{\alpha}^{* \mathcal{U}}$.

SubCase II.2. $Q$ is superstrong.

So $F^{\prime}$ has superstrong type, so by $(*)$ above, $B$ is non-exact. Things work much as before, but there are some extra details, which we just illustrate in an example case. Let $\varepsilon=\operatorname{pred}^{\mathcal{U}}(\beta+1)$. Note first that if $\beta+1 \in \mathscr{B}^{\mathcal{U}}$ then $\rho^{\mathcal{T}}<$ $\rho_{\beta+1}^{\mathcal{U}}$, by Claim 2 and non-exactness. Now $\kappa^{\prime}=\operatorname{cr}\left(F^{\prime}\right)<\iota_{\varepsilon}^{\mathcal{U}}$ and $\left(\left(\kappa^{\prime}\right)^{+}\right)^{Q^{\mathcal{T}}} \leq$ $\operatorname{lh}_{\varepsilon}^{\mathcal{U}}$. Suppose for example that $\left(\left(\kappa^{\prime}\right)^{+}\right)^{Q^{\mathcal{T}}}=\operatorname{lh}_{\varepsilon}^{\mathcal{U}}$. Then $E_{\varepsilon}^{\mathcal{U}}$ is type 2 and $B_{\beta+1}^{* \mathcal{U}}=$ $\operatorname{ex}_{\varepsilon}^{\mathcal{U}}$, and $\operatorname{OR}\left(B_{\beta+1}^{\mathcal{U}}\right)=\operatorname{OR}\left(Q^{\mathcal{T}}\right)$ and $B_{\beta+1}^{\mathcal{U}}$ is active type 2 , so $E_{\beta+1}^{\mathcal{U}}=F\left(B_{\beta+1}^{\mathcal{U}}\right)$. Note that $\left(Q^{\mathcal{T}} \sim B_{\varepsilon+1}^{\mathcal{U}}\right) \|\left(\left(\kappa^{\prime}\right)^{++}\right)^{Q^{\mathcal{T}}}$, so by 3.7 ,

$$
\left(\operatorname{Ult}\left(Q^{\mathcal{T}}, F^{\prime}\right) \sim B_{\beta+2}^{\mathcal{U}}\right) \|\left(\left(\gamma^{\mathcal{T}}\right)^{++}\right)^{\operatorname{Ult}\left(Q^{\mathcal{T}}, F^{\prime}\right)},
$$

and so $E_{\beta+2}^{\mathcal{U}}=F\left(N^{B^{\mathcal{T}}}\right)$ (the extender $E_{\beta+2}^{\mathcal{U}}$ must exist in the first place, by non-exactness). We leave the remaining details to the reader.

We can now complete the proof in the non-exceptional case:

Claim 5. If $B$ is non-exceptional then $m \geq 0$ and $B$ has a good core.

Proof. Suppose $B$ is non-exceptional. By the previous claim, $m \geq 0$ and we have an iterate $B^{\prime}$ of $B$ with a good core. But then the proof of Claim 7 of 4.3 shows that $B$ also has a good core.

We now prove corresponding claims for the exceptional case.

Claim 6. Suppose $B$ is exceptional. Then one of $B^{\mathcal{T}}, B^{\mathcal{U}}$ is a cephalanx with an exceptional core.

Proof. We first observe: 
SubClaim 6.1. Suppose the comparison terminates early (so $\alpha=\beta+1$ where $\beta$ is unusual). Then $\beta$ is type (ii), $B$ is exact, $S^{\mathcal{U}}=\{0\}=S^{\mathcal{T}}$, and the final models of the comparison are $M^{\mathcal{T}}=M^{\mathcal{U}}$.

Proof. The fact that $\beta$ is type (ii) and $B$ exact, is because $B$ is exceptional and Claim 1. So $\left(\rho^{+}\right)^{M^{\mathcal{T}}}=\left(\rho^{+}\right)^{M^{\mathcal{U}}}$ where $\rho=\rho^{\mathcal{T}}$, by Claim 1(5) (vi)(e). But $M^{\mathcal{T}}, M^{\mathcal{U}}$ project $\leq \rho$, so $M^{\mathcal{T}} \pitchfork M^{\mathcal{U}} \pitchfork M^{\mathcal{T}}$.

Now we consider a few cases:

CAse I. Either (a) $S^{\mathcal{T}}=\{0\}=S^{\mathcal{U}}$ and $M^{\mathcal{U}} \triangleleft M^{\mathcal{T}}$, or (b) $S^{\mathcal{T}}=\{0,1\}$.

This case is covered by the next case and symmetry.

CASE II. Either (a) $S^{\mathcal{T}}=\{0\}=S^{\mathcal{U}}$ and $M^{\mathcal{T}} \triangleleft M^{\mathcal{U}}$, or (b) $S^{\mathcal{U}}=\{0,1\}$.

Note that given either (a) or (b), $S^{\mathcal{T}}=\{0\}$ and $M^{\mathcal{T}} \triangleleft B^{\mathcal{U}}$. So $M^{\mathcal{T}}$ is sound, so $\alpha \in \mathscr{B}^{\mathcal{T}}$. Let $\beta=\operatorname{movin}^{\mathcal{T}}(\alpha)$. Because $\rho_{m+1}\left(M^{\mathcal{T}}\right) \leq \rho^{\mathcal{T}}$ and $M^{\mathcal{T}} \triangleleft B^{\mathcal{U}}, \mathcal{U}$ does not use any extender $E$ with $\rho^{\mathcal{T}}<\operatorname{lh}(E)$. So if $\beta$ is $(\mathcal{T}, \mathcal{U})$-unusual (so type (ii)) then $M^{\mathcal{T}} \triangleleft M_{\beta}^{\mathcal{U}}=M_{\beta+1}^{\mathcal{U}}=B^{\mathcal{U}}$ and $\alpha=\beta+1$, contradicting Subclaim 6.1. So (let) $E_{\beta}^{\mathcal{U}}=F^{\prime}=F\left(Q^{\mathcal{T}}\right)$. Similarly, $\beta+1$ is not $(\mathcal{U}, \mathcal{T})$-unusual. So by Claim 2 , if $\beta+1 \in \mathscr{B}^{\mathcal{U}}$ then $\rho^{\mathcal{T}}<\rho_{\beta+1}^{\mathcal{U}}$, so $B_{\beta+1}^{\mathcal{U}} \mid \rho^{\mathcal{T}}$ is well-defined. Let $\kappa=\operatorname{cr}\left(F^{\prime}\right)$ and $\varepsilon=\operatorname{pred}^{\mathcal{U}}(\beta+1)$. We split into two subcases:

SuBCASE II.1. $B_{\beta+1}^{\mathcal{U}} \mid \rho^{\mathcal{T}}$ is active.

Then $\beta+1 \notin \mathscr{B}^{\mathcal{U}}, B_{\beta+1}^{\mathcal{U}}$ is type $2, \rho^{\mathcal{T}}=\operatorname{OR}\left(B_{\beta+1}^{\mathcal{U}}\right),\left(\kappa^{+}\right)^{Q^{\mathcal{T}}}=\mathrm{OR}\left(B_{\beta+1}^{* \mathcal{U}}\right)$, $E_{\varepsilon}^{\mathcal{U}}=F\left(B_{\beta+1}^{* \mathcal{U}}\right), E_{\beta+1}^{\mathcal{U}}=F\left(B_{\beta+1}^{\mathcal{U}}\right)$, and $\alpha=\beta+2$. Let $R=B_{\beta+1}^{* \mathcal{U}}$ and $G=$ $F^{R}=E_{\varepsilon}^{\mathcal{U}}$ and $U=\operatorname{Ult}_{0}(R, G)$. Then $\left(\kappa^{+}\right)^{U}=\left(\kappa^{+}\right)^{Q^{\mathcal{T}}}=\mathrm{OR}^{R}$ and

$$
\left(U \sim Q^{\mathcal{T}}\right) \|\left(\kappa^{++}\right)^{Q^{\mathcal{T}}},
$$

but $\left(\kappa^{++}\right)^{U}>\left(\kappa^{++}\right)^{Q^{\mathcal{T}}}$ because $B^{\mathcal{T}}$ is exact and $M^{\mathcal{T}} \triangleleft B^{\mathcal{U}}$ and by 3.9 .

Let $H \triangleleft U$ and $h \in\{-1\} \cup \omega$ with $\rho_{h+1}^{H}=\left(\kappa^{+}\right)^{H}<\left(\kappa^{++}\right)^{H}=\left(\kappa^{++}\right)^{Q^{\mathcal{T}}} \leq \rho_{h}^{H}$. Let $H^{*}=i_{F^{\prime}}^{U}(H)$; so $H^{*} \triangleleft \operatorname{Ult}_{0}\left(U, F^{\prime}\right)$. Note $i_{F^{\prime}}^{U}$ is continuous at $\left(\kappa^{+}\right)^{Q^{\mathcal{T}}}$.

We claim that $\operatorname{Ult}_{h}\left(H, F^{\prime}\right) \unlhd H^{*}$. For if $h=-1$ then by continuity, in fact Ult $_{-1}\left(H, F^{\prime}\right)=H^{*}$, so suppose $h \geq 0$. Let

$$
\sigma: \operatorname{Ult}_{h}\left(H, F^{\prime}\right) \rightarrow H^{*}
$$

be the factor map $\sigma\left([a, f]_{F^{\prime}}^{H, h}\right)=i_{F^{\prime}}^{U}(f)(a)$. Arguing like in $\S 2$, we get that $H, \sigma \in \operatorname{Ult}_{0}\left(U, F^{\prime}\right)$ and the hypotheses of 2.13 hold for $H, \sigma, h, H^{*}$. By 2.13 (and its first order nature), $R \models$ "Lemma 2.13 holds for my proper segments". Therefore $\operatorname{Ult}_{h}\left(H, F^{\prime}\right) \unlhd H^{*}$, as desired.

So $\operatorname{Ult}_{h}\left(H, F^{\prime}\right) \triangleleft B_{\beta+2}^{\mathcal{U}}$. But $\left(\left(\rho^{\mathcal{T}}\right)^{+}\right)^{\mathrm{Ult}_{h}\left(H, F^{\prime}\right)}=\left(\left(\rho^{\mathcal{T}}\right)^{+}\right)^{M^{\mathcal{T}}}$, so $h=m$ and $M^{\mathcal{T}}=\operatorname{Ult}_{h}\left(H, F^{\prime}\right)$. It easily follows that $B^{\mathcal{T}}$ has an exceptional core, and with $X, m^{\prime}$ as in 4.13 ,

$$
H=\operatorname{cHull}_{m^{\prime}+1}^{M^{\mathcal{T}}}\left(X \cup z_{m+1}^{M^{\mathcal{T}}} \cup \vec{p}_{m}^{M^{\mathcal{T}}}\right) .
$$


SubCASE II.2. $B_{\beta+1}^{\mathcal{U}} \mid \rho^{\mathcal{T}}$ is passive.

Then $\alpha=\beta+1$, so $M^{\mathcal{T}} \triangleleft B_{\beta+1}^{\mathcal{U}}=B^{\mathcal{U}}$. Let $R=B_{\alpha}^{* \mathcal{U}}$. If $\alpha \in \mathscr{B}^{\mathcal{U}}$ then $\kappa<\gamma(R)$, so $\left(\kappa^{++}\right)^{R}$ is well-defined. In any case, $\kappa$ is not the largest cardinal of $R$. We have $\left(\kappa^{+}\right)^{R}=\left(\kappa^{+}\right)^{Q^{\mathcal{T}}}$ and $(R \sim Q) \|\left(\kappa^{++}\right)^{Q^{\mathcal{T}}}$. If $\left(\kappa^{++}\right)^{R}>\left(\kappa^{++}\right)^{Q^{\mathcal{T}}}$ then a simplification of the argument in the previous subcase works. Suppose then that $\left(\kappa^{++}\right)^{R}=\left(\kappa^{++}\right)^{Q^{\mathcal{T}}}$. Because $M^{\mathcal{T}} \triangleleft B^{\mathcal{U}}$, it is easy enough to see that $\alpha \notin \mathscr{B}^{\mathcal{U}}$, so $R$ is a premouse. If $R$ is active type 3 , then $\left(\kappa^{+}\right)^{R}<\nu\left(F^{R}\right)$, because if $\left(\kappa^{+}\right)^{R}=\nu\left(F^{R}\right)$ then $\operatorname{OR}\left(B_{\beta+1}^{\mathcal{U}}\right)=\left(\left(\rho^{\mathcal{T}}\right)^{+}\right)^{M^{\mathcal{T}}}$, a contradiction. Let $d=\operatorname{deg}^{\mathcal{U}}(\beta+1)$. Then $i_{\beta+1}^{* \mathcal{U}}$ is discontinuous at $\left(\kappa^{++}\right)^{R}$, and so $\left(\kappa^{+}\right)^{R}=\rho_{d}^{R}$, so $d>0$. Let $r<d$ be such that $\rho_{r+1}^{R}=\left(\kappa^{+}\right)^{R}<\rho_{r}^{R}$. Then arguing like in the previous subcase, but using 3.28 instead of 2.13,

$$
M^{\mathcal{T}}=\mathrm{Ult}_{r}\left(R, F^{\prime}\right) \triangleleft B_{\beta+1}^{\mathcal{U}}
$$

and $B^{\mathcal{T}}$ has an exceptional core (with $m=r$ ).

CASE III. $S^{\mathcal{T}}=\{0\}=S^{\mathcal{U}}$ and $M^{\mathcal{T}}=M^{\mathcal{U}}$ but the comparison does not terminate early.

Then $\alpha \in \mathscr{B}^{\mathcal{T}} \Delta \mathscr{B}^{\mathcal{U}}$; assume $\alpha \in \mathscr{B}^{\mathcal{T}} \backslash \mathscr{B}^{\mathcal{U}}$. Let $\beta=\operatorname{movin}^{\mathcal{T}}(\alpha)$.

SubClaim $6.2 . \beta$ is not $(\mathcal{T}, \mathcal{U})$-unusual.

Proof. Suppose otherwise, so $\beta$ is type (ii) and $E_{\beta}^{\mathcal{T}}=E_{\beta}^{\mathcal{U}}=\emptyset$. Since the comparison does not terminate early and $M^{\mathcal{T}}$ is $\rho^{\mathcal{T}}$-sound, we have $E_{\beta+1}^{\mathcal{U}} \neq \emptyset=$ $E_{\beta+1}^{\mathcal{T}}$ and $\alpha=\beta+2$ and

$$
\rho^{\mathcal{T}}=\nu_{\beta}^{\mathcal{U}}<\operatorname{lh}_{\beta}^{\mathcal{U}}=\left(\left(\rho^{\mathcal{T}}\right)^{+}\right)^{M^{\mathcal{T}}} .
$$

So $\rho^{\mathcal{T}}$ is not the largest cardinal in $M^{\mathcal{T}}$, so is also not in $M_{\beta}^{\mathcal{U}}$. So $\operatorname{ex}_{\beta}^{\mathcal{U}} \triangleleft M_{\beta}^{\mathcal{U}}$, so $\left(\left(\rho^{\mathcal{T}}\right)^{+}\right)^{M^{\mathcal{T}}}<\left(\left(\rho^{\mathcal{T}}\right)^{+}\right)^{M_{\beta}^{\mathcal{U}}}$, contradicting Claim 1(5) (vi)(e).

So $E_{\beta}^{\mathcal{U}}=F^{\prime}=F\left(Q^{\mathcal{T}}\right)$.

Subclaim 6.3. $\beta+1$ is not $(\mathcal{U}, \mathcal{T})$-unusual.

Proof. This is like the proof of Subclaim 6.2.

By the subclaim and Claim 2, (1) if $\beta+1 \in \mathscr{B}^{\mathcal{U}}$ then $\rho^{\mathcal{T}}<\rho_{\beta+1}^{\mathcal{U}}$, and (2) one of the following holds:

(a) $\alpha=\beta+1$.

(b) $\alpha=\beta+2$ and $\operatorname{lh}_{\beta+1}^{\mathcal{U}}=\rho^{\mathcal{T}}$ and $E_{\beta+1}^{\mathcal{U}}$ is type 2 .

(c) $\alpha=\beta+2, \operatorname{lh}_{\beta+1}^{\mathcal{U}}=\left(\left(\rho^{\mathcal{T}}\right)^{+}\right)^{M^{\mathcal{T}}}$ and $E_{\beta+1}^{\mathcal{U}}$ is (i) type 1 or (ii) type 3 .

(d) $\alpha=\beta+3, \operatorname{lh}_{\beta+1}^{\mathcal{U}}=\rho^{\mathcal{T}}, E_{\beta+1}^{\mathcal{U}}$ is type $2, \operatorname{lh}_{\beta+2}^{\mathcal{U}}=\left(\left(\rho^{\mathcal{T}}\right)^{+}\right)^{M^{\mathcal{T}}}$ and $E_{\beta+2}^{\mathcal{U}}$ is (i) type 1 or (ii) type 3 . 
The same general argument works in each case, but the details vary. We just discuss cases (a), (b), (c)(i) and sketch (d)(i). In each case let $\varepsilon=\operatorname{pred}^{\mathcal{U}}(\beta+1)$ and $R=B_{\beta+1}^{* \mathcal{U}}$ and $\kappa=\operatorname{cr}\left(F^{\prime}\right)$. Note that if $m=-1$ then case (a) attains.

Consider case (a). Since $S^{\mathcal{U}}=\{0\}, R$ is a premouse. Let $d=\operatorname{deg}^{\mathcal{U}}(\alpha)$. So $R$ is $d$-sound and $M^{\mathcal{U}}=\operatorname{Ult}_{d}\left(R, F^{\prime}\right)$. Clearly $d \geq m$. We claim that $d>m$ (so $R$ is $(m+1)$-sound) and $\rho_{m+1}^{R}=\left(\kappa^{+}\right)^{R}<\rho_{m}^{R}$. For if $\rho_{m+1}^{R}>\left(\kappa^{+}\right)^{R}$ then

$$
\rho_{m+1}\left(M^{\mathcal{U}}\right)>\rho^{\mathcal{T}} \geq \rho_{m+1}\left(M^{\mathcal{T}}\right)
$$

and if $\rho_{m+1}^{R} \leq \kappa$ then $\rho_{m+1}\left(M^{\mathcal{U}}\right)<\rho^{\mathcal{T}}$ and $M^{\mathcal{U}}$ is $\gamma^{\mathcal{T}}$-sound, so $B^{\mathcal{T}}$ is not exceptional, contradicting 4.12.

Let $U_{i}=\operatorname{Ult}_{i}\left(R, F^{\prime}\right)$, so $M^{\mathcal{U}}=U_{d}$. Note $\kappa^{++R}=\kappa^{++B^{\mathcal{T}}}$ and $\left(\rho^{\mathcal{T}}\right)^{+U_{m}}=$ $\left(\rho^{\mathcal{T}}\right)^{+M^{\mathcal{T}}}$, but $\rho_{m+1}^{U_{m}}=\rho^{\mathcal{T}}$, so $U_{m} \notin M^{\mathcal{T}}=U_{d}$, so arguing like in the proof of 2.13, $U_{m}=U_{d}$ and the factor map $\sigma: U_{m} \rightarrow U_{d}$ is the identity (this does not use condensation). Letting $\pi=i_{F^{\prime}}^{R, m}$ and $H=R$, then $H, \pi$ are as in 4.13.

Now consider case (b). Note that $R=\operatorname{ex}_{\varepsilon}^{\mathcal{U}}$, is active type 2 and $\mathrm{OR}^{R}=$ $\left(\kappa^{+}\right)^{B^{\mathcal{T}}}$. Note that $\operatorname{deg}^{\mathcal{U}}(\beta+2)=m$ and $\operatorname{cr}\left(F^{R}\right)=\operatorname{cr}_{\beta+1}^{\mathcal{U}}$, so $\operatorname{pred}^{\mathcal{U}}(\beta+2)=$ $\operatorname{pred}^{\mathcal{U}}(\varepsilon+1)$ and $B_{\varepsilon+1}^{* \mathcal{U}}=B_{\beta+2}^{* \mathcal{U}}$ and $\operatorname{deg}^{\mathcal{U}}(\varepsilon+1)=m$. Let $H=B_{\varepsilon+1}^{\mathcal{U}}$. Then $\operatorname{Ult}_{m}\left(H, F^{\prime}\right)=M^{\mathcal{T}}$ and letting $\pi=i_{F^{\prime}}^{H, m}$, then $H, \pi$ are as in 4.13.

Now consider case (c)(i).

Subclaim 6.4. In case (c)(i), $E_{\varepsilon}^{\mathcal{U}}$ is the "preimage" of $E_{\beta+1}^{\mathcal{U}}$ under $i_{\beta+1}^{* \mathcal{U}}$ and $\operatorname{lh}_{\varepsilon}^{\mathcal{U}}=\left(\kappa^{++}\right)^{B^{\mathcal{T}}}$.

Proof. We have $\mathrm{ex}_{\varepsilon}^{\mathcal{U}} \unlhd R$ and $\left(\kappa^{+}\right)^{B^{\mathcal{T}}}=\left(\kappa^{+}\right)^{\operatorname{ex}_{\beta}^{\mathcal{U}}}=\left(\kappa^{+}\right)^{R}=\left(\kappa^{+}\right)^{\operatorname{ex}_{\varepsilon}^{\mathcal{U}}}<\operatorname{lh}_{\varepsilon}^{\mathcal{U}}$. We have $\left(\kappa^{++}\right)^{R} \geq\left(\kappa^{++}\right)^{B^{\mathcal{T}}}$ and if $\beta+1 \in \mathscr{B}^{\mathcal{U}}$ then $\left(\kappa^{++}\right)^{R}>\left(\kappa^{++}\right)^{B^{\mathcal{T}}}$; the latter is because $\operatorname{ex}_{\beta+1}^{\mathcal{U}} \not \Perp M^{\mathcal{T}}$ and $\operatorname{ex}_{\beta+1}^{\mathcal{U}}$ projects $\leq \rho^{\mathcal{T}}$. Let $P \unlhd R$ and $p \in\{-1\} \cup \omega$ with

$$
\rho_{p+1}^{P} \leq\left(\kappa^{+}\right)^{B^{\mathcal{T}}}<\left(\kappa^{++}\right)^{B^{\mathcal{T}}}=\left(\kappa^{++}\right)^{P} \leq \rho_{p}^{P}
$$

(so $P$ is $p$-sound). By condensation, like before, $U^{P, p}=\operatorname{Ult}_{p}\left(P, F^{\prime}\right) \unlhd M_{\beta+1}^{\mathcal{U}}$. But $\left(\left(\rho^{\mathcal{T}}\right)^{+}\right)^{U^{P, p}}=\left(\left(\rho^{\mathcal{T}}\right)^{+}\right)^{M^{\mathcal{T}}}$, and as $\nu_{\beta+1}^{\mathcal{U}}=\rho^{\mathcal{T}}$, therefore $U^{P, p}=\operatorname{ex}_{\beta+1}^{\mathcal{U}}$.

So $P$ is type $1, p=0, \mathrm{OR}^{P}=\left(\kappa^{++}\right)^{B^{T}}$, and $E_{\varepsilon}^{\mathcal{U}}=F^{P}$. Now $i_{\beta+1}^{* \mathcal{U}}$ is continuous at $\left(\kappa^{+}\right)^{B^{\tau}}$. So if $P \triangleleft R$ then $i_{\beta+1}^{* \mathcal{U}}$ is continuous at $\operatorname{OR}^{P}$, so $i_{\beta+1}^{* \mathcal{U}}(P)=$ $\operatorname{ex}_{\beta+1}^{\mathcal{U}}\left(\right.$ or $\psi_{j}(P)=\operatorname{ex}_{\beta+1}^{\mathcal{U}}$ where $\left.j=i_{\beta+1}^{* \mathcal{U}}\right)$. If $P=R$ then $\operatorname{Ult}_{p}\left(P, F^{\prime}\right)=M_{\beta+1}^{\mathcal{U}}$ (even if $0<\operatorname{deg}^{\mathcal{U}}(\beta+1)$ ).

Since $E_{\varepsilon}^{\mathcal{U}}=F^{P}$ and $\operatorname{cr}\left(F^{P}\right)=\operatorname{cr}\left(F^{\prime}\right)$, $\operatorname{pred}^{\mathcal{U}}(\varepsilon+1)=\varepsilon$ and $B_{\varepsilon+1}^{* \mathcal{U}}=R$ and $\operatorname{deg}^{\mathcal{U}}(\varepsilon+1)=\operatorname{deg}^{\mathcal{U}}(\beta+1)$. Also, $\operatorname{pred}^{\mathcal{U}}(\beta+2)=\beta+1$ and $m=\operatorname{deg}^{\mathcal{U}}(\beta+2)=$ $\operatorname{deg}^{\mathcal{U}}(\varepsilon+1)$. Using this, and letting $H=B_{\varepsilon+1}^{\mathcal{U}}$, we get $\operatorname{Ult}_{m}\left(H, F^{\prime}\right)=M^{\mathcal{T}}$ and letting $\pi=i_{F^{\prime}}^{H, m}$, then $H, \pi$ are as in 4.13.

Finally consider case (d)(i). For illustration, assume that $\beta+2 \notin \mathscr{B}^{\mathcal{U}}$. Let $\chi=\operatorname{pred}^{\mathcal{U}}(\beta+2)$ and $S=B_{\beta+2}^{* \mathcal{U}}$ and $c=\operatorname{deg}^{\mathcal{U}}(\beta+2)$. A combination of the preceding arguments gives the following: 
- $\operatorname{ex}_{\varepsilon}^{\mathcal{U}}$ is the (type 2) preimage of $\operatorname{ex}_{\beta+1}^{\mathcal{U}}$ under $i_{\beta+1}^{* \mathcal{U}}$,

$-\operatorname{pred}^{\mathcal{U}}(\varepsilon+1)=\chi$ and $B_{\varepsilon+1}^{* \mathcal{U}}=S$ and $\operatorname{deg}^{\mathcal{U}}(\varepsilon+1)=c$,

- $\operatorname{ex}_{\varepsilon+1}^{\mathcal{U}}$ is the (type 1) preimage of $\operatorname{ex}_{\beta+2}^{\mathcal{U}}$ under the map $\sigma$ defined below,

- $\varepsilon=\operatorname{pred}^{\mathcal{U}}(\varepsilon+2)$ and $\operatorname{deg}^{\mathcal{U}}(\varepsilon+2)=0$,

$-\beta+1=\operatorname{pred}^{\mathcal{U}}(\beta+3)$ and $m=\operatorname{deg}^{\mathcal{U}}(\beta+3)=0$.

Let $J=B_{\varepsilon+1}^{\mathcal{U}}$ and $H=B_{\varepsilon+2}^{\mathcal{U}}$. Then also, $\operatorname{Ult}_{c}\left(J, F^{\prime}\right)=B_{\beta+2}^{\mathcal{U}}$ and letting $\sigma=i_{F^{\prime}}^{J, c}$, then $\sigma\left(\operatorname{ex}_{\varepsilon+1}^{\mathcal{U}}\right)=\operatorname{ex}_{\beta+2}^{\mathcal{U}}$ (as mentioned above), and $\operatorname{Ult}_{0}\left(H, F^{\prime}\right)=M^{\mathcal{T}}$, etc.

Cases (c)(ii) and (d)(ii) are fairly similar to the preceding cases. However, while in the preceding cases there is always some $\zeta<\rho^{\mathcal{T}}$ such that

$$
M^{\mathcal{T}}=\operatorname{Hull}_{m+1}^{M^{\mathcal{T}}}\left(\zeta \cup\left\{\vec{p}_{m}^{M}, z_{m+1}^{M}\right\}\right),
$$

there is no such $\zeta$ in (c)(ii) and (d)(ii).

There is just one case left:

CASE IV. The comparison terminates early (so by Subclaim $6.1, S^{\mathcal{T}}=\{0\}=S^{\mathcal{U}}$ and $M^{\mathcal{T}}=M^{\mathcal{U}}$ ).

We may assume that $\alpha$ is $(\mathcal{T}, \mathcal{U})$-unusual (type (ii)). Let $\xi_{0}<\xi_{1}<\alpha=\xi_{1}+1$ witness this. So $E_{\xi_{1}}^{\mathcal{T}}=F^{\prime}=F\left(Q^{\mathcal{U}}\right)$. We have $M^{\mathcal{U}}=M^{\mathcal{T}}$. Let $H=M_{\xi_{0}}^{\mathcal{T}}$. Then $\operatorname{Ult}_{m}\left(H, F^{\prime}\right)=M^{\mathcal{T}}=M^{\mathcal{U}}$ etc.

Since we now have an iterate $B^{\prime}$ of $B$ with an exceptional core, the next claim completes the proof of the theorem:

Claim 7. Suppose that $B$ is exceptional and let $B^{\prime}$ be a cephalanx non-dropping iterate of $B$. If $B^{\prime}$ has an exceptional core then so does $B$.

Proof. The proof is similar to 4.3, but with some extra argument. We assume that $m \geq 0$ and leave the other case to the reader (the main distinction in that case is that even though $m=-1$, all ultrapower embeddings are at least $\mathrm{r} \Sigma_{1}$ elementary). Fix $H, \kappa, F, X$ and $\pi: H \rightarrow M$ as in 4.13. Let $B^{\prime}=\left(\gamma^{\prime}, \rho^{\prime}, M^{\prime}, Q^{\prime}\right)$ and fix $H^{\prime}, \kappa^{\prime}, F^{\prime}, X^{\prime}, \pi^{\prime}$ as in 4.13 for $B^{\prime}$. Suppose $B^{\prime}$ has an exceptional core. Let $i: M \rightarrow M^{\prime}$ and $j: Q \rightarrow Q^{\prime}$ be the iteration maps. So $j=i \uparrow\left(B \| \gamma^{+M}\right)$. Note $i\left(\kappa, \vec{p}_{m}^{M}, z_{m+1}^{M}\right)=\left(\kappa^{\prime}, \vec{p}_{m}^{M^{\prime}}, z_{m+1}^{M^{\prime}}\right)$, and for $\alpha<\gamma^{+M}$, we have $X \cap \alpha \in$ $B \| \gamma^{+M}$ and

$$
i(X \cap \alpha)=X^{\prime} \cap i(\alpha) .
$$

From these facts, and because $X^{\prime}=\left(\gamma^{\prime}\right)^{+M^{\prime}} \cap \operatorname{rg}\left(\pi^{\prime}\right)$, it is easy to see that $X=\gamma^{+M} \cap \operatorname{rg}(\pi)$. It remains to see $H \| \kappa^{++H}=M \mid \kappa^{++M}$.

Let $Y=\operatorname{rg}(\pi) \cap \gamma^{++M}$. Let $\sigma=i_{F}^{M}$ and $Z=\operatorname{rg}(\sigma) \cap \gamma^{++M}$. It suffices to see that $Y=Z$. Let $Y^{\prime}, \sigma^{\prime}, Z^{\prime}$ be defined analogously from $B^{\prime}$. Because $B^{\prime}$ has an exceptional core, Lemma 4.14 applies, and $Y^{\prime}=Z^{\prime}$ follows. We will use this to deduce that $Y=Z$, by breaking $Y$ and $Z$ into unions of small pieces, and considering how they move under the iteration map $i$. 
SubClaim 7.1. For any $\alpha<\gamma^{++M}$, we have $\alpha \in Y$ iff $i(\alpha) \in Y^{\prime}$.

Proof. If $\alpha \in Y$ then $i(\alpha) \in Y^{\prime}$ because $i^{\prime \prime} X \subseteq X^{\prime}$ and $i\left(z_{m+1}^{M}\right)=z_{m+1}^{M^{\prime}}$.

Suppose $\alpha \notin Y$. For $\beta<\gamma^{+M}$ and $\delta<\rho_{m}^{M}$ let $Y_{\beta, \delta}$ be the set of all $\xi<\gamma^{++M}$ such that

$$
\xi \in \operatorname{Hull}_{m+1}^{M}\left((X \cap \beta) \cup z_{m+1}^{M} \cup \vec{p}_{m}^{M}\right),
$$

as witnessed by some theory below $\operatorname{Th}_{\mathrm{r} \Sigma}^{M}\left(\delta \cup\left\{\vec{p}_{m}^{M}\right\}\right)$. (See [3, $\left.\S 2\right]$, in particular, the stratification of $\mathrm{r} \Sigma_{m+1}$ described there, for more details. If $m=0$ this needs to be interpreted appropriately; for example, if $M$ is passive and $\mathrm{OR}^{M}$ is divisible by $\omega^{2}$, that the $\mathrm{r} \Sigma_{1}$ fact should hold in $M \mid \delta$.) Then $Y_{\beta, \delta} \in M$. Define $Y_{\beta, \delta}^{\prime}$ analogously over $M^{\prime}$. Let $I=\rho_{m}^{M} \times \gamma^{+M}$. Using line (7), we get $i\left(Y_{\beta, \delta}\right)=Y_{i(\beta), i(\delta)}^{\prime}$, and note $Y^{\prime}=\bigcup_{(\beta, \delta) \in I} i\left(Y_{\beta, \delta}\right)$. The fact that $i(\alpha) \notin Y^{\prime}$ follows easily.

SubClaim 7.2. For any $\alpha<\gamma^{++M}$, we have $\alpha \in Z$ iff $i(\alpha) \in Z^{\prime}$.

Proof. Let $\alpha<\gamma^{++M}$. Let $\beta<\kappa^{++M}$ with $\alpha<\sigma(\beta)$. Fix a surjection $f: \kappa^{+M} \rightarrow \beta$ in $M$. So $\sigma(f): \gamma^{+M} \rightarrow \sigma(\beta)$ is a surjection in $M$, and note that $\operatorname{rg}(\sigma) \cap \sigma(\beta)=\sigma(f)$ " $X$.

Now we claim that $i(\sigma(f))=\sigma^{\prime}(i(f))$. For let $C$ be the prewellorder of $\kappa^{+M}$ corresponding to $f$ (so $(\delta, \varepsilon) \in C$ iff $f(\delta) \leq f(\varepsilon)$ ). Then it suffices to see that $i(\sigma(C))=\sigma^{\prime}(i(C))$. But this holds by continuity at $\kappa^{+M}$ and because $i(\sigma(D))=\sigma^{\prime}(i(D))$ for all $D \in \mathcal{P}(\kappa) \cap M$.

So let $f^{\prime}=i(f)$ and $\beta^{\prime}=i(\beta)=\operatorname{rg}\left(f^{\prime}\right)$. Then $\sigma^{\prime}\left(\beta^{\prime}\right)=\operatorname{rg}(i(\sigma(f)))$, so $i(\alpha)<\sigma^{\prime}\left(\beta^{\prime}\right)$. Therefore $i(\alpha) \in Z^{\prime}$ iff $i(\alpha) \in \sigma^{\prime}\left(f^{\prime}\right)$ " $X^{\prime}$ iff $i(\alpha) \in i(\sigma(f))$ " $X^{\prime}$.

But we have $X^{\prime}=\bigcup_{\delta<\gamma^{+M}} i(X \cap \delta)$. So $\alpha \in Z$ iff $\alpha \in \sigma(f)$ " $X$ iff there is $\delta<\gamma^{+M}$ such that $\alpha \in \sigma(f)$ " $(X \cap \delta)$ iff there is $\delta^{\prime}<i(\gamma)^{+M^{\prime}}$ such that $i(\alpha) \in i(\sigma(f))$ " ( $\left(X^{\prime} \cap \delta^{\prime}\right)$ iff $i(\alpha) \in i(\sigma(f))$ " $X^{\prime}$ iff $i(\alpha) \in Z^{\prime}$, as desired.

Clearly by the subclaims, we have $Y=Z$, as desired.

This completes the proof of the claim, and hence the theorem.

\section{Condensation from solidity and normal iterability}

By $(k+1)$-condensation ${ }^{29}$, if $H, M$ are $(k+1)$-sound premice such that $M$ is $\left(k, \omega_{1}, \omega+1\right)^{*}$-iterable and $\pi: H \rightarrow M$ a near $k$-embedding with $\operatorname{cr}(\pi) \geq \rho$ where $\rho=\rho_{k+1}^{H}$, then (*Con) either $H \unlhd M$ or $\left[M \mid \rho\right.$ is active and $\left.H \triangleleft \operatorname{Ult}\left(M \mid \rho, F^{M \mid \rho}\right)\right]$.

We now prove that $\left(k, \omega_{1}+1\right)$-iterability suffices for this result. In our proof, we will replace the phalanx used in the standard proof with a cephal, and avoid Dodd-Jensen. We will in fact prove a partial analogue of the more refined version [18, Theorem 9.3.2] (but for Mitchell-Steel indexing, with superstrongs).

${ }^{29}$ Cf. [3, pp. $\left.87-88\right]$ or [18, Theorem 9.3.2]. 
We do not achieve a full analogue here, because in the case that $H \notin M$ we encounter an obstacle in connection with exceptional cephalanxes. So in this sense we do not quite prove full condensation. However, if assume also that $M$ is $(k+1)$-solid, we can deduce the full analogous conclusion. ${ }^{30}$

5.1 Definition. Let $M$ be a $k$-sound premouse and $\zeta_{k+1}^{M} \leq \rho \leq \rho_{k}^{M}$. The $\rho$-solid-core of $M$ is $H=\operatorname{cHull}_{k+1}^{M}\left(\rho \cup z_{k+1}^{M} \cup \vec{p}_{k}^{M}\right)$, and the $\rho$-solid-core map is the uncollapse map $\pi: H \rightarrow M$.

The $\rho$-solid-core map is a $k$-embedding, since $H \notin M$ and by 2.4 .

5.2 Theorem (Condensation from solidity). Let $M$ be a $k$-sound, $\left(k, \omega_{1}+1\right)$ iterable premouse. Let $H$ be a $\rho$-sound premouse with $\rho \in\left[\rho_{k+1}^{H}, \rho_{k}^{H}\right)$ an $H$ cardinal; let $\gamma=\operatorname{card}^{M}(\rho)$. Let $\pi: H \rightarrow M$ be $k$-lifting with $\operatorname{cr}(\pi) \geq \rho$. Then:

1. If $H \notin M$ then:

(a) $\zeta_{k+1}^{H}=\zeta_{k+1}^{M} \leq \rho$ and $\pi\left(z_{k+1}^{H}\right)=z_{k+1}^{M}$,

(b) $H$ is the $\rho$-solid-core of $M$ and $\pi$ is the $\rho$-solid-core map,

(c) $\rho_{k+1}^{H} \notin[\gamma, \rho)$,

(d) if $\rho_{k+1}^{H}=\rho$ and $\rho^{+H}<\rho^{+M}$ then $M \mid \rho$ is active with a superstrong extender with critical point $\kappa$ and $\rho_{k+1}^{M} \leq\left(\kappa^{+}\right)^{M}<\rho$,

(e) $\rho_{k+1}^{H} \geq \rho_{k+1}^{M}$,

(f) if $M$ is $(k+1)$-solid then $\rho_{k+1}^{H}=\rho_{k+1}^{M}$,

(g) if $\rho_{k+1}^{H}=\rho_{k+1}^{M}$ then $H$ is the $\rho$-core of $M, \pi$ is the $\rho$-core map and $\pi\left(p_{k+1}^{H}\right)=p_{k+1}^{M}$.

2. If $H \in M$ then exactly one of the following holds:

(a) $H \triangleleft M$, or

(b) $M \mid \rho$ is active with extender $F$ and $H \triangleleft \operatorname{Ult}(M \mid \rho, F)$, or

(c) $M \mid \rho$ is passive, $N=M \mid \rho^{+H}$ is active type 1 and $H=\operatorname{Ult}_{k}\left(Q, F^{N}\right)$, where $Q \triangleleft M$ is such that $\gamma^{+Q}=\rho$ and $\rho_{k+1}^{Q}=\gamma<\rho_{k}^{Q}$, or

(d) $k=0$ and $H, M$ are active type 2 and $M \mid \rho$ is active with a type 2 extender $F$ and letting $R=\operatorname{Ult}(M \mid \rho, F)$, then $N=R \mid \rho^{+H}$ is active type 1 and $H=\operatorname{Ult}_{0}\left(M \mid \rho, F^{N}\right)$.

5.3 Remark. If we assume further that $H, M$ are $(k+1)$-sound, it is now easy to conclude that $(*$ Con) (stated above) holds. In fact, it suffices to assume that if $H \notin M$ then $M$ is $\rho$-sound, and if $H \in M$ then $H$ is $(k+1)$-sound.

Proof.

\footnotetext{
${ }^{30}$ It will in fact be shown in [7] that $M$ is $(k+1)$-solid (from $\left(k, \omega_{1}+1\right)$-iterability), so the two papers together will prove the full result.
} 
Claim 1. We may assume:

- $H, M$ have the same type,

- if $H, M$ are passive and $k=0$ then $\pi$ is cofinal, and hence

$-\pi$ is c-preserving.

Proof. Suppose that $H, M$ have different types. Then $k=0, H$ is passive and $M$ is active. Here $M$ might an unsquashed or squashed premouse. In either case, note that $\pi: H \rightarrow M^{\prime}=M \| \mathrm{OR}^{M}$ is still 0 -lifting, and $M^{\prime}$ is $\omega$-sound with $\rho_{\omega}^{M^{\prime}}=\mathrm{OR}^{M^{\prime}}$. If part (2) (of the conclusion of Theorem 5.2) holds for $\pi: H \rightarrow M^{\prime}$, then it also holds for $\pi: H \rightarrow M$, so we are done. So suppose part (1) holds for $\pi: H \rightarrow M^{\prime}$. Then because $M^{\prime}$ is 1 -sound, by parts (1)(f),(g), in fact $H=M^{\prime}$ and $\pi=$ id. But $\rho_{1}^{H}<\rho_{0}^{H}$, contradicting that $\rho_{\omega}^{M^{\prime}}=\mathrm{OR}^{M^{\prime}}$.

Now suppose that $H, M$ are passive and $k=0$ but $\pi$ " $\mathrm{OR}^{H}$ is bounded in $\mathrm{OR}^{M}$. Let $M^{\prime}=M \| \sup \pi$ "OR ${ }^{H}$. Note that $\pi: H \rightarrow M^{\prime}$ is also 0-lifting, and is cofinal in $\mathrm{OR}^{M^{\prime}}$, and $M^{\prime}$ is $\omega$-sound. And $M^{\prime} \triangleleft M$, since otherwise $M \mid \mathrm{OR}^{M^{\prime}}$ is active, but then $M^{\prime} \models \mathrm{ZFC}^{-}$, which contradicts the fact that

$$
\operatorname{rg}(\pi)=\operatorname{Hull}_{1}^{M^{\prime}}\left(\rho \cup\left\{\pi\left(p_{1}^{H}\right)\right\}\right)
$$

is cofinal in $M^{\prime}$. Again if part (2) holds for $\pi: H \rightarrow M^{\prime}$, then we are done, so suppose part (1) holds. As $M^{\prime}$ is 1-sound, then $H=M^{\prime}$ and $\pi=$ id, so $H \triangleleft M$, so we are done.

From now on we make the assumptions stated in Claim 1. Using 2.4, we get: Claim 2. If $\rho^{+H}=\rho^{+M}$ or $\rho_{k+1}^{H}<\gamma$ then $H \notin M$ and $\pi$ is a $k$-embedding.

An easy calculation using the $\rho$-soundness of $H$ gives (cf. [12, 2.17]):

Claim 3. $\zeta_{k+1}^{H} \leq \rho$ and $p_{k+1}^{H} \backslash \rho=z_{k+1}^{H} \backslash \rho$.

Claim 4. If $H \notin M$ and (1)(a),(c) hold then so do (1)(b), (e), (f),(g).

Proof. $\pi$ is a $k$-embedding. So (b) follows from Claim 3 and (a). Part (e): Let $\kappa=\rho_{k+1}^{H}$. If $\mathcal{P}(\kappa)^{H}=\mathcal{P}(\kappa)^{M}$ then (e) is clear. If $\mathcal{P}(\kappa)^{H} \neq \mathcal{P}(\kappa)^{M}$ then by (c), $\kappa=\rho$, so because $H \notin M$, (e) holds. Part (f): As $\rho_{k+1}^{M} \leq \rho_{k+1}^{H}$, we get $\rho_{k+1}^{M}=\rho_{k+1}^{H}$ by $[12,2.17]$ and (a). Part (g): Suppose $\rho_{k+1}^{M}=\rho_{k+1}^{H}$. We have $\rho_{k+1}^{H} \leq \rho$. If $\rho_{k+1}^{M}=\rho_{k+1}^{H}=\rho$ then as $H \notin M$, and by the solidity of $p_{k+1}^{H}=p_{k+1}^{H} \backslash \rho$, we then have $p_{k+1}^{M}=\pi\left(p_{k+1}^{H}\right)$. Suppose $\rho_{k+1}^{M}=\rho_{k+1}^{H}=\kappa<\rho$, so by (c), $\kappa<\gamma$. So $\mathcal{P}(\kappa)^{M}=\mathcal{P}(\kappa)^{H}$, so $p_{k+1}^{M} \leq \pi\left(p_{k+1}^{H}\right)$, and so using the solidity of $p_{k+1}^{H} \backslash \rho$, we get $\pi\left(p_{k+1}^{H} \backslash \rho\right)=p_{k+1}^{M} \backslash \rho$, and since $\pi \uparrow \rho=\mathrm{id}$, we get $\pi\left(p_{k+1}^{H}\right)=p_{k+1}^{M}$. Now (g) easily follows.

There are two main cases overall. CASE 1. $\rho^{+H}=\rho^{+M}$.

We show (1). It suffices to prove (1) (a),(c), (d), by Claim 4. Part (d) is trivial by case hypothesis. By claims above, $H \notin M, \pi$ is a $k$-embedding, and 
$\zeta_{k+1}^{H} \leq \rho$. Using generalized solidity witnesses and as $\mathcal{P}(\rho)^{H}=\mathcal{P}(\rho)^{M}$, (a) follows. For part (c), we show $\gamma=\rho$. Suppose $\gamma<\rho$. So $\rho$ is an $H$-cardinal but non- $M$-cardinal, and $\rho \leq \operatorname{cr}(\pi)$. So $\rho=\gamma^{+H}$ and $\pi(\rho)=\gamma^{+M}=\rho^{+M}$. But as $\rho^{+H}=\rho^{+M}$ this contradicts condensation for $\omega$-sound mice (2.13).

CASE 2. $\rho^{+H}<\rho^{+M}$.

Let $\eta=\rho^{+H}$. Either (I) $\operatorname{cr}(\pi)=\rho$, or (II) $\operatorname{cr}(\pi)=\eta<\rho_{0}^{H}$, or (III) $\eta=\rho_{k}^{H}=\rho_{0}^{H}$ and $\operatorname{cr}(\pi)$ does not exist.

Assume (III) holds. Then by Claim 1 and case hypothesis, $H, M$ are active and $\rho$ is an $M$-cardinal. Letting $\mu=\operatorname{cr}\left(F^{H}\right)$, then $\operatorname{cr}\left(F^{M}\right)=\mu<\rho$ and $\left(\mu^{+}\right)^{H}=\left(\mu^{+}\right)^{M}$ and $\pi \uparrow\left(\mu^{+}\right)^{H}=$ id. It follows that $H, M$ are type 3 (consider the amenable predicate $E^{H}$ coding $F^{H}$ and its cofinality in $\left(H \mid\left(\mu^{+}\right)^{H}\right) \times$ $H \| \mathrm{OR}^{H}$, and likewise for $M$; we get $E^{H} \subseteq E^{M}$, and a contradiction to the fact that $\left.\mathrm{OR}^{H}<\mathrm{OR}^{M}\right)$. So $\eta=\rho_{k}^{H}=\rho_{0}^{H}=\nu\left(F^{H}\right)<\nu\left(F^{M}\right)$, and $F^{H}\left\lceil\eta \subseteq F^{M}\right.$. So by the ISC, if $M \mid \eta$ is passive then $H \triangleleft M$, and if $M \mid \eta$ is active then $H \triangleleft \operatorname{Ult}\left(M \mid \eta, F^{M \mid \eta}\right)$, but as $\rho_{k+1}^{H}<\eta$, the latter is impossible.

From now on we assume either (I) or (II) holds, so $\operatorname{cr}(\pi)$ exists. We will produce an iterable cephal $C$ and use it to deduce the required facts. If $M \mid \rho$ is passive then let $J \triangleleft M$ be least with $\rho_{\omega}^{J} \leq \rho$ and $\eta \leq \mathrm{OR}^{J}$. If $M \mid \rho$ is active and $\eta<\rho^{+U_{\rho}}$ where $U_{\rho}=\operatorname{Ult}\left(M \mid \rho, F^{M \mid \rho}\right)$, let $J \triangleleft U_{\rho}$ be least with $\eta \leq \mathrm{OR}^{J}$ and $\rho_{\omega}^{J}=\rho$. Otherwise leave $J$ undefined. We may assume $H \neq J$ (otherwise (2) holds). This ensures the cephal $C$ defined next is non-trivial.

If $\rho$ is an $M$-cardinal, let $C=(\rho, H, J)$, a bicephalus. Here the fact that $H\|\eta=J\| \eta$, and hence $\eta=\left(\rho^{+}\right)^{J}$, follows from condensation for $\omega$-sound mice (2.13). If $\rho$ is a non- $M$-cardinal (so $\gamma<\rho<\gamma^{+M}$ ), let $C=(\gamma, \rho, H, Q)$, where $Q \triangleleft M$ is least with $\rho \leq \mathrm{OR}^{Q}$ and $\rho_{\omega}^{Q}=\gamma$; here $C$ is a cephalanx, by 2.13 .

Claim 5. $C$ is a non-trivial, $\left(\omega_{1}+1\right)$-iterable cephal.

Assume this claim for now; we will use it to finish the proof.

Claim 6. Suppose that either:

(i) $\rho$ is a cardinal of $M$, so $C=(\rho, H, J)$ is a bicephalus; or

(ii) $\rho$ is not a cardinal of $M$ and $C=(\gamma, \rho, H, Q)$ is a passive cephalanx, $\mathrm{OR}^{J}=\eta$ and $J$ is type 3 .

Then (1) holds.

Proof. Note $N^{C}=J$ in case (ii). Using 4.3/4.5, and as $H \neq J$ and $J$ is sound, note $\mathrm{OR}^{J}=\eta, J$ is type $1 / 3$, and letting $F=F^{J}$ and $\kappa=\operatorname{cr}(F)$, we have $\kappa<\gamma$ (in case (i), $\gamma=\rho$; in case (ii), $J$ is type 3 ), the $\kappa$-core $N$ of $H$ is $\kappa$-sound, and

$$
N\left\|\left(\kappa^{+}\right)^{N}=H\right\|\left(\kappa^{+}\right)^{H}=M \|\left(\kappa^{+}\right)^{M}
$$

(so $F$ is weakly amenable to $N$ ) and $H=\operatorname{Ult}_{k}(N, F)$. It follows that $\rho_{k+1}^{M} \leq$ $\rho_{k+1}^{H} \leq \rho_{k+1}^{N} \leq \kappa<\gamma$, so $H \notin M$ and $\pi$ is a $k$-embedding. Now $\zeta_{k+1}^{N} \leq \kappa$ since $N$ is $\kappa$-sound. But then by line (8) and as $\pi, i_{F}^{N, k}$ preserve generalized solidity witnesses, we get $i_{F}^{N, k}\left(z_{k+1}^{N}\right)=z_{k+1}^{H}$ and $z_{k+1}^{M}=\pi\left(z_{k+1}^{H}\right)$ and $\zeta_{k+1}^{M}=\zeta_{k+1}^{H}=$ $\zeta_{k+1}^{N}$, giving (a). Since $\rho_{k+1}^{H} \leq \zeta_{k+1}^{H}<\gamma$ we have (c), and (d) is trivial. 
Claim 7. Suppose $\rho$ is a non- $M$-cardinal, $C=(\gamma, \rho, H, Q)$ is a passive cephalanx (so $N^{C}=J$ ), and if $\mathrm{OR}^{J}=\eta$ then $J$ is non-type 3 . Then (2)(c) holds.

Proof. Using 4.5, $\mathrm{OR}^{J}=\eta, J$ is type $1, \rho_{k+1}^{Q}=\operatorname{cr}\left(F^{J}\right)=\gamma<\rho_{k}^{Q}$ and $H=$ $\operatorname{Ult}_{k}\left(Q, F^{J}\right)$, and since $J \unlhd Q$, therefore $\rho_{k+1}^{H}=\rho_{k+1}^{Q}=\gamma<\rho$.

Claim 8. Suppose $\rho$ is a non- $M$-cardinal and $C$ is an active cephalanx. Then either (1) or (2)(d) holds.

Proof. We have $C=(\gamma, \rho, H, Q)$ where $Q=M \mid \rho$ is active. Let $F=F^{Q}$. Apply 4.17 to $C$. If $C$ is non-exceptional then $C$ has a good core, and as before either $\rho_{k+1}^{H}<\gamma$ and (1) holds, or $\rho_{k+1}^{H}=\gamma$ and (2)(d) holds.

Now suppose that $C$ is exceptional, so $C$ has an exceptional core. Let

$$
K=\operatorname{cHull}_{k+1}^{H}\left(X \cup z_{k+1}^{H} \cup \vec{p}_{k}^{H}\right),
$$

where $X$ is defined as in 4.13. Let $\kappa=\operatorname{cr}(F)$. By 4.14, $K$ is $\kappa^{+K}$-sound, and $\rho_{k+1}^{K} \leq \kappa^{+K}$. Since $\kappa^{++K}=\kappa^{++M}$, therefore $K \notin M$. Since $Q \in M$ and

$\operatorname{Th}_{\mathrm{r} \Sigma_{k+1}}^{K}\left(\vec{p}_{k+1}^{K} \cup \kappa^{+K}\right)$ can be computed from $F^{Q}$ and $\operatorname{Th}_{\mathrm{r} \Sigma_{k+1}}^{H}\left(\vec{p}_{k+1}^{H} \cup \rho\right)$,

it follows that $H \notin M$, so $\pi$ is a $k$-embedding, as is $i_{F}^{K, k}$. So we must verify (1).

SUBCLAIM 8.1. If $\rho_{k+1}^{K}=\kappa^{+K}$ then (1) holds.

Proof. The argument here is similar to that used to illustrate the failure of solidity for long extender premice. By 4.14 , we have $\rho_{k+1}^{H}=\rho$ and $i_{F}^{K, k}\left(p_{k+1}^{K}\right)=$ $p_{k+1}^{H}$ and both $K, H$ are $(k+1)$-sound. Moreover,

$$
p_{k+1}^{M} \leq \pi\left(p_{k+1}^{H}\right)^{\wedge}\langle\rho\rangle
$$

because $K \notin M$ and by the calcuation above. Since $H$ is $(k+1)$-solid, therefore $p_{k+1}^{M} \backslash \rho=\pi\left(p_{k+1}^{H}\right)$. But for $\alpha \leq \rho$,

$$
\alpha<\rho \Longleftrightarrow \operatorname{Th}_{\mathrm{r} \Sigma_{k+1}}^{M}\left(\pi\left(\vec{p}_{k+1}^{H}\right) \cup \alpha\right) \in M,
$$

because (in the case that $\alpha=\rho) H \notin M$, and (in the case that $\alpha<\rho) \operatorname{cr}(\pi)=$ $\rho=\rho_{k+1}^{H}$. But line (9) gives $p_{k+1}^{M}=\pi\left(p_{k+1}^{H}\right)^{\curlywedge}\langle\rho\rangle$.

Now $z_{k+1}^{H}=p_{k+1}^{H}$ and $\zeta_{k+1}^{H}=\rho$, and (1)(a),(c), (d) follow.

Note that in the above case, $M$ is not $(k+1)$-solid.

SubClaim 8.2. If $\rho_{k+1}^{K} \leq \kappa<\zeta_{k+1}^{K}$ then (1) holds.

Proof. Suppose $\rho_{k+1}^{K} \leq \kappa$. Then $\zeta_{k+1}^{K}<\kappa^{+K}$, as otherwise,

$$
\operatorname{Th}_{\mathrm{r} \Sigma_{k+1}}^{K}\left(\rho_{k+1}^{K} \cup \vec{p}_{k+1}^{K}\right) \in K,
$$


impossible. So $\kappa<\zeta_{k+1}^{K}<\kappa^{+K}$, so $z_{k+1}^{H}=i_{F}^{K}\left(z_{k+1}^{K}\right)$ and $\zeta_{k+1}^{H}=\sup i_{F}^{K}$ " $\zeta_{k+1}^{K}$, by [12, 2.20]. So $\gamma<\zeta_{k+1}^{H}<\rho$. So to verify (a), it suffices to see

$$
\mathrm{Th}_{\mathrm{r} \Sigma_{k+1}}^{M}\left(\pi\left(z_{k+1}^{H}\right) \cup \zeta_{k+1}^{H} \cup \vec{p}_{k}^{M}\right) \notin M,
$$

so suppose otherwise. Then because $F^{Q} \in M$, we get

$$
\operatorname{Th}_{\mathrm{r} \Sigma_{k+1}}^{K}\left(\zeta_{k+1}^{K} \cup z_{k+1}^{K} \cup \vec{p}_{k}^{K}\right) \in M .
$$

But $\mathcal{P}(\kappa)^{K}=\mathcal{P}(\kappa)^{M}$, so the above theory is in $K$, a contradiction.

We also have $\rho_{k+1}^{H} \leq \rho_{k+1}^{K} \leq \kappa$, so (c) holds and (d) is trivial.

SubCLAIM 8.3. If $\zeta_{k+1}^{K} \leq \kappa$ then (1) holds.

Proof. This follows as before since $\mathcal{P}(\kappa)^{K}=\mathcal{P}(\kappa)^{H}=\mathcal{P}(\kappa)^{M}$.

This completes the proof of the claim.

Sketch of Proof of Claim 5. The basic approach is to lift iteration trees on $C$ to iteration trees on $M$. There are some details here that one must be careful with. For illustration, we assume that $C=(\gamma, \rho, H, M \mid \rho)$ is an active cephalanx. The other cases are similar (the bicephalus case a little different, but simpler). Recall that we have already reduced to the case that $\pi$ is c-preserving. In order to keep focus on the main points, we assume that $\pi$ is in fact $\mathrm{c}-\nu$-preserving (see [9]). This will allow us to inductively maintain that all lifting maps we encounter are c- $\iota$-preserving, keeping the copying process smooth. (If instead, $\pi$ is not $\nu$-preserving, one should just combine the copying process to follow with that given in [9]. In the next section we do provide details of a copying process, with resurrection, which incorporates those extra details.)

For a tree $\mathcal{T}$ on $C$ and $\alpha+1<\operatorname{lh}(\mathcal{T})$, we say $\mathcal{T}$ lift-drops at $\alpha+1$ iff $\alpha+1 \in \mathscr{Q}^{\mathcal{T}}$, $\operatorname{pred}^{\mathcal{T}}(\alpha+1) \in \mathscr{B}^{\mathcal{T}}$ and $[0, \alpha+1]_{\mathcal{T}}$ does not drop in model.

If $\mathcal{T}$ lift-drops at $\alpha+1$ then $Q$ is type 2 , and letting $\beta=\operatorname{pred}^{\mathcal{T}}(\alpha+1)$, we have $E_{\beta}^{\mathcal{T}}=F\left(Q_{\beta}^{\mathcal{T}}\right)$ and $\operatorname{cr}\left(j_{\beta, \alpha+1}^{\mathcal{T}}\right)=\operatorname{lgcd}\left(Q_{\beta}^{\mathcal{T}}\right)$.

Let $\Sigma$ be a $\left(k, \omega_{1}+1\right)$-iteration strategy for $M$. Consider building an iteration tree $\mathcal{T}$ on $C$, and lifting this to a $k$-maximal tree $\mathcal{U}$ on $M$, via $\Sigma$, inductively on $\operatorname{lh}(\mathcal{T})$. Having defined $(\mathcal{T}, \mathcal{U})\left\lceil\lambda+1\right.$, then for each $\alpha \leq \lambda$, letting $B_{\alpha}, M_{\alpha}, Q_{\alpha}$ be the models of $\mathcal{T}$, and $S_{\alpha}=M_{\alpha}^{\mathcal{U}}$, and $W_{\alpha}=i_{0 \alpha}^{\mathcal{U}}(Q)$ when $[0, \alpha]_{\mathcal{U}} \cap \mathscr{D}^{\mathcal{U}}=\emptyset$, we will have also defined embeddings $\pi_{\alpha}$ and $\sigma_{\alpha}$, such that:

1. $<_{\mathcal{T}} \uparrow(\lambda+1)=<_{\mathcal{U}} \uparrow(\lambda+1)$. The drop structure of $\mathcal{U}$ matches that of $\mathcal{T}$, except for the following exceptions:

- If $\alpha \in \mathscr{B}^{\mathcal{T}}$ then $[0, \alpha]_{\mathcal{U}}$ does not drop in model or degree (so $\operatorname{deg}^{\mathcal{U}}(\alpha)=$ $k)$.

- If $\mathcal{T}$ lift-drops at $\alpha$ then $\mathcal{U}$ drops in model at $\alpha$.

Moreover, if $\alpha \notin \mathscr{B}^{\mathcal{T}}$ then $\operatorname{deg}^{\mathcal{U}}(\alpha) \geq \operatorname{deg}^{\mathcal{T}}(\alpha)$.

2. Suppose $\alpha \in \mathscr{B}^{\mathcal{T}}$. Then: 
- $\pi_{\alpha}: \mathfrak{C}_{0}\left(M_{\alpha}\right) \rightarrow \mathfrak{C}_{0}\left(S_{\alpha}\right)$ is c- $\iota$-preserving $k$-lifting, and

- $\sigma_{\alpha}: Q_{\alpha} \rightarrow W_{\alpha}$ is an $\Sigma_{0}$-elementary simple embedding.

Moreover, $\sigma_{\alpha} \subseteq \pi_{\alpha}$.

3. Suppose $\alpha \in \mathscr{M}^{\mathcal{T}}$. Then:

- $\pi_{\alpha}: \mathfrak{C}_{0}\left(M_{\alpha}\right) \rightarrow \mathfrak{C}_{0}\left(S_{\alpha}\right)$ is c-ı-preserving $\operatorname{deg}^{\mathcal{T}}(\alpha)$-lifting, and

$-\sigma_{\alpha}$ is undefined.

4. Suppose $\alpha \in \mathscr{Q}^{\mathcal{T}}$. Then:

- $\pi_{\alpha}$ is undefined, and

- $\sigma_{\alpha}: \mathfrak{C}_{0}\left(Q_{\alpha}\right) \rightarrow \mathfrak{C}_{0}\left(S_{\alpha}\right)$ is c- $\iota$-preserving $\operatorname{deg}^{\mathcal{T}}(\alpha)$-lifting.

5. Suppose $\alpha<\lambda$. Let $\beta \in(\alpha, \lambda]$. If $E_{\alpha}^{\mathcal{T}} \in \mathbb{E}_{+}\left(M_{\alpha}^{\mathcal{T}}\right)$ let $\psi_{\alpha}=\psi_{\pi_{\alpha}}$; otherwise let $\psi_{\alpha}=\psi_{\sigma_{\alpha}}$. Let $\tau \in\left\{\pi_{\beta}, \sigma_{\beta}\right\}$. Then

$$
\psi_{\alpha}\left\lceil\mathrm{h}_{\alpha}^{\mathcal{T}} \subseteq \tau \text { and } \tau\left(\iota_{\alpha}^{\mathcal{T}}\right)=\psi_{\alpha}\left(\iota_{\alpha}^{\mathcal{T}}\right)=\nu_{\alpha}^{\mathcal{U}} .\right.
$$

6. Suppose $\alpha<\lambda$ and let $\delta=\operatorname{pred}^{\mathcal{T}}(\alpha+1)=\operatorname{pred}^{\mathcal{U}}(\alpha+1)$.

(a) Suppose $\mathcal{T}$ drops in model at $\alpha+1$. Then so does $\mathcal{U}$. If $\alpha+1 \in \mathscr{M}^{\mathcal{T}}$ then $\psi_{\delta}\left(M_{\alpha+1}^{* \mathcal{T}}\right)=S_{\alpha+1}^{* \mathcal{U}}$ and

$$
\pi_{\alpha+1} \circ i_{\alpha+1}^{* \mathcal{T}}=i_{\alpha+1}^{* \mathcal{U}} \circ \psi_{\delta}\left\lceil\mathfrak{C}_{0}\left(M_{\alpha+1}^{* \mathcal{T}}\right) .\right.
$$

If $\alpha+1 \in{Q^{\mathcal{T}}}^{\text {then }} \psi_{\delta}\left(Q_{\alpha+1}^{* \mathcal{T}}\right)=S_{\alpha+1}^{* \mathcal{U}}$ and

$$
\sigma_{\alpha+1} \circ j_{\alpha+1}^{* \mathcal{T}}=i_{\alpha+1}^{* \mathcal{U}} \circ \psi_{\delta}\left\lceil\mathfrak{C}_{0}\left(Q_{\alpha+1}^{* \mathcal{T}}\right) .\right.
$$

(b) Suppose $\mathcal{T}$ lift-drops at $\alpha+1$. Then $\mathcal{U}$ drops in model at $\alpha+1$ (but $[0, \delta]_{\mathcal{U}}$ does not drop in model or degree), $S_{\alpha+1}^{* \mathcal{U}}=i_{0 \delta}^{\mathcal{U}}(Q)=W_{\delta}$ and

$$
\sigma_{\alpha+1} \circ j_{\delta, \alpha+1}^{\mathcal{T}}=i_{\alpha+1}^{* \mathcal{U}} \circ \sigma_{\delta}
$$

7. If $\alpha<\lambda$ and $\alpha<\mathcal{T} \beta \leq \lambda$ and $(\alpha, \beta]_{\mathcal{T}}$ neither drops in model nor lift-drops, then:

- If $M_{\beta}$ is defined then $\pi_{\beta} \circ i_{\alpha, \beta}^{\mathcal{T}}=i_{\alpha, \beta}^{\mathcal{U}} \circ \pi_{\alpha}$.

- If $Q_{\beta}$ is defined then $\sigma_{\beta} \circ j_{\alpha, \beta}^{\mathcal{T}}=i_{\alpha, \beta}^{\mathcal{U}} \circ \sigma_{\alpha}$.

This completes the inductive hypotheses.

We now start the construction. We start with $\pi_{0}=\pi$ and $\sigma_{0}=$ id. Since $\operatorname{cr}\left(\pi_{0}\right)=\rho$, we have $\sigma_{0} \subseteq \pi_{0}$.

Now let $E_{\lambda}=E_{\lambda}^{\mathcal{T}}$ be given. We define $F_{\lambda}=E_{\lambda}^{\mathcal{U}}$ by copying in the usual manner. That is:

(i) Suppose $E_{\lambda} \in \mathbb{E}_{+}\left(M_{\lambda}\right)$. Then: 
- If $E_{\lambda}=F\left(M_{\lambda}\right)$ then $F_{\lambda}=F\left(S_{\lambda}\right)$.

- If $E_{\lambda} \neq F\left(M_{\lambda}\right)$ then $F_{\lambda}=\psi_{\pi_{\lambda}}\left(E_{\lambda}\right)$.

(ii) Suppose $E_{\lambda} \notin \mathbb{E}_{+}\left(M_{\lambda}\right)$; so $E_{\lambda} \in \mathbb{E}_{+}\left(Q_{\lambda}\right)$. Then:

- If $E_{\lambda}=F\left(Q_{\lambda}\right)$ and $[0, \lambda]_{\mathcal{T}}$ does not drop or lift-drop then $F_{\lambda}=$ $F\left(W_{\lambda}\right)$.

- If $E_{\lambda}=F\left(Q_{\lambda}\right)$ and $[0, \lambda]_{\mathcal{T}}$ drops or lift-drops then $F_{\lambda}=F\left(S_{\lambda}\right)$.

- If $E_{\lambda} \neq F\left(Q_{\lambda}\right)$ then $F_{\lambda}=\psi_{\sigma_{\lambda}}\left(E_{\lambda}\right)$.

The agreement hypotheses and the fact that $\pi_{\lambda}$ and $\sigma_{\lambda}$ are c- - -preserving (if defined) ensures that this choice of $F_{\lambda}$ is legitimate.

Let $\beta=\operatorname{pred}^{\mathcal{T}}(\lambda+1)$ and $\kappa=\operatorname{cr}_{\lambda}^{\mathcal{T}}$. We consider only the case that

$$
\beta \in \mathscr{B}^{\mathcal{T}} \text { and } \kappa \leq \gamma\left(B_{\beta}^{\mathcal{T}}\right) \text { and } \mathcal{T} \text { does not drop in model at } \lambda+1 \text {. }
$$

For otherwise it is routine to propagate the inductive hypotheses, except maybe for the $\iota$-preservation of the embeddings. But we give the details for $\iota$-preservation in the case we consider, and it is similar in general. So suppose line (10) holds. We have $\beta=\operatorname{pred}^{\mathcal{U}}(\lambda+1)$ by property 5 .

CASE I. $\lambda+1 \in \mathscr{B}^{\mathcal{T}}$.

In this case $[0, \lambda+1]_{\mathcal{U}}$ does not drop in model or degree; this is because $\pi_{\lambda}$ is cpreserving and because if $E_{\beta}=F\left(Q_{\beta}\right)$ then $\kappa<\gamma\left(B_{\beta}^{\mathcal{T}}\right)$. By 2.2 and properties 2 and 5 , we can apply (essentially) ${ }^{31}$ the Shift Lemma to $\left(\pi_{\beta}, \psi_{\lambda}\left\lceil\operatorname{ex}_{\lambda}^{\mathcal{T}}\right)\right.$ and $\left(\sigma_{\beta}, \psi_{\lambda}\left\lceil\mathrm{ex}_{\lambda}^{\mathcal{T}}\right)\right.$, to produce $\pi_{\lambda+1}$ and $\sigma_{\lambda+1}$. For the latter, we have

$$
\sigma_{\beta}: Q_{\beta} \rightarrow W_{\beta} \triangleleft S_{\beta}=S_{\lambda+1}^{*},
$$

and we set

$$
\sigma_{\lambda+1}\left([a, f]_{E_{\lambda}}^{Q_{\beta}}\right)=\left[\psi_{\lambda}(a), \sigma_{\beta}(f)\right]_{F_{\lambda}}^{S_{\beta}}
$$

It follows easily that $\sigma_{\lambda+1} \subseteq \pi_{\lambda+1}$.

Now $\iota$-preservation for $\sigma_{\lambda+1}$ is immediate because this embedding is simple. We verify that $\pi_{\lambda+1}$ is $\iota$-preserving. This is immediate unless $H$ is type 3 , so assume this. So $M_{\beta}, S_{\beta}, M_{\lambda+1}, S_{\lambda+1}$ are also type 3 , so $\iota$-preservation just means $\nu$-preservation here. Write $\nu^{M_{\beta}}=\nu\left(F^{M_{\beta}}\right)$ and $\nu^{S_{\beta}}$ likewise. Write $\psi_{\beta}=\psi_{\pi_{\beta}}$ and $\psi_{\lambda+1}$ likewise. Write $\psi_{\beta, \lambda+1}^{\mathcal{T}}=\psi_{i_{\beta, \lambda+1}^{\mathcal{T}}}$ and $\psi_{\beta, \lambda+1}^{\mathcal{U}}$ likewise. By induction, $\pi_{\beta}$ is $\nu$-preserving; that is, $\psi_{\beta}\left(\nu^{M_{\beta}}\right)=\nu^{S_{\beta}}$. We must see that $\pi_{\lambda+1}$ is also; that is, that

$$
\psi_{\lambda+1}\left(\nu^{M_{\lambda+1}}\right)=\nu^{S_{\lambda+1}} .
$$

But note that

$$
\psi_{\lambda+1} \circ \psi_{\beta, \lambda+1}^{\mathcal{T}}=\psi_{\beta, \lambda+1}^{\mathcal{U}} \circ \psi_{\beta}
$$

\footnotetext{
${ }^{31}$ We say essentially because if $Q$ is type $3, \sigma_{\beta}$ is a simple embedding, not an embedding between squashed premice.
} 
So if $i_{\beta, \lambda+1}^{\mathcal{T}}$ and $i_{\beta, \lambda+1}^{\mathcal{U}}$ are also $\nu$-preserving, then so is $\pi_{\lambda+1}$. This holds in particular if $k>0$, by elementarity considerations. So suppose $k=0$. Then

$$
M_{\lambda+1}=\operatorname{Ult}_{0}\left(M_{\beta}, E_{\lambda}\right),
$$

and note that

$$
\operatorname{Ult}\left(M_{\lambda+1}^{\mathrm{sq}}, F^{M_{\lambda+1}}\right)=\operatorname{Ult}\left(\operatorname{Ult}\left(M_{\beta}^{\mathrm{sq}}, F^{M_{\beta}}\right), E_{\lambda}\right),
$$

and the ultrapower maps commute, and a straightforward calculation with extenders show that

$$
\psi_{\beta, \lambda+1}^{\mathcal{T}}: \operatorname{Ult}\left(M_{\beta}^{\mathrm{sq}}, F^{M_{\beta}}\right) \rightarrow \operatorname{Ult}\left(M_{\lambda+1}^{\mathrm{sq}}, F^{M_{\lambda}+1}\right)
$$

(which, recall, is defined via the Shift Lemma) coincides with the resulting $E_{\lambda^{-}}$ ultrapower map. Likewise for $\psi_{\beta, \lambda+1}^{\mathcal{U}}$.

Now let $\mu=\operatorname{cof}^{M_{\beta}}\left(\nu^{M_{\beta}}\right)$. Then $\psi_{\beta}(\mu)=\operatorname{cof}^{S_{\beta}}\left(\psi_{\beta}\left(\nu^{M_{\beta}}\right)\right)=\operatorname{cof}^{S_{\beta}}\left(\nu^{S_{\beta}}\right)$.

By the preceding remarks, if $\kappa \neq \mu$ then $\psi_{\beta, \lambda+1}^{\mathcal{T}}$ and $\psi_{\beta, \lambda+1}^{\mathcal{U}}$ are continuous at $\nu^{M_{\beta}}$ and $\nu^{S_{\beta}}$ respectively, so by commutativity, $\pi_{\lambda+1}$ is $\nu$-preserving. So suppose $\kappa=\mu$, so $\pi_{\beta}(\kappa)=\operatorname{cof}^{S_{\beta}}\left(\nu^{S_{\beta}}\right)$. Let $f \in M_{\beta}$ with $f: \kappa \rightarrow \nu^{M_{\beta}}$ be cofinal. Write $f^{M_{\beta}}=f$. So

$$
f^{S_{\beta}}=\psi_{\beta}\left(f^{M_{\beta}}\right): \pi_{\beta}(\kappa) \rightarrow \nu^{S_{\beta}}
$$

is also cofinal. Let $f^{M_{\lambda+1}}=\psi_{\beta, \lambda+1}^{\mathcal{T}}\left(f^{M_{\beta}}\right)$ and $f^{S_{\lambda+1}}$ be likewise, so commutativity gives $\psi_{\lambda+1}\left(f^{M_{\lambda+1}}\right)=f^{S_{\lambda+1}}$. Note then that

$$
\nu^{M_{\lambda+1}}=\sup i_{\beta, \lambda+1} " \nu^{M_{\beta}}=\sup f^{M_{\lambda+1} \text { " } \kappa}
$$

and likewise

$$
\nu^{S_{\lambda+1}}=\sup i_{\beta, \lambda+1}^{\mathcal{U}} " \nu^{S_{\beta}}=\sup f^{S_{\lambda+1} \text { " } \pi_{\beta}(\kappa) .}
$$

Since $\psi_{\lambda+1}(\kappa)=\pi_{\beta}(\kappa)$, therefore

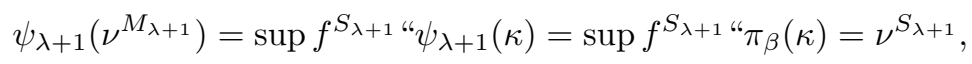

as desired.

The remaining properties for this case are established as usual.

CASE II. $\lambda+1 \in \mathscr{M}^{\mathcal{T}}$.

This case is routine, using the fact that $E_{\beta} \in \mathbb{E}_{+}\left(M_{\beta}\right)$.

CASE III. $\lambda+1 \in \mathscr{Q}^{\mathcal{T}}$.

So $\mathcal{T}$ lift-drops at $\lambda+1$, and so $E_{\beta}=F\left(Q_{\beta}\right)$ and $\mathrm{cr}_{\lambda}^{\mathcal{T}}=\gamma\left(B_{\beta}\right)$. Therefore $F_{\beta}=F\left(W_{\beta}\right)$ and $\operatorname{cr}_{\lambda}^{\mathcal{U}}=\sigma_{\beta}\left(\gamma\left(B_{\beta}\right)\right)$ is the largest cardinal of $W_{\beta}$. Therefore

$$
S_{\lambda+1}^{*}=W_{\beta} \triangleleft S_{\beta},
$$

and in particular, $\mathcal{U}$ drops in model at $\lambda+1$. This is precisely enough to define $\sigma_{\lambda+1}$. Everything else is routine in this case.

This completes the propagation of the properties to $(\mathcal{T}, \mathcal{U})\lceil\lambda+2$.

For limit $\lambda$, everything is routine.

This completes the sketch of the proof that $C$ is iterable, and so the proof of the theorem. 


\section{A premouse inner model inheriting strong cardinals}

Let $W \models$ ZFC be an iterable transitive model. In this section we define a proper class premouse $L[\mathbb{E}]^{W}$ of $W$ which inherits all Woodin and strong cardinals from $W$. (See $\S 1$ for some introduction to this and a comparison with Steel's local $K^{c}$-construction of [15].) The construction allows certain types of partial background extenders. However, all background extenders will be total in some ultrapower of $W$, and moreover, assuming enough AC, we will be able to lift iteration trees on $L[\mathbb{E}]^{W}$ to (non-dropping) iteration trees on $W$. The model $L[\mathbb{E}]^{W}$ is also outright definable over $W$.

Let us first point out that a fully backgrounded construction can fail to inherit strong cardinals:

6.1 Remark. Assume ZFC and suppose $\kappa$ is strong but there is no measurable cardinal $\mu>\kappa$. Let $\left\langle N_{\alpha}\right\rangle_{\alpha \leq \mathrm{OR}}$ be a fully backgrounded $L[\mathbb{E}]$-construction; suppose that this does not break down, so produces a model $L[\mathbb{E}]=N_{\mathrm{OR}}$ of height OR. Then we claim that $\mathbb{E}$ has no extenders with index $\geq \kappa$, and hence $\kappa$ is certainly not strong in $L[\mathbb{E}]$. For let $\zeta \in \mathrm{OR}$ be such that $L[\mathbb{E}] \mid \kappa=N_{\zeta}$. Then we claim there is no $\alpha \geq \zeta$ such that $N_{\alpha}$ is active, which suffices. For suppose otherwise and let $\alpha$ be least such. Since $\mathrm{OR}^{N_{\zeta}}=\kappa$ is a cardinal, we have $\alpha>\zeta$. So $\alpha=\beta+1$ where $\beta=\mathrm{OR}^{N_{\beta}}>\kappa$ and $N_{\beta}=\mathcal{J}_{\beta}\left(N_{\zeta}\right)$. Let $\mu=\operatorname{cr}\left(F^{N_{\beta+1}}\right)$. Since the construction is fully backgrounded, $\mu$ is measurable, so $\mu \leq \kappa$. Since $N_{\beta}=\mathcal{J}_{\beta}\left(N_{\zeta}\right)$ and by coherence, it easily follows there is $\gamma<\mu$ such that $N_{\zeta}=\mathcal{J}_{\kappa}\left(N_{\zeta} \mid \gamma\right)$. But then note that $\rho_{\omega}\left(N_{\beta+1}\right) \leq \gamma<\mu \leq \kappa$, contradicting the fact that $N_{\zeta}=L[\mathbb{E}] \mid \kappa$.

Of course if the background construction does not make unusual demands on background extenders, then $L[\mathbb{E}] \mid \kappa$ is closed under \#'s. In this case, note that extenders $E$ in $V$ with $\operatorname{cr}(E)=\kappa$ do not cohere $L[\mathbb{E}]$.

Instead of using rank to measure the strength of extenders, we use:

6.2 Definition. Let $E$ be an extender. The strength of $E$, denoted $\operatorname{str}(E)$, is the largest $\rho$ such that $H_{\rho} \subseteq \operatorname{Ult}(V, E)$.

So $\operatorname{str}(E)$ is always a cardinal. The backgrounding we use is described as follows (in the definition, we imagine we are working inside $W$ as mentioned earlier):

6.3 Definition. Assume ZFC. Let $\lambda \leq \mathrm{OR}+1$. An ultra-backgrounded construction (of length $\lambda$ ) is a sequence $\left\langle S_{\alpha}\right\rangle_{\alpha<\lambda}$ such that:

1. Each $S_{\alpha}$ is a premouse.

2. Given a limit $\beta<\lambda, S_{\beta}=\liminf _{\alpha<\beta} S_{\alpha}$.

3. Given $\beta=\alpha+1<\lambda$, either:

(a) For each $n<\omega, \mathfrak{C}_{n}\left(S_{\alpha}\right)$ is $(n+1)$-universal and $\mathfrak{C}_{n+1}\left(S_{\alpha}\right)$ is $(n+1)$ solid, and $S_{\alpha+1}=\mathcal{J}\left(\mathfrak{C}_{\omega}\left(S_{\alpha}\right)\right)$; or 
(b) $S_{\alpha}$ is passive and there is $F$ and an extender $G$ such that $S_{\alpha+1}=$ $\left(S_{\alpha}, F\right)$ and $F\lceil\nu(F) \subseteq G$ and $\operatorname{str}(G) \geq \nu(F)$; or

(c) $\alpha$ is a limit, $S_{\alpha}$ has a largest cardinal $\rho$, and there is an extender $G$ such that letting $\kappa=\operatorname{cr}(G)$, we have:

$$
\begin{aligned}
& \text { i. } \operatorname{str}(G) \geq \rho, \\
& \text { ii. } \kappa \leq \rho \leq i_{G}(\kappa), \\
& \text { iii. } \rho \text { is a cardinal in } i_{G}\left(S_{\alpha}\right), \\
& \text { iv. }\left(S_{\alpha} \sim i_{G}\left(S_{\alpha}\right)\right) \| \operatorname{OR}\left(S_{\alpha}\right), \\
& \text { v. } S_{\alpha+1} \triangleleft i_{G}\left(S_{\alpha}\right), \\
& \text { vi. } \rho_{\omega}\left(S_{\alpha+1}\right)=\rho, \\
& \text { vii. } \operatorname{OR}\left(S_{\alpha}\right)=\left(\rho^{+}\right)^{S_{\alpha+1}} .
\end{aligned}
$$

6.4 Definition. Suppose that $V$ is a premouse (and ZFC holds). A pm-ultrabackgrounded construction is a sequence $\left\langle S_{\alpha}\right\rangle_{\alpha<\lambda}$ as in 6.3, except that in (3b) and (3c) we also require that $G \in \mathbb{E}^{V}$ and $\nu(G)$ is a cardinal.

6.5 Remark. When we refer to, for example, 6.4(3c), we mean the analogue of $6.3(3 \mathrm{c})$ for 6.4 . We will mostly work explicitly with ultra-backgrounded constructions; the adaptation to pm-ultra-backgrounded is mostly obvious, so we mostly omit it. For all definitions to follow, we either implicitly or explicitly make the pm-ultra-backgrounded analogue, denoted by the prefix pm-.

6.6 Definition. Let $\mathbb{C}=\left\langle S_{\alpha}\right\rangle_{\alpha<\lambda}$ be an ultra-backgrounded construction. Let $\beta<\lambda$. Then we say that $\beta$, or $S_{\beta}$, is $\mathbb{C}$-standard iff $6.3(2)$, (3a) or (3b) holds (for $\beta$ ). We say that $\beta$ is $\mathbb{C}$-strongly standard iff $6.3(3 \mathrm{c})$ does not hold. Given also $n \leq \omega$, we say that $(\beta, n)$ is $\mathbb{C}$-relevant iff either (i) $\beta$ is $\mathbb{C}$-standard, or (ii) $\beta=\alpha+1$ and $\rho_{n}\left(N_{\alpha+1}\right)=\rho_{\omega}\left(N_{\alpha+1}\right)$.

Clearly $\mathbb{C}$-strongly standard implies $\mathbb{C}$-standard. The next lemma is routine:

6.7 Lemma. Let $\mathbb{C}=\left\langle S_{\alpha}\right\rangle$ be an ultra-backgrounded construction. Let $(\beta, n)$ be $\mathbb{C}$-relevant. Let $\rho$ be a cardinal of $S_{\beta}$ such that $\rho \leq \rho_{n}^{S_{\beta}}$. Let $P \triangleleft S_{\beta}$ be such that $\rho_{\omega}^{P}=\rho$. Then there is $\alpha<\beta$ such that $\mathfrak{C}_{0}(P)=\mathfrak{C}_{\omega}\left(S_{\alpha}\right)$.

6.8 Remark. It follows that if $6.3(3 \mathrm{c})$ holds, there is $\xi$ such that $S_{\alpha+1}=$ $\mathfrak{C}_{\omega}\left(S_{\xi}^{i_{G}(\mathbb{C})}\right)$, because $\alpha$ is a limit and $\rho$ is a cardinal of $i_{G}\left(S_{\alpha}\right)$.

6.9 Lemma. Let $\mathbb{C}=\left\langle S_{\alpha}\right\rangle$ be an ultra-backgrounded construction. Suppose that $S_{\alpha+1}$ is active type 1 or type 3 and $\rho_{\omega}\left(S_{\alpha+1}\right)=\nu\left(F\left(S_{\alpha+1}\right)\right)$. Then $\alpha+1$ is $\mathbb{C}$-standard, so $F\left(S_{\alpha+1}\right)$ is backgrounded by a $V$-extender.

Proof. Suppose not and let $\alpha$ be the least counterexample. Let $\rho$ be the largest cardinal of $S_{\alpha}$. By $6.7, S_{\alpha} \mid \rho=S_{\beta}$ for some limit $\beta<\alpha$. Let $G$ be as in 6.3(3c) for $S_{\alpha+1}$. So $\operatorname{Ult}(V, G)$ satisfies "the lemma holds for $i_{G}^{V}(\mathbb{C}\lceil\beta)$ ", and note that $i_{G}^{V}(\rho)>\rho$, and $i_{G}^{V}\left(S_{\alpha}\right) \mid i_{G}^{V}(\rho)=i_{G}^{V}\left(S_{\beta}\right)$ and $\rho$ is a cardinal of $i_{G}^{V}\left(S_{\beta}\right)$. So by 6.7, $S_{\alpha+1}=\mathfrak{C}_{\omega}\left(S_{\gamma}^{i_{G}^{V}(\mathbb{C} \mid \beta)}\right)$ for some $\gamma$. But because $S_{\alpha+1}$ is type 1 or 3 and by the 
ISC, it follows that $S_{\gamma}^{i_{G}^{V}(\mathbb{C} \mid \beta)}$ is already fully sound, so $S_{\alpha+1}=S_{\gamma}^{i_{G}^{V}(\mathbb{C} \mid \beta)}$. But then since $\operatorname{Ult}(V, G)$ thinks the lemma holds for $i_{G}^{V}(\mathbb{C}\lceil\beta)$, therefore $\operatorname{Ult}(V, G) \models$ " $\gamma$ is $i_{G}^{V}(\mathbb{C} \uparrow \beta)$-standard". So there is $H \in \operatorname{Ult}(V, G)$ such that in $\operatorname{Ult}(V, G) \models$ " $H$ is a $\rho$-strong extender" and $F\left\lceil\nu \subseteq H\right.$, where $F=F^{S_{\alpha+1}}$ and $\nu=\nu(F)$. But since $G$ is $\rho$-strong, so is $H$ (in $V$ ), and since $\nu \leq \rho$ (as $F$ is type 1 or 3 ), $H$ backgrounds $F$ in $V$, so $\alpha+1$ is $\mathbb{C}$-standard, a contradiction.

6.10 Definition. Let $\mathbb{C}=\left\langle S_{\alpha}\right\rangle$ be an ultra-backgrounded construction. Suppose that $\alpha+1$ is not $\mathbb{C}$-standard, and let $\rho=\rho_{\omega}\left(S_{\alpha+1}\right)$. An extender $G$ is a $\mathbb{C}$-nice witness for $\alpha+1$ iff $G$ witnesses $6.3(3 \mathrm{c}), i_{G}(\operatorname{cr}(G))>\rho$, and $S_{\alpha+1}$ is $i_{G}(\mathbb{C})$-strongly standard (in $\operatorname{Ult}(V, G)$ ).

6.11 Lemma. Let $\mathbb{C}=\left\langle S_{\alpha}\right\rangle$ be an ultra-backgrounded construction. Suppose that $\alpha+1$ is not $\mathbb{C}$-standard and let $\rho=\rho_{\omega}\left(S_{\alpha+1}\right)$. Then there is a $\mathbb{C}$-nice witness for $\alpha+1$.

Let $G$ be a $\mathbb{C}$-nice witness for $\alpha+1$. Then:

- If $\operatorname{cr}(G)<\rho$ then $\operatorname{str}(G)$ is the the least cardinal $\geq \rho$.

- If $\operatorname{cr}(G)=\rho$ then $\operatorname{str}(G)=\rho^{+}$.

- If condensation for $\omega$-sound mice holds for all proper segments of $S_{\alpha}$ then $\rho$ is not measurable in $\operatorname{Ult}(V, G)$.

Proof. Because $V$ is linearly iterable and $\alpha+1$ is not $\mathbb{C}$-standard, there is an extender $H$ witnessing $6.3(3 \mathrm{c})$ and such that $\operatorname{Ult}(V, H) \models " \xi$ is $i_{H}(\mathbb{C})$-strongly standard", where $\xi$ is defined as in 6.8. Letting $G=i_{H}(H) \circ H$, then $G$ is a nice witness $\left(S_{\alpha+1} \triangleleft i_{G}\left(S_{\alpha}\right)\right.$ because in $\operatorname{Ult}(V, H), i_{H}(H)$ coheres $i_{H}\left(S_{\alpha}\right)$ enough).

Now let $G$ be a nice witness. The facts regarding $\operatorname{str}(G)$ are easy. Suppose $F$ is a measure on $\rho$ in $U=\operatorname{Ult}(V, G)$. Then by condensation, $S_{\alpha+1} \triangleleft i_{F}^{U}\left(S_{\alpha+1}\right)$, contradicting the niceness of $G$.

For pm-ultra-backgrounding, we need to modify the notion of nice witness a little:

6.12 Definition. Suppose $V$ is a premouse and let $\mathbb{C}=\left\langle S_{\alpha}\right\rangle$ be a pm-ultrabackgrounded construction. Suppose that $\alpha+1$ is not pm- $\mathbb{C}$-standard, and let $\rho=\rho_{\omega}\left(S_{\alpha+1}\right)$. The pm- $\mathbb{C}$-nice witness for $\alpha+1$ is the extender $G$ such that, letting $G_{1}$ be the least witness to $6.4(3 \mathrm{c})$ (that is, the witness with $\operatorname{lh}\left(G_{1}\right)$ minimal), either:

(i) $S_{\alpha+1}$ is pm- $i_{G_{1}}(\mathbb{C})$-strongly standard and $G=G_{1}$, or

(ii) $S_{\alpha+1}$ is not pm- $i_{G_{1}}(\mathbb{C})$-strongly standard and letting $G_{2}$ be the least witness to $6.4(3 \mathrm{c})$ for $\left(i_{G_{1}}(\mathbb{C}), S_{\alpha+1}\right)$, then $G=G_{2} \circ G_{1}$.

6.13 Lemma. Suppose $V$ is a premouse and let $\mathbb{C}=\left\langle S_{\alpha}\right\rangle$ be a pm-ultrabackgrounded construction. Suppose that $\alpha+1$ is not pm- $\mathbb{C}$-standard, let $\rho=$ $\rho_{\omega}\left(S_{\alpha+1}\right)$ and let $G$ be the pm- $\mathbb{C}$-nice witness for $\alpha+1$. Suppose that condensation for $\omega$-sound mice holds for all proper segments of $S_{\alpha}$. Then: 
- $S_{\alpha+1}$ is pm-i $i_{G}(\mathbb{C})$-strongly standard.

- $\rho$ is not measurable in $\operatorname{Ult}(V, G)$, so $i_{G}(\operatorname{cr}(G))>\rho$.

- If 6.12(i) attains and $\rho$ is not a cardinal then $\nu(G)=\rho^{+}$.

- If 6.12(i) attains $\rho$ is a cardinal then either $\nu(G)=\rho$, or $G$ is type 1 and $\operatorname{cr}(G)=\rho$.

- If 6.12(ii) attains then $\rho$ is a cardinal and letting $G_{1}, G_{2}$ be as there, $\nu\left(G_{1}\right)=\operatorname{cr}\left(G_{2}\right)=\rho$ and $G_{2}$ is type 1 .

Proof. By coherence and the ISC, and using condensation as in 6.11.

We now introduce what is, at least assuming global choice, a natural maximal ultra-backgrounded construction:

6.14 Definition. The ultra-stack construction is the sequence $\left\langle R_{\alpha}\right\rangle_{\alpha \leq \mathrm{OR}}$ such that $R_{0}=V_{\omega}$, the sequence is continuous at limits, and for each $\alpha<$ OR we have the following. Let $\rho=\operatorname{OR}\left(R_{\alpha}\right)$. Then $R_{\alpha+1}$ is the stack of all sound premice $R$ such that $R_{\alpha} \triangleleft R$ and $\rho_{\omega}^{R}=\rho$ and $R=\mathfrak{C}_{\omega}\left(S_{\gamma}^{\mathbb{C}}\right)$ for some ultra-backgrounded construction $\mathbb{C}$ and $\gamma<\operatorname{lh}(\mathbb{C})$, assuming this stack forms a premouse (if it does not, the construction not well-defined).

In order to prove that the ultra-stack construction inherits strong and Woodin cardinals, we will need to prove that certain pseudo-premice are in fact premice, just like in [3]. So we make one further definition:

6.15 Definition. Let $\lambda<$ OR. An ultra-backgrounded pseudo-construction (of length $\lambda+2$ ) is a sequence $\mathbb{C}=\left\langle S_{\alpha}\right\rangle_{\alpha<\lambda+2}$ such that:

- $\mathbb{C}\left\lceil\lambda+1\right.$ is an ultra-backgrounded construction and $S_{\lambda}$ is passive,

- For some $F, S_{\lambda+1}=\left(S_{\lambda}, F\right)$ is an active pseudo-premouse, and there is an extender $G$ such that $F\lceil\nu(F) \subseteq G$ and $\operatorname{str}(G) \geq \nu(F)$.

6.16 Definition. An almost normal iteration tree $\mathcal{U}$ on a premouse $P$ is an iteration tree as defined in [1], ${ }^{32}$ such that for all $\alpha+1<\beta+1<\operatorname{lh}(\mathcal{U})$, we have $\nu\left(E_{\alpha}^{\mathcal{T}}\right) \leq \nu\left(E_{\beta}^{\mathcal{T}}\right)$.

6.17 Remark. It is easy to see that if $P$ is a normally iterable premouse then $P$ is iterable with regard to almost normal trees.

We can now state the main theorem of this section:

6.18 Theorem. Assume ZF. Let $W \models Z F C$ be a transitive class, and suppose there is an $\left(\omega_{1}+1\right)$-iteration strategy for $W$ for arbitrary coarse trees. Then:

\footnotetext{
${ }^{32}$ The only difference between these and normal trees is that it is not required that $\operatorname{lh}\left(E_{\alpha}^{\mathcal{T}}\right)<$ $\operatorname{lh}\left(E_{\beta}^{\mathcal{T}}\right)$ for $\alpha<\beta$.
} 
(a) If $\lambda \in \mathrm{OR}^{W}$ and $\mathbb{C}=\left\langle S_{\alpha}\right\rangle_{\alpha \leq \lambda} \in W \models$ "C is an ultra-backgrounded construction" and $n<\omega$, then $\mathfrak{C}_{n}\left(S_{\alpha}\right)$ exists and is $\left(n, \omega_{1}, \omega_{1}+1\right)^{*}$-iterable, and therefore $\mathfrak{C}_{n}\left(S_{\alpha}\right)$ is $(n+1)$-universal and $\mathfrak{C}_{n+1}\left(S_{\alpha}\right)$ is $(n+1)$-solid.

(b) The ultra-stack construction of $W$ is well-defined. Let $L[\mathbb{E}]$ be its final model.

(c) If there is a class wellorder $<^{W}$ of $W$ then $L[\mathbb{E}]$ is $\left(0, \omega_{1}, \omega_{1}+1\right)^{*}$-iterable. ${ }^{33}$

(d) $W \models$ " $\kappa$ is strong" iff $L[\mathbb{E}] \models " \kappa$ is strong".

(e) If $W \models$ " $\delta$ is Woodin" then $L[\mathbb{E}] \models$ " $\delta$ is Woodin".

6.19 Theorem. Let $W \models \mathrm{ZFC}$ be a premouse (possibly proper class) which is $\left(\omega, \omega_{1}, \omega_{1}+1\right)^{*}$-iterable. Then the conclusions of 6.18 hold, with ultra replaced by pm-ultra.

6.20 Remark. Part (d) also holds for $A$-strong cardinals $\kappa$, for $A \subseteq \mathrm{OR}$ such that $A$ is a class of $L[\mathbb{E}]$. (Here $\kappa$ is $A$-strong iff for every $\eta$ there is an $\eta$-strong extender $G$ such that $i_{G}(A) \cap \eta=A \cap \eta$.)

However, (d) does not seem to hold for local strength: it seems that we might have $\kappa$ being $\eta$-strong (some $\eta \in \mathrm{OR}$ ) but $L[\mathbb{E}] \models$ " $\kappa$ is not $\eta$-strong".

Proof. Each part will depend on the sufficient iterability of certain structures, which we will establish in Claim 5 below. We first reduce everything to that iterability. We write $\left\langle R_{\eta}\right\rangle_{\eta \in \mathrm{OR}}{ }^{W}$ for the ultra-stack construction of $W$.

Claim 1. Work in $W$. Let $\eta \in$ OR. Then:

(i) $R_{\eta}$ is well-defined.

(ii) There is an ultra-backgrounded construction $\mathbb{C}=\left\langle S_{\alpha}\right\rangle_{\alpha \leq \lambda}$ with $S_{\lambda}=R_{\eta}$.

(iii) Let $\mathbb{C}=\left\langle S_{\alpha}\right\rangle_{\alpha \leq \lambda}$ be an ultra-backgrounded construction such that $R_{\eta}=$ $S_{\beta}$ for some $\beta \leq \lambda$. Then for all $\alpha \in[\beta, \lambda]$, we have $R_{\eta}=S_{\alpha} \mid \rho$ and $\rho_{\omega}\left(S_{\alpha}\right) \geq \rho$, and if $\beta<\alpha$ then $\rho$ is a cardinal of $S_{\alpha}$.

(iv) Let $\mathbb{C}=\left\langle S_{\alpha}\right\rangle_{\alpha \leq \lambda}$ and $\mathbb{C}^{\prime}=\left\langle S_{\alpha}^{\prime}\right\rangle_{\alpha \leq \lambda^{\prime}}$ be ultra-backgrounded constructions such that $R_{\eta}=S_{\beta}=S_{\beta^{\prime}}^{\prime}$ for some $\beta \leq \lambda$ and $\beta^{\prime} \leq \lambda^{\prime}$. Suppose $\rho_{\omega}\left(S_{\lambda}\right)=$ $\rho$. Suppose there is $\xi<\lambda^{\prime}$ such that $\mathfrak{C}_{\omega}\left(S_{\xi}^{\prime}\right)=\mathfrak{C}_{\omega}\left(S_{\lambda}\right)$. Then

$$
\mathbb{C}^{\wedge}\left(\mathbb{C}^{\prime} \uparrow\left(\xi^{\prime}, \lambda^{\prime}\right]\right)
$$

is also an ultra-backgrounded construction.

\footnotetext{
${ }^{33}$ The class wellorder $<^{W}$ need not be a class of $W$. It is only used to allow us to select background extenders canonically when copying iteration trees to $W$.
} 
Proof. The proof is by induction on $\eta$. When $\eta=0$ it is easy.

Suppose $\eta$ is a limit. Clearly $R_{\eta}$ is well-defined, giving part (i). Part (ii): Let $\left\langle\rho_{\alpha}\right\rangle_{\xi<\gamma}$ enumerate the infinite cardinals of $R_{\eta}$. Note that by induction, $\eta=\gamma$ and $R_{\xi}=R_{\eta} \mid \rho_{\xi}$ and there is an ultra-backgrounded construction $\mathbb{C}_{\xi}=$ $\left\langle S_{\xi \alpha}\right\rangle_{\alpha \leq \lambda_{\xi}}$ with $R_{\xi}=S_{\xi \lambda_{\xi}}$. Also by induction (applying part (iv)), we can merge these constructions into a single ultra-backgrounded construction $\mathbb{C}$ with last model $R_{\eta}$. That is, we set

$$
\mathbb{C}=\left(\mathbb { C } _ { 0 } \lceil [ 0 , \lambda _ { 0 } ) ) ^ { \wedge } \left(\mathbb{C}_{1}\left\lceil\left(\lambda_{0}^{\prime}, \lambda_{1}\right]\right)^{\curlywedge} \ldots,\right.\right.
$$

where $S_{1 \lambda_{0}^{\prime}}=S_{0 \lambda_{0}}=R_{\eta} \mid \aleph_{1}^{R_{\eta}}$, etc.

For the next two parts, the proof is identical in the limit and successor cases:

Part (iii): Suppose otherwise and let $\mathbb{C}=\left\langle S_{\alpha}\right\rangle_{\alpha \leq \lambda}$ be a counterexample of minimal length. Let $k<\omega$ be such that $\mathfrak{C}_{k}\left(S_{\alpha}\right)$ exists and $\rho_{k+1}^{S_{\alpha}}<\rho$. In Claim 5 we will show that

$$
\mathfrak{C}_{k}\left(S_{\alpha}\right) \text { is }\left(k, \omega_{1}, \omega_{1}+1\right)^{*} \text {-iterable in (the background) } V \text {. }
$$

It follows (iterating this) that $\mathfrak{C}_{\omega}\left(S_{\alpha}\right)$ exists, hence is $\omega$-sound, and $\rho_{\omega}\left(S_{\alpha}\right)<\rho$. But then the existence of $\mathbb{C}$ contradicts the maximality of $S_{\eta}$ (with respect to mice projecting to $\rho_{\omega}\left(S_{\alpha}\right)$ ).

Part (iv): This follows easily from the definitions (noting that in 6.3, we do not require the $V$-extenders to cohere the construction $\mathbb{C}$ (i.e., the sequence of models); the only kind of coherence required is with respect to individual models $S_{\alpha}$ ).

Now suppose that $\eta=\xi+1$.

Part (i): Suppose not. Then it is easy to see that we have ultra-backgrounded constructions

$$
\mathbb{C}=\left\langle S_{\alpha}\right\rangle_{\alpha \leq \lambda^{\prime}} \wedge\left\langle S_{\alpha}^{\mathbb{C}}\right\rangle_{\lambda^{\prime}<\alpha \leq \lambda^{\mathbb{C}}}
$$

and

$$
\widetilde{\mathbb{C}}=\left\langle S_{\alpha}\right\rangle_{\alpha \leq \lambda^{\prime}} \hat{\sim}\left\langle S_{\alpha}^{\widetilde{\mathbb{C}}}\right\rangle_{\lambda^{\prime}<\alpha \leq \lambda^{\widetilde{\mathbb{C}}}}
$$

and $\rho \in \mathrm{OR}$ such that letting $M^{\prime}=S_{\lambda^{\mathbb{C}}}^{\mathbb{C}}$ and $N^{\prime}=S_{\lambda^{\widetilde{\mathbb{C}}}}^{\widetilde{\mathbb{C}}}$ :

- $M=\mathfrak{C}_{\omega}\left(M^{\prime}\right)$ and $N=\mathfrak{C}_{\omega}\left(N^{\prime}\right)$ both exist,

- $\rho_{\omega}^{M}=\rho=\rho_{\omega}^{N}$,

- $S_{\lambda^{\prime}}=M\left\|\rho^{+M}=N\right\| \rho^{+N}=M^{\prime}\left\|\rho^{+M^{\prime}}=N^{\prime}\right\| \rho^{+N^{\prime}}$, but

$-M \neq N$.

It follows that $C=(\rho, M, N)$ is a sound, non-trivial bicephalus. In Claim 5 below, we will show that

$$
C \text { is }\left(\omega_{1}+1\right) \text {-iterable in (the background) } V,
$$

contradicting 4.3 .

Part (ii): This is much as in the limit case, but by merging constructions which end in mice projecting to $\rho$. 
It easily follows (from Claim 5) that:

Claim 2. Work in $W$. Then $L[\mathbb{E}]=R_{\mathrm{OR}}$ is well-defined, and the cardinal segments of $L[\mathbb{E}]$ are exactly the models $R_{\alpha}$ for $\alpha \in$ OR.

So we have reduced (b) to Claim 5 . We next reduce (d) and (e). The fact that every strong cardinal of $L[\mathbb{E}]$ is strong in $W$ is by 6.9. So suppose that either $\kappa$ is strong in $W$ or $\delta$ is Woodin in $W$; we want to see that $\kappa$ is strong in $L[\mathbb{E}]$ or $\delta$ Woodin in $L[\mathbb{E}]$ respectively. The key to the strong case is the following claim.

Claim 3. Work in $W$. Let $\tau$ be a cardinal of $L[\mathbb{E}]$ and $R_{\alpha}$ be such that $\tau=$ $\mathrm{OR}^{R_{\alpha}}$. Then there is $\chi>\tau$ such that if $F$ is any extender with arbitrary critical point and $\operatorname{str}(F) \geq \chi$ then $i_{F}\left(R_{\alpha}\right) \mid \rho=R_{\alpha}$.

Proof. Let $\chi$ be such that there is an ultra-backgrounded construction $\mathbb{C} \in \mathcal{H}_{\chi}$ with last model $R_{\alpha}$ (using Claim 1), and such that $\mathcal{H}_{\chi}$ includes background extenders witnessing the clauses of 6.3 for $\mathbb{C}$. It is straightforward to see that $\chi$ works.

6.21 Remark. Note that Claim 3 can fail for fully backgrounded $L[\mathbb{E}]$-constructions, by the last paragraph of 6.1. The key difference is that any mice projecting $<\tau$ which are added by $i_{F}(\mathbb{C})$ (when $F$ is strong enough) are, by definition, added to the ultra-stack construction; this, however, is not true of fully backgrounded constructions (the extenders used in the construction of these projecting mice might be total in $\operatorname{Ult}(V, F)$, but partial in $V)$.

Using the claim, together with a slight variant of the proof of [3, Lemma $11.4]$, one can show that strength and Woodinness in $W$ is absorbed by $L[\mathbb{E}]$, as witnessed by restrictions of extenders in $W$. The details of the argument relating to the uniqueness of the next extender are somewhat different, so we describe the differences. We will not reproduce all the details or definitions from that text, so the reader should have it in hand.

Let $\tau$ be a cardinal of $L[\mathbb{E}]$, and $F \in W$ be a $W$-extender with

$$
\operatorname{cr}(F)<\tau \leq i_{F}(\operatorname{cr}(F)) \text { and } i_{F}(L[\mathbb{E}])|\tau=L[\mathbb{E}]| \tau .
$$

We get these as usual from Woodinness, and by the preceding claim, we also get them with $\operatorname{cr}(F)=\kappa$ if $\kappa<\tau$ and $\kappa$ is strong in $W$. We adopt now the notation " $\rho$ " and " $G$ " of [3, Lemma 11.4].

Claim 4. [3, Lemma 11.4] holds for all $\rho<\tau$ such that $G$ is not type Z.

Therefore, if $W \models$ " $\kappa$ is strong" then $L[\mathbb{E}] \models$ " $\kappa$ is strong", and if $W \models$ " $\delta$ is Woodin" then $L[\mathbb{E}] \models$ " $\delta$ is Woodin", and these facts are witnessed by restrictions of extenders in $W$.

Proof. Recall that the proof is by induction on $\rho$. Let

$$
\sigma: \operatorname{Ult}(L[\mathbb{E}], G) \rightarrow \operatorname{Ult}(L[\mathbb{E}], F)
$$

be the natural factor map. Let $\xi=\left(\rho^{+}\right)^{\mathrm{Ult}(L[\mathbb{E}], G)}$. By Claim 5, condensation holds for segments of $L[\mathbb{E}]$, and so because of the existence of $\sigma$, either: 
(i) $L[\mathbb{E}] \mid \rho$ is passive and $\operatorname{Ult}(L[\mathbb{E}], G)\|\xi=L[\mathbb{E}]\| \xi$, or

(ii) $L[\mathbb{E}] \mid \rho$ is active and $\operatorname{Ult}(L[\mathbb{E}], G)\left\|\xi=\operatorname{Ult}\left(L[\mathbb{E}], F^{L[\mathbb{E}] \mid \rho}\right)\right\| \xi$.

Suppose first that $\rho$ is a cardinal of $L[\mathbb{E}]$, and so (i) holds. Then there is an ultra-backgrounded construction with last model $P=(L[\mathbb{E}] \| \xi, G)$. It follows that $\rho_{\omega}^{P}=\rho$, so $P$ is fully sound, and therefore that $P \unlhd L[\mathbb{E}]$.

Now suppose that $\rho$ is not a cardinal of $L[\mathbb{E}]$. Let $\gamma=\operatorname{card}^{L[\mathbb{E}]}(\rho)$. If $\rho$ is not a generator of $F$ then the previous argument adapts easily. So suppose $\rho$ is a generator of $F$. So $\operatorname{cr}(\sigma)=\rho=\left(\gamma^{+}\right)^{\operatorname{Ult}(L[\mathbb{E}], G)}$. In this case it seems that there might not be an ultra-backgrounded construction with last model $\operatorname{Ult}(L[\mathbb{E}], G) \| \xi$. Let $G^{\prime}$ be the trivial completion of $F \uparrow(\rho+1)$. Let $\xi^{\prime}=$ $\left(\rho^{+}\right)^{\operatorname{Ult}\left(L[\mathbb{E}], G^{\prime}\right)}$. Then $\operatorname{Ult}\left(L[\mathbb{E}], G^{\prime}\right)\left\|\xi^{\prime}=L[\mathbb{E}]\right\| \xi^{\prime}$ and $\gamma$ is the largest cardinal of $L[\mathbb{E}] \| \xi^{\prime}$. So there is an ultra-backgrounded construction with last model $L[\mathbb{E}] \| \xi^{\prime}$. Let $P=\left(L[\mathbb{E}] \| \xi^{\prime}, G^{\prime}\right)$. Then there is a pseudo-ultra-backgrounded construction with last model $P$. By Claim 5 below, $P$ is $\left(0, \omega_{1}, \omega_{1}+1\right)^{*}$-iterable in $W$. So by $[3, \S 10]$ (combined with the generalization of the latter using the weak Dodd-Jensen property), $P$ is a premouse. Therefore either $G \in \mathbb{E}$, or $L[\mathbb{E}] \mid \rho$ is active and $G \in \mathbb{E}\left(\operatorname{Ult}\left(L[\mathbb{E}] \mid \rho, F^{L[\mathbb{E}] \mid \rho}\right)\right)$, as required.

The following claim completes the proof of the theorem, as it establishes the iterability we have used above, and part (c). Most of the rest of the paper is devoted to its proof; we focus on one representative case of it:

Claim 5. We have:

(i) For any $\lambda \in \mathrm{OR}^{W}$ and ultra-backgrounded construction $\mathbb{C}=\left\langle S_{\alpha}\right\rangle_{\alpha \leq \lambda}$ of $W$, and $n<\omega, \mathfrak{C}_{n}\left(S_{\lambda}^{\mathbb{C}}\right)$ exists and is $\left(n, \omega_{1}, \omega_{1}+1\right)^{*}$-iterable.

(ii) The bicephalus $C$ defined in the proof of Claim 1 is $\left(\omega_{1}+1\right)$-iterable.

(iii) For any ultra-backgrounded pseudo-construction of $W$, with last model $P, P$ is $\left(0, \omega_{1}, \omega_{1}+1\right)^{*}$-iterable.

(iv) If there is a class wellorder $<^{W}$ of $W$ then $L[\mathbb{E}]$ is $\left(0, \omega_{1}, \omega_{1}+1\right)^{*}$-iterable.

Proof. We focus on the the iterability of $C=\left(\rho^{C}, M, N\right)$ (part (ii)); parts (i) and (iii) are mostly simplifications of this. At the end we state some adaptations used for (iv). The main difference between the present iterability proof and that for a standard $L[\mathbb{E}]$-construction is in the resurrection process. The details of this process will be dealt with in a manner similar to that in [10], and moreover, the resurrection process of [10] will need to be folded into the present one. We follow the iterability proof of [10] closely. In one regard, the present proof is slightly simpler: in [10], arbitrary standard trees were considered, whereas here we deal with a more restricted class (roughly, normal) of trees.

In the pm-ultra-backgrounded setting, i.e. the proof of 6.19 , the natural adaptation of the proof to follow lifts a tree on $C$ to an almost normal tree $\mathcal{U}$ on $W$. We leave the verification of this to the reader. Likewise, its adaptation to stacks of normal trees on $\mathfrak{C}_{n}\left(S_{\alpha}^{\mathbb{C}}\right)$ and $P$ (parts (i) and (iii)) produces stacks of 
almost normal trees on $W$. This ensures that we only use the $\left(\omega, \omega_{1}, \omega_{1}+1\right)^{*}$ iterability of $W$ in this context. We ensure that this works by our arrangement of the proof to follow, which increases the work involved a little. For the adaptations to 6.19 , one should use background extenders $G$ with $\operatorname{lh}(G)$ minimal (when witnessing 6.4(3b)), and use pm-nice witnesses (but when the pm-nice witness is as in 6.12 (ii), one must use the two extenders $G_{1}$ and $G_{2}$ in $\mathcal{U}$ ).

Let $\Sigma_{W}$ be an iteration strategy for $W$. We will describe a strategy $\Sigma_{C}$ for player II in the $\left(\omega_{1}+1\right)$-iteration game on $C$. Let $\mathcal{T}$ be an iteration tree on $C$ which is via $\Sigma_{C}$. Then we will have inductively constructed a tree $\mathcal{U}$ on $W$, via $\Sigma_{W}$, such that $\mathcal{T}$ lifts to $\mathcal{U}$ (in a manner to be specified), and if $\mathcal{T}$ has limit length, we will use $\Sigma_{W}(\mathcal{U})$ to define $\Sigma_{C}(\mathcal{T})$.

We say that an iteration tree $\mathcal{V}$ on $W$ is neat iff $\mathcal{V}$ is non-overlapping and $\operatorname{str}^{M_{\alpha}^{\mathcal{V}}}\left(E_{\alpha}^{\mathcal{V}}\right) \leq \operatorname{str}^{M_{\beta}^{\mathcal{V}}}\left(E_{\beta}^{\mathcal{V}}\right)$ for $\alpha<\beta$. The tree $\mathcal{U}$ may use padding, but the tree $\mathcal{V}$ given by removing all padding from $\mathcal{U}$ will be neat. (So in the adaptation to the proof of $6.19, \mathcal{V}$ would be almost normal.)

We will have $\operatorname{lh}(\mathcal{U}) \geq \operatorname{lh}(\mathcal{T})$, but $\ln (\mathcal{U})>\operatorname{lh}(\mathcal{T})$ is possible. For each node $\alpha$ of $\mathcal{T},(\alpha, 0)$ will be a node of $\mathcal{U}$, and the model $M_{\alpha 0}^{\mathcal{U}}$ will correspond directly to $B_{\alpha}^{\mathcal{T}}$. However, there may also be a further finite set of nodes $(\alpha, i)$ of $\mathcal{U}$, and models $M_{\alpha i}^{\mathcal{U}}$ associated to initial segments of $M_{\alpha}^{\mathcal{T}}$ or $N_{\alpha}^{\mathcal{T}}$. For indexing, let $\mathrm{OR}^{*}=\mathrm{OR} \times \omega$; we order $\mathrm{OR}^{*}$ lexicographically. We index the nodes of $\mathcal{U}$ with elements of a set $\operatorname{dom}(\mathcal{U}) \subseteq \mathrm{OR}^{*}$, such that for some sequence $\left\langle k_{\alpha}\right\rangle_{\alpha<\operatorname{lh}(\mathcal{T})}$ of integers $k_{\alpha} \geq 1$, we have $(\alpha, i) \in \operatorname{dom}(\mathcal{U})$ iff $\alpha<\operatorname{lh}(\mathcal{T})$ and $i<k_{\alpha}$. So if $\operatorname{lh}(\mathcal{T})>1$ then $\operatorname{dom}(\mathcal{U})$ is not closed downward under $<$.

For notational convenience we allow $\mathcal{U}$ to use padding. If $E=E_{\alpha i}^{\mathcal{U}}=\emptyset$ we consider $\operatorname{str}^{M_{\alpha i}^{\mathcal{U}}}(E)=\operatorname{OR}\left(M_{\alpha i}^{\mathcal{U}}\right)$; we do allow $\operatorname{pred}^{\mathcal{U}}(\beta, j)=(\alpha, i)$ in this case.

We make some preparations. Let $\alpha<\operatorname{lh}(\mathcal{T})$. Write $B_{\alpha}=B_{\alpha}^{\mathcal{T}}, M_{\alpha}=M_{\alpha}^{\mathcal{T}}$, etc. If $\alpha \in \mathscr{B}^{\mathcal{T}}$ let $\left(m_{\alpha}, n_{\alpha}\right)=\operatorname{deg}^{\mathcal{T}}(\alpha)=\left(m_{0}, n_{0}\right)$. If $\alpha \in \mathscr{M}^{\mathcal{T}}$ let $m_{\alpha}=$ $\operatorname{deg}^{\mathcal{T}}(\alpha)$. If $\alpha \in \mathscr{N}^{\mathcal{T}}$ let $n_{\alpha}=\operatorname{deg}^{\mathcal{T}}(\alpha)$. If $[0, \alpha]_{\mathcal{T}}$ does not drop in model, then:

- If $M_{\alpha} \neq \emptyset$ then $\vartheta_{\alpha}$ denotes $\psi_{i_{\alpha}^{\tau}}\left(\rho^{C}\right)$, (so if $\rho^{C}<\rho_{0}^{M}$, which is the main case of interest here, then $\left.\vartheta_{\alpha}=i_{0 \alpha}^{\mathcal{T}}\left(\rho^{C}\right)\right)$.

- If $N_{\alpha} \neq \emptyset$ then $\widetilde{\vartheta}_{\alpha}$ denotes $\psi_{j_{\alpha \alpha}^{\tau}}\left(\rho^{C}\right)$.

Recall here that if $\alpha \in \mathscr{B}^{\mathcal{T}}$ then $\rho\left(B_{\alpha}^{\mathcal{T}}\right)=\sup i_{0 \alpha}^{\mathcal{T}} " \rho^{C}=\sup j_{0 \alpha}^{\mathcal{T}} " \rho^{C}$, but these iteration maps can be discontinuous at $\rho^{C}$ and we can have $\rho\left(B_{\alpha}^{\mathcal{T}}\right)<\vartheta_{\alpha}$ and $\rho\left(B_{\alpha}^{\mathcal{T}}\right)<\widetilde{\vartheta}_{\alpha} .{ }^{34}$ We say that $\alpha$ is $\mathscr{M}$-stable iff $M_{\alpha} \neq \emptyset$ and $[0, \alpha]_{\mathcal{T}}$ does not drop and $\operatorname{cr}\left(i_{\beta \alpha}^{\mathcal{T}}\right)<\vartheta_{\beta}$ for all $\beta \in[0, \alpha)_{\mathcal{T}}$. We define $\mathscr{N}$-stable analogously. Note that if $\alpha \in \mathscr{M}^{\mathcal{T}}$ is $\mathscr{M}$-stable then $\rho^{C}<\rho_{0}^{M}$ and $i_{0 \beta}^{\mathcal{T}}$ is discontinuous at $\rho^{C}$, where $\beta=\max \left([0, \alpha)_{\mathcal{T}} \cap \mathscr{B}^{\mathcal{T}}\right)$ (since then in fact $\rho\left(B_{\beta}^{\mathcal{T}}\right) \leq \operatorname{cr}\left(i_{\beta \alpha}^{\mathcal{T}}\right)<\vartheta_{\beta}$, and in particular $\rho\left(B_{\beta}^{\mathcal{T}}\right)<\rho_{0}\left(M_{\beta}\right)$ ). We are only interested in $\vartheta_{\alpha}$ for $\mathscr{M}$-stable $\alpha$.

\footnotetext{
${ }^{34}$ Actually, if $\alpha \in \mathscr{B}^{\mathcal{T}}$ then $\vartheta_{\alpha}=\widetilde{\vartheta}_{\alpha}$ and $M_{\alpha}\left\|\left(\vartheta_{\alpha}^{+}\right)^{M_{\alpha}}=N_{\alpha}\right\|\left(\vartheta_{\alpha}^{+}\right)^{N_{\alpha}}$, but this is not important.
} 
Let $\mathbb{C}_{\alpha i}=i_{00, \alpha i}^{\mathcal{U}}(\mathbb{C})$ and $\Gamma_{\alpha i}=\operatorname{lh}\left(\mathbb{C}_{\alpha i}\right)$. Let $\widetilde{\mathbb{C}}_{\alpha i}=i_{00, \alpha i}^{\mathcal{U}}(\widetilde{\mathbb{C}})$ and $\widetilde{\Gamma}_{\alpha i}=$ $\operatorname{lh}\left(\widetilde{\mathbb{C}}_{\alpha i}\right)$. When we say, for example, $\mathbb{C}_{\alpha i}$-standard we literally mean $\mathbb{C}_{\alpha i}$-standard in $M_{\alpha i}^{\mathcal{U}}$. Let $\operatorname{str}_{\alpha i}=\operatorname{str}^{M_{\alpha i}^{\mathcal{U}}}\left(E_{\alpha i}^{\mathcal{U}}\right)$. We will also associate later an ordinal $s_{\alpha i} \leq \operatorname{str}_{\alpha i}$.

We make some arrangements to help us choosing background extenders for $\mathcal{U}$ (recall we do not assume AC in $V$ ). We have $W \models \mathrm{ZFC}$. Fix $\xi \in \mathrm{OR}^{W}$ with $\mathbb{C}, \widetilde{\mathbb{C}} \in V_{\xi}^{W}$ and such that $V_{\xi}^{W} \preccurlyeq k W$ for a sufficiently large $k$. In particular, whenever $W$ has a background extender for $S_{\alpha}^{\mathbb{C}}$ or $S_{\alpha}^{\widetilde{\mathbb{C}}}$, then $V_{\xi}^{W}$ has one too. Let $<^{*} \in W$ be a wellorder of $V_{\xi}^{W}$. Given $(\beta, j) \in \operatorname{dom}(\mathcal{U})$ we write $<_{\beta j}^{*}=$ $i_{00, \beta j}^{\mathcal{U}}\left(<^{*}\right)$. We will use $<_{\beta j}^{*}$ in determining $E_{\beta j}^{\mathcal{U}}$. Let $\mathscr{E} \subseteq M_{\beta j}^{\mathcal{U}}$ be a nonempty collection of $M_{\beta j}^{\mathcal{U}}$-extenders. Let $s_{0}=\min _{E \in \mathscr{E}} \operatorname{str}^{M_{\beta j}^{U}}(E)$. Then the *-least element of $\mathscr{E}$ is the $<_{\beta j}^{*}$-least $E \in \mathscr{E}$ such that $\operatorname{str}^{M_{\beta j}^{u}}(E)=s_{0}$ and $E$ is Mitchell-minimal such; that is, $\mathscr{E} \cap \operatorname{Ult}\left(M_{\beta j}^{\mathcal{U}}, E\right)=\emptyset$. Given a property $\varphi$, the *-least extender $E$ such that $\varphi(E)$ means *-least element of $\{E \mid \varphi(E)\}$.

Let $\uparrow \notin$ OR (here "个" means undefined). Let $\vec{\xi}, \vec{\zeta} \in(\mathrm{OR} \cup\{\uparrow\})^{2} \backslash\{(\uparrow, \uparrow)\}$ and let $\vec{\xi}=(\xi, \widetilde{\xi})$ and $\vec{\zeta}=(\zeta, \widetilde{\zeta})$. For $\gamma \in$ OR let $\max (\gamma, \uparrow)=\max (\uparrow, \gamma)=\gamma$. We write $\vec{\xi}<\vec{\zeta}$ iff $\uparrow \in \vec{\xi}$ and $\max (\vec{\xi}) \leq \max (\vec{\zeta})$ and if $\uparrow \in \vec{\zeta}$ then $\max (\vec{\xi})<\max (\vec{\zeta})$. Clearly this order is wellfounded.

6.22 Definition. Let $D$ be a premouse and $\gamma \leq \mathrm{OR}^{D}$. The $\gamma$-dropdown sequence of $D$ is the sequence $\sigma=\left\langle\left(D_{i}, \delta_{i}\right)\right\rangle_{i<n}$ of maximum length such if $\gamma=\mathrm{OR}^{D}$ then $\sigma=\emptyset$, and if $\gamma<\mathrm{OR}^{D}$ then $D_{0}=M \mid \gamma$, and for each $i<n$, $\delta_{i}=\rho_{\omega}\left(D_{i}\right)$, and if $i+1<n$ then $D_{i+1}$ is the least $A$ such that $D_{i} \triangleleft A \triangleleft D$ and $\rho_{\omega}^{A}<\delta_{i}$.

Suppose $M_{\alpha} \neq \emptyset$ and let $\gamma \leq \operatorname{OR}\left(M_{\alpha}\right)$. The $(\mathcal{T}, \alpha, \gamma)$-dropdown sequence $\tau$ of $M_{\alpha}$ is defined as follows. Let $\sigma$ be the $\gamma$-dropdown sequence of $M_{\alpha}$. Then:

(a) if $\alpha$ is $\mathscr{M}$-stable and $\vartheta_{\alpha}<\mathrm{OR}^{M_{\alpha}}$ and $\left(\vartheta_{\alpha}^{+}\right)^{M_{\alpha}} \leq \gamma^{35}$ then

$$
\tau=\sigma^{\curlywedge}\left\langle\left(M_{\alpha}, \vartheta_{\alpha}\right),\left(M_{\alpha}, 0\right)\right\rangle
$$

(b) otherwise $\tau=\sigma^{\wedge}\left\langle\left(M_{\alpha}, 0\right)\right\rangle$.

If $N_{\alpha} \neq \emptyset$ then for $\gamma \leq \operatorname{OR}\left(N_{\alpha}\right)$, we define the $(\mathcal{T}, \alpha, \gamma)$-dropdown sequence of $N_{\alpha}$ analogously.

6.23 Remark. Suppose $\alpha+1<\operatorname{lh}(\mathcal{T})$ and $E_{\alpha}^{\mathcal{T}} \in \mathbb{E}_{+}\left(M_{\alpha}\right)$. Let $\tau^{*}=\left\langle\left(M_{i}, \varrho_{i}\right)\right\rangle_{i \leq n^{\prime}}$ be the reverse of the $\left(\mathcal{T}, \alpha, \operatorname{lh}\left(E_{\alpha}^{\mathcal{T}}\right)\right)$-dropdown sequence $\tau$ (the same sequence but in reversed order). Note that $M_{0}=M_{\alpha}, M_{n^{\prime}}=\operatorname{ex}_{\alpha}^{\mathcal{T}}, \varrho_{0}^{\prime}=0$ and $\varrho_{i}^{\prime}<\varrho_{i+1}^{\prime}$ for all $i+1 \leq n^{\prime}$. Note that if $\beta+1<\operatorname{lh}(\mathcal{T})$ and $\alpha=\operatorname{pred}^{\mathcal{T}}(\beta+1)$ then $M_{\beta+1}^{* \mathcal{T}}=M_{i}$ for some $i \leq n^{\prime}$.

\footnotetext{
${ }^{35}$ So $\vartheta_{\alpha}<\left(\vartheta_{\alpha}^{+}\right)^{M_{\alpha}} \leq \varrho_{i}$ for each $i<\operatorname{lh}(\sigma)$.
} 
6.24 Remark. We now give a sketch of the resurrection process and the meaning of $\mathcal{U} \uparrow\left[(\delta, 0),\left(\delta, k_{\delta}-1\right)\right]$. Figures 1 and 2 depict various features discussed here, under certain further simplifying assumptions, but they incorporate more details which will be explained later. In Figure 1 it happens that we do not need to take ultrapowers at even stages of the resurrection (see below), so there the diagram looks more like standard resurrection, although there can be ultrapowers taken at odd stages (see below).

For simplicity, we assume for the duration of the sketch that $\delta \in \mathscr{M}^{\mathcal{T}}$ is non- $\mathscr{M}$-stable, but in other cases things are similar. Then we will have $k_{\delta}=$ $2 u_{\delta}+1$ where the reversed $\left(\mathcal{T}, \delta, \operatorname{lh}\left(E_{\delta}\right)\right)$-dropdown sequence is $\left\langle\left(M_{\delta i}, \varrho_{\delta i}\right)\right\rangle_{i \leq u_{\delta}}$. If $u_{\delta}>0$, then the extenders

$$
E_{\delta 0}^{\mathcal{U}}, E_{\delta 1}^{\mathcal{U}}, \ldots, E_{\delta, 2 u_{\delta}-1}^{\mathcal{U}}
$$

will facilitate the resurrection process used to find a background extender $E^{*}$ into which we can embed $E_{\delta}$, and then we will set

$$
E_{\delta, 2 u_{\delta}}^{\mathcal{U}}=E^{*} \neq \emptyset \text {. }
$$

It is possible that $E_{\delta j}^{\mathcal{U}}=\emptyset$ for $j<2 u_{\delta}$. The resurrection will yield for each $i<u_{\delta}$, a sound model $\mathfrak{C}_{\omega}\left(R_{\delta, i+1}\right)$, constructed (by an ultrabackgrounded construction) in $M_{\delta, 2 i+1}^{\mathcal{U}}$, and a fully elementary

$$
\pi_{\delta, i+1}: \mathfrak{C}_{0}\left(M_{i+1}\right) \rightarrow \mathfrak{C}_{\omega}\left(R_{\delta, i+1}\right),
$$

and will also yield $Q_{\delta, i+1}$, constructed at a standard stage in $M_{\delta, 2 i+2}^{\mathcal{U}}$, with $\mathfrak{C}_{\omega}\left(Q_{\delta, i+1}\right)=\mathfrak{C}_{\omega}\left(R_{\delta, i+1}\right)$, and thus, we also embed $M_{i+1}$ into $Q_{\delta, i+1}$. If $i<u_{\delta}$ and $E_{\delta, 2 i}^{\mathcal{U}} \neq \emptyset$, then $M_{i}$ is type 3 and $\varrho_{i+1}=\nu\left(M_{i}\right)$, and it happens that the standard resurrection process fails to yield an appropriate model $R_{\delta, i+1}$. That is, we will have already found $Q_{\delta i} \in M_{\delta, 2 i}^{\mathcal{U}}$ and $\sigma: \mathfrak{C}_{0}\left(M_{i}\right) \rightarrow \mathfrak{C}_{0}\left(Q_{\delta i}\right)$. So $M_{i+1} \notin M_{i}^{\text {sq }}=\operatorname{dom}(\sigma)$, so $\sigma$ does not act directly on $M_{i+1}$. If $\sigma$ is non- $\nu$-high then $\psi_{\sigma}\left(M_{i+1}\right) \triangleleft Q_{\delta i}$, in which case we can set $E_{\delta, 2 i}^{\mathcal{U}}=\emptyset$ and $\mathfrak{C}_{\omega}\left(R_{\delta, i+1}\right)=$ $\psi_{\sigma}\left(M_{i+1}\right)$ (this is basically the standard resurrection process). But if $\sigma$ is $\nu$ high, then $\psi_{\sigma}\left(M_{i+1}\right)$ is not a stage of the construction in $M_{\alpha, 2 i}^{\mathcal{U}}$; in this case we set $E_{\delta, 2 i}^{\mathcal{U}}$ to be a background extender for $F\left(Q_{\delta i}\right)$, and this will actually ensure that an appropriate $R_{\delta, i+1}$ appears in $M_{\delta, 2 i+1}^{\mathcal{U}}$ (with $\mathfrak{C}_{\omega}\left(R_{\delta, i+1}\right)$ either $=\psi_{\sigma}\left(M_{i+1}\right)$ or some variant thereof). We will write $s_{\delta, 2 i}=\nu\left(F\left(Q_{\delta i}\right)\right)$ here; if $E_{\delta, 2 i}^{\mathcal{U}}=\emptyset$ then we set $s_{\delta, 2 i}=s_{\delta, 2 i+1}$. If $i<u_{\delta}$ and $E_{\delta, 2 i+1}^{\mathcal{U}} \neq \emptyset$, then $R_{\delta, i+1}$ was constructed by a non-standard stage in $M_{\delta, 2 i+1}^{\mathcal{U}}$, and $E_{\delta, 2 i+1}^{\mathcal{U}}$ is then selected witnessing $6.3(3 \mathrm{c})$ for $R_{\delta, i+1}$, yielding an appropriate $Q_{\delta, 2 i+1}$ in $M_{\delta, 2 i+2}^{\mathcal{U}}$, and we proceed with the next step of resurrection (uncoring) there. In this case we set $s_{\delta, 2 i+1}=\rho_{\omega}\left(R_{\delta, i+1}\right)$. This eventually leads (including in the case that $u_{\delta}=0$ ) to a model $Q_{\delta}^{*}=Q_{\delta, 2 u_{\delta}} \in M_{\delta, 2 u_{\delta}}^{\mathcal{U}}$ and 0-lifting embedding

$$
\pi_{\delta}^{*}: \mathfrak{C}_{0}\left(\operatorname{ex}_{\delta}^{\mathcal{T}}\right) \rightarrow \mathfrak{C}_{0}\left(Q_{\delta}^{*}\right) .
$$

If $\pi_{\delta}^{*}$ is non- $\nu$-low then we set $E_{\delta, 2 u_{\delta}}^{\mathcal{U}}=E^{*}$ to be an appropriate background for $F\left(Q_{\delta}^{*}\right)$, and $s_{\delta, 2 u_{\delta}}=\nu\left(Q_{\delta}^{*}\right)$, whereas if $\pi_{\delta}^{*}$ is $\nu$-low and $\nu^{\prime}=\psi_{\pi_{\delta}^{*}}\left(\nu_{\delta}^{\mathcal{T}}\right)$ then we 


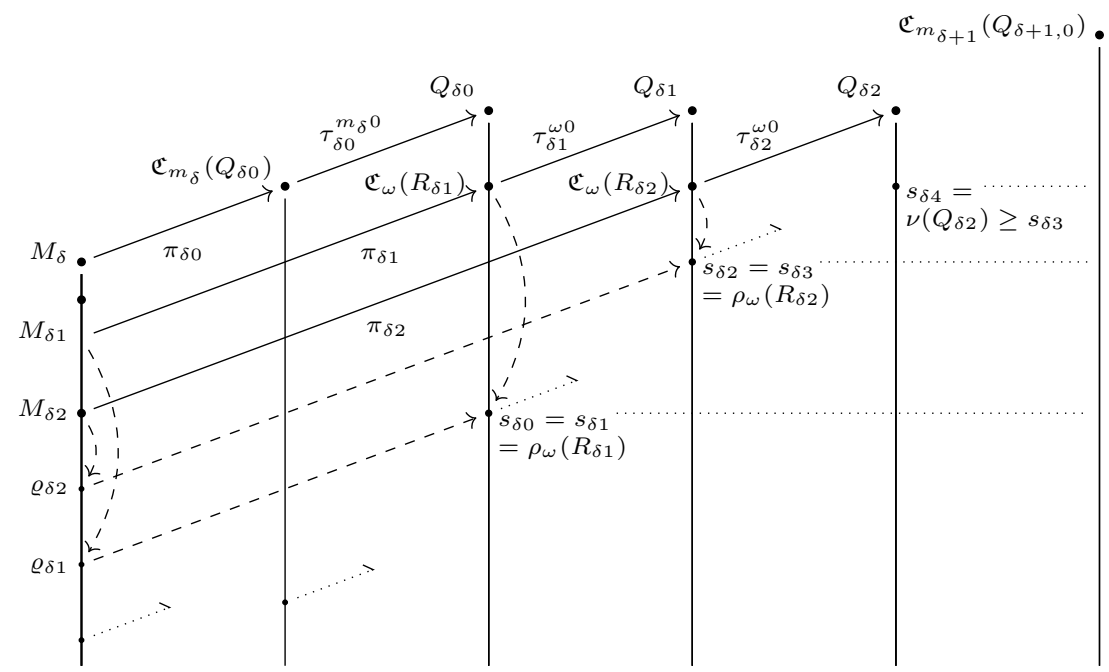

Figure 1: Dropdown resurrection and associated objects, in the case that $\delta \in \mathscr{M}^{\mathcal{T}}$ is non$\mathscr{M}$-stable, $u_{\delta}=2$, and all models are non-type 3 . Vertical lines indicate ordinals, with height roughly corresponding to ordinal rank. Solid arrows denote embeddings $\left(\pi_{\delta 0}\right.$ etc), with domains and codomains denoted by large bullets (except that the domain is literally the squash). Small bullets indicate the positions of labelled ordinals ( $\varrho_{\delta 1}$ etc). Dashed arrows indicate trajectories under embeddings. The bases of the dotted half-arrows indicate approximately the critical points of $\pi_{\delta 0}, \tau_{\delta 0}^{m_{\delta} 0}, \tau_{\delta 1}^{\omega 0}, \tau_{\delta 2}^{\omega 0}$ respectively; the main point here is that $\operatorname{cr}\left(\tau_{\delta, i+1}^{\omega 0}\right) \geq s_{\delta, 2 i+1}$. Curved dashed arrows indicate positions of $\omega$ th projecta $\rho_{\omega}^{N}$ of models $N$. Dotted horizontal lines indicate agreement between models $N_{1}, N_{2}$ strictly below that ordinal $\alpha$; that is, $N_{1}\left\|\alpha=N_{2}\right\| \alpha$. (If $\alpha<s_{\delta 4}$ then in fact, $\alpha$ is also a cardinal of both models and so $N_{1}\left|\alpha=N_{2}\right| \alpha$. $)$ Note $\mathfrak{C}_{\omega}\left(R_{\delta, i+1}\right)=\mathfrak{C}_{\omega}\left(Q_{\delta, i+1}\right)$. Note $s_{\delta 1}<s_{\delta 2}$ and $s_{\delta 3} \leq s_{\delta 4}$, but $s_{\delta 3}=s_{\delta 4}$ is possible. Note that because $\operatorname{ex}_{\delta}^{\mathcal{T}}$ is non-type 3 , we have $s_{\delta 4}=\nu\left(Q_{\delta 2}\right)$.

do likewise but with $Q^{\prime} \triangleleft Q_{\delta}^{*}$ instead of $Q_{\delta}^{*}$, where $Q^{\prime}$ is chosen as in 1.1 with respect to $\pi_{\delta}^{*}$. This completes the sketch of resurrection.

We now proceed with the details. We first state some intentions, introduce more notation, and state hypotheses (L1)-(L12), to be maintained by induction on initial segments of $(\mathcal{T}, \mathcal{U})$. Let $\alpha<\operatorname{lh}(\mathcal{T})$. If $M_{\alpha} \neq \emptyset$ we will define:

$$
\begin{aligned}
& -\mathbb{D}_{\alpha 0} \in\left\{\mathbb{C}_{\alpha 0}, \widetilde{\mathbb{C}}_{\alpha 0}\right\}, \\
& -\Delta_{\alpha 0}=\operatorname{lh}\left(\mathbb{D}_{\alpha 0}\right), \\
& -\xi_{\alpha 0}<\Delta_{\alpha 0}, \\
& -\left(M_{\alpha 0}, m_{\alpha 0}\right)=\left(M_{\alpha}, m_{\alpha}\right) \text { and } Q_{\alpha 0}=S_{\xi_{\alpha 0}}^{\mathbb{D}_{\alpha 0}}, \\
& \text { - and a c-preserving } m_{\alpha 0} \text {-lifting } \pi_{\alpha 0}: \mathfrak{C}_{0}\left(M_{\alpha 0}\right) \rightarrow \mathfrak{C}_{m_{\alpha 0}}\left(Q_{\alpha 0}\right)
\end{aligned}
$$

such that: 


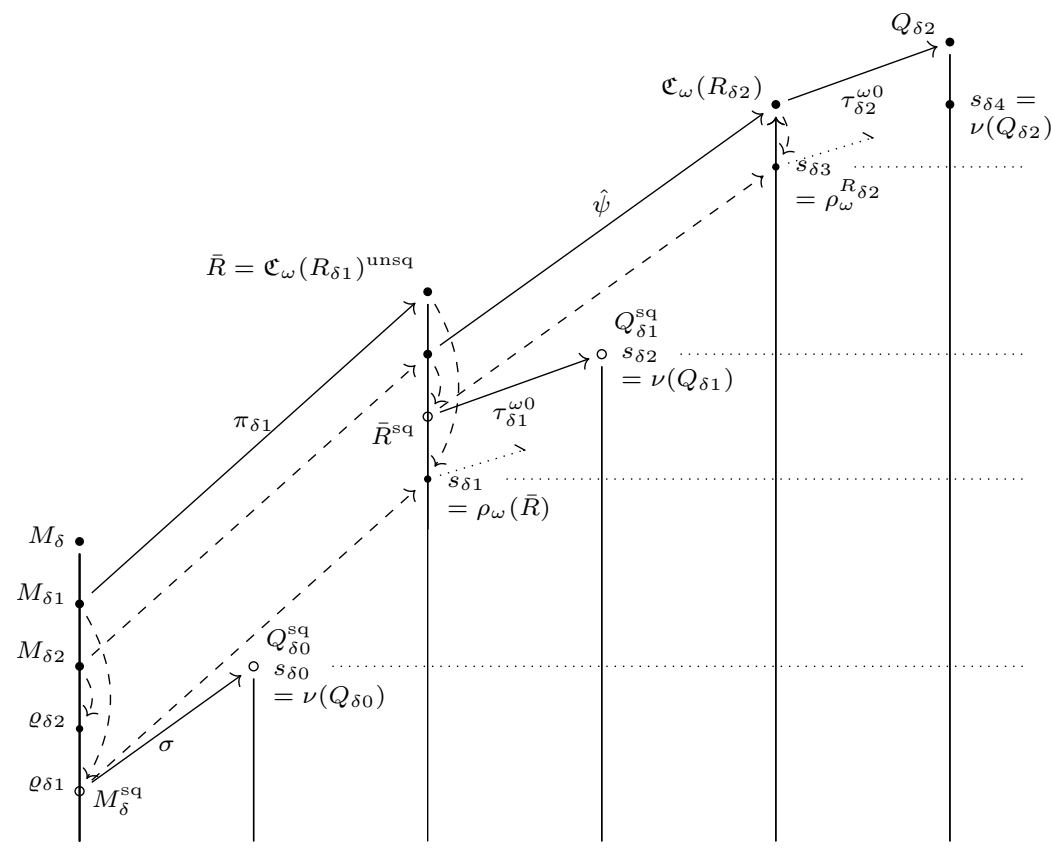

Figure 2: Dropdown resurrection and associated objects, in the case that $\delta \in \mathscr{M}^{\mathcal{T}}$ is non$\mathscr{M}$-stable, $u_{\delta}=2, E_{\delta j}^{\mathcal{U}} \neq \emptyset$ for each $j<4$, and $M_{\delta 2}$ is non-type 3 ; hence, $M_{\delta i}$ is type 3 and $\nu\left(M_{\delta i}\right)=\varrho_{\delta, i+1}$ for $i=0,1$. Notation is as in Figure 1; also, an open circle denotes the height of a squashed premouse, which is also the domain or codomain of $\sigma$ or $\tau_{\delta 1}^{\omega 0}$. Note $\sigma=\tau_{\delta 0}^{m_{\delta} 0} \circ \pi_{\delta 0}$ and $\hat{\psi}$ is defined as in Subsubcase 3.2.2 in the inductive construction of $\mathcal{U}$ to follow (so $\pi_{\delta 2}=\hat{\psi} \circ \psi_{\pi_{\delta 1}}\left\lceil\mathfrak{C}_{0}\left(M_{\delta 2}\right)\right.$ ). Note that $s_{\delta 0}<s_{\delta 1}<s_{\delta 2}<s_{\delta 3}<s_{\delta 4}$ (because $E_{\delta j}^{\mathcal{U}} \neq \emptyset$ for each $j<4)$. Note that the models $\mathfrak{C}_{m_{\delta}}\left(Q_{\delta 0}\right)$ and $\mathfrak{C}_{m_{\delta+1}}\left(Q_{\delta+1,0}\right)$ have been omitted from this figure, though they are present in Figure 1. Note that we again have $s_{\delta 4}=\nu\left(Q_{\delta 2}\right)$ because $M_{\delta 2}=\operatorname{ex}_{\delta}^{\mathcal{T}}$ is non-type 3 . 
(L1) If $\alpha$ is $\mathscr{M}$-stable then $\mathbb{D}_{\alpha 0}=\mathbb{C}_{\alpha 0}$ and $\xi_{\alpha 0}=i_{00, \alpha 0}^{\mathcal{U}}\left(\lambda^{\mathbb{C}}\right)$ and

$$
\pi_{\alpha 0} \circ i_{0 \alpha}^{\mathcal{T}}=i_{00, \alpha 0}^{\mathcal{U}}\left\lceil\mathfrak{C}_{0}(M)\right.
$$

so if $\rho^{C}<\rho_{0}^{M}$ then $\pi_{\alpha 0}\left(\vartheta_{\alpha}\right)=i_{00, \alpha 0}^{\mathcal{U}}\left(\rho^{C}\right)$.

(L2) If $\alpha$ is non- $\mathscr{M}$-stable then $\xi_{\alpha 0}$ is $\mathbb{D}_{\alpha 0}$-standard.

If $N_{\alpha} \neq \emptyset$ we will define $\widetilde{\mathbb{D}}_{\alpha 0}, \widetilde{\Delta}_{\alpha 0}, \widetilde{\xi}_{\alpha 0}, \widetilde{Q}_{\alpha 0}$ and $\widetilde{\pi}_{\alpha 0}$ analogously (and maintain analogous properties).

Now suppose $\alpha+1<\operatorname{lh}(\mathcal{T})$.

Suppose that $E_{\alpha} \in \mathbb{E}_{+}\left(M_{\alpha}\right)$. Let $\left\langle\left(M_{\alpha i}, \varrho_{\alpha i}\right)\right\rangle_{i<u_{\alpha}}$ be the reverse of the $\left(\mathcal{T}, \alpha, \operatorname{lh}\left(E_{\alpha}\right)\right)$-dropdown sequence of $M_{\alpha}$. Then $k_{\alpha}=2 u_{\alpha}+1$. Fix $i \leq u_{\alpha}$. Let $m_{\alpha i}=m_{\alpha}$ if $M_{\alpha i}=M_{\alpha}$ and $m_{\alpha i}=\omega$ otherwise (in fact we already defined $M_{\alpha 0}$ and $m_{\alpha 0}$ above). If $i>0$ then for each $j \in\{2 i-1,2 i\}$ we will define:

$-\mathbb{D}_{\alpha j} \in\left\{\mathbb{C}_{\alpha j}, \widetilde{\mathbb{C}}_{\alpha j}\right\}$,

- $\Delta_{\alpha j}=\operatorname{lh}\left(\mathbb{D}_{\alpha j}\right)$,

$-\xi_{\alpha j}<\Delta_{\alpha j}$,

- $R_{\alpha i}=S_{\xi_{\alpha, 2 i-1}}^{\mathbb{D}_{\alpha, 2 i-1}}$ and $Q_{\alpha i}=S_{\xi_{\alpha, 2 i}}^{\mathbb{D}_{\alpha, 2 i}}$, and

- a c-preserving $m_{\alpha i}$-lifting embedding $\pi_{\alpha i}: \mathfrak{C}_{0}\left(M_{\alpha i}\right) \rightarrow \mathfrak{C}_{m_{\alpha i}}\left(Q_{\alpha i}\right)$

such that:

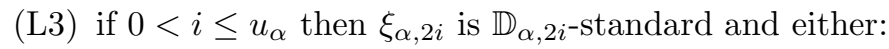

- $\mathfrak{C}_{m_{\alpha i}}\left(R_{\alpha i}\right)=\mathfrak{C}_{m_{\alpha i}}\left(Q_{\alpha i}\right)$, or

- $M$ is non-standard (in $W$ ), the hypothesis of Definition 6.22(a) holds for the $\left(\mathcal{T}, \alpha, \operatorname{lh}\left(E_{\alpha}\right)\right)$-dropdown (that is, $\alpha$ is $\mathscr{M}$-stable and $\left(\vartheta_{\alpha}^{+}\right)^{M_{\alpha}} \leq$ $\operatorname{lh}\left(E_{\alpha}\right)$, so $\left.M_{\alpha 1}=M_{\alpha 0}=M_{\alpha}\right), i=1, \mathbb{D}_{\alpha 0}=\mathbb{C}_{\alpha 0}=\mathbb{C}_{\alpha 1}=\mathbb{D}_{\alpha 1}$, $m_{\alpha 1}=m_{\alpha 0}=m_{\alpha}=m_{0}$ and $\mathfrak{C}_{m_{0}+1}\left(Q_{\alpha 1}\right)=\mathfrak{C}_{0}\left(R_{\alpha 1}\right)=\mathfrak{C}_{0}\left(Q_{\alpha 0}\right)$ is fully sound.

For $m \leq n \leq m_{\alpha i}$ let

$$
\tau_{\alpha i}^{n m}: \mathfrak{C}_{n}\left(Q_{\alpha i}\right) \rightarrow \mathfrak{C}_{m}\left(Q_{\alpha i}\right)
$$

be the core embedding. Let $Q_{\alpha}^{*}=Q_{\alpha u_{\alpha}}$ and $m=m_{\alpha u_{\alpha}}$ and

$$
\begin{gathered}
\pi_{\alpha}^{*}: \mathfrak{C}_{0}\left(\operatorname{ex}_{\alpha}^{\mathcal{T}}\right) \rightarrow \mathfrak{C}_{0}\left(Q_{\alpha}^{*}\right), \\
\pi_{\alpha}^{*}=\tau_{\alpha u_{\alpha}}^{m 0} \circ \pi_{\alpha u_{\alpha}} .
\end{gathered}
$$

If $\pi_{\alpha}^{*}$ is $\nu$-low then let $Q_{\alpha}^{\prime}$ be derived from $\pi_{\alpha}^{*}$ as in 1.1, and otherwise let $Q_{\alpha}^{\prime}=Q_{\alpha}^{*}$. Let $c_{\alpha}$ be the set of infinite ex ${ }_{\alpha}^{\mathcal{T}}$-cardinals $\kappa<\nu(E)$. Fix $\kappa \in c_{\alpha}$. Let $i_{\alpha \kappa}$ be the largest $i$ such that $\varrho_{\alpha i} \leq \kappa$. Let $i=i_{\alpha \kappa}$. Let $m_{\alpha \kappa}$ be the least $m$ such that either 
- $M_{\alpha i}=M_{\alpha}$ and $m=m_{\alpha}$, or

$-\rho_{m+1}\left(M_{\alpha i}\right) \leq \kappa$.

Let $M_{\alpha \kappa}=M_{\alpha i}$. We define the c-preserving $m_{\alpha \kappa}$-lifting embedding

$$
\pi_{\alpha \kappa}: \mathfrak{C}_{0}\left(M_{\alpha \kappa}\right) \rightarrow \mathfrak{C}_{m_{\alpha \kappa}}\left(Q_{\alpha i}\right)
$$

by $\pi_{\alpha \kappa}=\tau_{\alpha i}^{n m} \circ \pi_{\alpha i}$, where $n=m_{\alpha i}$ and $m=m_{\alpha \kappa}$. If $\alpha \in \mathscr{B}^{\mathcal{T}}$ and $\kappa<\rho_{\alpha}$ and $\left(\kappa^{+}\right)^{B_{\alpha}}<\operatorname{lh}(E)$ then we also define $N_{\alpha \kappa}=N_{\alpha}, n_{\alpha \kappa}=n_{\alpha}$, and $\widetilde{\pi}_{\alpha \kappa}=\widetilde{\pi}_{\alpha 0}$.

Now suppose instead that $E \in \mathbb{E}_{+}\left(N_{\alpha}\right) \backslash \mathbb{E}_{+}\left(M_{\alpha}\right)$. Then we make symmetric definitions by analogy to the preceding ones. (So for example, we let $\sigma$ be the $(\mathcal{T}, \alpha, \operatorname{lh}(E))$-dropdown sequence of $N_{\alpha}$, and set $u_{\alpha}+1=\operatorname{lh}(\sigma)$, and for $i \leq u_{\alpha}$ we define $N_{\alpha i}$ and $n_{\alpha i}$, and also define $\widetilde{\xi}_{\alpha i}, \widetilde{Q}_{\alpha i}$, etc.)

Let $\omega_{\alpha}^{*}=\pi_{\alpha}^{*}$ or $\omega_{\alpha}^{*}=\widetilde{\pi}_{\alpha}^{*}$, whichever is defined. Let $\vec{\xi}_{\alpha i}=\left(\xi_{\alpha i}, \widetilde{\xi}_{\alpha i}\right)$, where if $\widetilde{\xi}_{\alpha i}$ is undefined then $\vec{\xi}_{\alpha i}=\left(\xi_{\alpha i}, \uparrow\right)$, etc.

We now list the remaining inductive hypotheses:

(L4) Let $\beta+1<\operatorname{lh}(\mathcal{T})$ and $\alpha=\operatorname{pred}^{\mathcal{T}}(\beta+1)$. Then:

(a) If $\beta+1$ is $\mathscr{M}$-stable or $\mathscr{N}$-stable then $\operatorname{pred}^{\mathcal{U}}(\beta+1,0)=(\alpha, 0)$.

(b) Suppose $\beta+1 \in \mathscr{M}^{\mathcal{T}}$ is non- $\mathscr{M}$-stable and let $i \leq u_{\alpha}$ be such that $M_{\beta+1}^{*}=M_{\alpha i}$ and if $\alpha$ is $\mathscr{M}$-stable then $i \geq 1 .{ }^{36}$ Then

$$
\operatorname{pred}^{\mathcal{U}}(\beta+1,0)=(\alpha, 2 i) \text {. }
$$

(c) Likewise if $\beta+1 \in \mathscr{N}^{\mathcal{T}}$ is non- $\mathscr{N}$-stable.

(L5) Let $\alpha \leq \beta<\operatorname{lh}(\mathcal{T})$ and $j<k_{\alpha}$ and $k<k_{\beta}$ with $\beta$ or $k$ a successor, and $(\alpha, j)=\operatorname{pred}^{\mathcal{U}}(\beta, k)$. Then:

(a) $\vec{\xi}_{\beta k} \leq i_{\alpha j, \beta k}^{\mathcal{U}}\left(\vec{\xi}_{\alpha j}\right)$.

(b) If $k$ is odd then $\vec{\xi}_{\beta k}<i_{\alpha j, \beta k}^{\mathcal{U}}\left(\vec{\xi}_{\alpha j}\right)$.

(c) If $k$ is even and $k>0$ then either:

i. $(\alpha, j)=(\beta, k-1)$, so $j$ is odd and $\vec{\xi}_{\beta k}<i_{\gamma \ell, \beta k}^{\mathcal{U}}\left(\vec{\xi}_{\gamma \ell}\right)$ where $(\gamma, \ell)=\operatorname{pred}^{\mathcal{U}}(\alpha, j)$, or

ii. $\vec{\xi}_{\beta k}<i_{\alpha j, \beta k}^{\mathcal{U}}\left(\vec{\xi}_{\alpha j}\right)$.

(d) Suppose $k=0$; so $\alpha=\operatorname{pred}^{\mathcal{T}}(\beta)$ and $j=2 i$ is even (by part (L4) above). Then

$$
\left(\mathbb{D}_{\beta 0}, \widetilde{\mathbb{D}}_{\beta 0}, \vec{\xi}_{\beta 0}\right)=i_{\alpha j, \beta 0}^{\mathcal{U}}\left(\mathbb{D}_{\alpha j}, \widetilde{\mathbb{D}}_{\alpha j}, \vec{\xi}_{\alpha j}\right)
$$

\footnotetext{
${ }^{36}$ Note that if $\xi \in \mathscr{M}^{\mathcal{T}}$ is is non- $\mathscr{M}$-stable but all $\alpha<\mathcal{T} \xi$ are $\mathscr{M}$-stable, then $\xi=\beta+1$ for some $\beta$, and letting $\alpha=\operatorname{pred}^{\mathcal{T}}(\beta+1)$, then $\alpha$ is $\mathscr{M}$-stable (so $[0, \alpha]_{\mathcal{T}}$ does not drop in model), and if $\mathcal{T}$ does not drop in model at $\beta+1$ then $\vartheta_{\alpha} \leq \operatorname{cr}\left(E_{\beta}\right)$ and $E_{\beta}$ is $M_{\alpha}$-total, so $\left(\vartheta_{\alpha}^{+}\right)^{M_{\alpha}} \leq \operatorname{lh}\left(E_{\alpha}\right)$, so $M_{\alpha 1}=M_{\alpha 0}=M_{\alpha}=M_{\beta+1}^{*}$.
} 
and if $M_{\beta} \neq \emptyset$ then

$$
\pi_{\beta 0} \circ i_{\beta}^{* \mathcal{T}}=i_{\alpha j, \beta 0}^{\mathcal{U}} \circ \tau_{\alpha i}^{m_{\alpha i} m_{\beta}} \circ \pi_{\alpha i}=\tau_{\beta 0}^{m_{\alpha i} m_{\beta}} \circ i_{\alpha j, \beta 0}^{\mathcal{U}} \circ \pi_{\alpha i},
$$

and if $N_{\beta} \neq \emptyset$ then $\widetilde{\pi}_{\beta 0} \circ j_{\beta}^{* \mathcal{T}}$ is likewise.

(L6) Let $\alpha<_{\mathcal{T}} \lambda<\operatorname{lh}(\mathcal{T})$ with $\lambda$ a limit and such that:

$-(\alpha, \lambda]_{\mathcal{T}}$ does not drop in model,

- if $\alpha \in \mathscr{B}^{\mathcal{T}}$ then $\lambda \in \mathscr{B}^{\mathcal{T}}$,

- if $\alpha$ is $\mathscr{M}$-stable then $\lambda$ is $\mathscr{M}$-stable,

- if $\alpha$ is $\mathscr{N}$-stable then $\lambda$ is $\mathscr{N}$-stable.

Then:

$-(\alpha, 0)<_{\mathcal{U}}(\lambda, 0)$,

- $(\alpha, 0) \leq_{\mathcal{U}}(\beta, i) \leq_{\mathcal{U}}(\lambda, 0)$ iff $\left[i=0\right.$ and $\left.\alpha \leq_{\mathcal{T}} \beta \leq_{\mathcal{T}} \lambda\right]$,

- $i_{\alpha 0, \lambda 0}^{\mathcal{U}}\left(\mathbb{D}_{\alpha 0}, \widetilde{\mathbb{D}}_{\alpha 0}, \vec{\xi}_{\alpha 0}\right)=\left(\mathbb{D}_{\lambda 0}, \widetilde{\mathbb{D}}_{\lambda 0}, \vec{\xi}_{\lambda 0}\right)$,

- if $M_{\alpha} \neq \emptyset$ then letting $m=m_{\alpha}$ and $n=m_{\lambda}$,

$$
\pi_{\lambda 0} \circ i_{\alpha \lambda}^{\mathcal{T}}=i_{\alpha 0, \lambda 0}^{\mathcal{U}} \circ \tau_{\alpha 0}^{m n} \circ \pi_{\alpha 0}=\tau_{\lambda 0}^{m n} \circ i_{\alpha 0, \lambda 0}^{\mathcal{U}} \circ \pi_{\alpha 0},
$$

- likewise if $N_{\alpha} \neq \emptyset$.

(L7) If $\alpha \in \mathscr{B}^{\mathcal{T}}$ then $\pi_{\alpha 0}\left\lceil\rho_{\alpha}=\widetilde{\pi}_{\alpha 0}\left\lceil\rho_{\alpha} .{ }^{37}\right.\right.$

(L8) Let $\alpha<\beta<\operatorname{lh}(\mathcal{T})$ and $\alpha<\beta^{\prime}<\operatorname{lh}(\mathcal{T})$ and $\kappa \in c_{\alpha}$. Then:

- If $E_{\alpha} \in \mathbb{E}_{+}^{M_{\alpha}}$ and $0 \leq i<u_{\alpha}$ then $\tau_{\alpha i}^{m_{\alpha i} 0} \circ \pi_{\alpha i}\left\lceil\varrho_{\alpha, i+1} \subseteq \omega_{\alpha}^{*}\right.$; likewise otherwise.

- If $\pi_{\alpha \kappa}$ is defined then $\pi_{\alpha \kappa}\left\lceil\left(\kappa^{+}\right)^{M_{\alpha \kappa}} \subseteq \omega_{\alpha}^{*}\right.$.

- If $\widetilde{\pi}_{\alpha \kappa}$ is defined then $\tilde{\pi}_{\alpha \kappa}\left\lceil\left(\kappa^{+}\right)^{N_{\alpha \kappa}} \subseteq \omega_{\alpha}^{*}\right.$.

- If $\pi_{\beta 0}$ is defined then $\omega_{\alpha}^{*} \subseteq \pi_{\beta 0}$.

- If $\widetilde{\pi}_{\beta 0}$ is defined then $\omega_{\alpha}^{*} \subseteq \widetilde{\pi}_{\beta 0}$.

- If $\pi_{\beta 0}$ and $\widetilde{\pi}_{\beta^{\prime} 0}$ are both defined then they agree over $\operatorname{ex}_{\alpha}^{\mathcal{T}}$ (not just over $\left.\left(\operatorname{ex}_{\alpha}^{\mathcal{T}}\right)^{\mathrm{sq}}=\operatorname{dom}\left(\omega_{\alpha}^{*}\right)\right)$.

We write $\omega_{\alpha}$ for the restriction of $\pi_{\alpha+1,0}$ or $\tilde{\pi}_{\alpha+1,0}$ to $\operatorname{ex}_{\alpha}^{\mathcal{T}}$ (not just $\left.\left(\mathrm{ex}_{\alpha}^{\mathcal{T}}\right)^{\mathrm{sq}}\right)$, whichever is defined. Then moreover:

$-\omega_{\infty}=\bigcup_{\alpha+1<\operatorname{lh}(\mathcal{T})} \omega_{\alpha}$ is a function.

\footnotetext{
${ }^{37}$ Note then that if $\alpha+1<\operatorname{lh}(\mathcal{T})$ then for all $\kappa \in c_{\alpha} \cap \rho_{\alpha}$ such that $\left(\kappa^{+}\right)^{B_{\alpha}}<\operatorname{lh}_{\alpha}^{\mathcal{T}}$, we have $\pi_{\alpha \kappa}=\pi_{\alpha 0}$ and $\widetilde{\pi}_{\alpha \kappa}=\widetilde{\pi}_{\alpha 0}$, so $\pi_{\alpha \kappa}\left\lceil\left(\kappa^{+}\right)^{B}=\widetilde{\pi}_{\alpha \kappa}\left\lceil\left(\kappa^{+}\right)^{B_{\alpha}}\right.\right.$.
} 
(L9) The tree given by removing padding from $\mathcal{U}$ is neat. Moreover, given $\alpha+1, \beta+1<\operatorname{lh}(\mathcal{T})$ and $j \leq 2 u_{\alpha}$ and $k \leq 2 u_{\beta}$, and letting $(\alpha, j)+1$ be the successor of $(\alpha, j)$ in $\operatorname{dom}(\mathcal{U})$, we have

(a) if $(\alpha, j)<(\beta, k)$ then $\left[s_{\alpha j} \leq s_{\beta k}\right.$ and if $E_{\alpha j}^{\mathcal{U}} \neq \emptyset$ then $\left.s_{\alpha j}<s_{\beta k}\right]$,

(b) if $E_{\alpha j}^{\mathcal{U}} \neq \emptyset$ then either:

- (i) $\operatorname{cr}\left(E_{\alpha j}^{\mathcal{U}}\right)<s_{\alpha j}$, (ii) $\operatorname{str}_{\alpha j}$ is the least $M_{\alpha j}^{\mathcal{U}}$-cardinal $\geq s_{\alpha j}$, and (iii) $\operatorname{pred}^{\mathcal{U}}((\alpha, j)+1)$ is the least $(\gamma, \ell)$ such that $\operatorname{cr}\left(E_{\alpha j}^{\mathcal{U}}\right)<s_{\gamma \ell}$, or

- (i) $\operatorname{cr}\left(E_{\alpha j}^{\mathcal{U}}\right)=s_{\alpha j}$, (ii) $\operatorname{str}_{\alpha j}=\left(s_{\alpha j}^{+}\right)^{M_{\alpha j}^{\mathcal{U}}}$, (iii) $s_{\alpha j}$ is not measurable in $M_{(\alpha, j)+1}^{\mathcal{U}}$, (iv) $j$ is odd.

(L10) Let $\alpha+1<\operatorname{lh}(\mathcal{T})$ and $i<u_{\alpha}$. Then:

- If $E_{\alpha, 2 i}^{\mathcal{U}} \neq \emptyset$ then $\left[Q_{\alpha i}\right.$ is type $3, \nu\left(Q_{\alpha i}\right)$ is a limit cardinal of $Q_{\alpha i}$, and $\left.s_{\alpha, 2 i}=\nu\left(Q_{\alpha i}\right)<\mathrm{OR}^{Q_{\alpha i}}<s_{\alpha, 2 i+1}\right]$,

- if $E_{\alpha, 2 i}^{\mathcal{U}}=\emptyset$ then $s_{\alpha, 2 i}=s_{\alpha, 2 i+1}$,

- $s_{\alpha, 2 i+1}=\rho_{\omega}\left(R_{\alpha, i+1}\right)=\rho_{\omega}\left(Q_{\alpha, i+1}\right)$,

- $s_{\alpha, 2 i}, s_{\alpha, 2 i+1}$ are cardinals of $Q_{\alpha, i}, \mathfrak{C}_{\omega}\left(R_{\alpha, i+1}\right), \mathfrak{C}_{\omega}\left(Q_{\alpha, i+1}\right)$, and $Q_{\alpha, i+1}$,

- $Q_{\alpha i}\left|\rho=\mathfrak{C}_{\omega}\left(R_{\alpha, i+1}\right)\right| \rho=\mathfrak{C}_{\omega}\left(Q_{\alpha, i+1}\right)\left|\rho=Q_{\alpha, i+1}\right| \rho$ where $\rho=s_{\alpha, 2 i+1}$.

(L11) Let $\alpha<\beta<\operatorname{lh}(\mathcal{T})$ and $i \leq u_{\beta}$. Then:

- $s_{\alpha, 2 u_{\alpha}}=\nu\left(Q_{\alpha}^{\prime}\right) ;$ let $\nu=\nu\left(Q_{\alpha}^{\prime}\right),{ }^{38}$

- $Q_{\alpha}^{\prime}\left\|\nu=Q_{\alpha}^{*}\right\| \nu=\mathfrak{C}_{m_{\beta i}}\left(Q_{\beta i}\right) \| \nu,{ }^{39}$

$-\nu<\rho_{m_{\beta i}}\left(Q_{\beta i}\right)$,

- $Q_{\alpha}^{*}, Q_{\alpha}^{\prime}$ and $\mathfrak{C}_{m_{\beta i}}\left(Q_{\beta i}\right)$ agree about cardinals $<\nu$.

(L12) Let $\alpha+1<\operatorname{lh}(\mathcal{T})$ and suppose that $E_{\alpha} \in \mathbb{E}_{+}\left(M_{\alpha}\right)$. Then $E_{\alpha, 2 u_{\alpha}}^{\mathcal{U}} \neq \emptyset$ and

$$
\sup \omega_{\infty} " \nu_{\alpha}^{\mathcal{T}} \leq s_{\alpha, 2 u_{\alpha}}=\nu\left(Q_{\alpha}^{\prime}\right) \leq \omega_{\infty}\left(\nu_{\alpha}^{\mathcal{T}}\right) .
$$

Now suppose also that $u_{\alpha}>0$. Let $\bar{\nu}=\sup _{\beta<\alpha} \nu_{\beta}^{\mathcal{T}}$. Note that

$$
\bar{\nu} \leq \varrho_{\alpha 1}<\varrho_{\alpha 2}<\ldots<\varrho_{\alpha u_{\alpha}} \leq \nu_{\alpha}^{\mathcal{T}} .
$$

(Here if $u_{\alpha} \leq 1$ and $\alpha$ is a limit we could have $\bar{\nu}=\nu_{\alpha}^{\mathcal{T}}$.) Let $i<u_{\alpha}$ and let $j \in\{2 i, 2 i+1\}$. If $E_{\alpha j}^{\mathcal{U}} \neq \emptyset$ then

$$
\sup \omega_{\infty} " \varrho_{\alpha, i+1} \leq s_{\alpha j} \leq \omega_{\infty}\left(\varrho_{\alpha, i+1}\right) .
$$

\footnotetext{
${ }^{38}$ Recall that if $\omega_{\alpha}^{*}$ is non- $\nu$-low then $Q_{\alpha}^{\prime}=Q_{\alpha}^{*}$ and if $\omega_{\alpha}^{*}$ is $\nu$-low and $\nu^{\prime}=\psi_{\omega_{\alpha}^{*}}\left(\nu_{\alpha}^{\mathcal{T}}\right)$ then

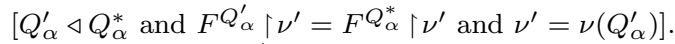

39 If $\nu=\left(\gamma^{+}\right)^{Q_{\alpha}^{*}}$ then it seems possible that $N=\mathfrak{C}_{m_{\alpha+1}}\left(Q_{\alpha+1,0}\right) \mid \nu$ is active with an extender $G$, in which case of course $Q_{\alpha}^{*} \mid \nu \neq N$, but $Q_{\alpha}^{*}\left\|\operatorname{OR}\left(Q_{\alpha}^{*}\right)=\operatorname{Ult}(N, G)\right\| \operatorname{OR}\left(Q_{\alpha}^{*}\right)$.
} 
We now begin the construction. Recall that $C=\left(\rho^{C}, M, N\right)$ was defined in the proof of Claim 1 of 6.18 , as were $\mathbb{C}, \lambda^{\mathbb{C}}, M^{\prime}=S_{\lambda^{\mathbb{C}}}^{\mathbb{C}}, M=\mathfrak{C}_{\omega}\left(M^{\prime}\right)$, and likewise $\widetilde{\mathbb{C}}$ etc. We have $\operatorname{deg}^{\mathcal{T}}(0)=\left(m_{0}, n_{0}\right)=$ the degree of $C$; that is, either

- $m_{0}=0$ and $M$ is type 3 with $\nu(M)=\rho^{C}$, or

$-m_{0} \geq 0$ and $\rho_{m_{0}+1}^{M} \leq \rho^{C}<\rho_{m_{0}}^{M}$

and likewise for $n_{0}, N$. We have $M_{0}=M$ and $N_{0}=N$. Let $\mathbb{D}_{00}=\mathbb{C}$ and $\xi_{00}=\lambda^{\mathbb{C}}$ and

$$
\pi_{00}: \mathfrak{C}_{0}\left(M_{0}\right) \rightarrow \mathfrak{C}_{m_{0}}\left(Q_{00}\right)
$$

be the core embedding. (Recall that $M, N$ are fully sound, but $M$ may or may not be $\mathbb{C}$-standard, and $Q_{00}=S_{\lambda \mathbb{C}}^{\mathbb{C}}$ may or may not be sound, and likewise $N, \mathbb{D}$; in the notation that assumes $1<\ln (\mathcal{T})$, the core embedding is $\tau_{00}^{\omega m_{0}}$.) We define $\widetilde{\mathbb{D}}_{00}, \widetilde{\xi}_{00}, \widetilde{\pi}_{00}$ analogously. Then $\pi_{00} \uparrow \rho_{0}=\mathrm{id}=\widetilde{\pi}_{00} \uparrow \rho_{0}$, which gives inductive hypothesis $(\mathrm{L} 7)$ (for $(\mathcal{T} \uparrow 1, \mathcal{U} \uparrow(0,1))$ ), and the others are trivial as $\ln (\mathcal{T} \uparrow 1)=1=\ln (\mathcal{U} \uparrow(0,1))$

Now let $\lambda$ be a limit ordinal and suppose that the inductive hypotheses hold of $\mathcal{T} \uparrow \lambda$ and $\mathcal{U} \uparrow(\lambda, 0)$; we will define $\mathcal{U} \uparrow(\lambda, 1)$ and $\mathcal{T} \uparrow(\lambda+1)$ and verify that the hypotheses still hold. Note that $\mathcal{U} \uparrow(\lambda, 0)$ has limit length and is cofinally non-padded. Let $c=\Sigma_{V}(\mathcal{U} \uparrow(\lambda, 0))$.

We can define $\Sigma_{M}(\mathcal{T} \uparrow \lambda)$ as the unique branch $b$ such that for eventually all $\alpha \in b$, we have $(\alpha, 0) \in c$. For by inductive hypotheses (L5)a-(L5)c, there are only boundedly many $(\beta, k) \in c$ with $k>0$, so by hypothesis (L4), this branch is well-defined and $\mathcal{T}$-cofinal. Similarly, there are only finitely many drops in model along $b$, and there are unique choices for $\pi_{\lambda 0}$, etc, maintaining the inductive hypotheses.

We now move to the successor case. Suppose that the inductive hypotheses hold for $\mathcal{T}\lceil\delta+1$ and $\mathcal{U} \uparrow(\delta, 1)$. We will define $\mathcal{U} \uparrow(\delta+1,1)$ and show that they hold for $\mathcal{T}\lceil\delta+2$ and $\mathcal{U} \uparrow(\delta+1,1)$. Note that this step involves the use of just one extender in $\mathcal{T}$, and finitely many in $\mathcal{U}$.

CASE 1. $u_{\delta}=0$ and $M_{\delta} \neq \emptyset$ and $E_{\delta} \in \mathbb{E}_{+}^{M_{\delta}}\left(\right.$ so $\left.E_{\delta}=F\left(M_{\delta}\right)\right)$.

SubClaim 5.1. $\xi_{\delta 0}$ is $\mathbb{D}_{\delta 0}$-standard.

Proof. Suppose otherwise. Then by induction with (L2), $\delta$ is $\mathscr{M}$-stable.

Now $\delta \in \mathscr{B}^{\mathcal{T}}$. For suppose $\delta \in \mathscr{M}^{\mathcal{T}}$ and let $\beta=\max \left(\mathscr{B}^{\mathcal{T}} \cap[0, \delta]_{\mathcal{T}}\right)$. Then

$$
\rho\left(B_{\beta}\right) \leq \operatorname{cr}\left(i_{\beta \delta}^{\mathcal{T}}\right)<\rho_{0}\left(M_{\beta}\right),
$$

so $\rho^{C}<\rho_{0}^{M}$. But then $\vartheta_{\delta}=i_{0 \delta}^{\mathcal{T}}\left(\rho^{C}\right)<\rho_{0}\left(M_{\delta}\right)$, and

$$
\left(\vartheta_{\delta}^{+}\right)^{M_{\delta}} \leq \mathrm{OR}\left(M_{\delta}\right)=\operatorname{lh}\left(E_{\delta}\right),
$$

so $u_{\delta}>0$, by 6.22 , contradiction.

So $\delta \in \mathscr{B}^{\mathcal{T}}$. Note then that by 6.22 and since $u_{\delta}=0$, we have $\vartheta_{\delta} \geq \mathrm{OR}^{M_{\delta}}$, so $M$ is type 3 with $\rho^{C}=\nu(M)$. But then by $6.9, \lambda^{\mathbb{C}}$ is $\mathbb{C}$-standard, so by (L1), $\xi_{\delta 0}$ is $\mathbb{D}_{\delta 0^{-}}$-standard. This completes the proof of the subclaim. 
Let $\lambda=\sup _{\beta<\delta} \operatorname{lh}_{\beta}^{\mathcal{T}}$. Then $\lambda \leq \rho_{m_{\delta}}\left(M_{\delta}\right)$, so $\pi_{\delta 0} " \lambda \subseteq \rho_{m_{\delta}}\left(Q_{\delta 0}\right)$, but $\tau_{\delta 0}^{m_{\delta} 0} \uparrow$ $\rho_{m_{\delta}}\left(Q_{\delta 0}\right)=\mathrm{id}$, so

$$
\omega_{\delta}^{*}\left\lceil\lambda=\pi_{\delta 0} \uparrow \lambda\right.
$$

If $\omega_{\delta}^{*}$ is non- $\nu$-low then set $E_{\delta 0}^{\mathcal{U}}=$ the $*$-least extender $E^{*} \in M_{\delta 0}^{\mathcal{U}}$ witnessing $6.3(3 \mathrm{~b})$ for $\left(\mathbb{D}_{\delta 0}, Q_{\delta 0}\right)$; and as required by $(\mathrm{L} 11)$, set $s_{\delta 0}=\nu\left(Q_{\delta 0}\right)$ in this case. Suppose now that $\omega_{\delta}^{*}$ is $\nu$-low. In particular, $E_{\delta}$ is type 3 . Let $\nu^{\prime}=\psi_{\omega_{\delta}^{*}}\left(\nu_{\delta}^{\mathcal{T}}\right)$ and $Q^{\prime} \triangleleft Q_{\delta 0}$ be as in 1.1. By 6.7 and 6.9, and as $\nu^{\prime}$ is a $Q_{\delta 0}$-cardinal, we get $Q^{\prime}=S_{\gamma}^{\mathbb{D}_{\delta 0}}$ for some $\mathbb{D}_{\delta 0}$-standard $\gamma$. Let $E_{\delta 0}^{\mathcal{U}}$ be the $*$-least $E^{*} \in M_{\delta 0}^{\mathcal{U}}$ witnessing $6.3(3 \mathrm{~b})$ for $Q^{\prime}$, and $s_{\delta 0}=\nu^{Q^{\prime}}$. Note that in both cases, by $*$-minimality, $\operatorname{str}_{\delta 0}$ is the least $M_{\delta 0}^{\mathcal{U}}$-cardinal $\rho \geq s_{\delta 0}$.

Let $\kappa=\operatorname{cr}_{\delta}^{\mathcal{T}}$ and $\alpha=\operatorname{pred}^{\mathcal{T}}(\delta+1)$ and $i=i_{\alpha \kappa}$. Note that $M_{\delta+1}^{*}=M_{\alpha \kappa}$ and $m_{\alpha \kappa}=m_{\delta+1}$ and $N_{\delta+1}^{*}=N_{\alpha \kappa}$ and $n_{\alpha \kappa}=n_{\delta+1}$ (with each of these equalities, it is included that the object on the left is defined iff the one on the right is). We can and do set $\operatorname{pred}^{\mathcal{U}}(\delta+1,0)=(\alpha, 2 i)$, by properties (L9)-(L12). The identities of $\mathbb{D}_{\delta+1,0}, \widetilde{\mathbb{D}}_{\delta+1,0}, \xi_{\delta+1,0}, \widetilde{\xi}_{\delta+1,0}$ are determined by property (L5)d. We define $\pi_{\delta+1,0}$ and/or $\tilde{\pi}_{\delta+1,0}$ as usual.

It is straightforward and mostly routine to show that the inductive hypotheses are maintained, and we leave this mostly to the reader, just making a couple of remarks. Consider the verification of (L2) at $\delta+1$ in the case that $M_{\delta+1} \neq \emptyset$ and $[0, \delta+1]_{\mathcal{T}}$ does not drop, and $\alpha$ is $\mathscr{M}$-stable, but $\delta+1$ is not. So $\vartheta_{\alpha} \leq \operatorname{cr}\left(E_{\delta}\right)$ and note that $E_{\alpha} \in \mathbb{E}_{+}\left(M_{\alpha}\right), \vartheta_{\alpha}<\nu\left(E_{\alpha}\right)$, and $\left(\vartheta_{\alpha}^{+}\right)^{M_{\alpha}} \leq \operatorname{lh}\left(E_{\alpha}\right)$. So by $6.22, u_{\alpha}>0$, and since $\vartheta_{\alpha} \leq \operatorname{cr}\left(E_{\delta}\right)$ but $E_{\delta}$ is $M_{\alpha}$-total, (L4)b implies $\operatorname{pred}^{\mathcal{U}}(\delta+1,0)=(\alpha, 2)$, and by (L3), $\xi_{\alpha 2}$ is $\mathbb{D}_{\alpha 2}$-standard. Considering the definition of $\left(\mathbb{D}_{\delta+1,0}, \xi_{\delta+1,0}\right)$ given by (L5)d, this yields (L2) (in the case under consideration). Hypothesis (L11) is pretty standard, but we remark that if $\operatorname{ex}_{\delta}^{\mathcal{T}}$ is type 1 or 3 (hence $Q_{\delta}^{\prime}$ has the same type) and $\nu^{\prime}=\nu\left(F\left(Q_{\delta}^{\prime}\right)\right)=\left(\gamma^{+}\right)^{Q_{\delta}^{\prime}}$ (some $\gamma)$, then we can only expect $Q_{\delta}^{\prime}\left\|\nu^{\prime}=Q_{\delta+1,0}\right\| \nu^{\prime}$, because $6.3(3 \mathrm{~b})$ only gives that $F\left(Q_{\delta}^{\prime}\right)\left\lceil\nu^{\prime} \subseteq E_{\delta}^{\mathcal{U}}\left\lceil\nu^{\prime}\right.\right.$, so while $Q_{\delta}^{\prime} \mid \nu^{\prime}$ is passive, it might be that $Q_{\delta+1,0} \mid \nu^{\prime}$ is active with an extender $F^{\prime}$. ${ }^{40}$ Regarding (L8), by line (13), and because $\omega_{\delta}^{*} \subseteq \pi_{\delta+1,0}$ and/or $\omega_{\delta}^{*} \subseteq \tilde{\pi}_{\delta+1,0}$, we have maintained the well-definedness of $\omega_{\infty}$. And regarding (L12), the fact, for example if $\pi_{\delta+1,0}$ is defined, that

$$
\omega_{\infty}\left(\nu_{\delta}^{\mathcal{T}}\right)=\pi_{\delta+1,0}\left(\nu_{\delta}^{\mathcal{T}}\right) \geq s_{\delta 0}
$$

follows from our choice of $E^{*}$ (this is why we use $Q^{\prime}$ when $\omega_{\delta}^{*}$ is $\nu$-low).

CASE 2. $u_{\delta}=0$ and $E_{\delta} \notin \mathbb{E}_{+}^{M_{\delta}}$ (so $E_{\delta}=F\left(N_{\delta}\right)$ ).

By symmetry with the previous case.

CASE 3. $u_{\delta}>0$ and $E_{\delta} \in \mathbb{E}_{+}^{M_{\delta}}$ and if $\delta$ is $\mathscr{M}$-stable and $\vartheta_{\delta}<\mathrm{OR}^{M_{\delta}}$ then $\operatorname{lh}\left(E_{\delta}\right)<\left(\vartheta_{\delta}^{+}\right)^{M_{\delta}}$.

\footnotetext{
${ }^{40}$ If $F^{\prime}=\emptyset$, then standard arguments with condensation show that $Q_{\delta+1,0} \| \lambda^{\prime}=$ $Q_{\delta}^{\prime} \| \lambda^{\prime}$, where $\lambda^{\prime}=\operatorname{OR}\left(Q_{\delta}^{\prime}\right)$; if $F^{\prime} \neq \emptyset$, standard arguments show that $Q_{\delta+1,0} \| \lambda^{\prime}=$ $\operatorname{Ult}\left(Q_{\delta+1,0} \| \lambda^{\prime}, F^{\prime}\right) \| \lambda^{\prime}$. Analogously (and irrespective of the type of $\operatorname{ex}_{\delta}^{\mathcal{T}}$ ), if $Q_{\delta+1,0}^{\prime} \mid \nu^{\prime}$ is passive, it seems that $Q_{\delta+1,0}^{\prime} \mid \lambda^{\prime}$ might be active.
} 
Let $\varrho=\varrho_{\delta 1}$; then $\varrho$ is a cardinal of $M_{\delta}$, so $\varrho \leq \rho_{0}\left(M_{\delta}\right)$ and if $\delta$ is $\mathscr{M}$-stable then $\varrho \leq \vartheta_{\delta}$. We will first determine $\mathcal{U} \uparrow((\delta, 2)+1)$ and the associated objects (that is, through $M_{\delta 2}^{\mathcal{U}}, \mathbb{D}_{\delta 2}, \xi_{\delta 2}, Q_{\delta 1}, \pi_{\delta 1}$, etc); this splits into various subcases. SuBCASE 3.1. $\varrho<\rho_{0}\left(M_{\delta}\right)$.

Set $E_{\delta 0}^{\mathcal{U}}=\emptyset$; so $\operatorname{pred}^{\mathcal{U}}(\delta, 1)=(\delta, 0)$ and $M_{\delta 1}^{\mathcal{U}}=M_{\delta 0}^{\mathcal{U}}$ and $i_{\delta 0, \delta 1}^{\mathcal{U}}=$ id. Set $\mathbb{D}_{\delta 1}=\mathbb{D}_{\delta 0}$. Let $\varphi: \mathfrak{C}_{0}\left(M_{\delta}\right) \rightarrow \mathfrak{C}_{0}\left(Q_{\delta 0}\right)$ be $\varphi=\tau_{\delta 0}^{m_{\delta} 0} \circ \pi_{\delta 0}$. Let $R=\varphi\left(M_{\delta 1}\right)$. Set $s_{\delta 0}=s_{\delta 1}=\varphi(\varrho)=\rho_{\omega}^{R}$. Note $\varphi(\varrho)$ is a cardinal of $Q_{\delta 0}\left(\right.$ as $\pi_{\delta 0}$ and $\tau_{\delta 0}^{m_{\delta} 0}$ are c-preserving). Moreover, if $\xi_{\delta 0}$ is non- $\mathbb{D}_{\delta 0}$-standard then $\varphi(\varrho) \leq \rho_{\omega}\left(Q_{\delta 0}\right)$; for by non-standardness, (L1) and (L2), $\delta$ is $\mathscr{M}$-stable and $\varphi=\pi_{\delta 0}$, and since $\varrho \leq \vartheta_{\delta}$, therefore $\varphi(\varrho) \leq \pi_{\delta 0}\left(\vartheta_{\delta}\right)=\rho_{\omega}^{Q_{\delta 0}}$. So we can fix $\xi=\xi_{\delta 1}$ with $\mathfrak{C}_{0}(R)=\mathfrak{C}_{\omega}\left(S_{\xi}^{\mathbb{D}} \boldsymbol{D}_{\delta 1}\right)$. So $R_{\delta 1}=S_{\xi_{\delta 1}}^{\mathbb{D}_{\delta 1}}=S_{\xi}^{\mathbb{D}_{\delta 0}}$. We set

$$
\pi_{\delta 1}=\varphi\left\lceil\mathfrak{C}_{0}\left(M_{\delta 1}\right): \mathfrak{C}_{0}\left(M_{\delta 1}\right) \rightarrow \mathfrak{C}_{0}(R)=\mathfrak{C}_{\omega}\left(R_{\delta 1}\right) .\right.
$$

We now define $E_{\delta 1}^{\mathcal{U}}, M_{\delta 2}^{\mathcal{U}}, \mathbb{D}_{\delta 2}, \xi_{\delta 2}$, and hence $Q_{\delta 1}=S_{\xi_{\delta 2}}^{\mathbb{D}_{\delta 2}}$. Recall we want $\xi_{\delta 2}$ to be $\mathbb{D}_{\delta 2}$-standard and $\mathfrak{C}_{\omega}\left(Q_{\delta 1}\right)=\mathfrak{C}_{\omega}\left(R_{\delta 1}\right)$.

Now if $\xi_{\delta 1}$ is $\mathbb{D}_{\delta 1}$-standard we set $E_{\delta 1}^{\mathcal{U}}=\emptyset$ (so $\operatorname{pred}^{\mathcal{U}}(\delta, 2)=(\delta, 1)$ etc), $\mathbb{D}_{\delta 2}=\mathbb{D}_{\delta 1}$ and $\xi_{\delta 2}=\xi_{\delta 1}$.

Suppose that $\xi_{\delta 1}$ is non- $\mathbb{D}_{\delta 1}$-standard. So $R_{\delta 1}$ is sound. Let $E_{\delta 1}^{\mathcal{U}}$ be the *-least $G \in M_{\delta 1}$ which is a $\mathbb{D}_{\delta 1}$-nice witness for $R_{\delta 1}$. Let $\mu=\operatorname{cr}(G)$. (Note we can) let $(\alpha, j)=\operatorname{pred}^{\mathcal{U}}(\delta, 2)$ be least $\leq(\delta, 1)$ such that $\mu<s_{\alpha j}$, if such exists; otherwise $\mu=s_{\delta 1}$ and $(\alpha, j)=(\delta, 2)$. If $E_{\alpha} \in \mathbb{E}_{+}\left(M_{\alpha}\right)$ then let $\mathbb{F}=\mathbb{D}_{\alpha j}$ and $\zeta=\xi_{\alpha j}$, and if $2 i=j$ then let $P=Q_{\alpha i}$, and if $2 i-1=j$ then $P=R_{\alpha i}$. Otherwise let $\mathbb{F}=\widetilde{\mathbb{D}}_{\alpha j}, \zeta=\widetilde{\xi}_{\alpha j}$ and $P=\widetilde{Q}_{\alpha i}$ or $P=\widetilde{R}_{\alpha i}$. Let $f=i_{\alpha j, \delta 2}^{\mathcal{U}}$.

By properties (L10),(L11), $(P \sim R) \mid \mu$, and note that $P \mid \mu=S_{\gamma}^{\mathbb{F}}$ for some $\mathbb{F}$-standard $\gamma<\zeta$. Since $G$ is a nice witness, $f(\mu)>\varphi(\varrho)$, so $R_{\delta 1} \triangleleft f(P \mid \mu)$, and note that $\varphi(\varrho)$ is a cardinal of $f(P \mid \mu)$. We set $\mathbb{D}_{\delta 2}=f(\mathbb{F})$, and let $\xi_{\delta 2}$ be the $\xi<f(\zeta)$ such that $\mathfrak{C}_{0}(R)=\mathfrak{C}_{\omega}\left(S_{\xi}^{\mathbb{D} \delta 2}\right)$. Because $G$ is a nice witness, the agreement between $M_{\delta 2}^{\mathcal{U}}$ and $\operatorname{Ult}\left(M_{\delta 1}^{\mathcal{U}}, G\right)$ implies that $\xi_{\delta 2}$ is strongly $\mathbb{D}_{\delta 2^{-}}$ standard (note that this only depends on $S_{\xi_{\delta 2}}^{\mathbb{D}_{\delta 2}}$ in $M_{\delta 2}^{\mathcal{U}}$ or in $\operatorname{Ult}\left(M_{\delta 1}^{\mathcal{U}}, G\right)$, not on the relevant constructions themselves).

SubCASE 3.2. $\varrho=\rho_{0}\left(M_{\delta}\right)$.

So $M_{\delta}$ is active type 3 . Let $E=F^{M_{\delta}}$ and $Q=Q_{\delta 0}$ and $F=F^{Q}$ and $\nu=\nu(F)$. Let $v: \mathfrak{C}_{0}\left(M_{\delta}\right) \rightarrow \mathfrak{C}_{0}(Q)$ be $v=\tau_{\delta 0}^{m_{\delta} 0} \circ \pi_{\delta 0}$. Let $\psi=\psi_{v}$.

SubsubCASE 3.2.1. $v$ is non- $\nu$-high; that is, $\psi(\varrho) \leq \nu$.

Proceed as in Subcase 3.1, but using $\varphi=\psi$ instead.

SubsubCase 3.2.2. $v$ is $\nu$-high; that is, $\psi(\varrho)>\nu$.

Here we proceed basically as in [10]. Let $E_{\delta 0}^{\mathcal{U}}$ be the $*$-least witness to $6.3(3 \mathrm{~b})$ for $\left(\mathbb{D}_{\delta 0}, Q\right)$ and set $s_{\delta 0}=\nu$. Let $\mathcal{T}^{\prime}$ be the putative iteration tree on $C$ of the form $\left(\mathcal{T}\lceil\delta+1)^{\wedge} E\right.$. Then $M_{\delta 1} \triangleleft \mathfrak{C}_{0}\left(M_{\delta+1}^{\mathcal{T}^{\prime}}\right)$. Let $\alpha=\operatorname{pred}^{\mathcal{T}^{\prime}}(\delta+1)$ and $\kappa=\operatorname{cr}(E)$ and $i=i_{\alpha \kappa}$. We set $\operatorname{pred}^{\mathcal{U}}(\delta, 1)=(\alpha, 2 i)$; as in Case 1 this works. Let $\mathbb{F}, \zeta, P, f$ be defined from $(\alpha, 2 i)$ as in Subcase 3.1. For notational specificity, let us assume that $E_{\alpha} \in \mathbb{E}_{+}\left(M_{\alpha}\right)$; the other case is likewise, but instead of $P=Q_{\alpha i}$, we have 
$P=\widetilde{Q}_{\alpha i}$, etc. So $P=Q_{\alpha i}=S_{\zeta}^{\mathbb{D}_{\alpha, 2 i}}$ (as $j=2 i$ ). We have $M_{\alpha \kappa}=M_{\alpha i}$ and $\kappa<\rho_{m_{\alpha \kappa}}\left(M_{\alpha \kappa}\right)$ and $\kappa<\rho_{0}\left(M_{\delta}\right)$,

$$
\pi_{\alpha \kappa}: \mathfrak{C}_{0}\left(M_{\alpha \kappa}\right) \rightarrow \mathfrak{C}_{m_{\alpha \kappa}}(P) \text { and } v: \mathfrak{C}_{0}\left(M_{\delta}\right) \rightarrow \mathfrak{C}_{0}(Q) .
$$

Let $\chi=\left(\kappa^{+}\right)^{M_{\delta}}=\left(\kappa^{+}\right)^{M_{\alpha \kappa}}$. We have (and let) $\bar{M}=M_{\alpha \kappa}|| \chi=M_{\delta} \mid \chi$. We have $\pi_{\alpha \kappa}\left\lceil\chi=v\left\lceil\chi=\omega_{\infty}\left\lceil\chi\right.\right.\right.$. Let $\kappa^{\prime}=\pi_{\alpha \kappa}(\kappa)=v(\kappa)=\operatorname{cr}(F)$ and

$$
\chi^{\prime}=\sup \pi_{\alpha \kappa} " \chi=\sup v " \chi=\sup \omega_{\infty} " \chi .
$$

Then $\kappa^{\prime}<\rho_{m_{\alpha \kappa}}(P)$ is a cardinal of $P$ and $Q$ and (letting) $\bar{P}=P\left\|\chi^{\prime}=Q\right\| \chi^{\prime}$, then $\bar{P}$ has largest cardinal $\kappa^{\prime}$, and there is $\gamma \leq \zeta$ which is $\mathbb{D}_{\alpha, 2 i}$-standard with $\bar{P}=S_{\gamma}^{\mathbb{D}_{\alpha, 2 i}}$. Let $\bar{\pi}: \bar{M} \rightarrow \bar{P}$ be $\bar{\pi}=\pi_{\alpha \kappa}\left\lceil\bar{M}\right.$. So $\bar{\pi}$ is cofinal $\Sigma_{1}$-elementary. Let

$$
\bar{U}=\operatorname{Ult}_{0}(\bar{M}, E)=\operatorname{Ult}_{0}(\bar{M}, E\lceil\nu(E))
$$

and $\bar{\psi}: \bar{U} \rightarrow \operatorname{Ult}_{0}(\bar{P}, F\lceil\nu)$ be induced from $\bar{\pi}$ and $v$ via the Shift Lemma. 41 So $\bar{\psi}$ is cofinal $\Sigma_{1}$-elementary and $\bar{\psi} \circ i_{E}^{\bar{M}}=i_{F \mid \nu}^{\bar{P}} \circ \bar{\pi}$ and $v \subseteq \bar{\psi}$ (and note $\operatorname{rg}(v) \subseteq Q \mid \nu)$. We have $\bar{P} \in M_{\alpha, 2 i}^{\mathcal{U}}$. Let $\psi^{\prime}: \operatorname{Ult}_{0}(\bar{P}, F \uparrow \nu) \rightarrow f(\bar{P})$ be the natural factor map; that is, for $g \in \bar{P}$ and generators $a \in \nu^{<\omega}$,

$$
\psi^{\prime}\left([a, g]_{F \mid \nu}^{\bar{P}}\right)=[a, g]_{E_{\delta 0}^{\mathcal{U}}}^{M_{\mathcal{U}}^{\mathcal{U}}}
$$

(recalling that $F\left\lceil\nu \subseteq E_{\delta 0}^{\mathcal{U}}\right.$ ). So $\psi^{\prime}$ is $\Sigma_{0}$-elementary and c-preserving and $\psi^{\prime} \circ i_{F \mid \nu}^{\bar{P}}=f\left\lceil\bar{P}\right.$ and $\operatorname{cr}\left(\psi^{\prime}\right) \geq \nu$. Let $\psi_{1}=\psi^{\prime} \circ \bar{\psi}$. So $\psi_{1}: \bar{U} \rightarrow f(\bar{P})$, and $\psi_{1}$ is also $\Sigma_{0}$-elementary c-preserving, commutes, and $v \subseteq \psi_{1}$, and of course $M_{\delta} \| \mathrm{OR}^{M_{\delta}} \unlhd \bar{U}$ and $\varrho=\nu(E)$ is a cardinal of $\bar{U}$.

SubClaim 5.2. $\psi_{1}(\varrho)>\nu=\nu(F)=s_{\delta 0}$.

Proof. We have $\nu=\nu(F)=s_{\delta 0}$ by definition. If $\chi^{\prime}=\left(\left(\kappa^{\prime}\right)^{+}\right)^{Q}$ then in fact $\bar{\psi}(\varrho)>\nu$, because in fact $\bar{\psi} \subseteq \psi=\psi_{v}$, and by subsubcase hypothesis, $\psi(\varrho)>\nu$. So suppose $\chi^{\prime}<\left(\left(\kappa^{\prime}\right)^{+}\right)^{Q}$. Let $\bar{P}^{+}=Q \mid\left(\left(\kappa^{\prime}\right)^{+}\right)^{Q}$, so $\bar{P}=\left(\bar{P}^{+}\right) \| \chi^{\prime}$, so noting that $\bar{P}^{+} \in M_{\alpha, 2 i}^{\mathcal{U}}$, we have $f(\bar{P})=\left(f\left(\bar{P}^{+}\right)\right) \| f\left(\chi^{\prime}\right)$. Let $\psi_{1}^{+}: \bar{U} \rightarrow f\left(\bar{P}^{+}\right)$be $\psi_{1}^{+}=$inc $\circ \psi_{1}$, where inc denotes inclusion. Then $\psi_{1}^{+}$is also $\Sigma_{0}$-elementary c-preserving, and $\psi_{1}, \psi_{1}^{+}$have the same graph. But $\psi_{1}^{+}=\sigma \circ(\psi\lceil\bar{U})$, where

$$
\sigma: \operatorname{Ult}_{0}\left(\bar{P}^{+}, F\right) \rightarrow f\left(\bar{P}^{+}\right)
$$

is the natural factor map. Since $\psi(\varrho)>\nu$ by subsubcase hypothesis, therefore $\psi_{1}^{+}(\varrho)>\nu$, as desired.

Let $\mathbb{D}_{\delta 1}=f(\mathbb{F})$ and $R=\psi_{1}\left(M_{\delta 1}\right)$. Then $R \triangleleft f(\bar{P})$ and $R=S_{\xi}^{\mathbb{D}_{\delta 1}}$ for some $\xi<f(\gamma)$; let $\xi_{\delta 1}$ be this $\xi$. Let $\pi_{\delta 1}=\psi_{1}\left\lceil\mathfrak{C}_{0}\left(M_{\delta 1}\right)\right.$ (a fully elementary map).

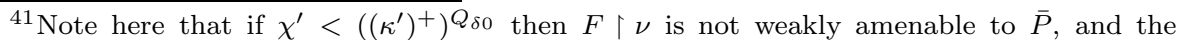
ultrapower would be different if we used the full $F$ (with generators through $\mathrm{OR}^{Q}$ ), and actually if $F$ is of supertstrong type, it is not clear how it should even be defined.
} 


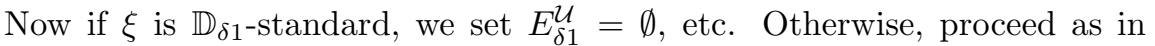
Subcase 3.1 to define $E_{\delta 1}^{\mathcal{U}}$ etc. In either case, $s_{\delta 0}=\nu<\psi_{1}(\varrho)=s_{\delta 1}$, by Subclaim 5.2.

This completes the definition of $\mathcal{U} \uparrow(\delta, 2)$ in all subcases. We now proceed in general for Case 3 , to determine $\mathcal{U} \uparrow(\delta+1,1)$ etc. If $u_{\delta}=1$ then we have already determined

$$
\omega_{\delta}^{*}=\pi_{\delta}^{*}=\tau_{\delta 1}^{\omega 0} \circ \pi_{\delta 1}: \operatorname{ex}_{\delta}^{\mathcal{T}} \rightarrow Q_{\delta}^{*}=Q_{\delta 1} .
$$

Here $\omega_{\delta}^{*}$ is $\Sigma_{1}$-elementary and so non- $\nu$-low, by 1.1. So $Q_{\delta}^{\prime}=Q_{\delta}^{*}$ and (following (L11)) we set $E_{\delta 2}^{\mathcal{U}}$ be the *-least background for $Q_{\delta}^{*}$, and $s_{\delta 2}=\nu\left(F\left(Q_{\delta}^{*}\right)\right)$.

Now $s_{\delta 1}=\rho_{\omega}\left(R_{\delta 1}\right)=\rho_{\omega}\left(Q_{\delta 1}\right) \leq s_{\delta 2}$. We claim that if $E_{\delta 1}^{\mathcal{U}} \neq \emptyset$ then $s_{\delta 1}<s_{\delta 2}$. For otherwise $s_{\delta 1}=s_{\delta 2}$, which implies $R_{\delta 1}=Q_{\delta 1}$ is type 1 or type 3 , but then by $6.9, \xi_{\delta 1}$ is $\mathbb{D}_{\delta 1}$-standard, so $E_{\delta 1}^{\mathcal{U}}=\emptyset$, contradiction. (Also if $E_{\delta 0}^{\mathcal{U}} \neq \emptyset=E_{\delta 1}^{\mathcal{U}}$, then $s_{\delta 0}<\psi_{1}(\varrho)=s_{\delta 1} \leq s_{\delta 2}$, by Subclaim 5.2.) The remaining definitions and propagation of inductive hypotheses are like in Case 1.

If $u_{\delta}>1$ then we next repeat the preceding subcases, working with $M_{\delta 2}$, $\pi_{\delta 1}$, etc, in place of $M_{\delta 1}, \pi_{\delta 0}$, etc. We iterate this until producing $\omega_{\delta}^{*}, Q_{\delta}^{*}$ and $E_{\delta, 2 u_{\delta}}^{\mathcal{U}}$ (as above, $\omega_{\delta}^{*}$ is non- $\nu$-low). This completes the definition of $\mathcal{U} \uparrow(\delta+1,1)$.

CASE 4. $u_{\delta}>0$ and $E_{\delta} \notin \mathbb{E}_{+}^{M_{\delta}}$ and if $\delta$ is $\mathscr{N}$-stable and $\vartheta_{\delta}<\mathrm{OR}^{N_{\delta}}$ then $\operatorname{lh}\left(E_{\delta}\right)<\left(\vartheta_{\delta}^{+}\right)^{N_{\delta}}$.

By symmetry.

CASE 5. $u_{\delta}>0$ and $E_{\delta} \in \mathbb{E}_{+}^{M_{\delta}}$ and $\delta$ is $\mathscr{M}$-stable and $\vartheta_{\delta}<\mathrm{OR}^{M_{\delta}}$ and $\left(\vartheta_{\delta}^{+}\right)^{M_{\delta}} \leq$ $\operatorname{lh}\left(E_{\delta}\right)$.

This case proceeds mostly like the preceding cases, but the first step is a little different. Recall that here the reversed $\left(\mathcal{T}, \delta, \operatorname{lh}\left(E_{\delta}\right)\right)$-dropdown sequence begins with $\left(M_{\delta}, 0\right),\left(M_{\delta}, \vartheta_{\delta}\right)$, and since $\delta$ is $\mathscr{M}$-stable, $\left(\mathbb{D}_{\delta 0}, \xi_{\delta 0}\right)=i_{00, \delta 0}^{\mathcal{U}}\left(\mathbb{C}, \lambda^{\mathbb{C}}\right)$, and recall that $m_{0}<\omega$ and $M$ is fully sound and either

- $m_{0}=0$ and $M=S_{\lambda^{\mathbb{C}}}^{\mathbb{C}}$ is type 3 with $\nu(M)=\rho^{C}$, or

$-M=\mathfrak{C}_{m_{0}+1}\left(S_{\lambda^{\mathbb{C}}}^{\mathbb{C}}\right)$ is fully sound with $\rho_{m_{0}+1}^{M}=\rho^{C}<\rho_{m_{0}}^{M}$.

We set $E_{\delta 0}^{\mathcal{U}}=\emptyset$ and $\mathbb{D}_{\delta 1}=\mathbb{D}_{\delta 0}, \xi_{\delta 1}=\xi_{\delta 0}, m_{\delta 1}=m_{\delta 0}=m_{\delta}=m_{0}$, etc, so $R_{\delta 1}=Q_{\delta 0}$. (We also set $\widetilde{\xi}_{\delta 1}=\uparrow$.) We will set

$$
s_{\delta 0}=s_{\delta 1}=\rho_{\omega}^{R_{\delta 1}}=i_{00, \delta 0}^{\mathcal{U}}\left(\rho^{C}\right)=i_{00, \delta 1}^{\mathcal{U}}\left(\rho^{C}\right)=\psi_{\pi_{\delta 0}}\left(\vartheta_{\delta}\right) .
$$

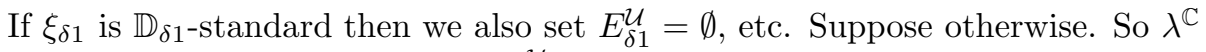
is non-C-standard, $R_{\delta 1}=Q_{\delta 0}=i_{00, \delta 1}^{\mathcal{U}}(M)$, and these models are $\omega$-sound. So $\rho^{C}<\rho_{0}^{M}$ (by 6.9). We set $E_{\delta 1}^{\mathcal{U}}=$ the $*$-least $\mathbb{D}_{\delta 1}$-nice witness $G$ for $R_{\delta 1}$, set $\operatorname{pred}^{\mathcal{U}}(\delta, 2), \mathbb{F}, f$ like usual, set $\mathbb{D}_{\delta 2}=f(\mathbb{F})$, and set $\xi_{\delta 2}$ to be the $\xi$ such that $R_{\delta 1}=\mathfrak{C}_{\omega}\left(S_{\xi}^{\mathbb{D}_{\delta 2}}\right)$. So either way, $\xi_{\delta 2}$ is $\mathbb{D}_{\delta 2}$-standard. After this we proceed as before.

CASE 6. Otherwise (equivalently, $u_{\delta}>0$ and $E_{\delta} \notin \mathbb{E}_{+}^{M_{\delta}}$ and $\delta$ is $\mathscr{N}$-stable and $\vartheta_{\delta}<\mathrm{OR}^{N_{\delta}}$ and $\left.\left(\vartheta_{\delta}^{+}\right)^{N_{\delta}} \leq \operatorname{lh}\left(E_{\delta}\right)\right)$

By symmetry. 
This completes the proof of part (ii) of Claim 5. In the proof of part (iv), we need substitutes $\mathbb{C}_{\alpha j}^{\prime}$ for $\mathbb{C}_{\alpha j}$ and $<_{\alpha j}^{\prime}$ for $<_{\alpha j}^{*}$ (there is, however, no $\widetilde{\mathbb{C}}_{\alpha j}$ to consider, and we do not need the notation $\mathbb{D}_{\alpha j}$ or $\left.\widetilde{\mathbb{D}}_{\alpha j}\right)$. We have the class wellorder $<W$ of $W$ (which, however, need not be a class of $W$ itself). Given $\gamma \in \mathrm{OR}^{W}$, let $\mathbb{C}^{\gamma W}$ be the $<^{W}$-least ultra-backgrounded construction of $W$ with last model $R_{\gamma}^{W}$. We only define $\xi_{\alpha 0}$ in the case that $[0, \alpha]_{\mathcal{T}}$ drops in model (but we always define $\xi_{\alpha j}$ when $j>0$ ).

Suppose $[0, \alpha]_{\mathcal{T}}$ does not drop. In this case we determine $\mathbb{C}_{\alpha 0}$ only after selecting $E_{\alpha}^{\mathcal{T}}$. We will have $Q_{\alpha 0}=L[\mathbb{E}]^{M_{\alpha 0}^{\mathcal{U}}}$ and $\pi_{\alpha 0}: M_{\alpha} \rightarrow Q_{\alpha 0}$ is elementary and $\pi_{\alpha 0} \circ i_{0 \alpha}^{\mathcal{T}}=i_{00, \alpha 0}^{\mathcal{U}}$. Let $\gamma$ be least such that $\pi_{\alpha 0}\left(\operatorname{lh}\left(E_{\alpha}^{\mathcal{T}}\right)\right)<i_{00, \alpha 0}^{\mathcal{U}}\left(\operatorname{OR}\left(R_{\gamma}\right)\right)$. Then we set $\mathbb{C}_{\alpha 0}=i_{00, \alpha 0}^{\mathcal{U}}\left(\mathbb{C}^{\gamma W}\right)$. So in $M_{\alpha 0}^{\mathcal{U}}, \mathbb{C}_{\alpha 0}$ has last model $L[\mathbb{E}] \mid \zeta$ for some $L[\mathbb{E}]$-cardinal $\zeta$, and $\pi_{\alpha 0}\left(\operatorname{lh}\left(E_{\alpha}^{\mathcal{T}}\right)\right)<\zeta$.

If $[0, \alpha]_{\mathcal{T}}$ drops or $j>0$, then $\mathbb{C}_{\alpha j}$ is defined basically as before (though it is more standard, because there is no $\widetilde{\mathbb{C}}_{\alpha j}$ etc).

Now consider $<_{\alpha j}^{*}$. Let $f=i_{00, \alpha j}^{\mathcal{U}}$. (Note that $f\left(<^{W}\right)$ need not make sense, since $<^{W}$ need not be a $W$-class.) Given $x, y \in M_{\alpha j}^{\mathcal{U}}$, set $x<_{\alpha j}^{\prime} y$ iff either (i) $\operatorname{rank}(x)<\operatorname{rank}(y)$, or (ii) $\left[\operatorname{rank}(x)=\operatorname{rank}(y)\right.$ and letting $\beta<\mathrm{OR}^{W}$ be least such that $x, y \in f\left(V_{\beta}^{W}\right)$, and letting $<_{0}$ be the $<^{W}$-least wellorder of $V_{\beta}^{W}$, then $\left.(x, y) \in f\left(<_{0}\right)\right]$. Clearly $<_{\alpha j}^{\prime}$ is a wellorder of $M_{\alpha j}^{\mathcal{U}}$; we use this in place of $<_{\alpha j}^{*}$ when selecting $E_{\alpha j}^{\mathcal{U}}$.

The rest is straightforward. This completes the proof of the theorem.

6.25 Remark. Suppose $W \models Z F C$ is an iterable premouse. Let $L[\mathbb{E}]^{W}$ be the output of the pm-ultra stack construction of $W$. Then we have the usual partial converse to the fact that $L[\mathbb{E}]^{W}$ inherits Woodins. That is, let $\delta$ be Woodin in $L[\mathbb{E}]$. Then $W \mid \delta$ is generic for the extender algebra of $L[\mathbb{E}]$ at $\delta$, and $\delta$ is Woodin in $L[\mathbb{E}][W \mid \delta]$.

The natural analogue of [5, Theorem 3.2] also holds for ultra-backgrounded constructions, and hence for the ultra-stack construction, assuming that extenders cohere the relevant iteration strategy:

6.26 Theorem. Assume ZFC and let $M$ be a countable, $k$-sound, ( $k, \mathrm{OR})$ iterable premouse, and $\Sigma$ be a $(k, \mathrm{OR})$-iteration strategy for $M$. Suppose that $i_{E}(\Sigma)=\Sigma\lceil\operatorname{Ult}(V, E$ for every short $V$-extender $E$.

Let $\mathbb{C}=\left\langle S_{\alpha}\right\rangle_{\alpha \leq \lambda}$ be an ultra-backgrounded construction. Then there is $\xi \leq \lambda$ and $\left\langle\mathcal{T}_{\alpha}\right\rangle_{\alpha \leq \xi}$ such that (i) $\mathcal{T}_{\alpha}$ is a successor length tree via $\Sigma$ with $S_{\alpha} \unlhd M_{\infty}^{\mathcal{T}_{\alpha}}$, (ii) if $\alpha<\xi$ and $S_{\alpha}=M_{\infty}^{\mathcal{T}_{\alpha}}$ then $b^{\mathcal{T}_{\alpha}}$ drops in model, (iii) if $\xi<\lambda$ then $b^{\mathcal{T}_{\xi}}$ does not drop in model.

Moreover, either:

(a) there is some ultra-backgrounded construction $\mathbb{C}$, with last model $S_{\lambda}$, such that $\mathcal{T}_{\lambda}^{\mathbb{C}}$ exists and $b^{\mathcal{T}_{\lambda}^{\mathbb{C}}}$ does not drop in model, or 
(b) the ultra-stack construction $\left\langle R_{\alpha}\right\rangle_{\alpha \in \mathrm{OR}}$ is well-defined, and there is a length OR tree $\mathcal{U}$ on $M$, via $\Sigma$, such that $R_{\mathrm{OR}}=M(\mathcal{U})$.

Proof. The proof is like that of [5, Theorem 3.2]; there are also variants of this argument in $[11, \S 5]$ and elsewhere. Let $\mathbb{C}$ be an ultra-backgrounded construction. One constructs $\mathcal{T}_{\alpha}$ by induction on $\alpha \leq \lambda$, until reaching either $\lambda$ or some appropriate $\xi<\lambda$. The induction is straightforward except for when either $S_{\alpha}$ is active or $\alpha$ is non-standard, so we consider these cases.

CASE $1 . S_{\alpha}$ is active and $\alpha$ is standard.

So $S_{\alpha}=(S, F)$ where $S=S_{\beta}$. Let $F^{*}$ be a background for $F$ (as in 6.3). Let $k: \operatorname{Ult}_{0}(S, F) \rightarrow j(S)$ be the natural factor map; so $\nu(F) \leq \operatorname{cr}(k)$. Let $j=i_{F^{*}}^{V}$ and $\kappa=\operatorname{cr}(j)$. We have $\mathcal{T}=\mathcal{T}_{\beta}$ with $S \unlhd M_{\infty}^{\mathcal{T}}$; since $\kappa$ is measurable and $M$ countable, $\operatorname{lh}(\mathcal{T}) \geq \kappa+1$. We may assume that $\operatorname{lh}(G) \leq \mathrm{OR}^{S}$ for all extenders $G$ used in $\mathcal{T}$. Then $j(S) \unlhd M_{\infty}^{j(\mathcal{T})}$, and by assumption, $j(\mathcal{T})$ is via $\Sigma$. Note $\mathcal{T} \uparrow(\kappa+1) \unlhd j(\mathcal{T})$ and $j\left([0, \kappa]_{\mathcal{T}}\right)=[0, j(\kappa)]_{j(\mathcal{T})}$, and $[0, \kappa)_{\mathcal{T}} \subseteq[0, j(\kappa)]_{j(\mathcal{T})}$, so $\kappa<_{j(\mathcal{T})} j(\kappa)$. We have $i_{\kappa, j(\kappa)}^{j(\mathcal{T})}\lceil\mathcal{P}(\kappa) \subseteq j$, by the proof of termination of comparison; in particular, $\kappa=\operatorname{cr}\left(i_{\kappa, j(\kappa)}^{j(\mathcal{T})}\right)$. Note $[0, j(\kappa)]_{j(\mathcal{T})}$ has no drops $\geq \kappa$, so $\left(\kappa^{+}\right)^{M_{\infty}^{j(\mathcal{T})}}=\left(\kappa^{+}\right)^{M_{\kappa}^{\mathcal{T}}}$. Clearly $\theta=\left(\kappa^{+}\right)^{S} \leq\left(\kappa^{+}\right)^{M_{\kappa}^{\mathcal{T}}}$ and and $S \mid \theta=M_{\infty}^{j(\mathcal{T})} \| \theta$. Let $\gamma+1=\operatorname{succ}^{j(\mathcal{T})}(\kappa, j(\kappa))$ and $E=E_{\gamma}^{j(\mathcal{T})}$. Then $\operatorname{cr}(E)=\kappa$ and $E\lceil\nu(E)$ is derived from $j$. Let $\nu=\min (\nu(E), \nu(F))$. Then

$$
E \cap\left(\left(M_{\kappa}^{\mathcal{T}} \| \theta\right) \times[\nu]^{<\omega}\right)=F\lceil\nu .
$$

SubCASE 1.1. $\left(\kappa^{+}\right)^{M_{\kappa}^{\mathcal{T}}}=\theta=\left(\kappa^{+}\right)^{S}$.

So $E, F$ are compatible; that is, $E\lceil\nu=F\lceil\nu$. By the ISC for $(S, F), \mathcal{T}$ does not use any extender of index $<\mathrm{OR}^{S}$ which is compatible with $F$.

SubSUBCASE 1.1.1. $j(S) \| \mathrm{OR}^{S}=S$.

Then $\mathcal{T}, j(\mathcal{T})$ use the same extenders with index $<\mathrm{OR}^{S}$. So by the previous paragraph, $\mathrm{OR}^{S} \leq \operatorname{lh}(E)$. If $\operatorname{lh}(E)=\mathrm{OR}^{S}$, then $E_{\gamma}^{j(\mathcal{T})}=E=F$, so $(S, F) \unlhd$ $M_{\gamma}^{j(\mathcal{T})}$, so $\mathcal{T}_{\alpha}=j(\mathcal{T}) \uparrow(\gamma+1)$ is as desired. If $\operatorname{lh}(E)>\mathrm{OR}^{S}$, then by the ISC applied to $\operatorname{ex}_{\gamma}^{j(\mathcal{T})}$, and since $S=\operatorname{ex}_{\gamma}^{j(\mathcal{T})} \| \mathrm{OR}^{S}$, we get $(S, F) \triangleleft \operatorname{ex}_{\gamma}^{j(\mathcal{T})}$, so we can set $\mathcal{T}_{\alpha}=j(\mathcal{T}) \uparrow\left(\gamma^{\prime}+1\right)$ with some $\gamma^{\prime}$.

SubsubCASE 1.1.2. $j(S) \| \mathrm{OR}^{S} \neq S$.

Then $F$ is type 1 or 3 . Let $\nu^{\prime}=\nu(F)$. By condensation arguments using $k$, $j(S) \mid \nu^{\prime}$ is active with an extender $G$ and $S=\operatorname{Ult}\left(j(S) \mid \nu^{\prime}, G\right) \| \lambda$. It follows that there is $\gamma^{\prime}$ such that $\mathcal{T} \uparrow\left(\gamma^{\prime}+1\right) \unlhd j(\mathcal{T})$ and $E_{\gamma^{\prime}}^{\mathcal{T}}=G$, but $\nu^{\prime}<\operatorname{lh}\left(E_{\gamma^{\prime}}^{j(\mathcal{T})}\right)$. Like before, $\ln (E) \geq \nu^{\prime}$, so $\ln (E)>\nu^{\prime}$ (as $\ln (G)=\nu^{\prime}$ ). So by the ISC applied to $\operatorname{ex}_{\gamma}^{j(\mathcal{T})}$, with respect to $\nu^{\prime}$, and since $\operatorname{lh}(G)=\nu^{\prime},(S, F) \unlhd M_{\gamma^{\prime}+1}^{\mathcal{T}}$, which suffices.

SuBCASE 1.2. $\left(\kappa^{+}\right)^{M_{\kappa}^{\mathcal{T}}}>\theta=\left(\kappa^{+}\right)^{S}$.

Then $\operatorname{cr}(k)=\theta$, so $F$ is type 1 . We now argue with subsubcases much as before, but using the (proofs of) [11, Theorems 4.11, 4.12, 4.15] in place of the ISC. (In [11], premice are always assumed to be below a superstrong. But the proofs adapt routinely to the superstrong setting.) 
This completes the construction of $\mathcal{T}_{\alpha}$ in this case.

CAse 2. Now suppose instead that $\alpha$ is non- $\mathbb{C}$-standard. So $\alpha=\beta+1$, and letting $\rho=\rho_{\omega}\left(S_{\alpha}\right)$, and $\mathcal{T}=\mathcal{T}_{\beta}$, we have $S_{\beta}=S_{\alpha} \|\left(\rho^{+}\right)^{S_{\alpha}} \unlhd M_{\infty}^{\mathcal{T}}$. Let $G$ be a nice witness for $S_{\alpha}$ and $j=i_{G}^{V}$. Then $S_{\alpha} \triangleleft j\left(S_{\alpha} \mid \rho\right)$, so $S_{\alpha} \triangleleft M_{\infty}^{j(\mathcal{T})}$, and since $j(\mathcal{T})$ is via $\Sigma$, this suffices to yield $\mathcal{T}_{\alpha}$.

This completes the inductive construction of the trees $\mathcal{T}_{\alpha}$.

Now suppose there is no ultra-stack construction as in part (a) of the the "moreover" clause of the theorem. Then for every ultra-backgrounded construction $\mathbb{C}$ and every $\alpha<\operatorname{lh}(\mathbb{C}), \mathcal{T}_{\alpha}^{\mathbb{C}}$ exists and if $S_{\alpha}^{\mathbb{C}}=M_{\infty}^{\mathcal{T}_{\alpha}^{\mathbb{C}}}$ then $b^{\mathcal{T}_{\alpha}^{\mathbb{C}}}$ drops in model. But then note that no ultra-backgrounded construction can break down; that is, for each $n<\omega, \mathfrak{C}_{n}\left(S_{\alpha}^{\mathbb{C}}\right)$ is $(n+1)$-universal and $\mathfrak{C}_{n+1}\left(S_{\alpha}^{\mathbb{C}}\right)$ is $(n+1)$-solid.

Let $\left\langle R_{\alpha}\right\rangle_{\alpha \in \mathrm{OR}}$ be the ultra-stack construction. We show by induction on $\alpha$ that $R_{\alpha}$ is well-defined and sound, and for each $\alpha \in \mathrm{OR}$, there is a tree $\mathcal{U}_{\alpha}$ via $\Sigma$ such that $R_{\alpha} \triangleleft M_{\infty}^{\mathcal{U}_{\alpha}}$, and by taking $\mathcal{U}_{\alpha}$ of minimal length, then $\mathcal{U}_{\alpha} \unlhd \mathcal{U}_{\beta}$ for $\alpha<\beta$. This suffices, because then $\mathcal{U}=\bigcup_{\alpha \in \mathrm{OR}} \mathcal{U}_{\alpha}$ is as desired.

So suppose $R_{\alpha}$ is defined and we have $\mathcal{U}_{\alpha}$ with $R_{\alpha} \unlhd M_{\infty}^{\mathcal{U}_{\alpha}}$. Let $\mathbb{C}$ be an ultra-backgrounded construction with $S_{\lambda}^{\mathbb{C}}=R_{\alpha}$.

Now $R_{\alpha} \triangleleft M_{\infty}^{\mathcal{U}_{\alpha}}$. For suppose $R_{\alpha}=M_{\infty}^{\mathcal{U}_{\alpha}}$. Then because (a) fails, $b^{\mathcal{U}_{\alpha}}$ drops in model, so $R_{\alpha}$ is not sound. Let $R=\mathfrak{C}_{\omega}\left(M_{\infty}^{\mathcal{U}_{\alpha}}\right)$ (this exists and in fact $R \triangleleft M_{\xi}^{\mathcal{U}_{\alpha}}$ for some $\xi$ ). So $R \not R_{\alpha}$. But $R_{\alpha}=S_{\lambda}^{\mathbb{C}}$ is produced by ultra-backgrounded construction, so $R$ is also, so by maximality of the ultra-stack construction, $R \unlhd R_{\alpha}$, a contradiction.

So $R_{\alpha} \triangleleft M_{\infty}^{\mathcal{U}_{\alpha}}$. Now consider the sound premice $R$ which project to $\mathrm{OR}^{R_{\alpha}}$ and form the stack $R_{\alpha+1}$ above $R_{\alpha}$. These $R$ are produced by ultra-backgrounded construction, and $R_{\alpha} \triangleleft R$, so we get $\mathcal{T}_{R}$ such that $R \unlhd M_{\infty}^{\mathcal{T}_{R}}$, and note $\mathcal{U}_{\alpha} \triangleleft \mathcal{T}_{R}$, and it easily follows that $R \unlhd M_{\infty}^{\mathcal{U}_{\alpha}}$, giving well-definedness of $R_{\alpha+1}$. And note we get either $\mathcal{U}_{\alpha+1}=\mathcal{U}_{\alpha}$, or $\mathcal{U}_{\alpha+1}=\mathcal{U}_{\alpha}{ }^{\curlywedge}\langle E\rangle$ where $\operatorname{lh}(E)=\operatorname{OR}\left(R_{\alpha+1}\right)$.

Limit stages are easy. This completes the proof.

\section{Questions}

Since condensation follows from solidity and normal iterability, we ask:

- Let $m<\omega$ and let $M$ be an $m$-sound, $\left(m, \omega_{1}+1\right)$-iterable premouse. Is $M(m+1)$-universal? Is $M(m+1)$-solid?

- Let $M$ be a 1 -sound $\left(0, \omega_{1}+1\right)$-iterable premouse. Is $M$ Dodd-solid?

We conjecture that the answer in each case is "yes", ${ }^{42}$ at least if $M$ has no superstrong initial segments. However, it appears less clear how to prove these things than it is condensation; if one attempts an approach similar to the proof of condensation (from normal iterability) then, at least naïvely, structures arise

\footnotetext{
${ }^{42}$ The author has since confirmed this conjecture, including superstrongs; see [7].
} 
similar to bicephali $B$, but the premice involved may fail to be $\rho(B)$-sound. Such generalizations of cephalanxes also arise. This lack of soundness makes the analysis of these structures less clear than those considered in this paper.

One also uses $\left(0, \omega_{1}, \omega_{1}+1\right)^{*}$-iterability of pseudo-premice to prove that they satisfy the ISC. It seems that one might get around this by avoiding pseudopremice entirely (in the proof of 6.18), using bicephali and cephalanxes instead. Extra difficulties also seem to arise here with superstrong premice. ${ }^{43}$

\section{References}

[1] S. Jackson, R. Ketchersid, F. Schlutzenberg, and W. H. Woodin. Determinacy and jónsson cardinals in $L(\mathbb{R})$. The Journal of Symbolic Logic, 79(4):1184-1198, 2014.

[2] Ronald Jensen, Ernest Schimmerling, Ralf Schindler, and John Steel. Stacking mice. The Journal of Symbolic Logic, 74(1):315-335, 2009.

[3] William J. Mitchell and John R. Steel. Fine structure and iteration trees, volume 3 of Lecture Notes in Logic. Springer-Verlag, Berlin, 1994.

[4] E. Schimmerling and J. R. Steel. Fine structure for tame inner models. J. Symbolic Logic, 61(2):621-639, 1996.

[5] E. Schimmerling and J. R. Steel. The maximality of the core model. Trans. Amer. Math. Soc., 351(8):3119-3141, 1999.

[6] Ralf Schindler. Notes on a talk of Farmer Schlutzenberg: Fine structure from normal iterability. Available at https://ivv5hpp.unimuenster.de/u/rds/muenster_meeting_2015.html, 2015.

[7] F. Schlutzenberg. Fine structure from normal iterability. In preparation.

[8] F. Schlutzenberg and N. Trang. The fine structure of operator mice. To appear. ArXiv:1604.00083.

[9] Farmer Schlutzenberg. Reconstructing copying and condensation. To appear. Available at https://sites.google.com/site/schlutzenberg/home$1 /$ research/papers-and-preprints.

[10] Farmer Schlutzenberg. Reconstructing resurrection. Unpublished notes. Arxiv: 1811.04236 .

[11] Farmer Schlutzenberg. Measures in mice. PhD thesis, University of California, Berkeley, 2007. Arxiv: 1301.4702.

\footnotetext{
${ }^{43}$ Actually, there is an easy direct proof that normal iterability suffices for this result, which does not need any bicephalus-style comparisons. This will also appear in [7].
} 
[12] Farmer Schlutzenberg. The definability of $\mathbb{E}$ in self-iterable mice. To appear. ArXiv: 1412.0085, 2014.

[13] Farmer Schlutzenberg. The definability of the extender sequence $\mathbb{E}$ from $\mathbb{E}\left\lceil\aleph_{1}\right.$ in $L[\mathbb{E}] .2019$. ArXiv: 1906.00276.

[14] John Steel. Core models with more Woodin cardinals. 67(3):1197-1226, 2002 .

[15] John R. Steel. Local $K^{c}$ constructions. The Journal of Symbolic Logic, 72(3):721-737, 2007.

[16] John R. Steel. An outline of inner model theory. In Handbook of set theory. Vols. 1, 2, 3, pages 1595-1684. Springer, Dordrecht, 2010.

[17] Hugh Woodin. The fine structure of suitable extender models I. 2014.

[18] Martin Zeman. Inner models and large cardinals, volume 5 of de Gruyter Series in Logic and its Applications. Walter de Gruyter \& Co., Berlin, 2002.

[19] Martin Zeman. Dodd parameters and $\lambda$-indexing of extenders. J. Math. Log., 4(1):73-108, 2004. 